\title{
Air-Ground Integration Experiment
}

Karen DiMeo

Randy Sollenberger, Ph.D.

Parimal Kopardekar, Ph.D.

Sandy Lozito

Margaret-Anne Mackintosh

Kim Cardosi, Ph.D.

Tom McCloy, Ph.D.

January 2002

DOT/FAA/CT-TN02/06

Document is available to the public through the National Technical Information Service, Springfield, Virginia 22161

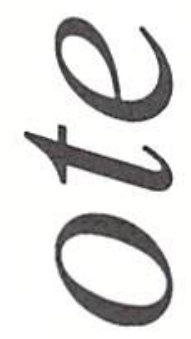

U.S. Department of Transportation Federal Aviation Administration

William J. Hughes Technical Center

Atlantic City International Airport, NJ 08405 
This document is disseminated under the sponsorship of the U.S. Department of Transportation in the interest of information exchange. The United States Government assumes no liability for the contents or use thereof. The United States Government does not endorse products or manufacturers. Trade or manufacturer's names appear herein solely because they are considered essential to the objective of this report. This document does not constitute FAA certification policy. 
Technical Report Documentation Page

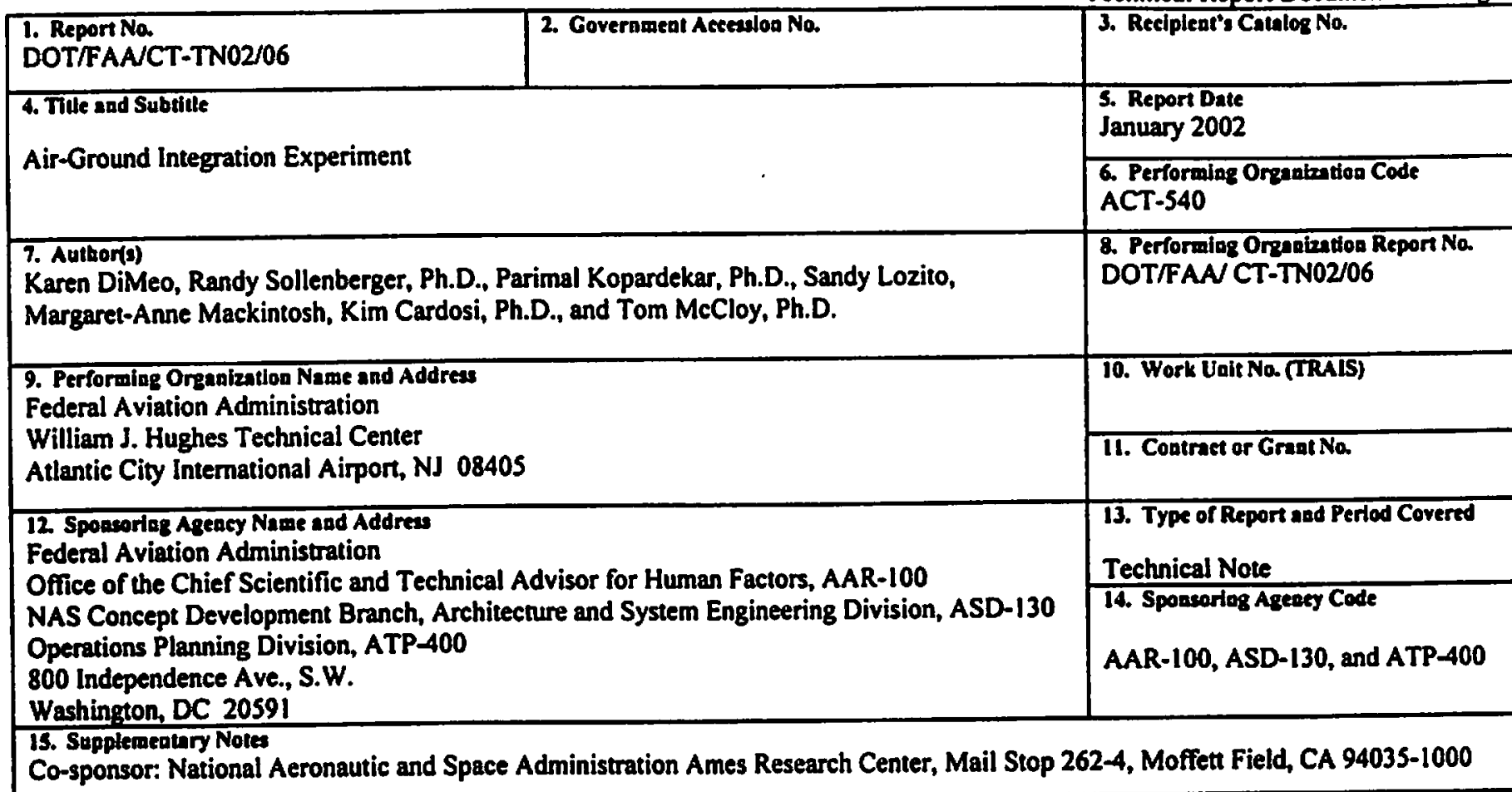

\section{Abetract}

The concept of free flight is intended to provide increased flexibility and efficiency throughout the global airspace system. This idea could potentially shif aireraft separation responsibility from air traffic controllers to flight crews creating a 'shared-separation' authority environment. A real-time, human-in-the-loop study was conducted using facilities at NASA Ames Research Center and the FAA William J. Hughes Technical Center. The goal was to collect data from controllers and pilots on shared-separation procedures, information requirements, workload, and situation awareness. The experiment consisted of four conditions that varied levels of controller and flight crew separation responsibifities. Twelve controllers and six pilots were provided with enhanced traffic and conflict alerting systems. Results indicated that while safety was not compromised, pilots and controllers had differing opinions regarding the application of these new tools and the feasibility of the operational concept. This limited investigation demonstrated the need to further explore the shared-separation concept. 
$\therefore \because \because \div ? \div$

$$
\begin{aligned}
& \therefore \quad \therefore \\
& \text {. . . . }
\end{aligned}
$$

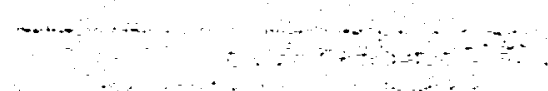
$\therefore \quad \therefore \quad \therefore=$

$\because \cdots \quad \because \cdots$

$\begin{array}{ccc}\cdots & \ddots & \cdots \\ \cdots & \ddots & \cdots\end{array}$

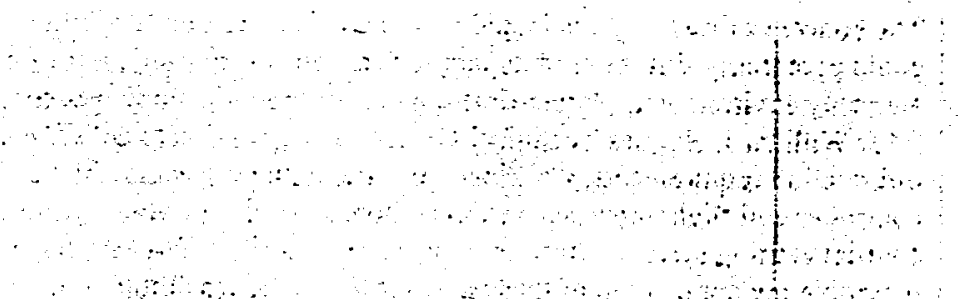

$\therefore \therefore$ 


\section{ACKNOWLEDGMENTS}

The authors wish to acknowledge several people who contributed their expert talent and many long hours to this study. Without their hard work and outstanding support, this study would not have been a success.

Air Traffic Control Subject Matter Experts: Philip Bassett (ZJX), Alan Yost (Volpe Center), Alice Hardison (FAA, ACT-510), and Tim Holth (Titan Systems).

Study Design, Execution, and Analysis Support at the NASA Ames Research Center (ARC): Alison McGann, Melisa Dunbar, Patricia Cashion, Dave Jara, and Paul Picciano (all with the San Jose State University Foundation), and Tom Kozon (Raytheon, Inc.).

Study Design, Execution, and Analysis Support at the FAA William J. Hughes Technical Center (WJHTC): Nicole Sacco, Kevin Carmen, and Fatiha Jackson (Titan Systems), and Marianne Abbonizio (FAA, ACT-540/ Embry Riddle Aeronautical University).

Facility and Laboratory Support at the FAA WJHTC: Integration and Interoperability Facility staff, Research and Development Human Factors Laboratory staff, Pseudo Aircraft Simulation Laboratory staff, and Target Generation Facility staff.

Facility and Laboratory Support at the NASA ARC: Crew Vehicle Systems Research Facility staff.

Memphis ARTCC: National Air Traffic Controllers Association, study participant controllers, supervisors, and facility managers. 


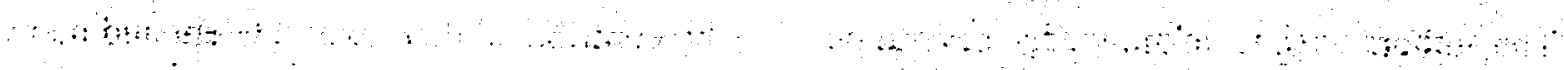

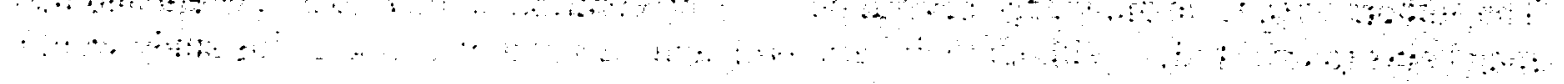

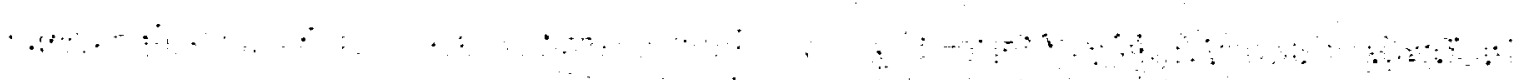

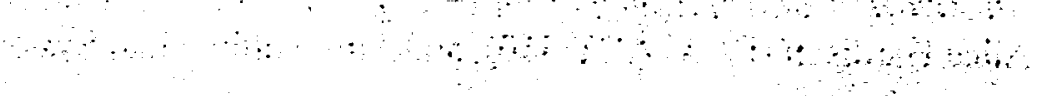

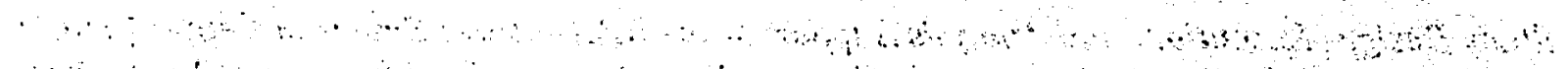

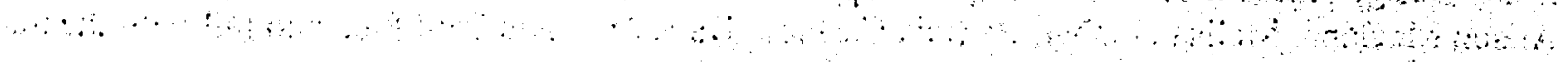

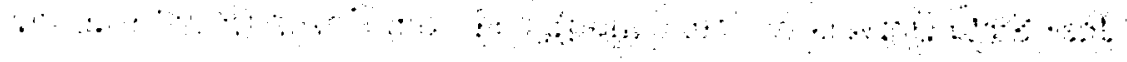

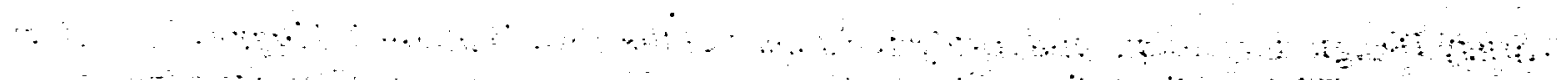

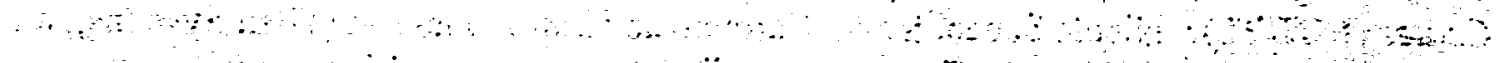

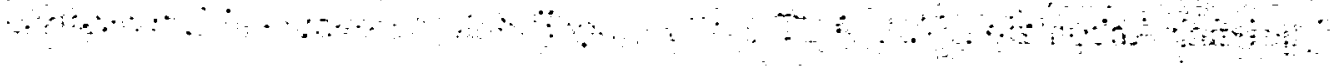

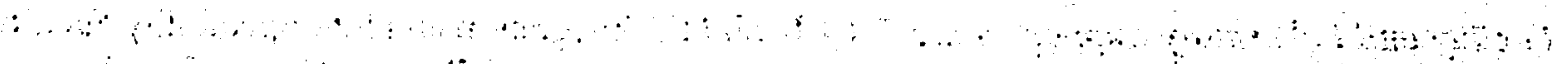

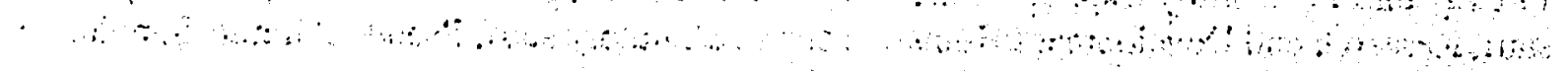

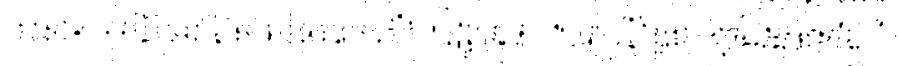

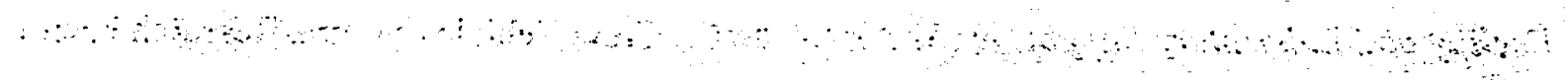

$$
\begin{aligned}
& \because+\cdots
\end{aligned}
$$

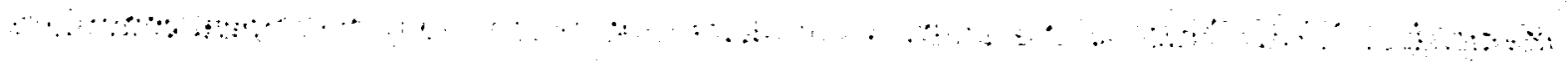

$$
\begin{aligned}
& \therefore-\cdots+\cdots, \cdots
\end{aligned}
$$

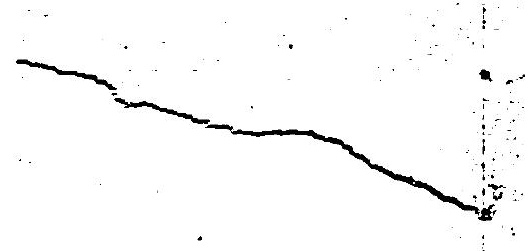


ACKNOWLEDGMENTS iii

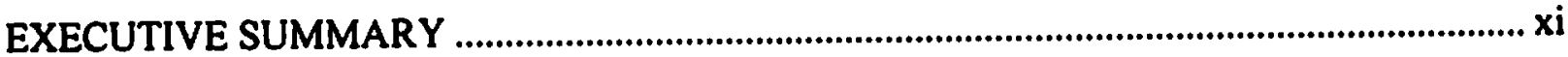

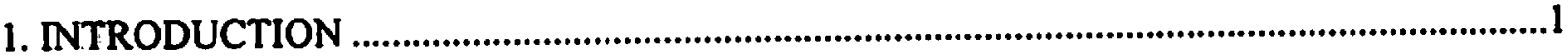

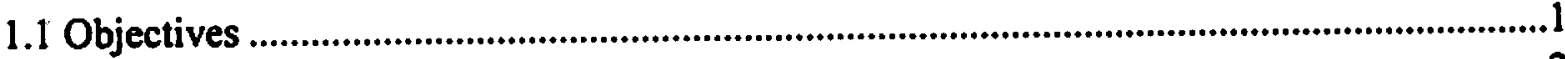

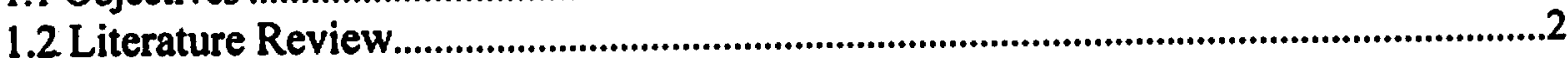

2. METHOD

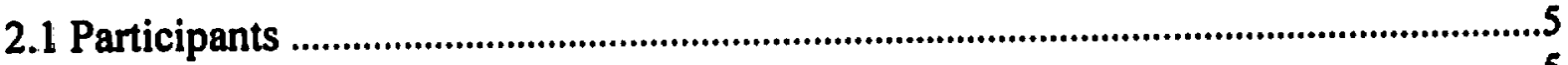

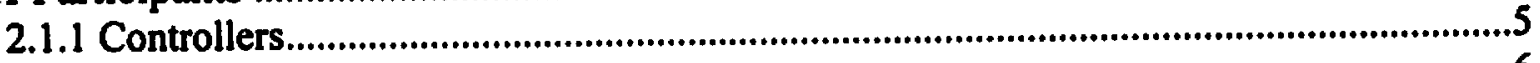

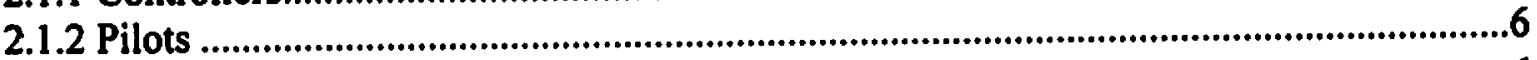

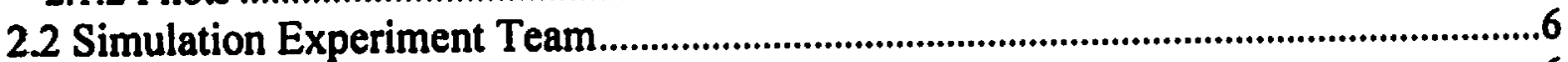

2.2.1 Expert Observers.....................................................................................................6

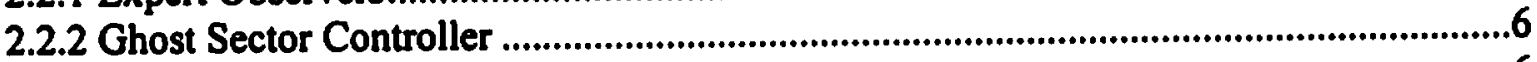

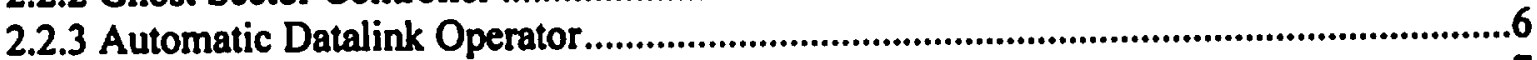

2.2.4 WJHTC Simulation Pilots and Laboratory Coordinators ..............................................7

2.2.5 Intruder Simulation Pilot...........................................................................................

2.3 Facilities and Equipment Overview ............................................................................

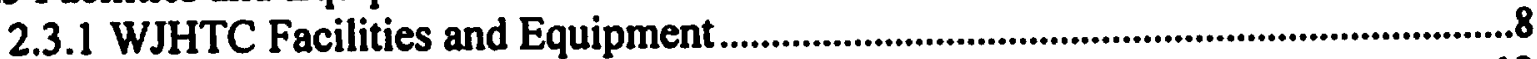

2.3.2 NASA Crew-Vehicle Systems Research Facility and ARC Simulator ........................12

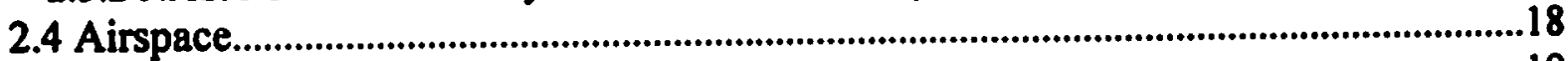

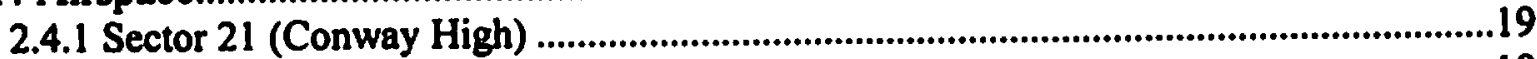

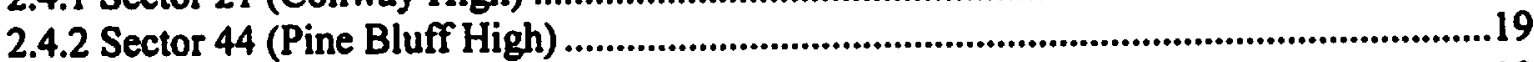

2.5 Experimental Conditions ......................................................................................19

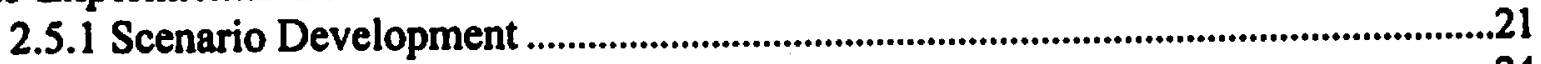

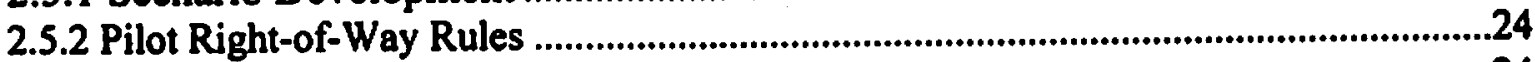

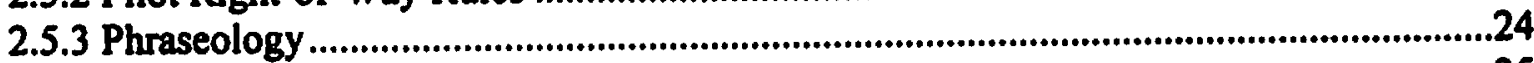

2.5.4 Frequencies ........................................................................................................25

2.6 Simulation Constraints and Assumptions ................................................................26

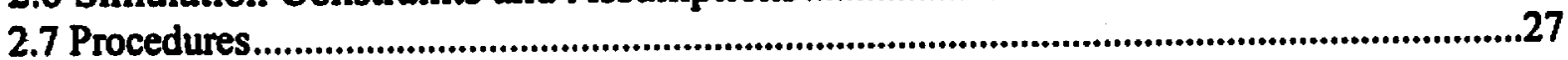

2.7.1 WJHTC Pre-Simulation Activities ...............................................................27

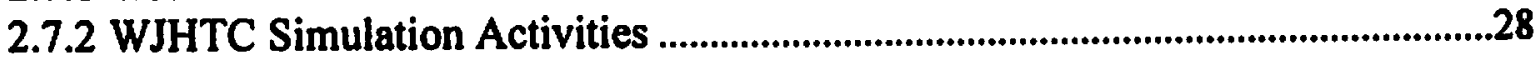

2.7.3 NASA ARC Pre-Simulation Activities...........................................................29

2.7.4 NASA ARC Simulation Activities ..................................................................30

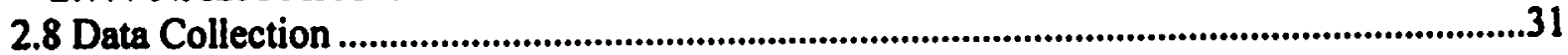

2.8.1 Ground-Side Subjective Data .............................................................................31

2.8.2 Ground-Side Objective Data ............................................................................35

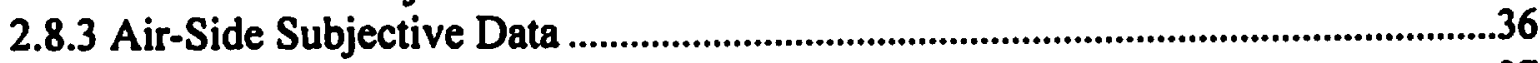

2.8.4 Air-Side Objective Data ...................................................................................37 
Table of Contents (Cont.)

Page

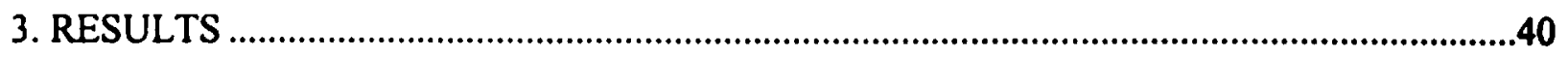

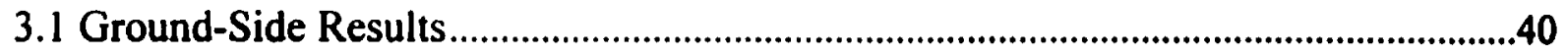

3.1.1 Operational Issues that Affect Shared-Separation Operations.........................................40

3.1.2 Information Requirements and Procedures.........................................................................45

3.1.3 Controller Workload and Situation Awareness ........................................................49

3.1.4 Exit Form Responses and Ratings ................................................................................55

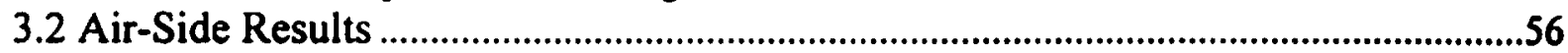

3.2.1 Operational Issues that Affect Shared-Separation Operations...........................................56

3.2.2 Information Requirements and Procedures.........................................................................61

3.2.3 Pilot Workload and Situation Awareness ....................................................................65

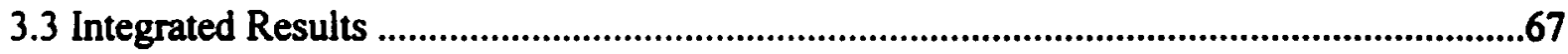

3.3.1 Operational Issues that Affect Shared-Separation Operations............................................67

3.3.2 Information Requirements and Procedures.........................................................................74

3.3.3 Controller and Pilot Workload and Situation Awareness ..............................................76

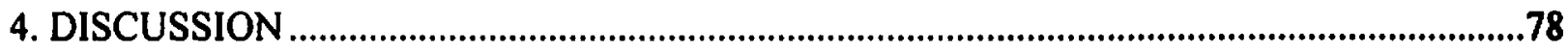

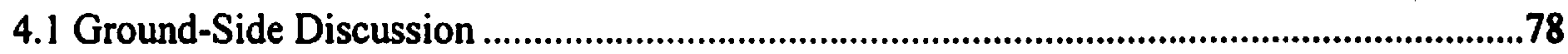

4.1.1 Operational Issues that Affect Shared-Separation Operations.........................................78

4.1.2 Controller Information Requirements and Procedures .....................................................79

4.1.3 Controller Workload and Situation Awareness .............................................................80

4.2 Air-Side Discussion..........................................................................................................82

4.2.1 Operational Issues that Affect Shared-Separation Operations...........................................82

4.2.2 Flight Crew Information Requirements and Procedures.................................................83

4.2.3 Flight Crew Workload and Situation Awareness............................................................84

4.3 Integrated Discussion ........................................................................................................85

4.3.1 Operational Issues that Affect Shared-Separation Operations..........................................85

4.3.2 Information Requirements and Procedures......................................................................89

4.3.3 Workload and Situation Awareness..........................................................................90

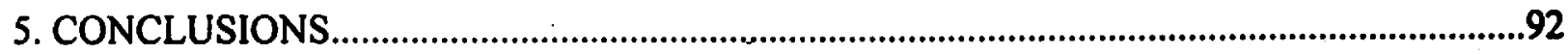

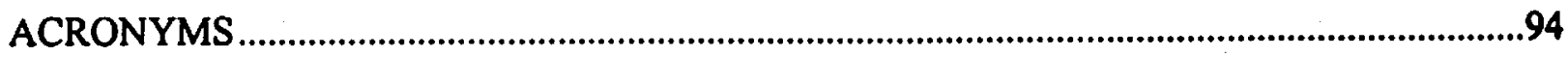

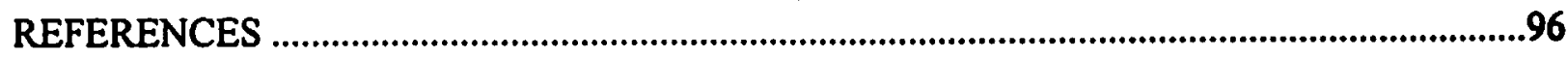

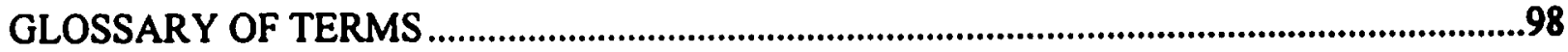


Appendices

A - Controller Daily Schedule

B - Controller Briefing

C - Controller and Expert Observer Forms

D - Human Research Minimal Risk Consent

E - Pilot Daily Schedule

F - Pilot Forms

G - ANOVA Results

List of lllustrations

Figure

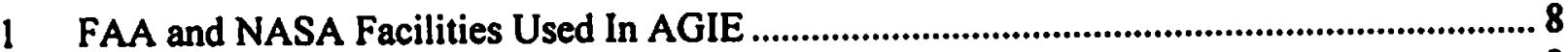

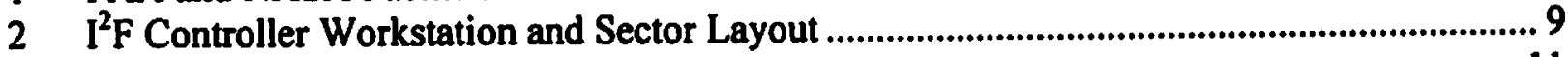

3 Workload Assessment Keypad ........................................................................................ 11

4 NASA ARC Simulator Flight Deck Layout .................................................................... 13

5 Relationship Of New Airborne Alerting Logic To TCAS Logic....................................... 14

6 CDTI-AL Depicting Non-Threat Aircraft .................................................................. 15

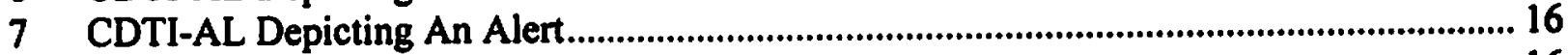

8 Control Box For Pilot Selectable Features...................................................................... 16

9 CDTI-AL Depicting Predictors Selected ............................................................................ 17

10 ZME Sectors 21 and 44.............................................................................................. 18

11 Controller Mean Ratings For Time Available For Separation And Coordination................ 41

12 Controller Mean Ratings Of Level Of Safety For Procedures............................................. 41

13 Mean Frequency of URET Conflict Alerts .......................................................................42 42

14 Mean Duration Of URET Red And Yellow Conflict Alerts................................................ 43

15 Controller Mean Ratings Of Information To Resolve Conflicts.......................................... 45

16 Controller Mean Ratings For URET Conflict Alert Timeliness .......................................... 46

17 Controller Mean Ratings For Usefulness Of Air $\leftrightarrow$ Air Communications ........................... 47

18 Controller Mean Ratings For The Helpfulness Of Shared-Separation ................................. 48

19 Mean Frequency Of URET Trial Plans............................................................................... 48

20 Controller Mean Workload Ratings: Physical, Mental, And Overall ................................... 49

21 Controller Mean Workload Ratings: Specific Measures ...................................................... 50

22 Controller Mean Ratings For Feeling Rushed And Bored.................................................. 50

23 Controller Mean Ratings Of Overall Situation Awareness.................................................. 51

24 R-Side Controller Mean Ratings For Interval Workload................................................... 52

25 D-Side Controller Mean Ratings For Interval Workload................................................... 53

26 Expert Observer Mean Ratings Of Controller Physical Taskload .......................................... 54

27 Mean Frequency Of Ground $\rightarrow$ Air And Land Line Ptts ...................................................... 54

28 Mean Duration Per Transmission Of Ground $\rightarrow$ Air And Land Line Ptts ............................. 55

29 Mean Percentage Of Time Pilot Participants Spent At Each Map Range Level ................... 64

30 Controller And Pilot Participant Mean Ratings Of Safety....................................................6 68

31 Mean Frequency Of Air $\leftrightarrow$ Ground Transactions ............................................................. 73

32 Mean Duration Of Air $\leftrightarrow$ Ground Transactions ............................................................. 74

33 Controller And Pilot Participant Mean Ratings For

Time Available To Assure Safe Separation 
34 Controller And Pilot Participant Mean Ratings For Time For Coordination And

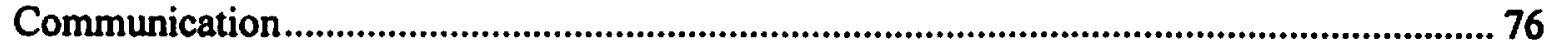

35 Controller And Pilot Participant Mean Workload Ratings ...................................................... 77

36 Controller And Pilot Participant Mean Ratings For Overall Situation Awareness................ 78

Table

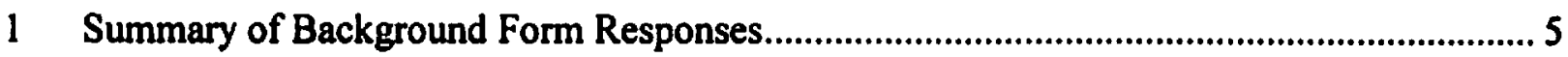

2 Experimental Condition Characteristic Summary …............................................................. 22

3 Traffic Scenario Characteristics............................................................................................ 23

4 Additional Controller Simulation Phraseology ..................................................................... 25

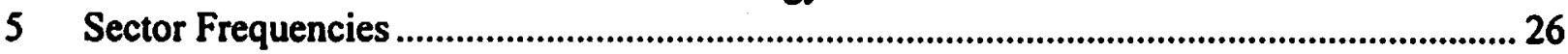

6 Run Order by Group ................................................................................................................ 29

7 Ground-Side Subjective Data .............................................................................................. 34

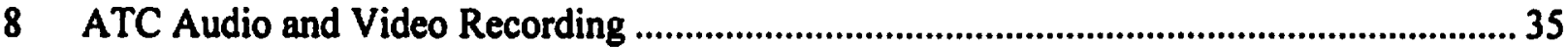

9 Ground-Side Objective Data Summary .................................................................................... 36

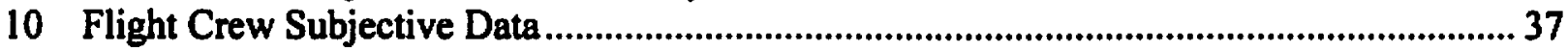

11 Air-Side Audio and Video Recordings ................................................................................ 38

12 Air-Side Objective Data Summary ............................................................................................ 39

13 Descriptive Statistics for Altitude-Resolved Planned Conflicts Involving WJHTC Simulation Pilots ....................................................................................... 44

14 Descriptive Statistics for Controller Vector-Resolved Planned Conflicts

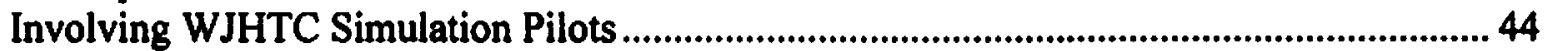

15 Controller Frequencies of Air $\leftrightarrow$ Air Communication Monitoring............................................ 46

16 Controller Role and Separation Responsibility Confusion ......................................................... 55

17 Controller Mean Ratings for Simulation Realism and Training ................................................ 56

18 Pilot Participant AHP Preference Ratings for Flight Safety ................................................. 57

19 Pilot Participant Mean Intruder-Detection Times....................................................................58 58

20 Pilot Participant Mean Ratings of the Ease of Detecting Conflicts Prior to Alert or Controller Advisory................................................................................5 58

21 Pilot Participant Mean Ratings of the CDTI-AL Effectiveness for Shared-Separation ....... 59

22 Pilot Participant Preference for Flight-Efficiency ..................................................................... 59

23 Pilot Participant Mean Ratings for the Amount of Time

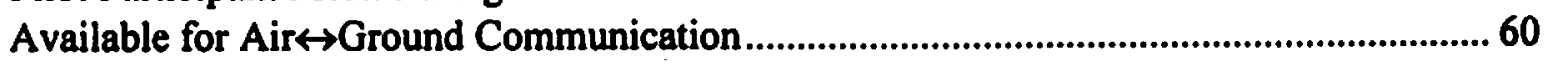

24 Pilot Participant Mean Ratings Related to Air $\leftrightarrow$ Air Communication ....................................61

25 Pilot Participant Mean Ratings of Shared-Separation ............................................................6.6. 62

26 Pilot Participant Mean Ratings of Separation Responsibility Confusion ..............................6.6.

27 Pilot Participant Mean Ratings of Information ........................................................................6 63

28 Pilot Participant Mean Ratings of the Timeliness of CDTI-AL Alerts ..................................63 63

29 Mean Percentage of Time Pilot Participants Spent Monitoring the CDTI-AL......................63

30 Pilot Participant Preference for Reducing Workload................................................................65 65

31 Pilot Participant Mean Workload Ratings ..............................................................................6 66

32 Pilot Participant Preference for Maintaining Situation Awareness .......................................67

33 Aircraft Maneuvering Mean Start Times During Shared-Separation .....................................69

34 Frequency of CDTI-AL Alerts in Relation to Maneuver Start Times ...................................69

35 Frequency and Type of Maneuvers Issued by Controllers to Resolve Conflicts Between the Pilot Participants and the Intruder Simulation Pilot.......................................... 70 
36 Frequency and Type of Maneuvers Initiated by Pilot Participants and/or Intruder Simulation Pilot to Resolve Conflicts..

37 Maneuvers Used by Controllers and/or Pilot Participants to Resolve Conflicts Involving the NASA ARC Simulator

38 Descriptive Statistics for Minimum Horizontal Distance for Conflicts Involving NASA ARC Simulator

39 Summary of Integrated Results... 
?

2 


\section{EXECUTIVE SUMMARY}

One idea of the free flight concept suggests shifting aircraft separation responsibility from air traffic controllers to flight crews. This creates a 'shared-separation' authority environment. Potential benefits of shared-separation in free flight include improved safety through enhanced conflict detection and resolution capabilities, more flexibility to manage flight operations, and better decision-making tools for air traffic controllers and flight crews. The Federal Aviation Administration (FAA), National Aeronautics and Space Administration (NASA), and the Volpe National Transportation Systems Center completed the first integrated, high fidelity, real-time, human-in-the-loop simulation study of this concept in February 2000. The FAA (AAR-100, ASD-130, and ATP-400) and NASA Ames Research Center (ARC) (Advanced Air Transportation Technologies Program) co-sponsored the study, termed Air-Ground Integration Experiment (AGIE).

AGIE provided an initial examination of the effect of shared-separation authority on flight operations when both air and ground have enhanced traffic and conflict alerting systems. The NASA ARC developed the Cockpit Display of Traffic Information with Alerting Logic (CDTI-AL) prototype, which served as the decision support tool for the flight crews. The MITRE Corporation-developed User Request Evaluation Tool (URET) was available to air traffic controllers. The objectives of the study were: to identify operational issues (e.g., communications, procedures) that affect shared-separation operations, to provide recommendations for the information requirements and procedures necessary to facilitate sharedseparation operations, and to evaluate the effect of shifting separation authority on controller and pilot workload and situation awareness.

AGIE was conducted concurrently using simulation facilities located at the FAA William J. Hughes Technical Center on the east coast and NASA ARC on the west coast. The simulation, conducted over a 4-week period, included six pilot participants, 12 certified professional controllers, and four operations supervisors as study participants. Expert observers (EO), who were subject matter experts, observed the simulation and recorded interesting observations.

Two Memphis Air Route Traffic Control Center (ARTCC) sectors, sectors 21 and 44, were emulated in the experiment. All adjacent surrounding sectors were combined on a single position, collectively called sector 78 , which was staffed by a member of the experiment team. The simulation consisted of four conditions defined by various levels of controller and flight crew shared-separation responsibilities. The conditions were Current Operations $(\mathrm{CO}), \mathrm{CO}$ with CDTI-AL (CO:CDTI), Shared-Separation Level 1 (SS:L1), and Shared-Separation Level 2 (SS:L2). Each condition used a different set of procedures that reflected changing roles and responsibilities for the participants. Current standard separation rules of $5 \mathrm{~nm}$ horizontal or $1000 / 2000 \mathrm{ft}$ vertical (as appropriate) were observed for all conditions. All flight crew and controller participants were exposed to each condition, creating a within-subjects design.

Scenarios were developed from flight plans extracted from Memphis ARTCC System Analysis and Recording tapes and accompanying Adaptation Control Environment System configuration tapes obtained from the field. The data allowed for the realistic representation of sector 
boundaries, jet routes, and fixes for the simulated sectors. Each of the four data collection runs (CO, CO:CDTI, SS:L1, and SS:L2) had 16 planned conflicts, 8 in each sector, involving two aircraft converging at acute angles.

Subjective and objective data were collected from participant controllers and pilots, the air traffic control (ATC) environment, and the flight deck. The ground-side (controller and ATC) objective data included communications, separation errors, URET alerts and trial plans, minimum separation distance (MSD), traffic density, the number of free flight cancellations, and other data. The air-side (pilots and flight deck) objective data consisted of communications, separation errors, CDTI-AL alerts, MSD, the number of free flight cancellations, and other data. Both the ground-side and air-side subjective data consisted of workload, situation awareness ratings, experiences with shared-separation, traffic realism, and other details using post-run and exit forms. EOs also recorded some critical observations such as free flight cancellations.

In general, the participant controllers had concerns regarding the feasibility of shared-separation conditions as simulated in this study. Controllers reported higher workload and expressed safety concerns under shared-separation conditions, which was demonstrated by their free flight cancellations. Controllers also preferred to resolve conflicts earlier than pilots and tended to cancel free flight when they perceived pilots were delaying the conflict resolution. However, their level of situation awareness was high across all conditions. The pilot participants preferred shared-separation conditions, particularly the condition in which they had the highest level of separation responsibility (SS:L2). They rated both shared-separation conditions as being relatively safer than current operations and as providing more situation awareness. Although it is premature to identify the best possible shared-separation level, the results of this study demonstrate the need to conduct further research in this area. 


\section{INTRODUCTION}

One element of the free flight concept, as described by the RTCA Task Force 3 (1995), suggests placing more responsibility on flight crews to maintain safe separation from other aircraft in the National Airspace System (NAS). This idea could potentially shift aircraft separation responsibility from air traffic controllers to flight crews creating a 'shared-separation' authority environment. The guiding principle of the free flight concept is to provide benefits to users and service providers. Some of the possible benefits include improved safety through enhanced conflict detection and resolution capabilities, more flexibility to manage flight operations, and better decision-making tools for air traffic controllers and flight crews. To exercise these benefits, there may be a need to supply traffic information to flight crews, and develop operating methods and tools for both the air and ground to assure safety. The Air Traffic Services Concept of Operations 2005 (FAA, 1998) promotes similar free flight ideas for shared-separation responsibility that also include trajectory negotiation between the users and air traffic controllers, user collaboration with controllers to determine optimal schedules and trajectories, and training and procedures. In addition, recent work on Distributed Air-Ground Traffic Management at the National Aeronautics and Space Administration (NASA) (NASA, 1999) reflects some of these same considerations for this new concept of operations.

To investigate some of these concepts, the NASA Ames Research Center (ARC) has developed a Cockpit Display of Traffic Information (CDTI) prototype. The CDTI includes embedded conflict-alerting logic that predicts the probability of an encounter with another aircraft. The CDTI with alerting logic (CDTI-AL) assumes Automatic Dependent Surveillance-Broadcast (ADS-B) technology to supply the position and trajectory information of all proximal air traffic. This prototype 'decision support tool' is intended to enhance flight crew situation awareness and provide more autonomy in the NAS. In addition, a ground-based conflict probe and trialplanning tool has been developed for use by air traffic controllers. This prototype decision support tool, entitled User Request and Evaluation Tool (URET), is currently fielded for daily use at the Indianapolis and Memphis Air Route Traffic Control Centers (ARTCCs) and is a key component of the Federal Aviation Administration (FAA) Free Flight Phase I Program. There have been studies done on each of these tools individually, but there is a need to investigate how they might impact procedures and human performance in a shared-separation environment.

The FAA, NASA, and the Volpe National Transportation Systems Center (VNTSC) have begun a collaborative research effort to explore some of these free flight issues. The first integrated, high fidelity, real-time, human-in-the-loop simulation of a planned series of studies began in Fall 1999 and was completed in February 2000. The concept exploration study termed the AirGround Integration Experiment (AGIE) was co-sponsored by the FAA (AAR-100, ASD-130, ATP-400) and the NASA Advanced Air Transportation Technologies Project.

\subsection{Objectives}

This experiment provided an initial examination of the effect of shared-separation authority on flight operations when both air and ground operators have enhanced traffic and conflict alerting systems. There was a strong emphasis on identifying and evaluating human factors issues. The specific objectives were 
- to identify operational issues (e.g., communications and procedures) that affect sharedseparation operations,

- to provide recommendations for the information requirements and procedures necessary to facilitate shared-separation operations, and

- to evaluate the effect of shifting separation authority on controller and pilot workload and situation awareness.

\subsection{Literature Review}

Several studies have investigated various aspects of the implementation of free flight. Many of these studies have focused on the tools that pilots and controllers will require to allow for increased flexibility in routing and separation responsibility. For example, Pekela and Hilburn (1998) conducted a free flight study in which military controllers were given a plan view display (PVD) and a prototype CDTI. Although the CDTI would typically reside on the flight deck, this study provided a CDTI view to the controller for experimental purposes. The focus of the experiment was to examine workload, visual scanning, and monitoring performance. The CDTI displayed two views on a split screen, one horizontal (plan view), and one vertical (altitude elevation view). The CDTI display incorporated conflict detection and resolution, which provided a view similar to that of the cockpit view. All of the controllers strongly agreed that the CDTI was a useful tool. Controllers tended to rely more on the vertical view compared to the horizontal. However, under high traffic conditions, the controllers tended to revert to the PVD. The controllers relied more on the CDTI during periods of conflict and more on the PVD during normal operations. Some of the controllers increased reliance on the CDTI as they became more familiar with its operation. In terms of conflict detection performance, there were no clear advantages between the PVD and CDTI displays. In terms of resolution detection time, there was a slight advantage to the use of the CDTI over the PVD. Pekela and Hilburn suggested that there is a need to redesign air traffic control (ATC) displays in order to accommodate additional dimensional approaches to conflict resolution, which may occur more frequently in free flight situations. They also suggested a look-ahead time of a little more than 5 minutes for conflict probes.

Kerns (1999) conducted a study on the usefulness of URET in helping controllers manage traffic in an unstructured environment. Controllers judged URET to have a favorable impact on safety and ATC performance, and these benefits were judged most pronounced in the free flight condition. Endsley, Sollenberger, Nakata, and Stein (2000) also reported enhanced displays may provide help for controllers under free flight conditions. In this study, the simulated enhanced display contained text information about transitioning aircraft on an ATC radar display. The researchers recommended further exploration of the concept of enhanced displays with an effort to integrate the additional display information with the controller radar picture.

Other studies have focused on the effects that shared-separation responsibility may have on controller workload, situation awareness, and perceived safety. Endsley, Mogford, Allendoerfer, Snyder, and Stein (1997) and Endsley (1997) reported that controllers acting as passive monitors during free flight may show a decrease in situation awareness, might show an increase in workload due to different responsibilities, and have problems making timely interventions. Their 
work also indicated that communications requirements may significantly increase under free flight conditions due to the need for controllers to accurately obtain pilot intent information, and to provide additional information.

Hilburn, Bakker, and Pekela (1998) reported the importance of giving controllers information on aircraft intent during free flight situations. There were no more errors in determining possible separation violations between the conditions of intent notification and without intent notification, but controllers reported more possible conflicts under free flight with no intent, compared to free flight with intent scenarios. Some controllers felt that sharing intent information would increase safety. Hilburn et al. also reported that controller subjective and objective workload could be reduced using free flight compared to conventional controlled flight. Under low-density traffic conditions, workload was reduced more for free flight with intent scenarios compared to free flight without intent scenarios. Under high traffic conditions, there was no apparent reduction in controller workload because of shared intent information. In addition, several controllers in this study expressed concern with reliance on automated conflict detection tools.

In a study conducted by Corker, Fleming, and Lane (2001), controllers managed traffic under varying levels of separation authority. Different mixes of free flight equipage were manipulated to examine the potential effects of equipage upon distribution of separation authority. Their findings indicated that controllers were more likely to take direct control of aircraft in sharedseparation scenarios with the addition of more aircraft maintaining their own separation. The study also revealed that when a majority of the aircraft were managing their own separation, the subjective workload ratings for the controllers were reported as high. That increase in workload appeared to be directly related to the increase in communication requirements necessary to accomplish the controllers' management of their airspace. Thus, the investigation emphasized the importance of providing tactical intent information to the controllers in free flight operations

There have also been some studies exploring the effects of free flight operations on flight crews. Collaborative studies conducted by NASA and the Netherlands National Aerospace Laboratory (Mackintosh et al., 1998) examined flight crew procedures in free flight operations. Each of the investigations examined the effects of traffic density on the flight crew participants. Crews were provided with prototypic airborne alerting logic and CDTI display tools to help enable the flight crew separation tasks. Both studies found longer conflict detection times in high density compared to low-density traffic scenarios. The NASA investigation also included controllers as participants. In that investigation, both flight crews and controllers appeared to have some performance differences based on the different geometry of the conflict angles.

Some flight deck research has also begun to explore the usefulness of free flight tools. Johnson, Battiste, and Bochow (1999) have provided some guidelines related to CDTI features that might be required in a free flight operational environment. Their research suggests the importance of color coding and 3-D flight plans for alerting and situation awareness. These features and their display characteristics may be critical to the successful implementation of shared-separation.

Another aspect of free flight that has been discussed in the literature is the characteristics of the airborne alerting logic. As a tool, this logic will assist the flight crews in detecting and resolving conflicts in a shared-separation environment. Cashion and Lozito (2000) examined the impact of different levels of intent in the airborne alerting logic on flight crews. They found that crews 
prefer longer-term intent [i.e., aircraft intent that includes horizontal and vertical navigation components of the Flight Management System (FMS)]. However, the flight crews expressed concern about display clutter when portraying more intent data on the CDTI.

Smith, Billings, McCoy, and Orasanu (1999) reviewed other free flight issues. Their findings suggested there may be advantages to allowing pilots to have additional tools available such as enhanced weather displays, conflict alert probes, and others. The research suggested that the decision-making process becomes increasingly complex as communications increase and more decision makers are placed into the loop. Controllers may become less efficient and less able to retain awareness of traffic situations if their management role is changed to the position of a monitor of a highly complex automated system. The research also showed that a key to improving operations may involve cooperative flight planning and the sharing of information concerning routine bottlenecks or constraints.

In summary, previous research showed the need to develop both tools such as CDTI and ground conflict probes and procedures to deal with issues unique to free flight. The research suggested that roles and responsibilities of both the pilots and controllers need to be clearly defined. The following is a list of free flight issues identified from those studies that may require further investigation:

- Impact on controller and pilot workload and situation awareness.

- Impact on communications due to exchange of information and inquiry.

- Ability of controllers to make timely interventions to resolve conflicts.

- Need for pilot intent information.

- Need for additional automation to assist with the management of information.

- Assessment and development of procedures. Development of decision support tools and displays.

- Impact of aircraft mix on operations.

- Integrated evaluation of the above.

This study was designed to address and investigate aspects of these issues (with the exception of the last one).

\section{METHOD}

Typically, fast-time simulation, modeling, paper studies, part-task and lower fidelity real-time human-in-the-loop simulation studies provide preliminary assessments of advanced concepts such as shared-separation. The literature review indicated that a number of such studies were done. However, the literature identified a scarcity of data from an air-ground integrated perspective. Although shared-separation concepts are not matured, the researchers felt that conducting a high fidelity simulation would identify a direction for further research and examine early feasibility and benefits from an integrated perspective. Therefore, researchers chose a high fidelity infrastructure. Additionally, by conducting simulation in high fidelity laboratories, the researchers attempted to eliminate the effect of nuisance variables. 


\subsection{Participants}

Participants included air traffic controllers and line pilots. Participants were organized in groups consisting of four controllers (a radar [R-side] controller and a radar associate [D-side] controller team per sector) and two line pilots (a flight crew).

\subsubsection{Controllers}

Three groups of Certified Professional Controllers (CPCs) and one group of Operations Supervisors (OSs) ${ }^{1}$ from Memphis ARTCC (ZME) participated in the simulation as air traffic controllers. Each group consisted of two, 2-member teams and participated for 3 days during the 4 weeks of simulation. Each sector was staffed with an R-side controller and a D-side controller. All CPCs and supervisors were qualified to control traffic in the sector and position they were assigned to operate. Their sector and position assignment did not change throughout the simulation. Table 1 summarizes the participant demographic information.

Table 1. Summary of Background Form Responses

\begin{tabular}{|l|c|c|c|c|}
\hline \multirow{2}{*}{ Characteristics } & \multicolumn{2}{|c|}{$\begin{array}{c}\text { Certified Professional } \\
\text { Controllers }(\mathbf{n = 1 2 )}\end{array}$} & \multicolumn{2}{c|}{$\begin{array}{c}\text { Operations Supervisors } \\
(\mathrm{n}=4)\end{array}$} \\
\cline { 2 - 5 } & $\mathbf{M}$ & $\mathbf{S D}$ & $\mathbf{M}$ & \multicolumn{1}{c|}{$\boldsymbol{S D}$} \\
\hline Age & 37.9 years & 3.2 years & 43.5 years & 4.2 years \\
\hline $\begin{array}{l}\text { Total experience as } \\
\text { Developmental-CPC / CPC }\end{array}$ & 14.9 years & 3.7 years & 19.6 years & 4.5 years \\
\hline $\begin{array}{l}\text { Experience as an FAA } \\
\text { Developmental-CPC / CPC }\end{array}$ & 14.1 years & 3.1 years & 17.8 years & 4.1 years \\
\hline Experience as a CPC & 11.9 years & 3.4 years & 15.9 years & 5.1 years \\
\hline Years at ZME & 12.6 years & 3.1 years & 15.5 years & 1.9 years \\
\hline Years of URET usage & 1.4 years & 0.1 years & 3.0 years & 0 years \\
\hline URET usage at ZME sector 21 & $92.5 \%$ & $9.9 \%$ & $100 \%$ & $0 \%$ \\
\hline URET usage at ZME sector 44 & $93.3 \%$ & $12.1 \%$ & $100 \%$ & $0 \%$ \\
\hline Overall URET usage & $86.7 \%$ & $12.6 \%$ & N/A & N/A \\
\hline
\end{tabular}

'Unfortunately CPCs were not available from the field for the last week of the study, therefore OSs acted as participants for one week. Data from the OSs were not included in data analyses except for background statistics and comments from their forms and debriefing sessions. 


\subsubsection{Pilots}

Three flight crews, consisting of both captains and first officers from a major United States airline served as participants. All pilots were either current on the Boeing 747-400 or retired for not more than 6 months. Pilots flying the Boeing 747-400 typically fly oceanic routes; therefore, all participants in this study were oceanic line pilots to avoid training concerns. The pilots flew in their normal crew position. The captains had a mean total flight time of 18,000 hours, and the first officers had a mean total flight time of 16,930 hours.

\subsection{Simulation Experiment Team}

The simulation experiment team consisted of two test directors (one at the William J. Hughes Technical Center [WJHTC] and one at NASA ARC), human factors researchers, subject matter experts, statisticians, laboratory personnel, and audio/video personnel from the FAA, NASA, and VNTSC.

The test directors were responsible for the overall management of the simulation and directed simulation-related activities of all members of the simulation experiment team.

Human factors researchers, subject matter experts, statisticians, and trained simulation pilots staffed the experiment team positions. Experiment team members also administered forms and conducted participant briefings and debriefings. Laboratory personnel operated, monitored, and maintained the laboratory systems used in the simulation. All team members were available in the test areas to support the test directors.

\subsubsection{Expert Observers}

Two expert observers (EOs) participated as part of the simulation experiment team during each of the 4 study weeks. One EO observed Sector 21 and the other observed Sector 44 . EOs were subject matter experts in the field of ATC.

\subsubsection{Ghost Sector Controller}

Two members of the simulation experiment team staffed the "ghost sector" controller position for all adjacent, non-simulated sectors. The ghost controllers accepted and made hand-offs and performed air $\leftrightarrow$ ground and ground $\leftrightarrow$ ground (land line) communications as required. These individuals were trained on the necessary equipment, airspace, and sector operating procedures.

\subsubsection{Automatic Datalink Operator}

One member of the simulation experiment team staffed the automatic datalink operator (ADO) position during specific runs as appropriate. Two individuals were trained for the ADO position and alternated throughout the study. The ADO had prior knowledge of all scripted altitude and course changes and monitored the air $\leftrightarrow$ air and test director frequencies for any unexpected/ unknown altitude or course changes. The ADO, situated at a separate Display System Replacement (DSR) console, updated the Host computer with the required changes. This ensured that the Host, CDTI-AL, and URET remained current and consistent with flight plan updates. 


\subsubsection{WJHTC Simulation Pilots and Laboratory Coordinators}

Ten trained WJHTC simulation pilots and two laboratory coordinators from the simulation experiment team supported the two-sector operation. Simulation pilots emulated pilot communications and actions. They initiated scripted air $\leftrightarrow$ air and air $\rightarrow$ ground communications and responded to ATC instructions. The simulation pilots also entered data into the desktop simulators as required by the scripts and in response to controller-issued instructions (e.g., turn right heading 120, climb to and maintain Flight Level (FL) 270, etc). Eighty percent of the simulation pilots were licensed or retired pilots.

\subsubsection{Intruder Simulation Pilot}

One trained simulation pilot at NASA ARC staffed the intruder aircraft simulator that was scripted to be involved in planned conflicts ${ }^{2}$. The intruder simulation pilot was trained on the use of CDTI-AL and the right-of-way rules. This individual also had access to communications with both the controllers and flight crew participants.

\subsection{Facilities and Equipment Overview}

The simulation test bed integrated facilities and equipment from both the WJHTC and NASA ARC. WJHTC facilities included: Integration and Interoperability Facility $\left(I^{2} F\right)$, Target Generation Facility (TGF), and the Pseudo Aircraft Systems (PAS) laboratory. The I F equipment configuration included: Host processor, DSR consoles, URET, voice communication system, audio and video recording system, and workload assessment keypads (WAKs). The NASA ARC facility used was the Crew-Vehicle Systems Research Facility (CVSRF). The CVSRF laboratory and equipment configuration included: NASA ARC Boeing 747-400 flight simulator (NASA ARC simulator), alerting logic, flight crew displays and tools, PAS laboratory, intruder aircraft simulator, voice communication system, and audio and video recording system.

The WJHTC and NASA ARC laboratories were linked across the country via a high-speed circuit (fractional $\mathrm{Tl}$ line) that digitally transmitted data and voice. Figure 1 depicts the integration of the facilities.

\footnotetext{
${ }^{2}$ A planned conflict was defined as two aircraft on flight paths that would collide if there was no corrective action taken.
} 


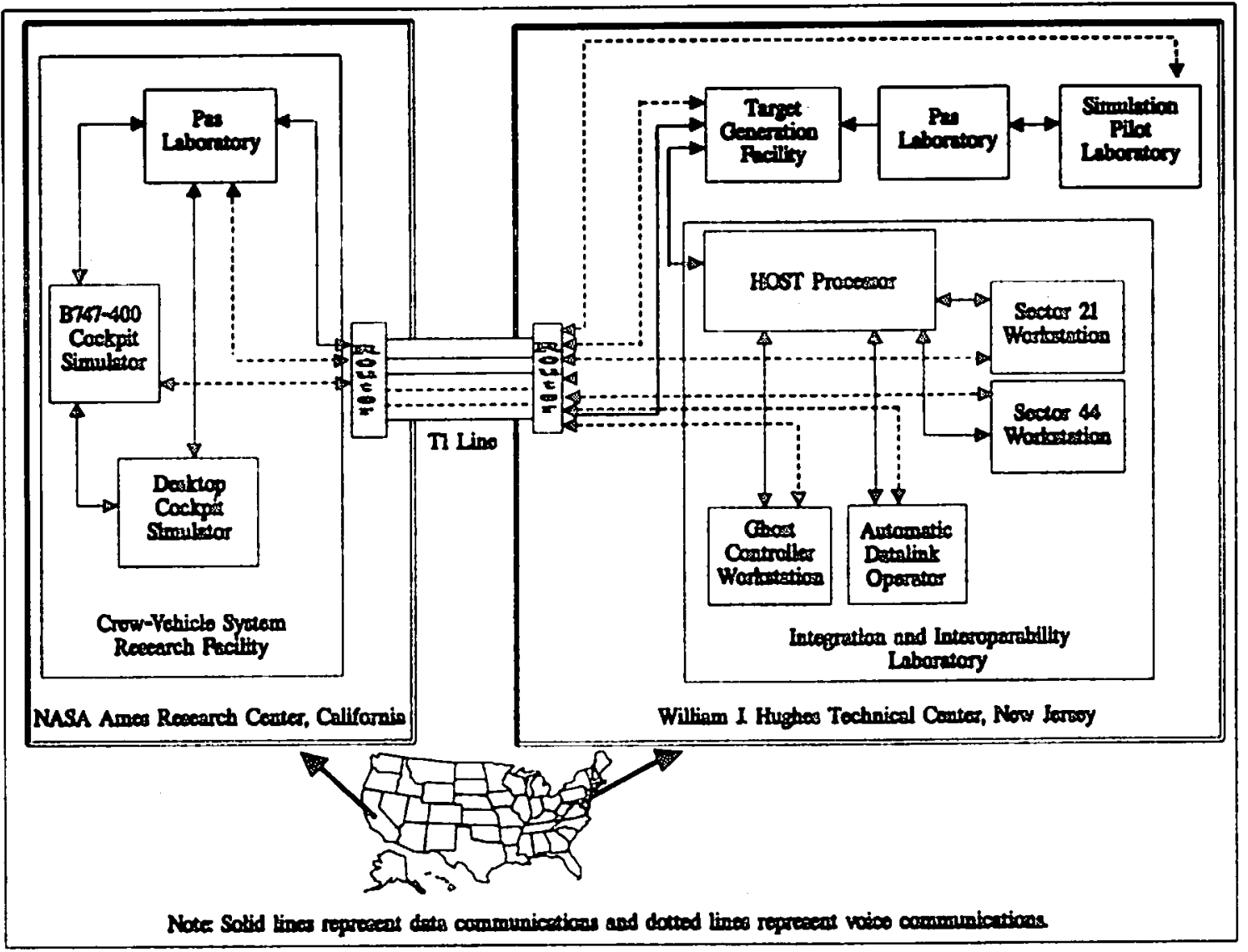

Figure 1. FAA and NASA facilities used in AGIE.

\subsubsection{WJHTC Facilities and Equipment}

\subsubsection{Integration and Interoperability Facility}

The $I^{2} F$ is devoted to exploring the issues associated with modernizing the NAS infrastructure. The $I^{2} F$ provides a realistic DSR en route environment for research. It is open to modifications of both hardware and software to facilitate the conduct of engineering evaluations. The $I^{2} \mathrm{~F}$ is designed for prototype experimentation, system-level integration, proof of concept evaluations (i.e. shared-separation operations), and interoperability verification and evaluations. The $I^{2} F$ sector configuration for this experiment is depicted in Figure 2. 


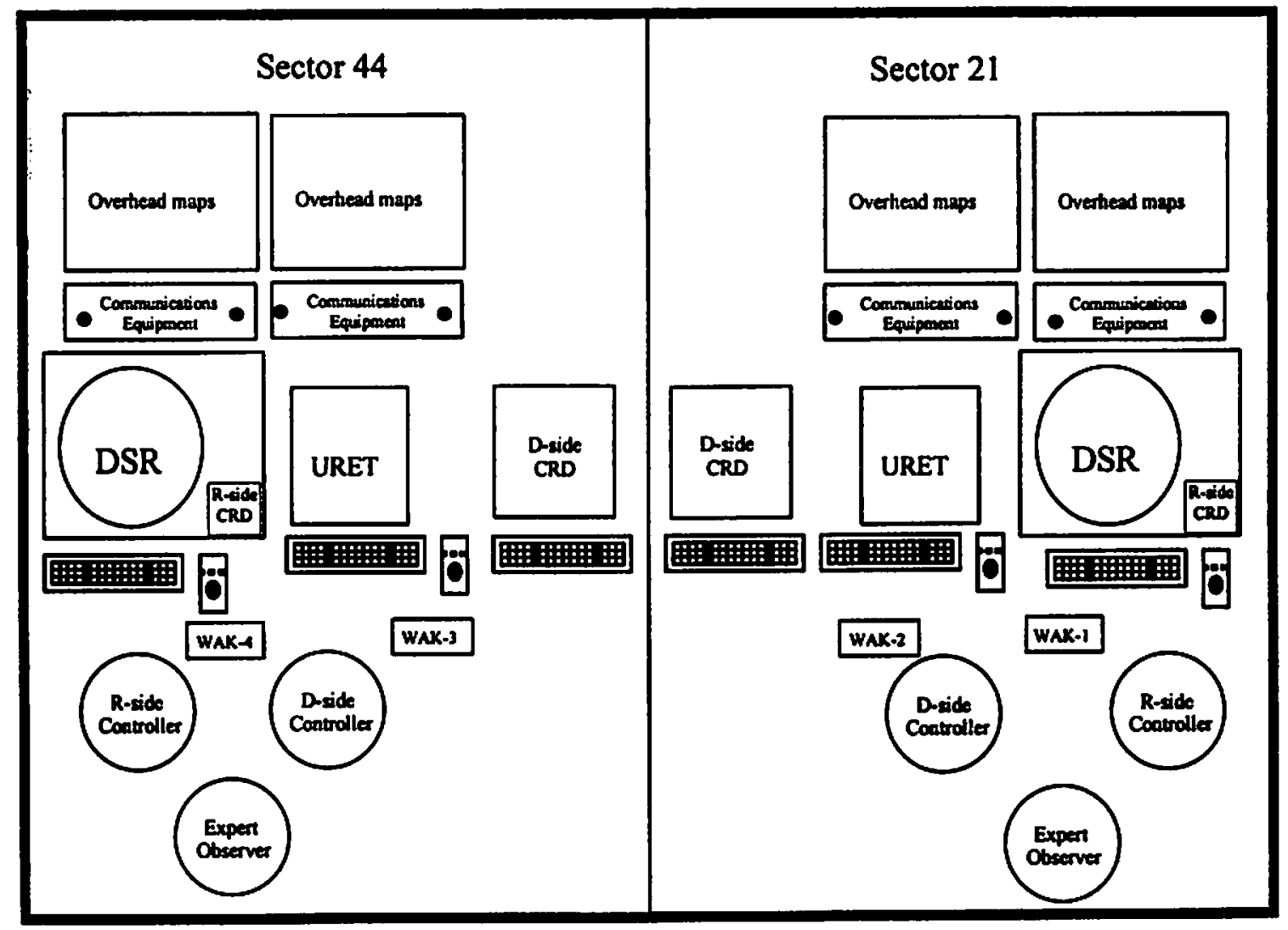

Figure 2. $\mathrm{I}^{2} \mathrm{~F}$ controller workstation and sector layout.

- Display System Replacement

DSR consoles replaced existing en route display systems with new hardware and software at the ARTCC. DSR provided Common Console Workstations on high-resolution 20 inch by 20 inch color display screens. DSR utilizes Reduced Instruction Set Computer processing technology and serves as a platform for future ATC system upgrades. The DSR version used in this experiment was BABO3.

- User Request Evaluation Tool

URET was developed to assist the ARTCC controller in predicting and evaluating potential conflicts between aircraft. URET is currently installed as a prototype system and fielded for daily use at the Memphis and Indianapolis ARTCCs. The system functionality consists of trajectory modeling, conformance monitoring and reconformance, current plan and trial plan processing, automated problem detection, interfaces with the Host and external data sources, and computer-human interface.

URET provides the controller five levels of automated problem detection alerts with a "lookahead" time of approximately 20 minutes. The following alerts are presented to the controller in both tabular and graphic form: 
- RED Alert - The alert given if aircraft are predicted to pass within the standard separation limits of five nm horizontally and $1000 / 2000 \mathrm{ft}$ vertically (as appropriate ${ }^{3}$ )

- Muted RED Alert - The alert given if the predicted separation between two aircraft is less than the standard separation limits, and the separation loss is predicted to occur on a portion of the route where an altitude transition is planned, but not yet cleared.

- YELLOW Alert - The alert given if aircraft are predicted to pass between 5 to 10 miles horizontally and within 1000/2000 ft vertically (as appropriate).

- Muted YELLOW Alert - The alert given if the predicated separation between two aircraft is between 5 to 10 miles horizontal, less than $1000 / 2000 \mathrm{ft}$ vertical, and the separation loss is predicted to occur on a portion of the route where an altitude transition is planned, but not yet cleared.

- BLUE Alert - The alert given if aircraft are predicted to enter Special Use Airspace (SUA) based on their current trajectory.

The URET version used for this study was D32. This version provided a two-way Host interface allowing controllers to amend flight plans directly through URET. To mimic operations in the field, paper flight strips were not provided to participants. (URET information was used in lieu of the flight strips.)

- Voice Communication Systems

The $I^{2} \mathrm{~F}$ and $\mathrm{CVSRF}$ integrated their laboratory voice communication and recording systems to emulate the operational ATC and flight deck systems in use today.

Voice communications between the facilities were transmitted over a leased digital circuit (fractional T1). This circuit carried voice information in Internet Protocol (IP) packets. One Cisco 3640 Voice Over IP router was located at each end of the T1 circuit and provided an interface between the individual voice communications systems and the $\mathrm{T} 1$.

The system provided six separate voice frequency channels for the simulation. These included an air $\leftrightarrow$ air channel, air $\leftrightarrow$ ground channels for each of the two sectors simulated and the ghost sector, a land line channel for all sectors, and an additional channel for communications between test directors at NASA and WJHTC.

Pilots were able to transmit on the air $\leftrightarrow$ air and air $\leftrightarrow$ ground frequencies. Pilots could neither monitor nor transmit on the land line channels. Controllers were able to transmit on the land line and air $\leftrightarrow$ ground frequencies. Controllers were able to monitor but not transmit on the air $\leftrightarrow$ air channel. The controllers and pilots used their headphones to access all frequencies; no loud speaker or other equipment was provided. Controllers and pilots were able to simultaneously monitor both the air $\leftrightarrow$ air channel and their specific air $\leftrightarrow$ ground channels.

\footnotetext{
${ }^{3}$ Vertical separation requirements for aircraft flying below flight level (FL) 290 is $1000 \mathrm{ft}$. Vertical separation requirements for aircraft nying above FL290 is $2000 \mathrm{ft}$.
} 
- ATC Audio and Video Recording System

A mobile recording system was used to record the audio and video data during each simulation run. ${ }^{4}$ Four black and white, low-light micro cameras recorded two views of each sector. One camera was focused on a general sector overview, and the second camera was focused on the URET display for each sector. All videos were recorded in the Super VHS format on tapes stamped with National Television System Committee linear time code for synchronous playback purposes.

Ambient communications were recorded from wireless microphones worn by each controller. Land line, air $\leftrightarrow$ ground, and air $\leftrightarrow$ air voice communications were separately recorded. All audio and video signals were mixed using a Tascam M2516 audio mixing board and recorded on the hi-fi audio channels of the videotapes.

- Workload Assessment Keypad

A WAK was provided to each controller position. Using the WAK, instantaneous controller workload ratings on a 1-to- 5 scale $(1=$ very low, $3=$ moderate, and $5=$ very high $)$ were collected at 5-minute intervals. Four WAK units were connected to one laptop. This laptop hosted the WAK software and recorded the data entered. The software emitted a low level beep every five-minutes on all four WAKs simultaneously. At the same time, the keys were illuminated for a maximum of 20 seconds. If a participant did not enter a workload rating in 20 seconds, the WAK automatically recorded an entry of 99 to indicate missing data. Figure 3 depicts the WAK.

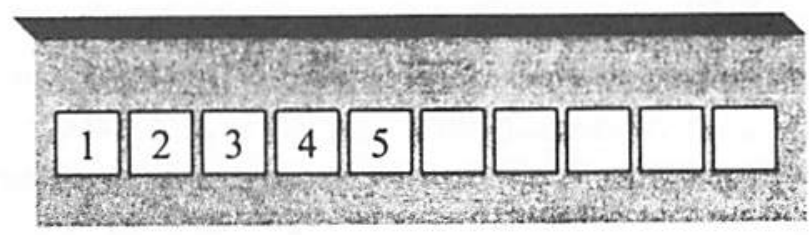

Figure 3. Workload assessment keypad.

\subsubsection{Target Generation Facility}

The TGF generated high fidelity digital radar messages for targets in the simulated airspace environment. The messages were adapted to mimic actual NAS characteristics by including the radar and environmental characteristics of the ZME. Simulated primary and beacon radar data were generated for each target and processed by the multiple radar processing function of the

\footnotetext{
${ }^{4}$ Video and audio recordings were exclusively used to backup information obtained by other means. They also provided a mechanism to explore issues that may have been unclear in the objective and subjective data obtained during this simulation. The information contained on these tapes was not be used for any other purpose. All tapes, so obtained, are held by ACT- 540 and were made available only to the members of the experiment team and to personnel designated by NATCA. All tapes were destroyed following publication of the final report.
} 
NAS in a manner similar to normal radar data. Flight data blocks contained the flight identification, beacon code, and altitude. Target positions were automatically updated at the same rate that is experienced in the ARTCC. To simulate actual aircraft operations, the radar targets maneuvered based on route segments from a flight plan and by the actions of the simulation pilots and participant pilots.

\subsubsection{Pseudo Aircraft Systems Laboratory}

PAS is a computerized flight dynamics and piloting system designed to provide a high fidelity, multi-aircraft, and real-time simulation environment to support ATC research. PAS is comprised of three major software components that run on a network of computer workstations: the Simulation Manager, the PAS Pilot Manager, and the Pilot Station Laboratory. Combined, the components simulated the following functions: aircraft performance characteristics and flight dynamics, flight plans, aircraft state information, and display and control capabilities for the simulated aircraft on a set of workstations. Both NASA and the WJHTC used PAS laboratories with the same version of PAS software (version pas_4.3.2) in their laboratories. The WJHTC PAS laboratory had twelve pilot workstations configured for AGIE. Ten workstations were for WJHTC simulation pilots and two were for laboratory coordinator positions.

\subsubsection{NASA Crew-Vehicle Systems Research Facility and ARC Simulator}

The CVSRF is a unique national research facility dedicated to studies of aviation human factors and airspace operations and their impact upon aviation safety. An integral component of the CVSRF is the NASA ARC simulator.

The NASA ARC simulator was built by CAE Electronics and certified to the FAA Level D certification requirements (Sullivan \& Soukup, 1996). The Boeing $747-400$ has an advanced level of automation available to the pilots. The visual system uses photo texturing and offers superior scene quality, depicting out the window scenes in night, day, dusk, or dawn conditions. In addition, the simulator has an advanced digital control loading and a six degree-of-freedom motion system. Features added to the simulator to support this research included new display components (CDTI) and the input control devices for the display (see Figure 4). Data collection is available for user interaction with all subsystems, including the autopilot system and communication devices. 


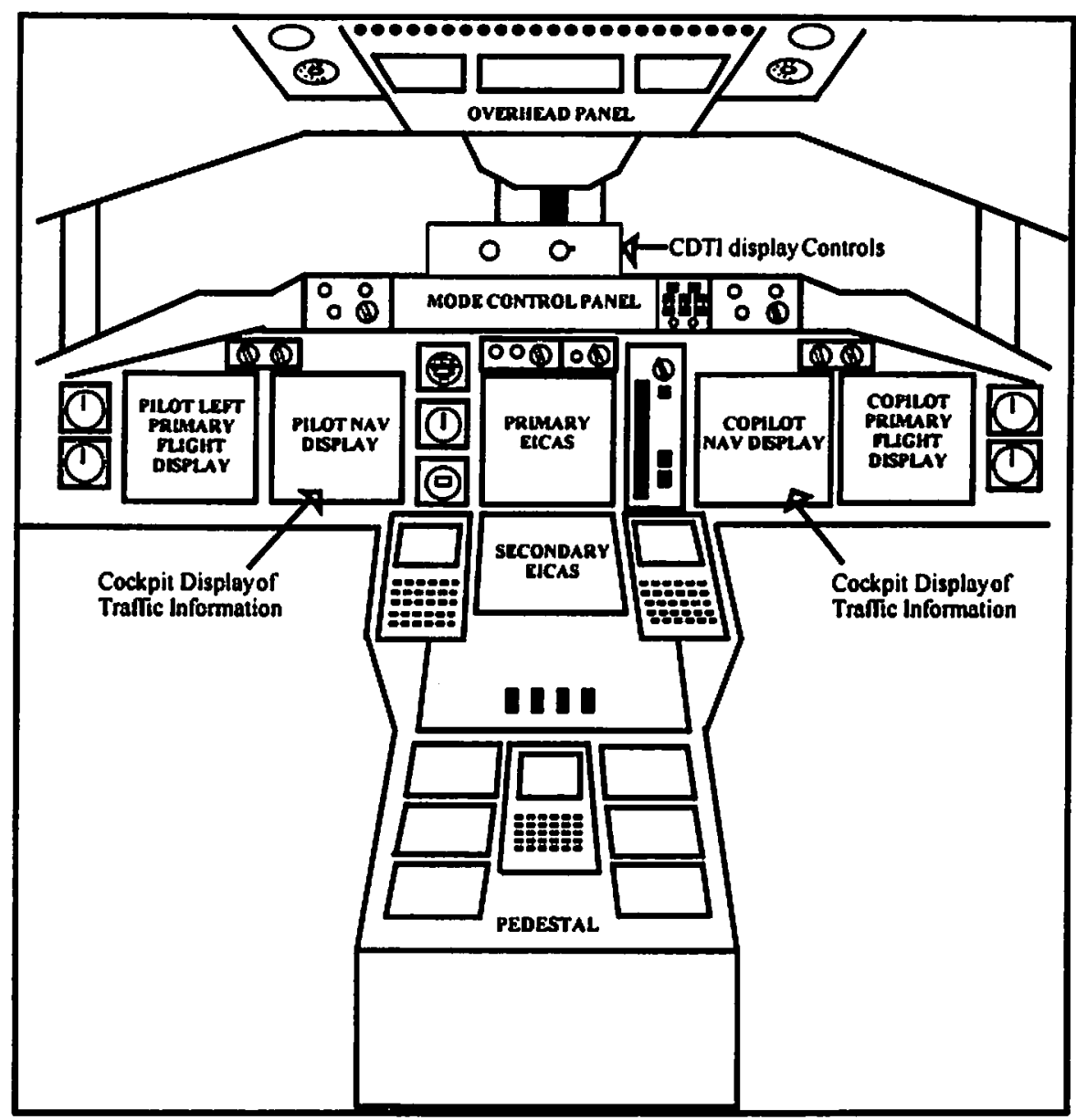

Figure 4. NASA ARC simulator flight deck layout.

\subsubsection{Alerting Logic}

This study included a prototype airborne alerting logic designed to aid in shared-separation operations (Yang \& Kuchar, 1997). This alerting logic overlaid the NASA ARC simulator's Traffic Alert and Collision (TCAS) logic. TCAS involves immediate tactical conflict avoidance whereas the new airborne alerting logic was designed to help flight crews manage the more strategic shared-separation responsibilities. The goal was to create a seamless relationship between the airborne alerting logic and TCAS (see Figure 5). 


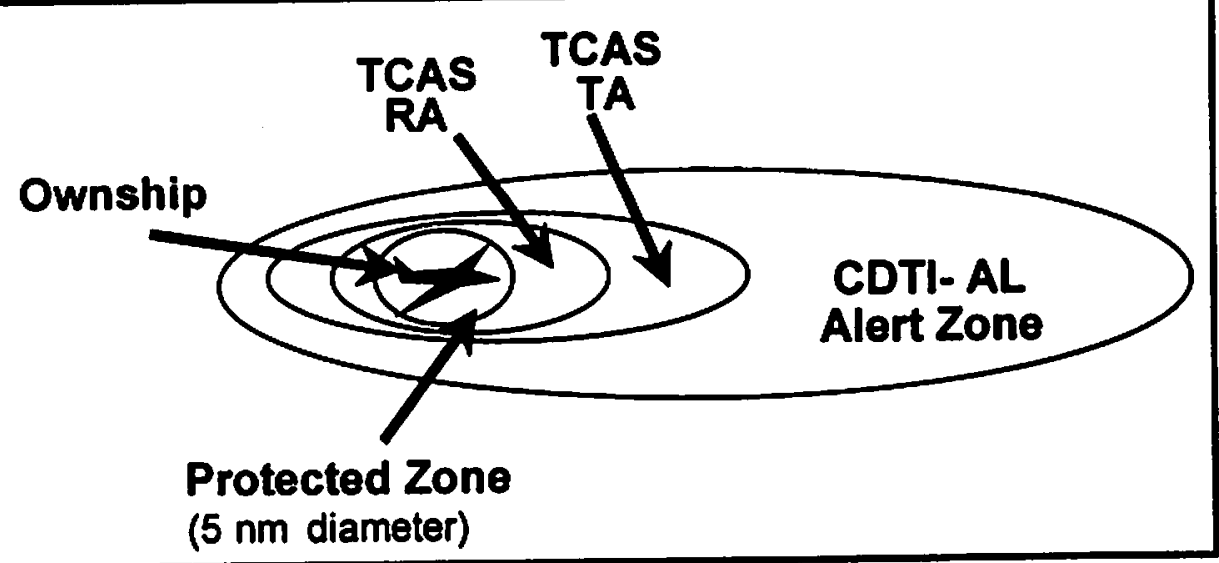

Figure 5. Relationship of new airborne alerting logic to TCAS logic.

Therefore, TCAS was left intact with the exception that the first two threat levels of display symbology (unfilled diamond and filled diamond) were replaced with the experimental display symbology. The yellow circle for a Traffic Advisory (TA) and a red square for a Resolution Advisory (RA) were still available. Currently, the TCAS display depicts surrounding traffic up to $40 \mathrm{~nm}$ from the NASA ARC simulator on the navigation display. In contrast, the alerting logic in this study extended traffic depiction out to $120 \mathrm{~nm}$ in front of and to each side of the NASA ARC simulator and $30 \mathrm{~nm}$ behind the NASA ARC simulator based on the expected ADS-B surveillance capabilities (RTCA, 1992). Additionally, based on expected ADS-B capabilities, the update rate for the navigation display was once per second. To reduce clutter, an altitude filter limited the vertical range of viewable traffic to $4100 \mathrm{ft}$ above and below the NASA ARC simulator.

The airborne alerting logic provided an additional alerting zone beyond that of TCAS. The alert was provided to the flight crews. A CDTI-AL alert was triggered for the flight crews when the alerting logic predicted a pending violation of the protected zones (or minimum separation requirement) of the aircraft (Yang \& Kuchar, 1997). Operationally, the CDTI-AL alert is the point at which intervention may be required (RTCA, 1995).

\subsubsection{Flight Crew Displays and Tools}

Traffic was represented on the flight deck navigation display by the symbol " $V$ " with the apex indicating the aircraft direction. Altitude was pilot selectable as altitude relative to the NASA ARC simulator or absolute altitude. All traffic was initialized as non-threat aircraft. In addition, all new display features for non-conflicting aircraft were in white. Figure 6 depicts all aircraft in a non-threat status. 


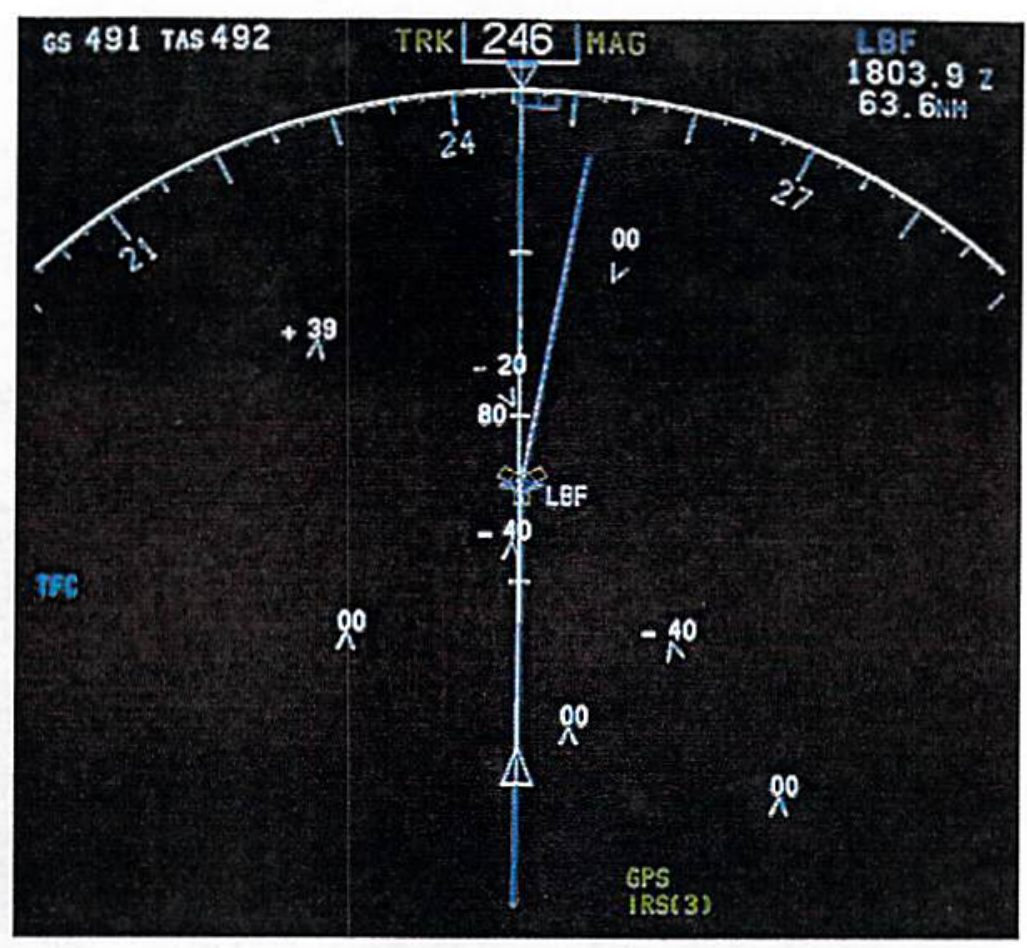

Figure 6. CDTI-AL depicting non-threat aircraft.

When the probability of a violation of the protected zone increased, a CDTI-AL alert was indicated to the flight crew by

- a blue line extending from both the NASA ARC simulator and the intruder aircraft symbols. At the end of each line was a blue circle that represented the current separation standard of 5 $\mathrm{nm}$ in diameter. Any overlap of the circles indicated impending loss of horizontal separation.

- an aural warning "alert" sounded twice;

- the word "ALERT" appeared in blue on the lower right hand corner of the display along with the intruder aircraft call sign and the time to minimum separation distance (MSD).

The time to MSD was the time remaining before aircraft were projected to pass in closest proximity to each other on current flight paths. All display features associated with the aircraft involved in a CDTI-AL alert (aircraft symbol, altitude, ground speed, and callsigns) as well as the display changes related to an alert appeared in blue to help identify which aircraft were predicted to conflict. Figure 7 illustrates the display changes associated with a CDTI-AL alert. As flight crews solved a conflict, the alert level degraded to a non-threat status as the threat probability was reduced.

Flight crews also could select certain display features designed to aid them in shared-separation responsibilities. Selectable display features could be manipulated by a small box mounted above the Mode Control Panel (see Figure 8). Flight crews could reduce clutter by toggling a button to de-select the traffic callsigns. Another selectable feature was the temporal predictor. The 


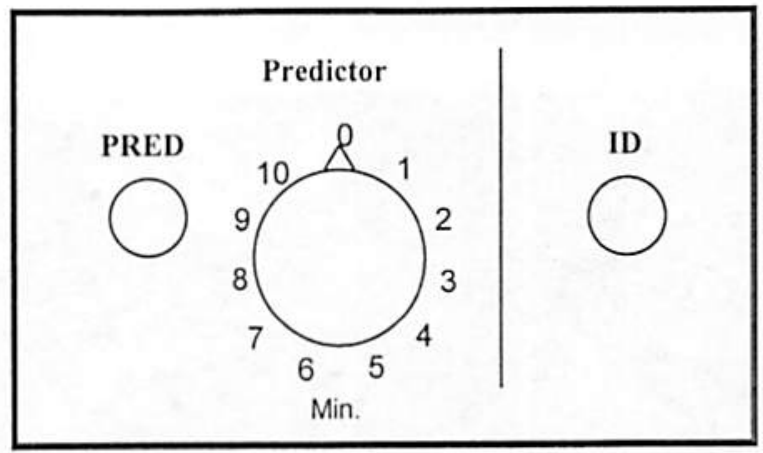

Figure 7. CDTI-AL depicting an alert.

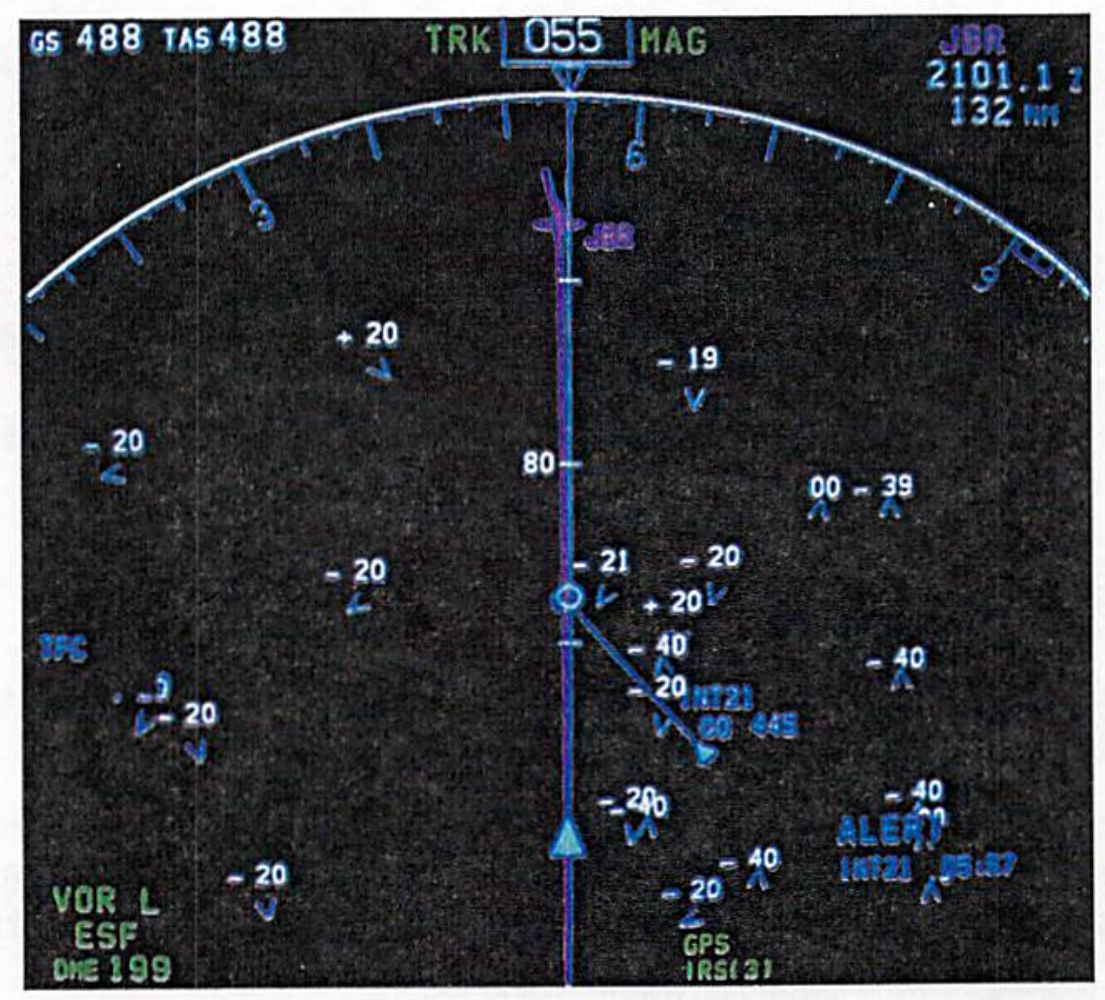

Figure 8. Control box for pilot selectable features.

predictor provided crews with an estimation, based on current aircraft state information, of where other aircraft would be relative to the NASA ARC simulator up to 10 minutes into the future. The selection knob for the temporal predictors allowed crews continuous control of the predictor length from 0 to 10 minutes at 1 second intervals. Although predictor manipulation does not invoke the alerting logic, crews could visually determine which aircraft might create a potential conflict prior to an alert level indication. When predictors were selected, they were displayed for all aircraft (see Figure 9). The predictor symbol was identical to the shape of the CDTI-AL alert symbology with a line and a circle that represented $5 \mathrm{~nm}$ in diameter, except that the predictor symbology was white, and the alert symbology was blue. Selected predictor time was displayed at the lower right hand corner of the navigation display. In addition, to reduce clutter, predictors and callsigns of the non-conflicting traffic were automatically cleared from the display at the onset of a CDTI-AL alert but could be reselected at any time. 


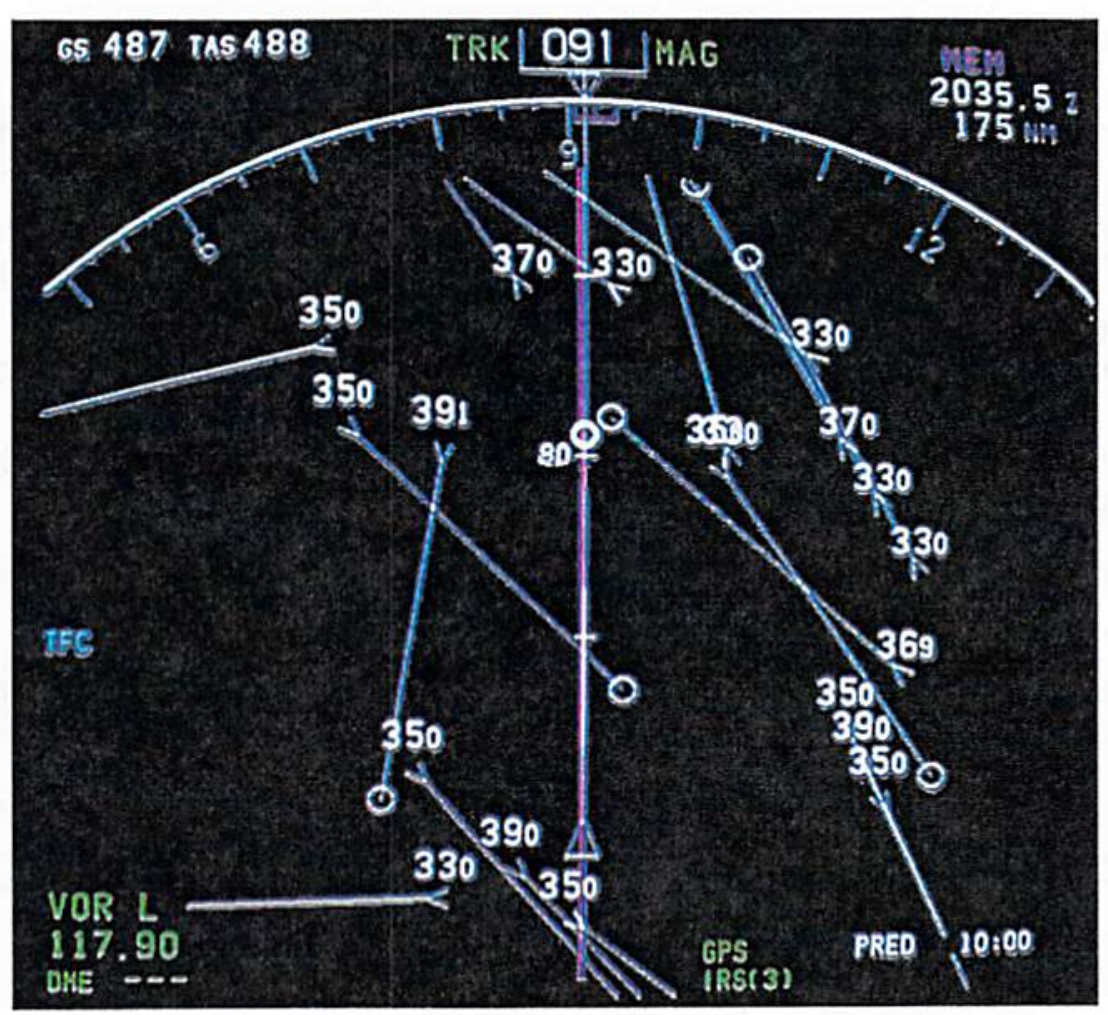

Figure 9. CDTI-AL depicting predictors selected.

Finally, pilot participants could also de-clutter the navigation display by changing the horizontal map range. Ranges available were similar to those available on the navigation display on most Boeing 747-400 aircraft $(10,20,40,80,160,320$, and $640 \mathrm{~nm})$.

\subsubsection{PAS Laboratory and Intruder Aircraft Simulator}

The PAS laboratory at NASA ARC was the same system employed by the WJHTC. NASA $\mathrm{ARC}$ had one workstation configured as the intruder aircraft simulator. The intruder aircraft supplied the simulation pilot with the same display of traffic and airborne alerting logic as the pilot participants. The workstation also supplied the ZME display of traffic.

\subsubsection{NASA Audio and Video Recording}

There were three cameras within the NASA ARC simulator that provided views of the flight deck and the CDTI-AL ${ }^{5}$. In addition, the microphones within the simulator allowed for recording of all air $\leftrightarrow$ air and air $\leftrightarrow$ ground communications, along with communications within the cockpit.

\footnotetext{
${ }^{5}$ Video and audio recordings were used to provide a mechanism to explore issues that may have been unclear in the objective and subjective data obtained during this simulation. The information contained on these tapes was not used for any other purpose. All tapes, so obtained, are held by NASA ARC and were made available only to the members of the experiment team.
} 


\subsection{Airspace}

Two ZME sectors, sectors 44 and 21, were emulated in the experiment. All adjacent surrounding sectors were combined on a single position and collectively referred to as sector 78 (ghost sector). Sector 78 was staffed by an experiment team member for realism. Figure 10 depicts the two sectors selected for the study and all adjacent sectors. ZME airspace was chosen because it is currently one of the locations where DSR and URET have been operationally fielded. The sectors were selected based on recommendations from ZME personnel for the following reasons:

- Both sectors 21 and 44 are high altitude sectors that contain moderate to high traffic flows producing moderate to high workload.

- The sectors are considered to be of moderate to high complexity (subjectively described by ZME).

- The sectors are adjoining, therefore presenting the opportunity to observe inter-sector coordination.

- The sectors are from different areas of specialization. This eased the impact on field staffing while they participated in this research endeavor.

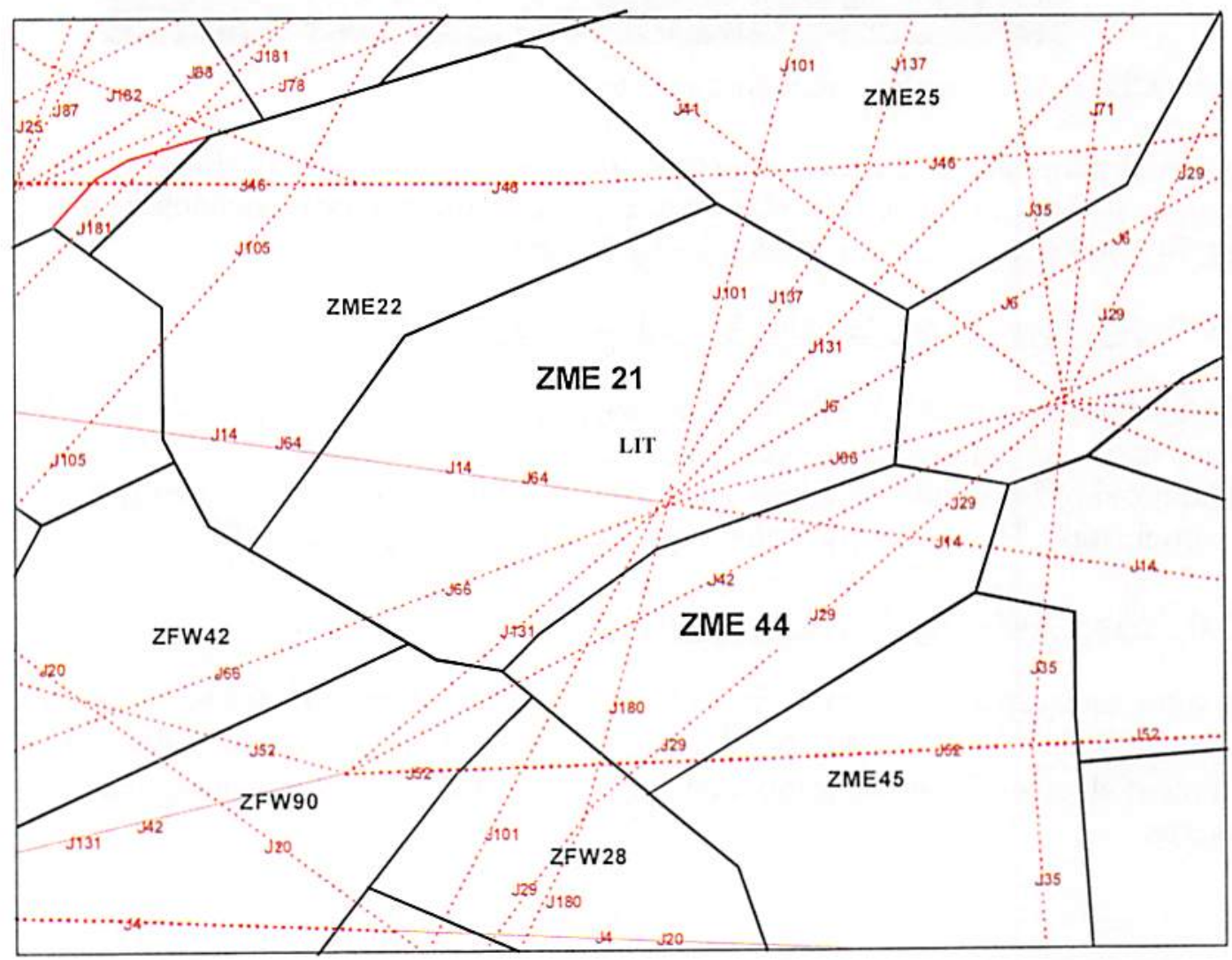

Figure 10. ZME Sectors 21 and 44. 


\subsubsection{Sector 21 (Conway High)}

Sector 21 (Conway High) is a ZME high altitude sector encompassing the airspace between FL 240 and FL310. The focal point for the route segments within the sector is the Little Rock (LIT) Very High Frequency Omni Directional Radio Range (VOR) Tactical Air Navigational Aid (VORTAC). This is the only navigational aid in the sector and approximately 11 jet route segments converge over it. The sector's location and roughly rectangular shape is such that it is bounded on the southwest by Fort Worth Center, on the northwest by the Razorback High sector, on the northeast by Blytheville High sector, on the east by the Memphis High sector, and on the south by the Pine Bluff High sector. The sector is approximately $190 \mathrm{~nm}$ from northeast to southwest and $80 \mathrm{~nm}$ from north to south. In the simulation, the sector was emulated as described, except that the airspace was expanded to include FL240 and all altitudes above.

\subsubsection{Sector 44 (Pine Bluff High)}

Sector 44 (Pine Bluff High) is a ZME high altitude sector encompassing the airspace of FL240 and above. The sector is one of seven high/ultra high sectors within the ARTCC. The sector's location and rectangular shape starts southwest of LIT VORTAC and proceeds northeast for 135 miles, turns south-southwest for 50 miles, turns southwest for 100 miles then intercepts the Dallas/Fort Worth ARTCC boundary. From that point, the sector proceeds northwest for 50 miles to the point of its beginning. In the simulation, the sector was emulated as described.

\subsection{Experimental Conditions}

The simulation experiment consisted a four conditions defined by various levels of controller and flight crew shared-separation responsibilities. The conditions were Current Operations (CO), CO with CDTI-AL (CO:CDTI), Shared-Separation Level 1 (SS:L1), and Shared-Separation Level 2 (SS:L2). Each condition used a different set of procedures that reflected their changing roles and responsibilities. Current standard separation rules of $5 \mathrm{~nm}$ horizontal or $1000 / 2000 \mathrm{ft}$ vertical as appropriate were observed for all conditions. A within-subjects design was utilized where all flight crew and controller participants were exposed to each condition.

- $\mathrm{CO}$

This condition represented current ATC environment. URET was operational. Standard air traffic procedures defined in the Controller Handbook 7110.65 (FAA, 2000), Federal Aviation Regulations (FAR) Part 91 (FAA, 1997), and the Aeronautical Information Manual were applied during this condition. The pilots in this condition did not have access to the CDTI.

\section{- CO:CDTI}

This condition emulated elements of the RTCA (1995) definition of the free flight environment. This condition simulated all equipment and procedures of $\mathrm{CO}$ with the following changes: 
- Pilots had access to a CDTI-AL.

- $\quad$ Pilots could query controllers (e.g., regarding potential conflicts or traffic) and make requests based on information from their CDTI-AL displays to maximize efficiency or for safety concerns.

- SS:Ll

This condition emulated elements of the RTCA (1995) definition of the free flight environment. This condition simulated all equipment and procedures of CO:CDTI with the following changes:

- All flight crews started SS:Ll responsible for their own separation (i.e., free flight).

- All initial flight plans and altitudes were considered as optimum for the current conditions.

- Flight crews were free to initiate any maneuver (i.e., change heading, altitude, speed, or any combination) provided they first inform ATC.

- Flight crews were able to communicate with other flight crews on the air $\leftrightarrow$ air frequency. Controllers could monitor the air $\leftrightarrow$ air frequency as desired, but it was not required.

- Flight crews were instructed to use specific right-of-way rules to resolve conflicting situations.

- Flight crews could cancel free flight ${ }^{6}$ of their own aircraft at any time.

- Controllers were instructed to resolve any disputes between pilots.

- Controllers were instructed to issue rraffic $_{\text {alerts }}{ }^{7}$ to the aircraft involved in a URET RED alert.

- Controllers were instructed to coordinate all traffic alerts on aircraft not under their control with the controlling sector. Controllers receiving a coordinated traffic alert were instructed to forward this to the subject aircraft unless that aircraft had already advised that a resolution was in progress.

- Controllers could wait to issue a traffic alert until the subject aircraft was under their control.

\footnotetext{
${ }^{6}$ For the procedures of this study, the cancellation of free flight was defined as the cancellation of shared-separation operations resulting in aircraft separation responsibility switching from pilots (air) back to controllers (ground).

'For the procedures of this study, traffic alert was defined as an advisory that an aircraft was involved in a URET RED alert.
} 
- Controllers could only cancel free flight (for one or a pair of aircraft) if they had queried, or had knowledge of the intentions of, at least one of the aircraft.

- Sector-wide cancellation of free flight was NOT allowed.

- To issue a control instruction to a flight crew, controllers were instructed to first cancel free flight for that aircraft.

- Flight crews whose free flight had been canceled remained under ATC control unless/until the controller resumed free flight.

- Only controllers could resume free flight.

- SS:L2

This condition emulated elements of the RTCA (1995) definition of the free flight environment. This condition simulated all equipment and procedures of SS:Ll except for the following changes:

- Flight crews were not required to inform the controller before initiating any maneuver.

- Controllers were not required to issue traffic alerts to aircraft, but could do so. (However, they were still required to coordinate all URET RED alerts on aircraft not under their control with the controlling sectors).

- Controllers were not required to update the Host for altitude or flight plan amendments in this condition (the ADO emulated automatic datalink updates to the Host).

- Controllers could not cancel free flight for any aircraft at any time.

Table 2 summarizes characteristics of the experimental conditions.

\subsubsection{Scenario Development}

Three base scenarios were developed from flight plans extracted from ZME System Analysis and Recording (SAR) tapes and accompanying Adaptation Control Environment System configuration tapes obtained from the field. ZME personnel assisted in the development and modification of the scenarios. 
Table 2. Experimental Condition Characteristic Summary

\begin{tabular}{|c|c|c|c|c|}
\hline Characteristics & $\mathrm{CO}$ & CO:CDTI & SS:L1 & SS:L2 \\
\hline $\begin{array}{l}\text { Separation standards of } 5 \mathrm{~nm} \text { horizontal or } \\
1000 / 2000 \mathrm{ft} \text { vertical }\end{array}$ & $\sqrt{ }$ & $\sqrt{ }$ & $\checkmark$ & $\sqrt{ }$ \\
\hline URET was available to controllers & $\sqrt{ }$ & $\sqrt{ }$ & $\sqrt{ }$ & $\sqrt{ }$ \\
\hline $\begin{array}{l}\text { Controllers coordinated URET red alerts with other } \\
\text { sectors }\end{array}$ & $\sqrt{ }$ & $\sqrt{ }$ & $\sqrt{ }$ & $\sqrt{ }$ \\
\hline Controllers had full separation responsibility & $\sqrt{ }$ & $\sqrt{ }$ & & \\
\hline $\begin{array}{l}\text { Pilots required to request clearance from controllers } \\
\text { prior to maneuvering }\end{array}$ & $\sqrt{ }$ & $\sqrt{ }$ & & \\
\hline CDTI-AL was available to pilots & & $\sqrt{ }$ & $\sqrt{ }$ & $\sqrt{ }$ \\
\hline $\begin{array}{l}\text { Pilots and controllers shared-separation } \\
\text { responsibility }\end{array}$ & & & $\bar{V}$ & $\overline{\sqrt{ }}$ \\
\hline Air $\leftrightarrow$ air frequency was available & & & $\sqrt{ }$ & $\sqrt{ }$ \\
\hline $\begin{array}{l}\text { Pilots used right-of-way rules while resolving } \\
\text { potential conflicts }\end{array}$ & & & $\sqrt{ }$ & $\checkmark$ \\
\hline Pilots could cancel free flight & & & $\sqrt{ }$ & $\sqrt{ }$ \\
\hline Controllers could cancel free flight & & & $\sqrt{ }$ & \\
\hline $\begin{array}{l}\text { Pilots could initiate any maneuver but were required } \\
\text { to first inform controllers prior to maneuvering }\end{array}$ & & & $\sqrt{ }$ & \\
\hline $\begin{array}{l}\text { Controllers were required to issue traffic alerts to } \\
\text { aircraft concerning URET red alerts }\end{array}$ & & & $\sqrt{ }$ & \\
\hline $\begin{array}{l}\text { Pilots did not have to inform controllers prior to } \\
\text { maneuvering }\end{array}$ & & & & $\sqrt{ }$ \\
\hline
\end{tabular}

The data allowed for the realistic representation of sector boundaries, jet routes, and fixes for the simulated sectors. To suit simulation needs, the traffic scenarios were modified by altering some traffic flows, creating planned conflicts, and by increasing the number of aircraft to compensate for other complexity limitations. To control extraneous factors in the evaluation, the traffic scenarios did not include severe weather, and emergency or critical situations. In addition, there were very few ascending or descending aircraft to particularly avoid excessive URET-muted alerts that might detract from the evaluation. 
One of the base scenarios was used to create ATC training runs. The other two base scenarios were used to create data collection runs. These two base scenarios were slightly modified ${ }^{8}$ to create four unique traffic situations for the four conditions presented to the controllers and pilots. Specifically, CO and SS:L1 were created from the same base scenario, and CO:CDTI and SS:L2 were created from the same base scenario.

\subsubsection{Traffic Scenario Characteristics}

The experiment consisted of three training runs and four data collection runs that reflected varying levels of ATC and shared-separation operations. The scenario used to create the ATC training runs was 45 minutes long. The scenarios used to create the data collection runs were 100 minutes long. As traffic began to build (approximately the $10^{\text {th }}$ minute), participant controllers were given sector briefings and asked to start their participation in the simulation. During the building period, scripted events were limited to routine pilot-to-controller interactions (e.g., climb or descend requests and direct routing requests), and when appropriate, routine pilotto-pilot interactions (e.g., requests for information and coordination of maneuvers). By design, there were no pilot-to-pilot interactions during $\mathrm{CO}$ and CO:CDTI conditions. Table 3 describes traffic scenario characteristics.

Table 3. Traffic Scenario Characteristics

\begin{tabular}{|c|c|c|c|c|}
\hline \multirow{2}{*}{$\begin{array}{c}\text { Experimental } \\
\text { Condition }\end{array}$} & \multicolumn{2}{|c|}{ Traffic Volume } & \multirow{2}{*}{$\begin{array}{l}\text { Duration } \\
\text { (minutes) }\end{array}$} & \multirow[t]{2}{*}{ Purpose } \\
\hline & ATC & CDTI-AL & & \\
\hline CO:CDTI & $\begin{array}{l}\text { Moderate } \\
\text { to High }\end{array}$ & N/A & 45 & $\begin{array}{c}\text { ATC } \\
\text { Training }\end{array}$ \\
\hline SS:Ll & $\begin{array}{l}\text { Moderate } \\
\text { to High }\end{array}$ & N/A & 45 & $\begin{array}{c}\text { ATC } \\
\text { Training }\end{array}$ \\
\hline SS:L2 & $\begin{array}{l}\text { Moderate } \\
\text { to High }\end{array}$ & N/A & 45 & $\begin{array}{c}\text { ATC } \\
\text { Training }\end{array}$ \\
\hline $\mathrm{CO}$ & $\begin{array}{l}\text { Moderate } \\
\text { to High }\end{array}$ & High $^{10}$ & 100 & $\begin{array}{c}\text { Data } \\
\text { Collection }\end{array}$ \\
\hline$\overline{\mathrm{CO}: \mathrm{CDTI}}$ & $\begin{array}{l}\text { Moderate } \\
\text { to High }\end{array}$ & High & 100 & $\begin{array}{c}\text { Data } \\
\text { Collection }\end{array}$ \\
\hline$\overline{\text { SS:LI }}$ & $\begin{array}{l}\text { Moderate } \\
\text { to High }\end{array}$ & High & 100 & $\begin{array}{c}\text { Data } \\
\text { Collection }\end{array}$ \\
\hline SS:L2 & $\begin{array}{l}\text { Moderate } \\
\text { to High }\end{array}$ & High & 100 & $\begin{array}{c}\text { Data } \\
\text { Collection }\end{array}$ \\
\hline
\end{tabular}

All aircraft callsigns and the destination airports for the conflict aircraft were changed.

${ }^{9}$ ATC moderate-10-high traffic volume was emulated as greater than 16 aircraft for sector 21 control, and greater than 13 aircraft for sector 44 control.

${ }^{10} \mathrm{CDTI}-\mathrm{AL}$ high traffic volume was emulated at 15 or greater aircraft visible on the display. 


\subsubsection{Planned Conflicts and NASA 20-Minute Flight Segments}

Conflict detection and resolution is an integral part of air traffic control. To assist in the evaluation of shared-separation operations, planned conflicts between aircraft occurred in each run. All planned conflicts involved two aircraft converging at an acute angle.

ATC training runs had eight planned conflicts between simulated aircraft (the NASA ARC simulator and intruder aircraft did not join these runs as they had independent training). Each of the four data collection runs (CO, CO:CDTI, SS:L1, and SS:L2) had 16 planned conflicts. Eight 2-aircraft conflicts of similar complexity were planned in each sector. In sector 21, the NASA ARC simulator and intruder aircraft pair were involved in three out of the eight planned conflicts. Once a conflict was resolved between them and the aircraft moved out of the sector, the simulators rejoined the run as different aircraft with different call signs. Each of these three "flight segments" for the NASA ARC simulator and intruder aircraft lasted approximately 20 minutes. In sector 44 , WJHTC simulation pilots flew all planned conflict aircraft pairs.

\subsubsection{Pilot Right-of-Way Rules}

During SS:L1 and SS:L2 runs, pilot participants and simulation pilots were instructed to use FAR Part 91 right-of-way rules (when possible) while resolving their own conflicts. Although the right-of-way rules are normally only applicable during visual meteorological conditions, it has been suggested that they may be applied in shared-separation operations to help guide negotiations (RTCA, 1995). All planned conflicts involving simulation pilots were scripted (by the rules) for resolution action and communications. The pilot right-of-way rules were as follows:

- The aircraft on the right had the right-of-way.

- The aircraft being overtaken had the right-of-way.

- Aircraft that were converging head-on each should have altered course to the right.

- During most conflict situations, the aircraft that did not have the right-of-way should have initiated the communication with the aircraft that had the right-of-way.

\subsubsection{Phraseology}

Except for the pilot participants, all simulation pilot phraseology was scripted. The pilot participants were instructed to use the phraseology that they would use based on their current procedures in domestic airspace. The only specific instruction provided to the flight crews regarding phraseology pertained to free flight cancellation. If the flight crew wanted to terminate free flight, they were instructed to state their aircraft identifier (ACID) and specifically indicate to the controller that they wanted to "cancel free flight." flight crews were also told that they could contact any other aircraft, but they were not given any phraseology recommendations for those communications. In addition to the standard phraseology as described in the FAA Order 7110.65 (FAA, 2000), controllers were trained on additional simulation phraseology (see Table 4). 
Table 4. Additional Controller Simulation Phraseology

\begin{tabular}{|l|l|l|}
\hline \multicolumn{1}{|c|}{ Action } & \multicolumn{1}{|c|}{ Phraseology } & Condition \\
\hline $\begin{array}{l}\text { Cancellation of Free } \\
\text { Flight }\end{array}$ & $\begin{array}{l}\text { Controller - "ACID (and ACID), free flight } \\
\text { canceled" and issue the appropriate control } \\
\text { action. }\end{array}$ & SS:L1 \\
\hline $\begin{array}{l}\text { Resumption of Free } \\
\text { Flight }\end{array}$ & $\begin{array}{l}\text { Controller - "ACID (and ACID), resume free } \\
\text { flight." }\end{array}$ & SS:L1 \\
\hline $\begin{array}{l}\text { Acknowledge Pilot } \\
\text { intentions }\end{array}$ & $\begin{array}{l}\text { Pilot - informs controller of an intended } \\
\text { maneuver. } \\
\text { Controller - "ACID, roger" }\end{array}$ & SS:L1 \\
\hline $\begin{array}{l}\text { Aircraft coordination } \\
\text { for RED URET alerts }\end{array}$ & $\begin{array}{l}\text { Controller - "ACID, traffic alert with ACID at } \\
\text { (altitude) at (time), advise intentions" }\end{array}$ & $\begin{array}{l}\text { SS:L1 and } \\
\text { SS:L2 }\end{array}$ \\
\hline $\begin{array}{l}\text { Sector coordination for } \\
\text { RED URET alerts }\end{array}$ & $\begin{array}{l}\text { Controller Sector 1 - "Traffic alert ACID" } \\
\text { Controller Sector 2 - "Go ahead" } \\
\text { Controller Sector 1 - "ACID with } A C I D \text { at } \\
\text { altitude at time" }\end{array}$ & $\begin{array}{l}\text { SS:L1 and } \\
\text { SS:L2 }\end{array}$ \\
\hline
\end{tabular}

\subsubsection{Frequencies}

In addition to land lines and sector frequencies, the experiment emulated an air $\leftrightarrow$ air frequency. The air $\leftrightarrow$ air frequency was provided to avoid frequency congestion problems and was only available during SS:L1 and SS:L2 conditions. On the air $\leftrightarrow$ air frequency, the pilots were able to communicate among themselves and negotiate resolution strategies. Controllers were able to selectively monitor the frequency as desired but were not permitted to transmit on the frequency. Table 5 provides the frequencies used in this experiment. 
Table 5. Sector Frequencies

\begin{tabular}{|c|l|}
\hline \multicolumn{1}{|c|}{ Type } & \multicolumn{1}{c|}{ Frequency } \\
\hline SECTOR 21 & 132.42 \\
\hline SECTOR 44 & 124.92 \\
\hline AIR $\leftrightarrow$ AIR & 122.75 \\
\hline SECTOR 78 & 123.45 \\
\hline
\end{tabular}

\subsection{Simulation Constraints and Assumptions}

Though this particular experiment emulated aspects of the operational environment at high fidelity levels, all simulation studies presume some limitations and assumptions. Therefore, the results of this work and all similar experimental research should be interpreted with caution. The following describe recognized limitations and constraints of AGIE.

- This study had a limited number of participants and therefore limited power for the use of inferential statistics. The experiment originally called for five groups of controllers $(20$ individuals) and pilots ( 10 individuals). Due to events beyond the experiment team's control, only three groups of each were obtained" .

- The original design of the simulation called for five unique traffic scenarios: one for ATC training runs and four for data collection runs. Due to time constraints and the unexpected complexity of the design process, only two data collection traffic scenarios were created. For those two scenarios, all aircraft callsigns and the destination airports of conflict aircraft were changed to create four unique data collection runs. In the exit forms, participants reported that the runs were familiar.

- The aircraft simulator used for this study was a Boeing 747-400. This aircraft is typically used for long-haul oceanic flying. The flight crews used were those qualified on this aircraft type to insure minimal training. The use of commercial pilots who typically flew long oceanic routes may have affected the flight crew results. In particular, fuel considerations are very important to oceanic pilots due to the length of their flights. Thus, some pilots expressed concern over the use of altitude changes while maneuvering due to concern for additional fuel consumption and aircraft weight. Although this study was not conducted in the oceanic environment, oceanic flight crew participants may have constrained some considerations for aircraft maneuvers.

"The fourth controller group (OS) data were used only for comment analyses. Data from the fourth pilot group was not included because one pilot had previously participated in the study. 
- Due to relatively short flight segments (20 minutes), most air-side efficiency measures were not possible to analyze.

- Some of the aircraft entered the simulation too close to the sector boundary. This was a technical limitation in the laboratory.

- The $I^{2} F$ laboratory does not have VSCS equipment. The system emulated had different capabilities and was apparently somewhat cumbersome.

- Because the NASA ARC simulator flights involved the real pilots and the remaining aircraft communications were provided by simulation pilots, the controllers were able to distinguish the pilot participants from most of the simulation pilots due to phraseology, style of communications, and clearer frequencies from the simulation pilot laboratories.

- The DSR version BABO3 used in the simulation was one version behind the operational field (BABO4) at the time of the study. Differences were minimal.

- There was an unidentified anomaly regarding the start of two conflicts in each run of CO:CDTI and SS:L2. URET would not automatically recognize the existence of the involved aircraft. Manual flight plan activation for these aircraft was included in the simulation as a successful and unobtrusive procedural fix.

\subsection{Procedures}

AGIE was conducted concurrently at the WJHTC on the east coast and NASA ARC on the west coast. Therefore, simulation procedures were conducted simultaneously at both locations. The following sections describe all activities in detail.

\subsubsection{WJHTC Pre-Simulation Activities}

In the weeks prior to simulation, WJHTC simulation pilots and ghost sector controllers were rigorously trained to assure operationally consistent, accurate, and timely responses to controller instructions and requests. Lectures on the following topics were performed:

- study objectives,

- study methodology,

- airspace structure,

- air traffic characteristics,

- aircraft equipage,

- controller procedures, and

- anticipated controller actions during shared-separation operations.

Additionally, the WJHTC simulation pilots and ghost sector controllers exercised all experimental conditions over a 2-week shakedown period. Particular emphasis was placed on reacting to unexpected pilots actions and timely execution of scripted events. 


\subsubsection{WJHTC Simulation Activities}

Each group of controllers and the EOs participated for a 3-day simulation period (from 12 to 8 p.m. EST). A daily schedule for controller participants is provided in Appendix A.

On the first day, they were provided an experiment briefing. Following the initial briefing, controllers were assigned to a sector (21 or 44) and position (R-side or D-side), which remained constant throughout the experiment. The EOs were also assigned to a sector. Laboratory familiarization and hands-on training followed.

On the second day, controllers and EOs were provided additional hands-on training. Following the completion of all training, data collection runs started. At the beginning of each run, participants were again briefed on the procedures of the particular condition they were about to experience. They were also provided with an aid chart on their control position describing the key procedures for the run. During each run, the EOs watched sector operations and recorded interesting and critical events.

Data collection runs continued through the third day. At the end of all data collection runs, the experiment team held a semi-structured group debriefing session. The purpose of this debriefing was to provide an opportunity to share information that was not captured in the forms.

Participation in this study was strictly voluntary, and the privacy of all participants was and will be protected. Strict adherence to all federal, union, and ethical guidelines was maintained throughout the study.

\subsubsection{Controller Briefing}

Members of the experiment team briefed the participants in a classroom setting prior to entering the laboratory area. The participants were encouraged to ask questions. The participants were also provided with the briefing materials contained in Appendix B.

The briefing covered the following topics:

- Human Research Minimal Risk Consent Document,

- Participant's role in the study,

- Study objectives,

- Study methodology,

- Airspace structure,

- Aircraft equipage and procedures,

- Air traffic characteristics,

- Laboratory equipment and configuration, and

- Rules and procedures. 
Following the briefing, the participants were requested to complete the Background Information Form (Appendix C) and the Human Research Minimal Risk Consent Document (Appendix D).

\subsubsection{Laboratory Familiarization}

Although the $I^{2} \mathrm{~F}$ was configured to replicate $\mathrm{ZME}$ sectors 44 and 21 with high fidelity, differences between the field and the laboratory configuration existed. All differences were briefed in detail and instructions on equipment usage were provided. Equipment training and laboratory familiarization lasted approximately 2 hours.

\subsubsection{Controller Training}

Three, 45-minute training runs were provided to allow participants to gain experience with shared-separation operations and provide additional practice with the laboratory equipment ${ }^{12}$. Members of the experiment team and EOs were available throughout training to answer questions. A 15-minute classroom group discussion followed each training run.

\subsubsection{Experimental Condition Run Order}

Following the training runs, data collection activities started. All participant groups (controller and pilot) participated in all four runs. The order of condition presentation was counter-balanced across the four data collection groups. Table 6 depicts the order of condition presentation.

Table 6. Run Order by Group

\begin{tabular}{|l|l|l|l|l|}
\hline & \multicolumn{1}{|c|}{ Group 1 } & \multicolumn{1}{|c|}{ Group 2 } & \multicolumn{1}{|c|}{ Group 3 } & \multicolumn{1}{|c|}{ Group 4 } \\
\hline Run 1 & CO & CO:CDTI & SS:L2 & SS:L1 \\
\hline Run 2 & SS:L2 & SS:L1 & CO & CO:CDTI \\
\hline Run 3 & CO:CDTI & CO & SS:L1 & SS:L2 \\
\hline Run 4 & SS:L1 & SS:L2 & CO:CDTI & CO \\
\hline
\end{tabular}

\subsubsection{NASA ARC Pre-Simulation Activities}

In the weeks prior to simulation, the intruder simulation pilot was trained to assure operationally consistent, accurate, and timely responses to controller instructions and requests. In addition, the simulation pilot was instructed on how to provide operationally realistic air $\leftrightarrow$ air communications in a shared-separation context. The following topics were discussed:

\footnotetext{
${ }^{12}$ Due to independent training sessions, the NASA ARC simulator and intruder aircraft did not participate in these training scenarios.
} 
- Study objectives,

- Study methodology,

- Airspace structure,

- Air traffic characteristics,

- Aircraft equipage,

- Controller procedures,

- Anticipated controller actions during shared-separation operations, and

- Anticipated flight crew comments and maneuvering during shared-separation operations.

The intruder simulation pilot participated in trial runs of all conditions prior to the beginning of data collection. The trials included air $\leftrightarrow$ ground communications, air $\leftrightarrow$ air communications, and maneuvering of the simulator aircraft.

\subsubsection{NASA ARC Simulation Activities}

Each flight crew participated in the study for two 8-hour days (from 9 to 5 p.m. EST). A daily schedule for pilot participants is provided in Appendix E.

On the morning of the first day, the pilot participants were given an experiment briefing and training on the tasks they would perform during the experiment. All pilot participants were qualified on the Boeing 747-400 aircraft type; therefore, no training or familiarization with the simulator was required, with the exception of the new flight deck tools provided for sharedseparation.

Data collection runs began on the afternoon of the first day. Each condition (CO, CO:CDTI, SS:L1, and SS:L2) had three flight segments. At the end of the three flight segments representing a condition, a form was given to address questions regarding tasks and workload during that condition. At the end of all data collection, pilot participants completed two more forms and were debriefed to obtain all their feedback relative to the goals and tasks of the study. See Appendix F for these pilot forms.

\subsubsection{Flight Crew Briefing}

Flight crews participated in a 90-minute briefing covering the general goals of the free flight concept and this study. The briefing emphasized the potential for increased operational flexibility and efficiency with free flight. Topics in the briefing also included

- the Human Research Minimal Risk Consent Document;

- roles of pilots and controllers in this study;

- new display features, alerting logic, and underlying technology assumptions;

- maneuvering and communication procedures, including pilot right-of-way rules;

- rules governing free flight for each applicable condition; 
- general description of flight information (e.g., destination and path); and

- safety briefing for NASA ARC simulator.

Following the briefing, the participants were requested to complete the Human Research Minimal Risk Consent Document contained in Appendix D.

\subsubsection{Flight Crew Training}

Following the briefing, pilots participated in approximately 90 minutes of training in the NASA ARC simulator. The flight crew training runs were different from the actual experimental runs, but they exposed the participants to the different conditions and procedures under which the crews were expected to operate. The crews were provided with a sense of the timing parameters associated with the alerting logic and had an opportunity to practice the verbal procedures used in air $\leftrightarrow$ air and air $\leftrightarrow$ ground communication. Questions by the flight crew participants were encouraged during both the briefing and training sessions.

\subsubsection{Experimental Condition Run Order}

All participant groups (controller and pilot) participated in all four runs. Each flight crew flew three 20-miniute fight segments per run. The order of condition presentation was random and counter-balanced across the four data collection groups.

\subsection{Data Collection}

Subjective and objective data were collected throughout the study from the ground-side (participant controllers, EOs, and the ATC environment), and the air-side (pilot participants and the flight deck).

\subsubsection{Ground-Side Subjective Data}

Subjective data were collected from controllers and EOs via forms (Appendix C), interval workload ratings, and debriefing sessions.

\subsubsection{Form Data}

Participant controllers and EOs completed the Background Information Form immediately after the initial briefing session at the WJHTC. The background forms solicited information related to professional experience and other relevant information.

EOs also completed the During-the-Run Form throughout the simulation. Using this form, the EOs recorded critical events, free flight cancellations, controller actions, and observations related to the impact of conflicts and shared-separation operations.

At the end of each run, both the participant controllers and EOs completed post-run forms. The Controller Post-Run Form solicited information regarding the traffic, simulation environment, workload ratings, and impact of conflicts and shared-separation operations. The EO Post-Run Form solicited information regarding the overall workload and impact of conflicts and sharedseparation operations. 
At the end of all runs, the participant controllers completed an Exit Form. This form elicited information regarding simulation fidelity, adequacy of training for simulation, automation needs, and the effects of shared-separations operations.

\subsubsection{Interval Workload Data}

During each run, the participant controllers rated their instantaneous workload (combined cognitive and physical) on a $1-$ to- 5 scale ( $1=$ very low, $3=$ moderate, and $5=$ very high), at 5-minute intervals, using WAKs. In the few instances when the laptop and/or WAK did not function properly, researchers supplied paper forms to participant controllers to record workload ratings.

The following instructions were given to participant controllers regarding interval workload ratings:

- The WAK will illuminate and sound a small beep at every 5-minute interval. At that time, you are requested to press a key corresponding to your instantaneous workload level.

- When reporting your workload rating, please consider both cognitive and physical workload.

- The workload rating scale was as follows:

\begin{tabular}{|c|c|c|c|c|}
\hline 1 & 2 & 3 & 4 & 5 \\
\hline Very Low & & Moderate & & Very High \\
\hline
\end{tabular}

The operational meaning of the rating scale was explained as follows:

\section{Rating Operational Meaning}

1 Your workload is very low and you can complete all tasks.

2 Your workload is rather low and there is little chance for an error in your tasks.

3 Your workload is moderate and there is an increasing chance of error in your tasks.

4 Your workload is rather high and there is some chance for an error in your tasks.

5 Your workload is very high and you may have to leave some tasks incomplete. 


\subsubsection{Conflict Detection and Resolution Measures}

The participant controllers were asked to provide different points of conflict detection and resolution as follows:

Point A - Conflict Detection Point: The first point was collected for all four experimental conditions and was recorded at the point when controllers first detected a potential conflict between aircraft with reasonable certainty.

Point B - Conflict Resolution Point under Current Operations: The second point was collected only for SS:L1 and SS:L2. The second point was recorded at the point when controllers would have taken action to resolve a potential conflict under current operating rules (as described in 7110.65 [FAA, 2000]). Controllers were asked to assume that not all flight crews have CDTI-AL.

Point C - Conflict Resolution Point under SS:L2 Operations: The third point was collected only for SS:L2. The third point was recorded when controllers would have taken action to resolve a potential conflict under SS:L2 conditions where all flight crews have CDTI-AL and are responsible for separation.

The EOs recorded these points for each sector and noted the time on the Observer During-theRun-Form. For Point A, the EOs also noted whether the controller used URET.

Results and subsequent analyses of these data were intended to address controller situation awareness. However, there were problems with the automated tool used to collect these data resulting in unacceptable levels of accuracy. Therefore, results and discussion about these data are not included in the report.

\subsubsection{Debriefing Sessions}

Semi-structured, debriefing sessions were conducted with each controller group and the EOs. The controllers and EOs were given the opportunity to provide any additional information about their experiences in the simulation, their thoughts about the concepts, procedures and tools investigated in this project, and to have the researchers answer any remaining questions. All forms provided blank spaces for participants to provide open-ended, descriptive information and comments.

The debriefings were recorded on audiocassettes. Table 7 summarizes subjective data that were collected during the simulation. 
Table 7. Ground-Side Subjective Data

\begin{tabular}{|l|l|l|l|}
\hline \multicolumn{1}{|c|}{ Instrument } & \multicolumn{1}{|c|}{ Users } & \multicolumn{1}{c|}{ Completed } & \multicolumn{1}{c|}{ Objective } \\
\hline Background Form & $\begin{array}{l}\text { Controllers } \\
\text { EOs }\end{array}$ & $\begin{array}{l}\text { Once before } \\
\text { first training run }\end{array}$ & $\begin{array}{l}\text { Gather demographic } \\
\text { information. }\end{array}$ \\
\hline $\begin{array}{l}\text { During-the-Run } \\
\text { Form }\end{array}$ & EOs & During each run & $\begin{array}{l}\text { Record critical and interesting } \\
\text { events. }\end{array}$ \\
\hline $\begin{array}{l}\text { Interval Workload } \\
\text { Ratings }\end{array}$ & Controllers & $\begin{array}{l}\text { Every } 5 \text { minutes } \\
\text { during each run }\end{array}$ & $\begin{array}{l}\text { Electronically record controller } \\
\text { workload ratings using WAK. }\end{array}$ \\
\hline $\begin{array}{l}\text { Point A - Conflict } \\
\text { Detection }\end{array}$ & Controllers & During each run & $\begin{array}{l}\text { Record on the During-the-Run } \\
\text { Observer Form. }\end{array}$ \\
\hline $\begin{array}{l}\text { Point B - Conflict } \\
\text { Resolution }\end{array}$ & Controllers & $\begin{array}{l}\text { During SS:L1 } \\
\text { and SS:L2 runs }\end{array}$ & $\begin{array}{l}\text { Record on the During-the-Run } \\
\text { Observer Form. }\end{array}$ \\
\hline $\begin{array}{l}\text { Point C - Conflict } \\
\text { Resolution }\end{array}$ & Controllers & $\begin{array}{l}\text { During SS:L2 } \\
\text { runs }\end{array}$ & $\begin{array}{l}\text { Record on the During-the-Run } \\
\text { Observer Form. }\end{array}$ \\
\hline Post-Run Form & Controllers & After each run & $\begin{array}{l}\text { Elicit controller comments and } \\
\text { ratings related to the conflict } \\
\text { situations, communications, } \\
\text { shared-separation, scenario } \\
\text { information, workload, } \\
\text { situation awareness, and so on. }\end{array}$ \\
\hline Exit Form & Controllers & End of all runs & $\begin{array}{l}\text { Gather information regarding } \\
\text { impact of shared-separation } \\
\text { and conflicts on workload, } \\
\text { automation needs, simulation } \\
\text { training adequacy and fidelity. }\end{array}$ \\
\hline De-briefing & EOs & End of all runs & $\begin{array}{l}\text { Collective discussions of } \\
\text { shared-separations and gather } \\
\text { ground-side information that } \\
\text { was not previously acquired. }\end{array}$ \\
\hline on. & $\begin{array}{l}\text { Record EO observations } \\
\text { related to conflicts, } \\
\text { communications, shared- } \\
\text { separation, workload, and so }\end{array}$ \\
\hline
\end{tabular}




\subsubsection{Ground-Side Objective Data}

Objective data related to URET alerts and trial plans, voice communication data, Host data, and audio and video data were collected.

- URET Alerts and Trial Plans

The number and duration of URET-reported red and yellow alerts were extracted from the URET data logs. The frequency of URET trial plans was also extracted from the URET log.

- Push-to-Talk Transmission Data

The number and duration of ground $\rightarrow$ air and land line push-to-talk transmissions (PTTs) were recorded. A ground $\rightarrow$ air PTT is defined as a verbal message from the controller to the pilot, the duration of which is measured from the onset to the end of a key press.

- Host Data

The number of aircraft at every five-minute interval, the total number of aircraft in each run, separation violations, MSD data, and conflict alerts reported by the Host were extracted from SAR tapes.

- Audio and Video Recordings

Each run was video and audio recorded to capture the interaction between controllers. The purpose was to gather supplemental data to assess workload levels and to substantiate other subjective and objective data. Audio recordings captured the ambient conversations between controllers and all simulation frequencies. Video recordings captured general views of the sectors and the URET displays. For each run, the video and audio were recorded onto four tapes (two tapes per sector). Table 8 summarizes the audio and video recordings.

Table 8. ATC Audio and Video Recording

\begin{tabular}{|c|c|c|c|}
\hline Tape & Video View & $\begin{array}{c}\text { Left Audio } \\
\text { Communications }\end{array}$ & $\begin{array}{c}\text { Right Audio } \\
\text { Communications }\end{array}$ \\
\hline 1 & S44 Overview & $\begin{array}{c}\text { S44 air } \leftrightarrow \text { ground, } \\
\text { R-side/D-side ambient }\end{array}$ & $\begin{array}{c}\text { land line, } \\
\text { R-side/D-side } \\
\text { ambient }\end{array}$ \\
\hline 2 & S21 Overview & S21 air $\leftrightarrow$ ground, land line & $\begin{array}{c}\text { S21 air } \leftrightarrow \text { ground, } \\
\text { R-side/D-side } \\
\text { ambient }\end{array}$ \\
\hline 3 & $\begin{array}{c}\text { S44 } \\
\text { URET Display }\end{array}$ & $\begin{array}{c}\text { S44 air } \leftrightarrow \text { ground, land line } \\
\text { R-side/D-side ambient }\end{array}$ & $\begin{array}{c}\text { Ghost Controller } \\
\text { air } \leftrightarrow \text { ground }\end{array}$ \\
\hline 4 & $\begin{array}{c}\text { S21 } \\
\text { URET Display }\end{array}$ & $\begin{array}{c}\text { All Sector 21 audio channels, } \\
\text { R-side/D-side ambient }\end{array}$ & air $\leftrightarrow$ air \\
\hline
\end{tabular}


Table 9 lists objective data that were collected during each run during.

Table 9. Ground-Side Objective Data Summary

\begin{tabular}{|l|l|}
\hline \multicolumn{1}{|c|}{ Data } & \multicolumn{1}{c|}{ Source of Data } \\
\hline Number and duration of ground $\rightarrow$ air PTT & PTT recordings \\
\hline Number and duration of land line PTT & PTT recordings \\
\hline Peak traffic count & SAR tapes \\
\hline Traffic density & SAR tapes \\
\hline $\begin{array}{l}\text { MSD of all planned conflicts (except NASA } \\
\text { ARC simulator conflicts) }\end{array}$ & SAR tapes \\
\hline Number of URET trial flight plans & URET log \\
\hline $\begin{array}{l}\text { Number and duration of URET red and yellow } \\
\text { conflict alerts }\end{array}$ & URET log \\
\hline Number and duration of separation violations & SAR tapes \\
\hline $\begin{array}{l}\text { Number of Free Flight cancellations by } \\
\text { controllers }\end{array}$ & Observer and controller forms \\
\hline $\begin{array}{l}\text { Number of Free Flight cancellation requests by } \\
\text { pilots }\end{array}$ & Observer and controller forms \\
\hline
\end{tabular}

\subsubsection{Air-Side Subjective Data}

Subjective data were collected from the individual pilot participants primarily through post-run and exit forms. Additional data were collected during a debriefing session and workload data were collected following each flight segment.

\subsubsection{Form Data}

Following each flight segment, each pilot participant provided a rating of overall workload for the 20-minute flight segment. Workload ratings were collected using a 5-point Likert scale.

Following each run, which consisted of three 20 -min flight segments, the pilot participants completed a Post-Run Form (Appendix F). This form assessed various aspects of pilots' experiences with the procedures and/or tools used during that run, such as ratings of workload, situation awareness, safety, and effectiveness of the new flight deck tools. 
Upon completion of the experiment, pilot participants were given an Exit Form (Appendix F). This form collected pilots' demographic information, as well as assessing simulation fidelity and integrity and the pilots' overall evaluation of tools and procedures used in the study.

\subsubsection{Debriefing Sessions}

Debriefing sessions were conducted with each flight crew. The flight crews were given the opportunity to provide any additional information about their experiences in the simulation, their thoughts about the concepts, procedures and tools investigated in this project, and to have the researchers answer any remaining questions. All forms provided blank spaces for participants to provide open-ended, descriptive information and comments. Table 10 summarizes subjective data that were collected during the simulation.

Table 10. Flight Crew Subjective Data

\begin{tabular}{|l|l|l|}
\hline \multicolumn{1}{|c|}{ Instrument } & \multicolumn{1}{|c|}{ Completed } & \multicolumn{1}{c|}{ Objective } \\
\hline $\begin{array}{l}\text { Post-Flight Workload } \\
\text { Rating }\end{array}$ & $\begin{array}{l}\text { After each flight } \\
\text { segment }\end{array}$ & $\begin{array}{l}\text { Assess pilot participants' ratings of } \\
\text { overall workload across the preceding } \\
\text { 20-min flight segment. }\end{array}$ \\
\hline Post-Run Form & After each run & $\begin{array}{l}\text { Assess pilot participants' ratings of the } \\
\text { impact of the tools and/or procedures on } \\
\text { safety, information requirements, } \\
\text { workload, situation awareness, and so } \\
\text { on used during that run. }\end{array}$ \\
\hline Exit Form & End of all runs & $\begin{array}{l}\text { Gather information regarding impact of } \\
\text { shared-separation and aircraft conflicts } \\
\text { on workload, automation needs, } \\
\text { simulation training adequacy, and } \\
\text { fidelity across entire experiment. }\end{array}$ \\
\hline Debriefing & End of all runs & $\begin{array}{l}\text { Gather any additional information } \\
\text { concerning the pilots' experiences in the } \\
\text { simulation and to discuss the concepts, } \\
\text { procedures, and tools under study. }\end{array}$ \\
\hline
\end{tabular}

\subsubsection{Air-Side Objective Data}

Objective data were collected during each flight segment from the NASA ARC simulator computer systems, including data on use of various aircraft flight systems, use of flight deck display controls, and communication equipment. Video and audio recordings of flight crew interactions on the flight deck were also collected. 


\subsubsection{NASA ARC Simulator Data}

The MSD, number of separation violations, free flight cancellation distances, maneuver type and timing, navigation display information, and CDTI-AL parameters relevant to the NASA ARC simulator were collected.

\subsubsection{Audio and Video Recordings}

Each flight segment was audio and video recorded to capture the interactions between the pilot participants and their use of the aircraft systems as well as the communication exchanges with controllers and all simulation pilots. Video and audio were recorded onto two tapes. Table 11 depicts the recording descriptions. These recordings served as source data for several of the variables described in Table 12.

Table 11. Air-Side Audio and Video Recordings

\begin{tabular}{|c|l|l|l|}
\hline Tape & \multicolumn{1}{|c|}{ Video View } & \multicolumn{1}{|c|}{$\begin{array}{c}\text { Left Audio } \\
\text { Communications }\end{array}$} & \multicolumn{1}{|c|}{$\begin{array}{c}\text { Right Audio } \\
\text { Communications }\end{array}$} \\
\hline 1 & $\begin{array}{l}\text { Quadrant A: Captain's } \\
\text { Primary Flight Display }\end{array}$ & $\begin{array}{l}\text { ambient flight deck } \\
\text { communication }\end{array}$ & $\begin{array}{l}\text { selected frequencies, } \\
\text { (e.g. air } \leftrightarrow \text { ground, } \\
\text { air } \leftrightarrow \text { air) }\end{array}$ \\
\cline { 2 - 4 } & $\begin{array}{l}\text { Quadrant B: Captain's } \\
\text { Navigation Display }\end{array}$ & $\begin{array}{l}\text { ambient flight deck } \\
\text { communication }\end{array}$ & $\begin{array}{l}\text { selected frequencies, } \\
\text { (e.g. air } \leftrightarrow \text { ground, } \\
\text { air } \leftrightarrow \text { air) }\end{array}$ \\
\cline { 2 - 5 } \\
$\begin{array}{l}\text { Flight Management } \\
\text { Funputer (FMC)/Multi- } \\
\text { Unit (MCDU) }\end{array}$ & $\begin{array}{l}\text { ambient flight deck } \\
\text { communication }\end{array}$ & $\begin{array}{l}\text { selected frequencies, } \\
\text { (e.g. air } \leftrightarrow \text { ground, } \\
\text { air } \leftrightarrow \text { air) }\end{array}$ \\
\cline { 2 - 5 } & $\begin{array}{l}\text { Quadrant D: First Officer's } \\
\text { FMC/MCDU }\end{array}$ & $\begin{array}{l}\text { ambient flight deck } \\
\text { communication }\end{array}$ & $\begin{array}{l}\text { selected frequencies, } \\
\text { (e.g. air } \leftrightarrow \text { ground, } \\
\text { air } \leftrightarrow \text { air) }\end{array}$ \\
\hline 2 & $\begin{array}{l}\text { Captain's Navigation } \\
\text { Display }\end{array}$ & $\begin{array}{l}\text { ambient flight deck } \\
\text { communication }\end{array}$ & $\begin{array}{l}\text { selected frequencies, } \\
\text { air } \leftrightarrow \text { air } \leftrightarrow \text { ground, }\end{array}$ \\
\hline
\end{tabular}


Table 12. Air-Side Objective Data Summary

\begin{tabular}{|l|l|}
\hline \multicolumn{1}{|c|}{ Data } & \multicolumn{1}{|c|}{ Source of Data } \\
\hline $\begin{array}{l}\text { Number and duration of air } \leftrightarrow \text { ground communications } \\
\text { involving NASA ARC simulator }\end{array}$ & Video/audio recordings \\
\hline $\begin{array}{l}\text { Number of air } \leftrightarrow \text { air communications involving NASA } \\
\text { ARC simulator }\end{array}$ & Video/audio recordings \\
\hline MSD for all conflicts involving NASA ARC simulator & NASA ARC simulator output data \\
\hline $\begin{array}{l}\text { Number of separation violations for all conflicts } \\
\text { involving NASA ARC simulator }\end{array}$ & NASA ARC simulator output data \\
\hline $\begin{array}{l}\text { Number of free flight cancellations by controller on } \\
\text { conflicts with NASA ARC simulator }\end{array}$ & Video/audio recordings \\
\hline $\begin{array}{l}\text { Number of free flight cancellation requests by pilot } \\
\text { participants }\end{array}$ & Video/audio recordings \\
\hline $\begin{array}{l}\text { Distance between NASA ARC simulator and intruder } \\
\text { aircraft at time of free flight cancellation }\end{array}$ & NASA ARC simulator output data \\
\hline $\begin{array}{l}\text { Type of maneuvers made by NASA ARC simulator } \\
\text { and intruder aircraft }\end{array}$ & $\begin{array}{l}\text { NASA ARC simulator output data and } \\
\text { video recordings }\end{array}$ \\
\hline $\begin{array}{l}\text { Type of maneuvers issued by controllers to NASA } \\
\text { ARC simulator and intruder aircraft }\end{array}$ & $\begin{array}{l}\text { NASA ARC simulator output data and } \\
\text { video recordings }\end{array}$ \\
\hline $\begin{array}{l}\text { Timing of first maneuver made by NASA ARC } \\
\text { simulator and/or intruder aircraft for conflict } \\
\text { resolution }\end{array}$ & $\begin{array}{l}\text { NASA ARC simulator output data and } \\
\text { video recordings }\end{array}$ \\
\hline $\begin{array}{l}\text { Frequency and timing of CDTI-AL alerts as displayed } \\
\text { on the navigation display of NASA ARC simulator }\end{array}$ & $\begin{array}{l}\text { NASA ARC simulator output data and } \\
\text { video recordings }\end{array}$ \\
\hline $\begin{array}{l}\text { Conflict detection times for pilot participants for } \\
\text { conflicts involving NASA ARC simulator }\end{array}$ & Video/audio recordings \\
\hline $\begin{array}{l}\text { Time spent at each map range level on navigation } \\
\text { display of NASA ARC simulator }\end{array}$ & NASA ARC simulator output data \\
\hline ind
\end{tabular}




\section{RESULTS}

Within each sub-section, the order of data results corresponds to the three primary objectives of the study.

Due to the exploratory nature of the research and small sample sizes, inspection of the mean and \pm 1 standard error of the mean (SEM) was used as the primary method to analyze the data. In general, if the \pm 1 SEM bars overlapped, then the two means were considered the same. If they did not overlap, then the means were reported as appearing different. The Analysis of Variance (ANOVA) statistical method was sometimes used to provide additional insight for future areas of research, particularly for the ground-side data. In addition, the researchers recognized that the rating scales used in this study reflect ordinal data that typically warrants analysis using descriptive statistics such as median and range. However, in order to provide comparisons to earlier studies and make the results more understandable to a broad audience, more popular measures such as mean and standard deviation $(S D)$ were reported.

\subsection{Ground-Side Results}

Most of the ground-side data are summarized by the four conditions (CO, CO:CDTI, SS:L1, and SS:L2) of the study. Means (M) and either SD or \pm 1 SEM was computed for measures across the 12 controllers who participated. Some measures were also summarized by controller position (R-side, D-side) where appropriate. Most of the ground-side data were also analyzed using ANOVA, and the results are reported in Appendix G. However, due to the limited number of observations in this experimental design, the results should be interpreted with caution. This study is a preliminary investigation, and the results should not be generalized or accepted as conclusive.

\subsubsection{Operational Issues that Affect Shared-Separation Operations}

\subsubsection{Controller Ratings for the Amount of Time Available to Assure Safe Aircraft Separation} and Complete Required Coordination

Figure 11 shows mean controller ratings ( \pm 1 SEM) for time available to assure safe aircraft separation and complete required coordination. In general, controllers rated the amount of time available for both tasks as adequate. Controllers reported slightly more time available to assure safe aircraft separation in CO:CDTI. There were no differences between the four conditions for the time available to complete required coordination. The results of the ANOVAs are reported in Appendix G, Section G-1. 


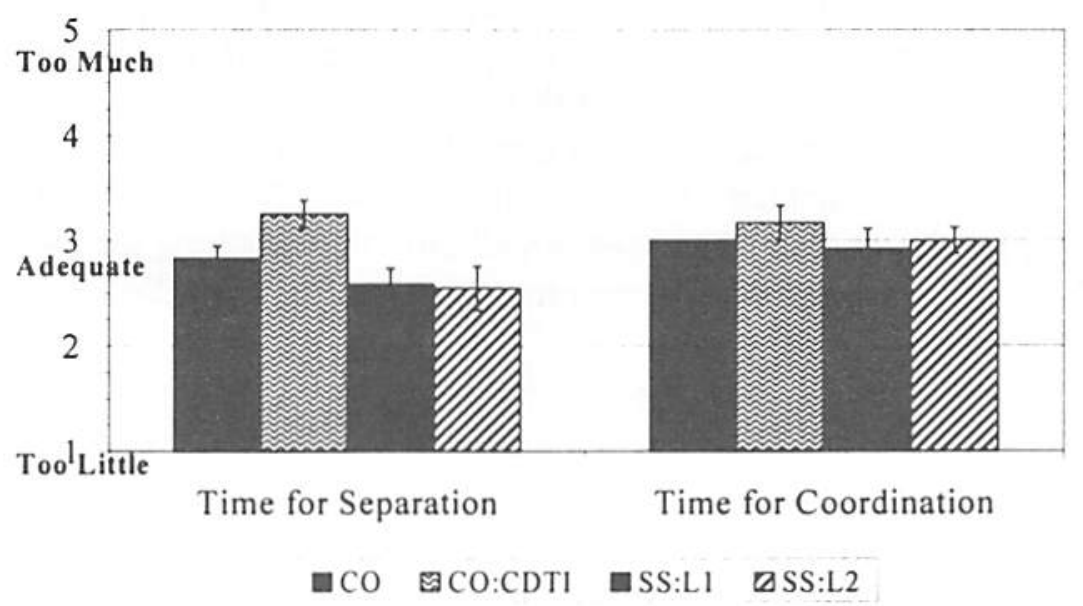

Figure 11. Controller mean ratings for time available for separation and coordination.

\subsubsection{Controller Ratings for the Level of Safety for Procedures Compared to Current} Operations

Figure 12 shows mean controller ratings $( \pm 1$ SEM) of the level of safety for procedures compared to current operations. Controllers rated the level of safety for CO and CO:CDTI as unchanged from current operations. However, controllers rated the level of safety for SS:L1 and SS:L2 as compromised. The results of the ANOVAs are reported in Appendix G, Section G-2.

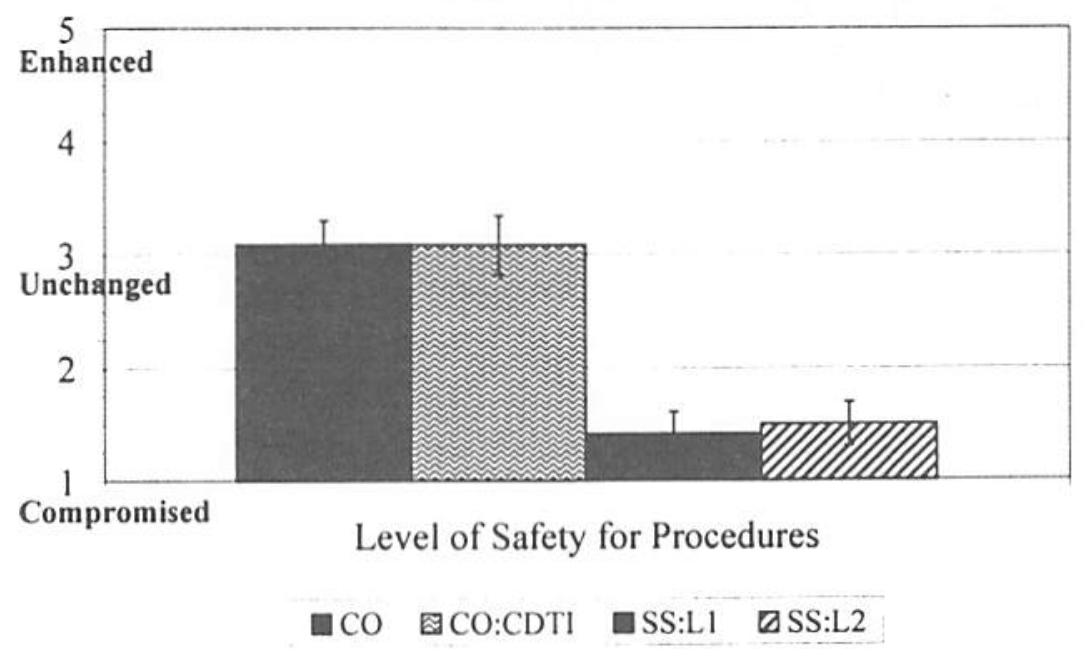

Figure 12. Controller mean ratings of level of safety for procedures. 


\subsubsection{URET Conflict Alerts}

Figure 13 shows mean frequencies (number per run) of URET red and yellow alerts $( \pm 1 \text { SEM })^{13}$. A URET alert for an aircraft pair was only counted once and at the highest level of alert. For example, if an aircraft pair had a yellow alert that progressed to a red alert (without interruption), then the alert was only counted once, as a red alert. However, if an alert on an aircraft pair was terminated and then later reestablished, it was counted again. There were slightly more red alerts than yellow alerts for all conditions, and only small differences among the four conditions for both alerts. The results of the ANOVAs are reported in Appendix G, Section G-3A.

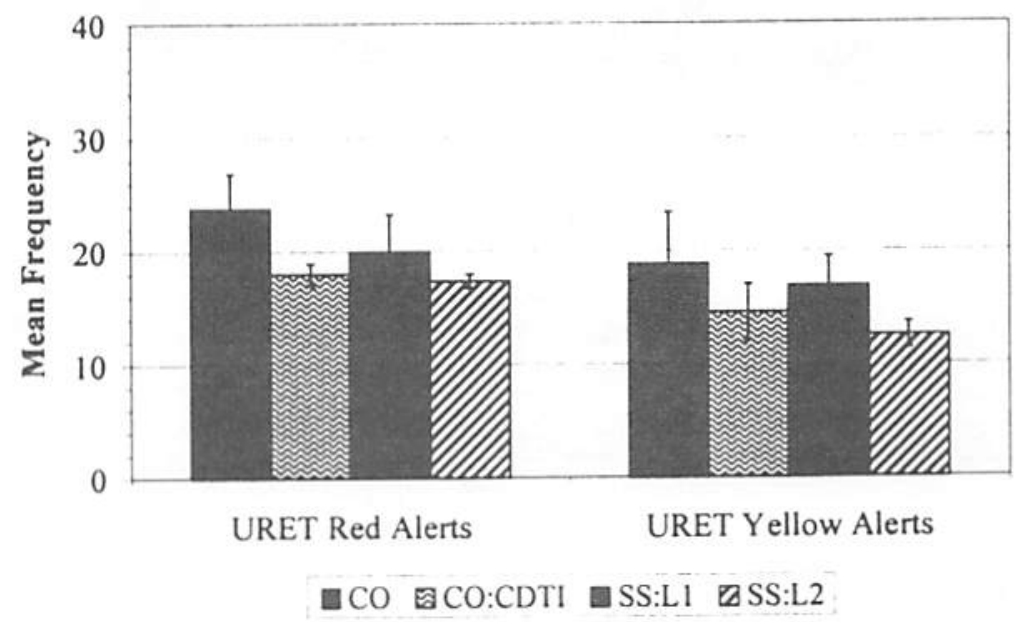

Figure 13. Mean frequency of URET conflict alerts.

Figure 14 shows the mean duration per alert $( \pm 1$ SEM) for URET red and yellow alerts. In general, the duration of red and yellow alerts were longer in SS:L2. The results of the ANOVAs are reported in Appendix G, Section G-3B.

\footnotetext{
${ }^{13}$ By design, there were very few ascending or descending aircraft in the scenarios, therefore, there were very few muted red or yellow alerts. In addition, these alerts were not a primary focus of the study. For these reasons, muted alerts were not analyzed. There were no blue alerts in the study because there was no SUA in the sectors emulated.
} 


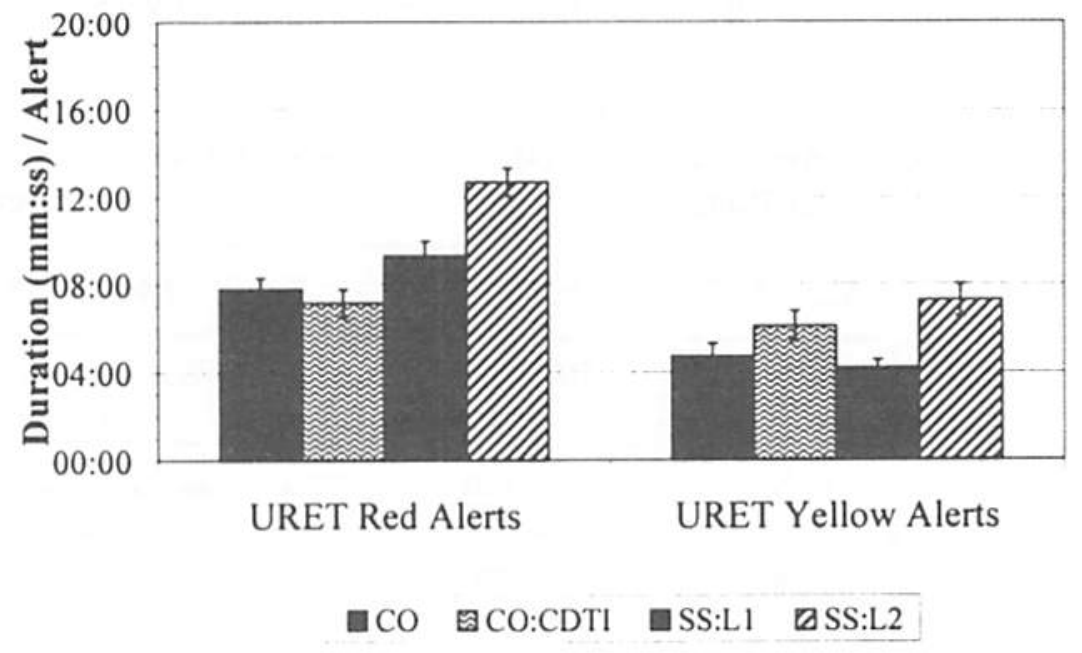

Figure 14. Mean duration of URET red and yellow conflict alerts.

\subsubsection{Loss of Separation for Conflicts Involving WJHTC Simulation Pilots.}

During the simulation, there were two separate instances where aircraft lost standard separation. The two separation violations occurred during SS:L2 runs. From an examination of the Host system data and videotapes, both instances were determined to be due to unintentional deviations from the simulation pilot scripts and not the result of shared-separation operations. The video tapes also confirmed that in both instances the controllers were aware that the aircraft were about to lose separation, but there was nothing they could do because they were not allowed to cancel free flight in the SS:L2 condition. One of the instances involved a planned conflict where a WJHTC simulation pilot entered a command into the system later than the scripted time resulting in a minimum horizontal separation distance of $4.87 \mathrm{~nm}$ at the same altitude. The second instance involved an unplanned conflict where a WJHTC simulation pilot descended an aircraft that was not scripted to change altitude resulting in a minimum horizontal separation distance of $4 \mathrm{~nm}$ and $900 \mathrm{ft}$ altitude separation.

\subsubsection{MSD Data and Free Flight Cancellations for Planned Conflicts Involving WJHTC}

\section{Simulation Pilots}

Table 13 and Table 14 show the number of conflicts and mean MSDs with SDs for altituderesolved and vector-resolved conflicts, respectively. MSD can be calculated by several methods. One popular method employs calculating slant range distances. Another method, the one used for this study, considers the perspective of the controller and computes the measurement in terms of the horizontal and vertical separation standards criteria. Therefore, MSD for an altituderesolved conflict represents the minimum horizontal distance between the aircraft pair until standard vertical separation was achieved (i.e., 1,000 ft below FL290 and 2,000 ft above FL290). The MSD for a vector-resolved conflict represents the minimum horizontal distance between aircraft at the same altitude. The MSDs for altitude-resolved conflicts were much greater for $\mathrm{CO}$ 
Table 13. Descriptive Statistics for Altitude-Resolved Planned Conflicts Involving WJHTC Simulation Pilots

\begin{tabular}{|c|c|c|c|c|c|c|}
\hline \multirow{2}{*}{$\begin{array}{c}\text { Control } \\
\text { Condition }\end{array}$} & \multicolumn{3}{|c|}{$\begin{array}{l}\text { Controller Altitude-Resolved } \\
\text { Conflicts }\end{array}$} & \multicolumn{3}{|c|}{$\begin{array}{l}\text { Scripted Pilot Altitude-Resolved } \\
\text { Conflicts }\end{array}$} \\
\hline & $\mathbf{N}$ & Mean MSD & SD & $\mathbf{N}$ & Mean MSD & SD \\
\hline $\mathrm{CO}$ & 16 & $32.5 \mathrm{~nm}$ & $19.7 \mathrm{~nm}$ & \multicolumn{3}{|c|}{$\begin{array}{l}\text { Pilots could not initiate maneuvers in this } \\
\text { condition }\end{array}$} \\
\hline CO:CDTI & 17 & $45.8 \mathrm{~nm}$ & $19.3 \mathrm{~nm}$ & \multicolumn{3}{|c|}{ Pilots could not initiate maneuvers in this } \\
\hline SS:Ll & 3* & $14.8 \mathrm{~nm}$ & $0.3 \mathrm{~nm}$ & 15 & $13.4 \mathrm{~nm}$ & $4.6 \mathrm{~nm}$ \\
\hline SS:L2 & \multicolumn{3}{|c|}{$\begin{array}{c}\text { Controllers could not cancel free flight in this } \\
\text { condition }\end{array}$} & 3 & $7.4 \mathrm{~nm}$ & $2.7 \mathrm{~nm}$ \\
\hline \multicolumn{7}{|c|}{$\begin{array}{l}\text { Note: The MSD for an altitude-resolved conflict represents the minimum horizontal distance } \\
\text { between the aircraft pair until standard vertical separation was achieved (i.e., } 1,000 \mathrm{ft} \text { below } \\
\text { FL290 and 2,000 ft above FL290). }\end{array}$} \\
\hline \multicolumn{7}{|c|}{ *Conflicts with cancelled free flight. } \\
\hline
\end{tabular}

Table 14. Descriptive Statistics for Controller Vector-Resolved Planned Conflicts Involving WJHTC Simulation Pilots

\begin{tabular}{|c|c|c|c|c|c|c|}
\hline \multirow{2}{*}{$\begin{array}{l}\text { Control } \\
\text { Condition }\end{array}$} & \multicolumn{3}{|c|}{$\begin{array}{l}\text { Controller Vector-Resolved } \\
\text { Conflicts }\end{array}$} & \multicolumn{3}{|c|}{$\begin{array}{l}\text { Scripted Pilot Vector-Resolved } \\
\text { Conflicts }\end{array}$} \\
\hline & $\mathbf{N}$ & Mean MSD & $S D$ & $\mathbf{N}$ & Mean MSD & $S D$ \\
\hline $\mathrm{CO}$ & 22 & $17.3 \mathrm{~nm}$ & $19.00 \mathrm{~nm}$ & \multicolumn{3}{|c|}{$\begin{array}{l}\text { Pilots could not initiate maneuvers in this } \\
\text { condition }\end{array}$} \\
\hline CO:CDTI & 20 & $15.7 \mathrm{~nm}$ & $10.16 \mathrm{~nm}$ & \multicolumn{3}{|c|}{$\begin{array}{l}\text { Pilots could not initiate manewvers in this } \\
\text { condition }\end{array}$} \\
\hline SS:L1 & 9* & $12.4 \mathrm{~nm}$ & $5.30 \mathrm{~nm}$ & 12 & $13.1 \mathrm{~nm}$ & $7.3 \mathrm{~nm}$ \\
\hline SS:L2 & \multicolumn{3}{|c|}{$\begin{array}{c}\begin{array}{c}\text { Controllers could not cancel free flight in this } \\
\text { condition }\end{array} \\
\end{array}$} & 36 & $10.0 \mathrm{~nm}$ & $7.2 \mathrm{~nm}$ \\
\hline \multicolumn{7}{|c|}{$\begin{array}{l}\text { Note: The MSD for a vector-resolved conflict represents the minimum horizontal distance } \\
\text { between aircraft at the same altitude. }\end{array}$} \\
\hline
\end{tabular}


and CO:CDTI compared to SS:L1 and SS:L2. Considering the means and SDs of MSDs for vector-resolved conflicts, it was not possible to establish if a difference exists between conditions. In SS:L1, controllers cancelled free flight and resolved the conflicts for 12 conflict pairs $(31 \%)$ and allowed WJHTC simulation pilots to resolve the conflicts for 27 pairs (69\%). Controllers used altitude to resolve three of the conflict pairs and used vectors to resolve nine of the pairs when free flight was cancelled. The results of the ANOVAs for MSDs are reported in Appendix G, Section G-4.

\subsubsection{Information Requirements and Procedures}

\subsubsection{Controller Ratings for the Amount of Information Available to Resolve Conflicts}

Figure 15 shows mean controller ratings $( \pm 1$ SEM) for information available to resolve conflicts. In general, controllers rated the amount of information to resolve conflicts as adequate, and there were no differences between the four conditions. The results of the ANOVA are reported in Appendix G, Section G-5.

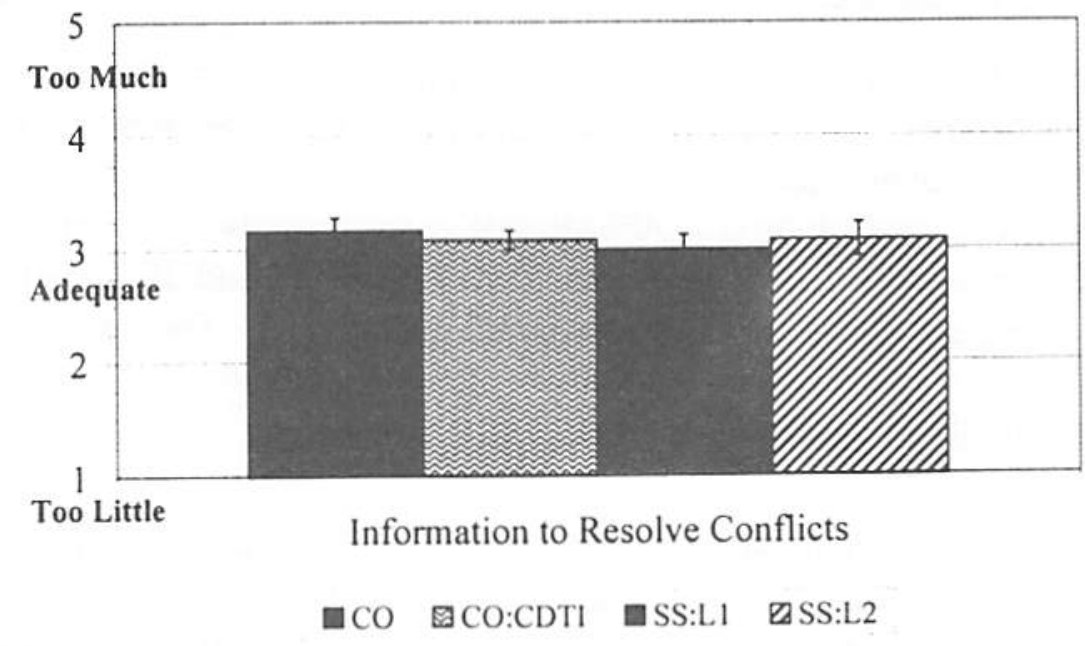

Figure 15. Controller mean ratings of information to resolve conflicts.

\subsubsection{Controller Ratings for URET Conflict Alert Timeliness}

Figure 16 shows mean controller ratings ( \pm 1 SEM) for URET conflict alert timeliness. In general, controllers rated the timing of the conflict alerts as adequate and there were no differences between the four conditions. The results of the ANOVA are reported in Appendix G, Section G-6. 


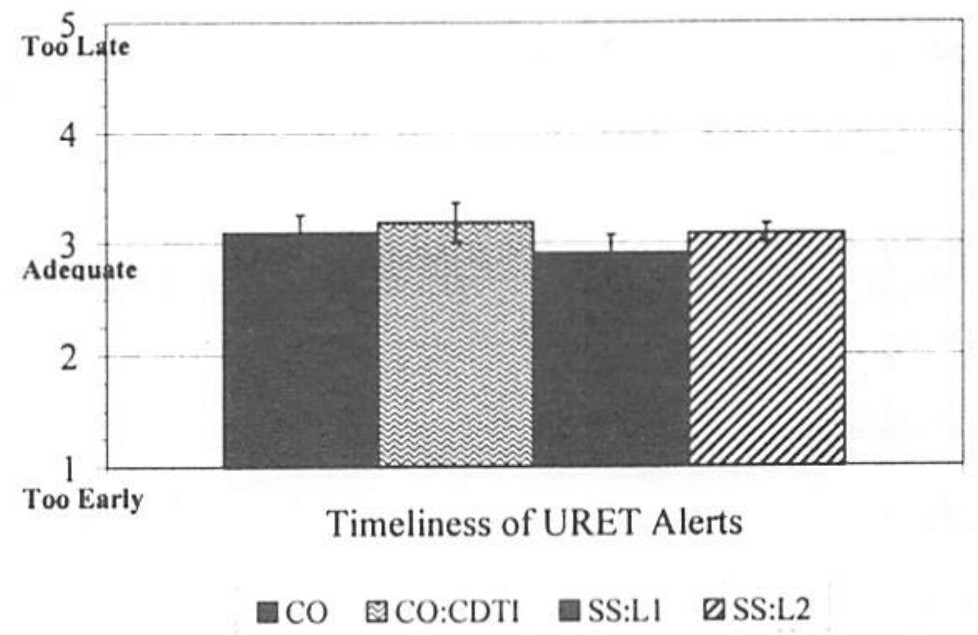

Figure 16. Controller mean ratings for URET conflict alert timeliness.

\subsubsection{Controller Ratings for How Often They Monitored Air $\leftrightarrow$ Air Communications}

Table 15 shows the frequencies and percentages of controller responses for ratings of how often they monitored air $\leftrightarrow$ air communications. The majority of controllers reported that they always monitored air $\leftrightarrow$ air communications, and there was no difference between SS:L1 and SS:L2. In both SS:L1 and SS:L2, three of the six (50\%) R-side controllers always monitored and three $(50 \%)$ never monitored air $\leftrightarrow$ air communications. In both SS:L1 and SS:L2, all six $(100 \%)$ of the D-side controllers always monitored air $\leftrightarrow$ air communications. The researchers did not perform an ANOVA on controller ratings for how often they monitored air $\leftrightarrow$ air communications due to lack of variability in the data.

Table 15. Controller Frequencies of Air $\leftrightarrow$ Air Communication Monitoring

\begin{tabular}{|l|c|c|c|c|c|c|c|c|c|c|}
\hline & \multicolumn{4}{|c|}{ R-Side Controllers } & \multicolumn{5}{c|}{ D-Side Controllers } \\
\cline { 2 - 12 } $\begin{array}{l}\text { Control } \\
\text { Condition }\end{array}$ & $\begin{array}{c}1 \\
\text { Never }\end{array}$ & 2 & $\begin{array}{c}3 \\
\text { Some }\end{array}$ & 4 & $\begin{array}{c}5 \\
\text { Always }\end{array}$ & $\begin{array}{c}1 \\
\text { Never }\end{array}$ & 2 & $\begin{array}{c}3 \\
\text { Some }\end{array}$ & $\mathbf{4}$ & $\begin{array}{c}5 \\
\text { Always }\end{array}$ \\
\hline SS:L1 & $3(50 \%)$ & 0 & 0 & 0 & $3(50 \%)$ & 0 & 0 & 0 & 0 & $6(100 \%)$ \\
\hline SS:L2 & $3(50 \%)$ & 0 & 0 & 0 & $3(50 \%)$ & 0 & 0 & 0 & 0 & $6(100 \%)$ \\
\hline
\end{tabular}




\subsubsection{Controller Ratings for the Usefulness of Monitoring Air $\leftrightarrow$ Air Communications}

Figure 17 shows mean controller ratings ( \pm 1 SEM) for the usefulness of monitoring air $\leftrightarrow$ air communications. Only the ratings from controllers who monitored air $\leftrightarrow$ air communications are represented in this figure. In general, controllers rated monitoring air $\leftrightarrow$ air communications as useful, and there was no significant difference between SS:L1 and SS:L2. In addition, there was no significant difference between R-side and D-side controllers. The results of the ANOVA are reported in Appendix G, Section G-7.

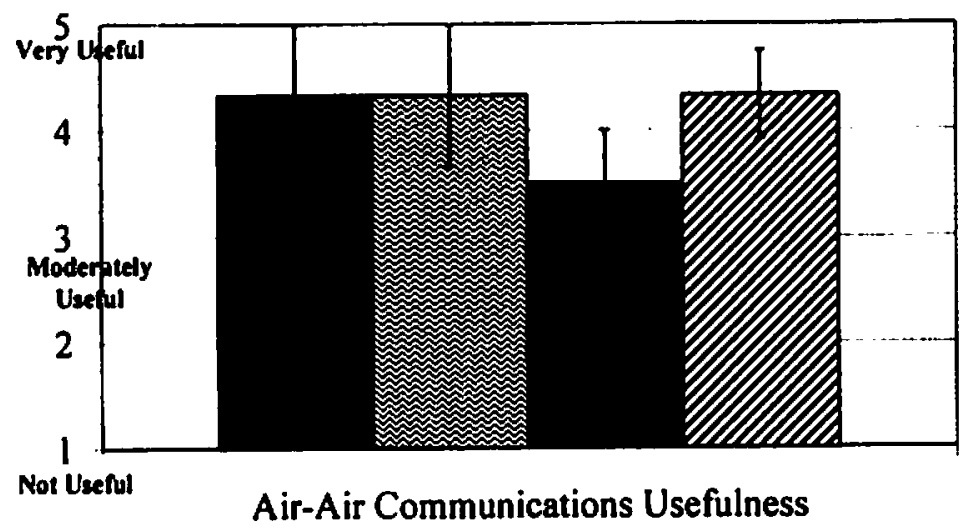

QSS:L1, R-Side QSS:L2, R-Side DSS:LI, D-Side OSS:L2, D-Side

Figure 17. Controller mean ratings for usefulness of air $\leftrightarrow$ air communications.

\subsubsection{Controller Ratings for the Helpfulness of the Shared-Separation Concept}

Figure 18 shows mean controller ratings $( \pm 1 \mathrm{SEM})$ for the helpfulness of the shared-separation concept. In general, controllers rated the shared-separation concept as not helpful, and there was no significant difference between SS:L1 and SS:L2. The results of the ANOVA are reported in Appendix G, Section G-8. 


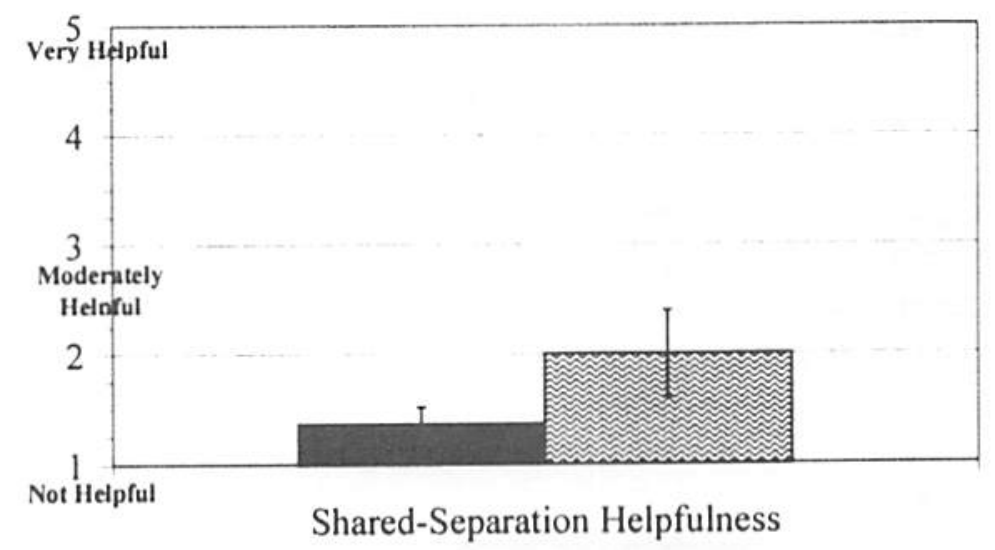

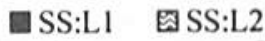

Figure 18. Controller mean ratings for the helpfulness of shared-separation.

\subsubsection{URET Trial Plans}

Figure 19 shows mean frequencies $( \pm 1$ SEM) of URET trial plans. In general, controllers formed more trial plans in CO and CO:CDTI compared to SS:L1 and SS:L2. The results of the ANOVA are reported in Appendix G, Section G-9.

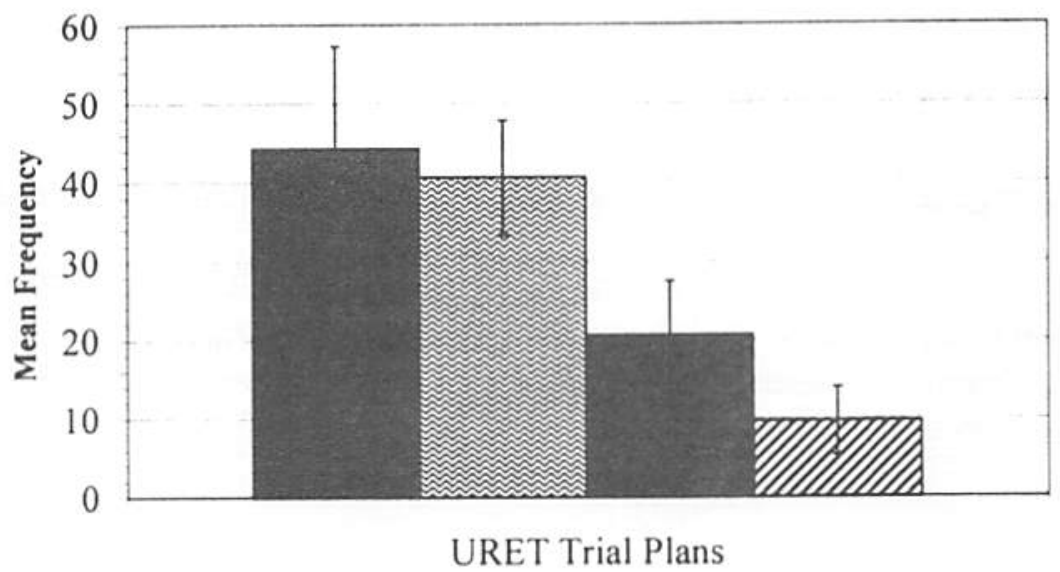

$\square \mathrm{CO}$ 圂CO:CDTI $\square \mathrm{SS}: \mathrm{Ll} \square \mathrm{SS}: \mathrm{L} 2$

Figure 19. Mean frequency of URET trial plans. 


\subsubsection{Controller Workload and Situation Awareness}

\subsubsection{Controller Ratings for Physical, Mental, and Overall Workload}

Figure 20 shows mean controller ratings ( \pm 1 SEM) for physical, mental, and overall workload. In general, controllers rated their workload as moderate. Mental and overall workload ratings were higher for SS:L1 compared to the other conditions. The results of the ANOVAs are reported in Appendix G, Section G-10.

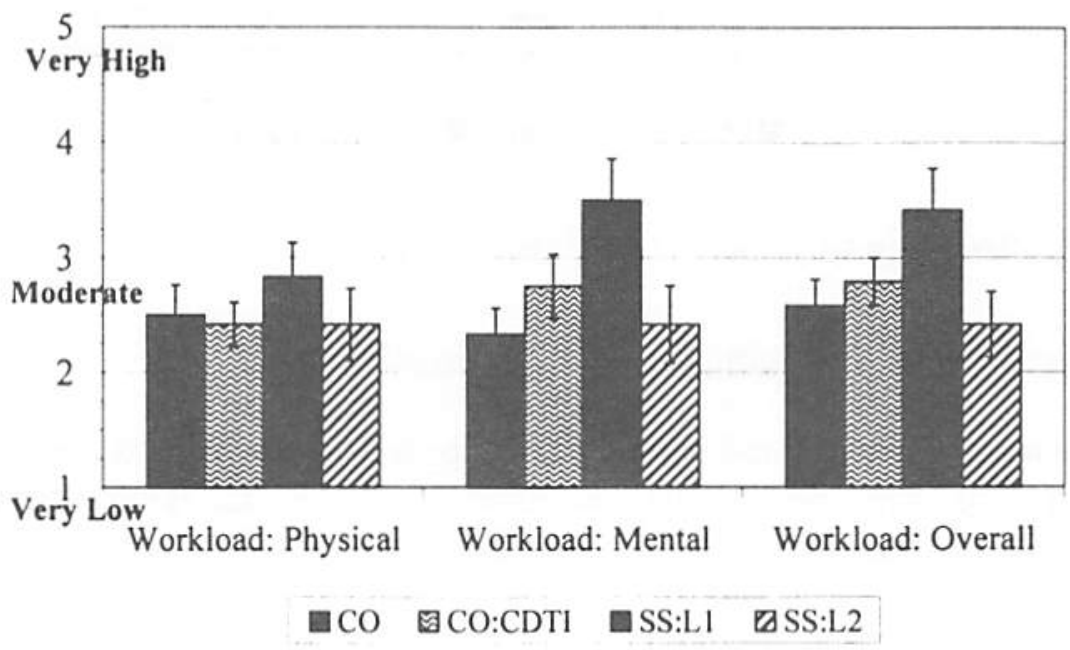

Figure 20. Controller mean workload ratings: physical, mental, and overall.

\subsubsection{Controller Workload Ratings for Maintaining Aircraft Separation, Land Line} Coordination, R-Side-to-D-Side Coordination, Ground $\rightarrow$ Air Transmissions, and URET Coordination

Figure 21 shows mean controller workload ratings ( \pm 1 SEM) for maintaining aircraft separation, land line coordination, R-side-to-D-side position coordination, ground $\rightarrow$ air transmissions, and URET coordination. In general, controllers rated their workload for these specific areas as ranging from low to moderate. Controller workload was generally the highest for maintaining aircraft separation and the lowest for URET coordination. There were no significant differences in workload among the four conditions for these specific measures. The results of the ANOVAs are reported in Appendix G, Section G-11. 


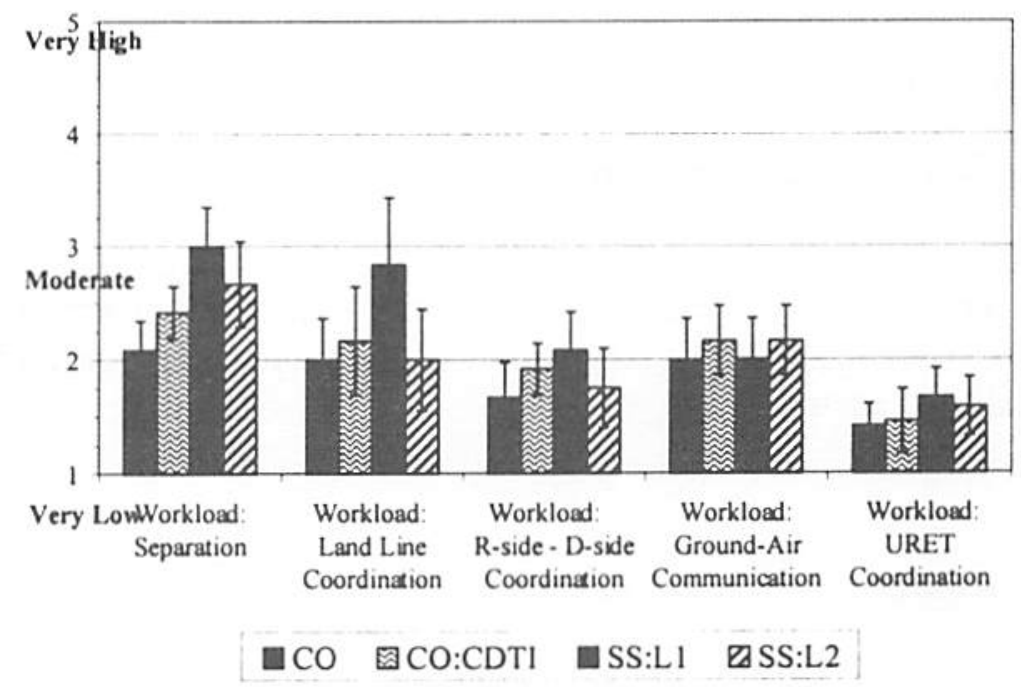

Figure 21. Controller mean workload ratings: specific measures.

\subsubsection{Controller Ratings for Feeling Rushed and Bored}

Figure 22 shows mean controller ratings ( \pm 1 SEM) for feeling rushed and bored during the simulation. In general, controllers felt neither rushed nor bored, and there were no significant differences between the four conditions. The results of the ANOVAs are reported in Appendix G, Section G-12.

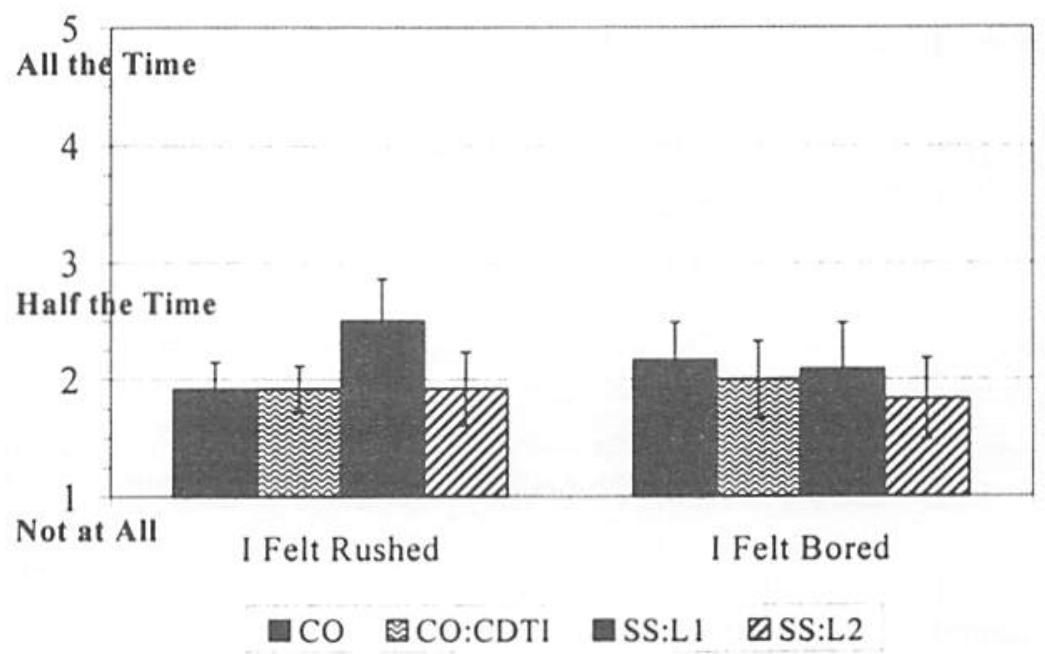

Figure 22. Controller mean ratings for feeling rushed and bored. 


\subsubsection{Controller Ratings of Overall Situation Awareness}

Figure 23 shows mean controller ratings ( \pm 1 SEM) of overall situation awareness. In general, controllers rated their overall situation awareness as high and there were no significant differences between the four conditions. The results of the ANOVA are reported in Appendix G, Section G-13.

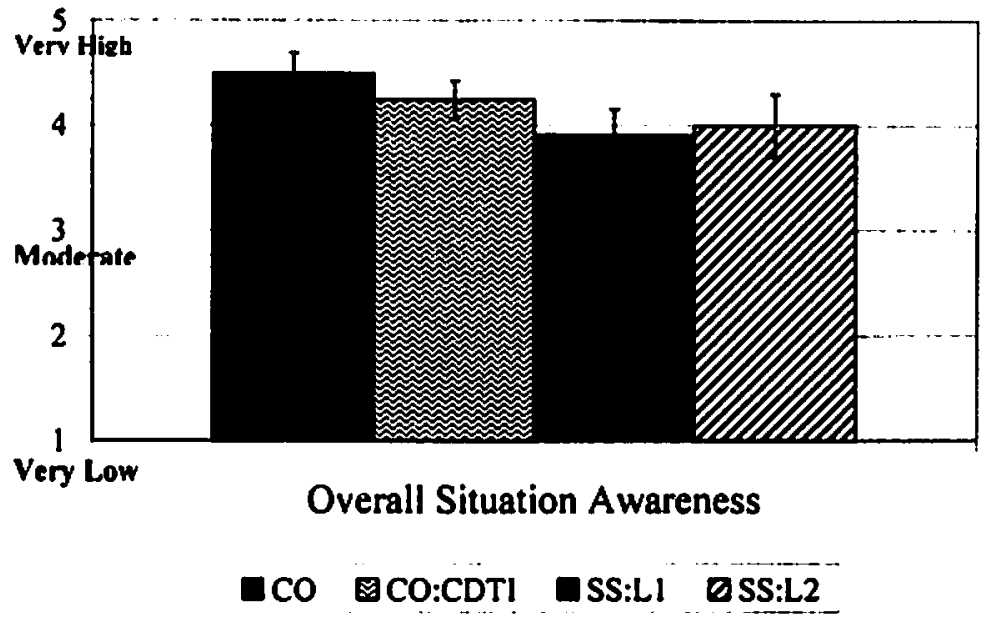

Figure 23. Controller mean ratings of overall situation awareness.

\subsubsection{Controller Interval Workload Ratings}

Figure 24 and Figure 25 show mean interval workload ratings for the R-side and D-side controllers, respectively. In general, controllers rated their workload using the WAK tool as rather low. As shown in the figures, lower and higher workload periods can be seen for each of the four conditions. Each condition had two peak workload periods: the first occurred at approximately 25-35 minutes into the run, and the second was at about 60-70 minutes into the run. In contrast to the results for the controller ratings of overall workload (section 5.1.3.1), there were only small differences between the four conditions for interval workload ratings. The results of the ANOVA for mean workload ratings collapsed across the intervals are reported in Appendix G, Section G-14. 

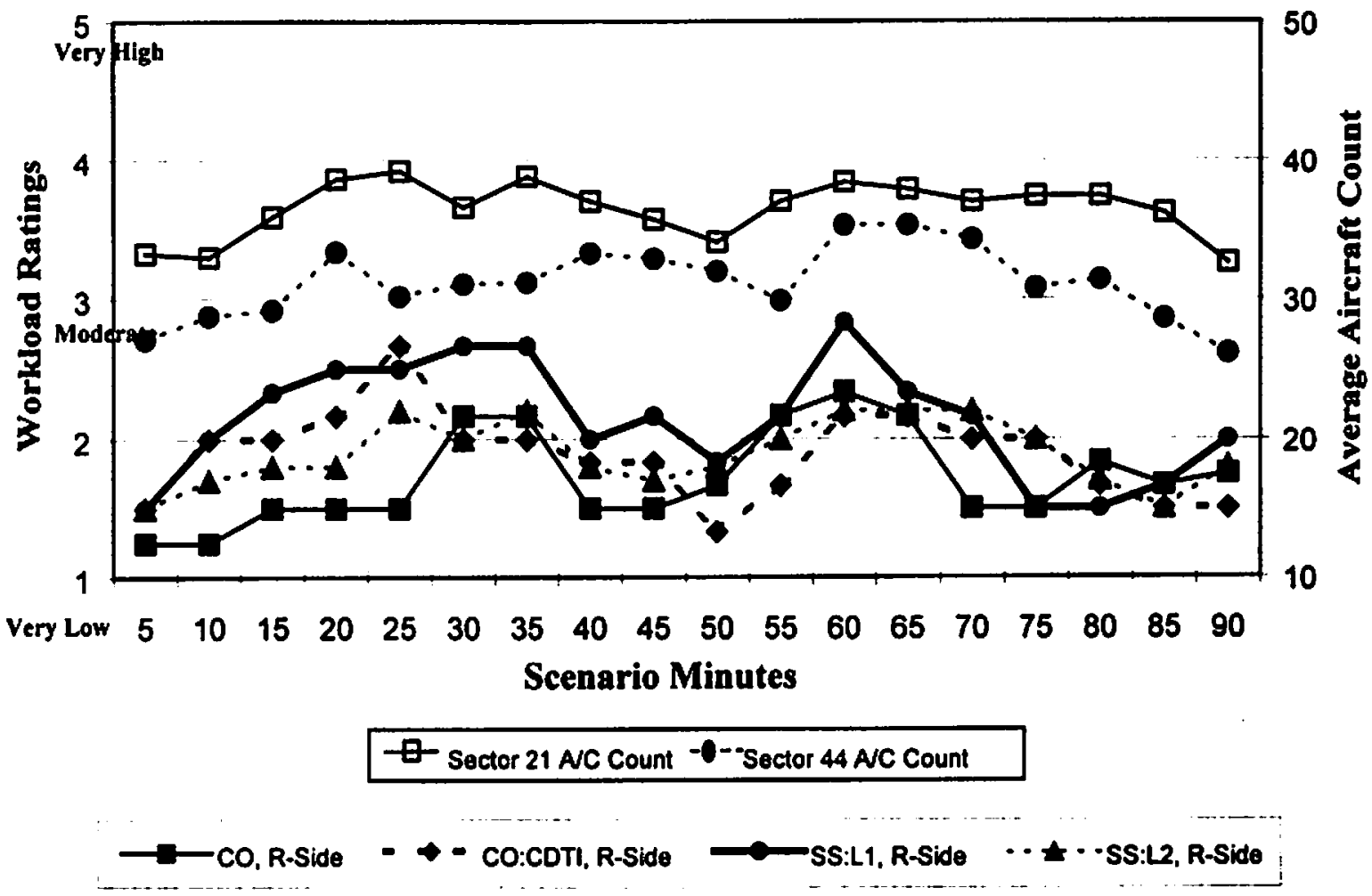

Figure 24. R-side controller mean ratings for interval workload. 


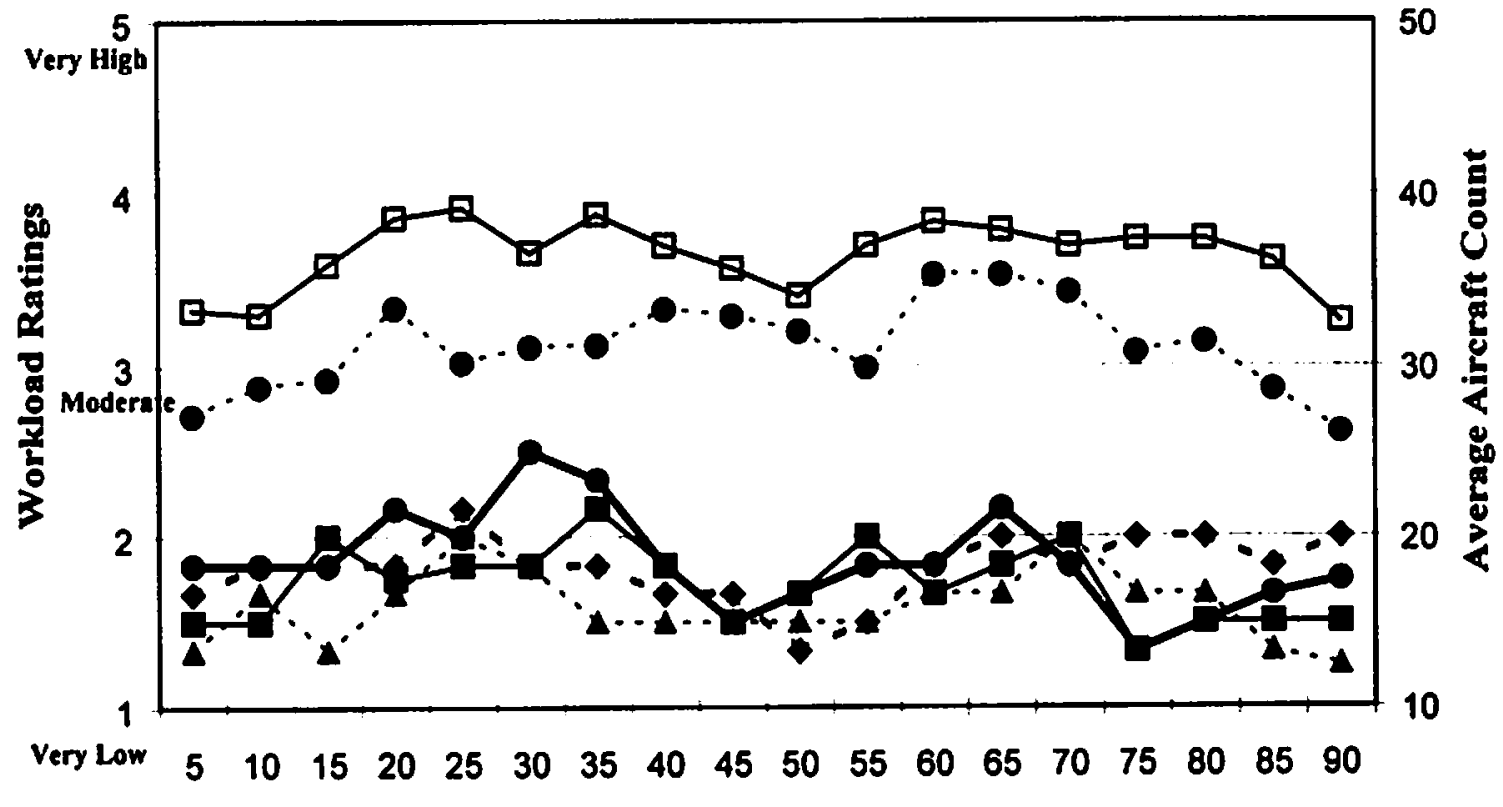

Scenario Minutes

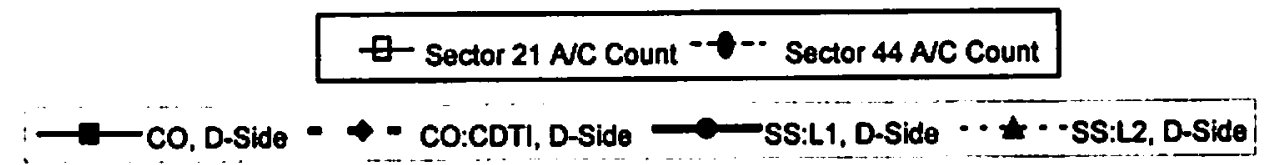

Figure 25. D-side controller mean ratings for interval workload.

\subsubsection{Expert Observer Ratings of Controller Physical Taskload}

Figure 26 shows mean EO ratings ( \pm 1 SEM) of controller physical taskload. In general, EOs rated controller physical taskload as moderate. Similar to the results of controller ratings for overall workload, EO ratings for controller physical taskload were higher in SS:Ll compared to the other conditions. The results of the ANOVA are reported in Appendix G, Section G-15. 


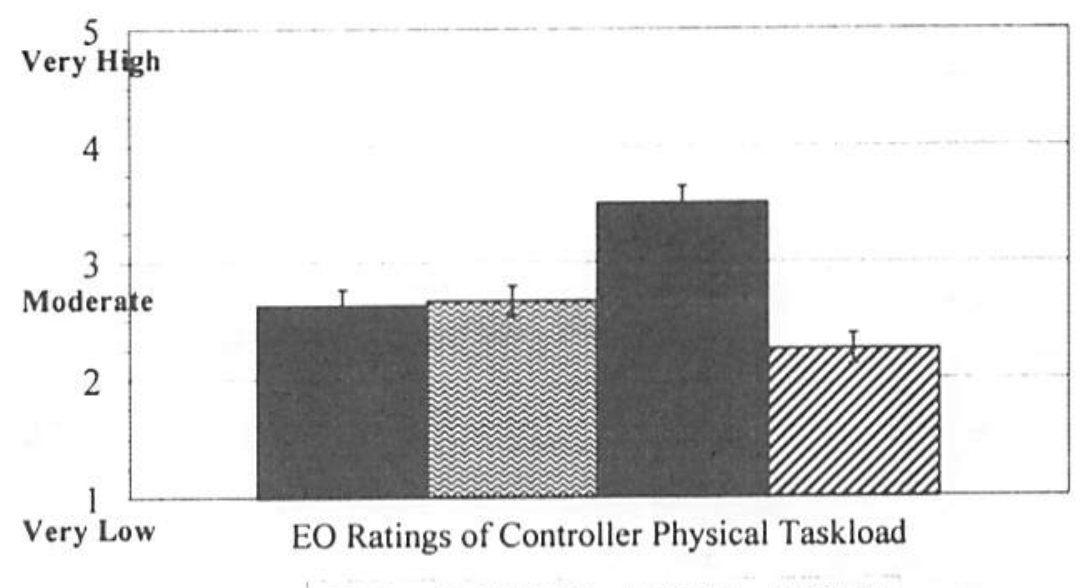

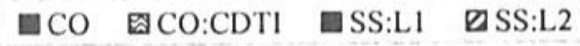

Figure 26. Expert Observer mean ratings of controller physical taskload.

\subsubsection{Controller Ground $\rightarrow$ Air and Land Line Push-to-Talk Transmissions}

Figure 27 shows mean frequencies $( \pm 1$ SEM) of ground $\rightarrow$ air and land line PTTs. There were many more ground $\rightarrow$ air PTTs than land line PTTs, and both types were the lowest in SS:L2. It should be noted that by design, SS:L2 had no scripted pilot inquires, requests, or intent information relays (and, therefore no controller responses or acknowledgments). In addition, controllers could not cancel free or issue control instructions to pilots. These factors likely contributed to the decrease in SS:L2 PTTs. The results of the ANOVAs are reported in Appendix G, Section G-16A.

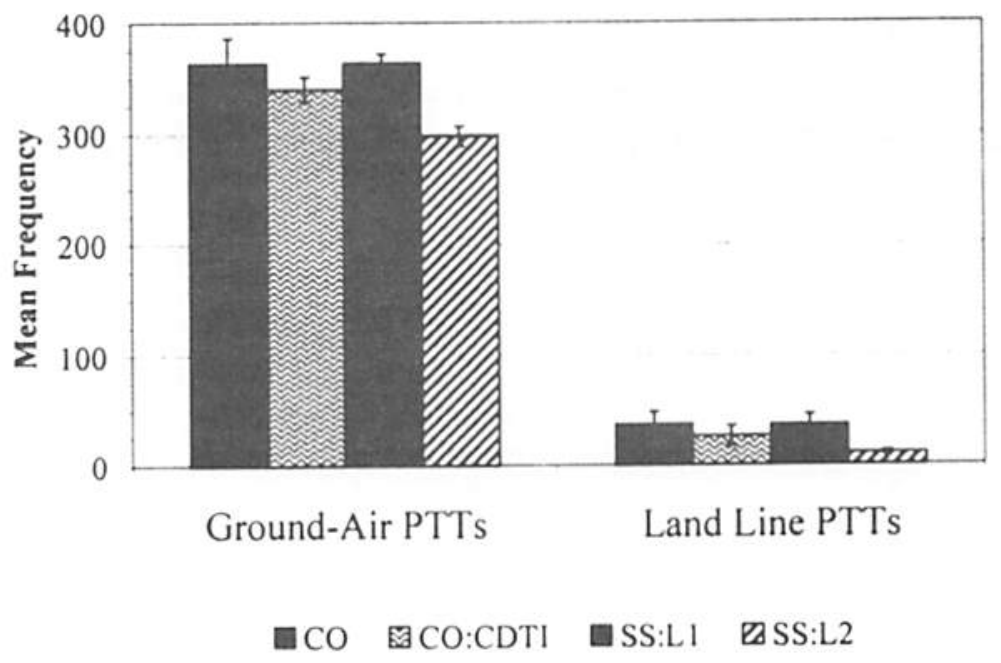

Figure 27. Mean frequency of ground $\rightarrow$ air and land line PTTs. 
Figure 28 shows mean duration per transmission $( \pm 1$ SEM) of ground $\rightarrow$ air and land line PTTs. Ground $\rightarrow$ air PTTs were shorter than land line PTTs, and there were only small differences between the four conditions. The results of the ANOVAs are reported in Appendix G, Section G-16B.

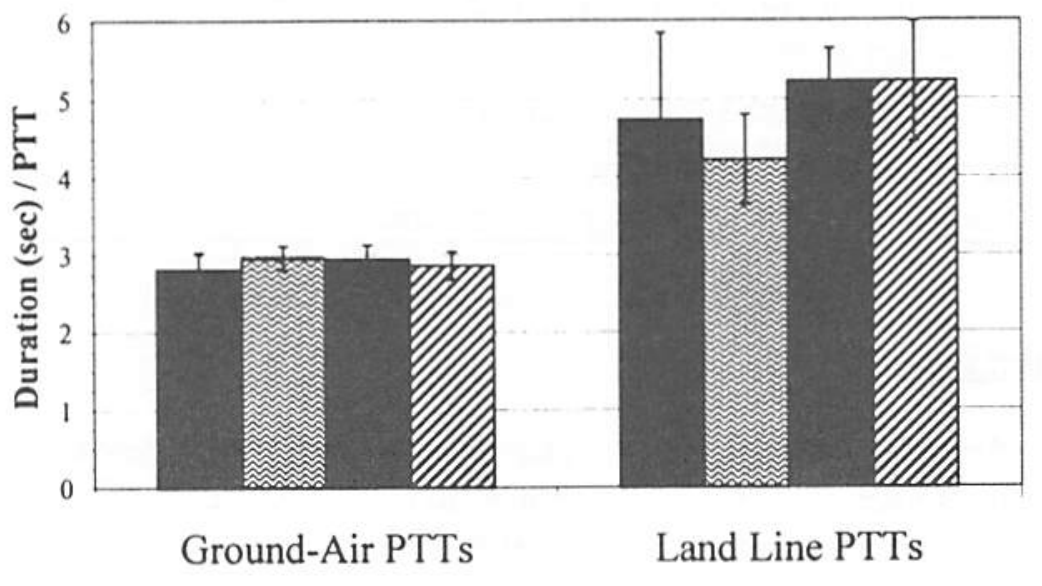

$\square \mathrm{CO} \approx \mathrm{CO} \mathrm{CDTl} \square \mathrm{SS}: \mathrm{Ll} \square \mathrm{SS}: \mathrm{L} 2$

Figure 28. Mean duration per transmission of ground $\rightarrow$ air and land line PTTs.

\subsubsection{Exit Form Responses and Ratings}

Tables 16 and 17 show controller responses about separation responsibility confusion and ratings of simulation realism and training obtained from the Exit Form. Nine controllers reported that they were not confused about who had the separation responsibility. However, three controllers reported that they were confused at some time but did not indicate how long. In general, controllers rated the realism of the simulation pilot responses and overall realism of the simulation as moderate. In addition, controllers rated the simulation training as moderate to adequate.

Table 16. Controller Role and Separation Responsibility Confusion

\begin{tabular}{|l|c|c|}
\hline \multicolumn{1}{|c|}{$\mathrm{N}=12$} & YES & NO \\
\hline $\begin{array}{l}\text { Confused at any time about } \\
\text { separation responsibility and role }\end{array}$ & $\mathrm{n}=3$ & $\mathrm{n}=9$ \\
$(\mathrm{R}-\mathrm{side}=2 \& \mathrm{D}$-side $=1)$ & $(25 \%)$ & $(\mathrm{R}$-side $=4$ \& D-side $=5)$ \\
\hline
\end{tabular}


Table 17. Controller Mean Ratings for Simulation Realism and Training

\begin{tabular}{|c|c|c|}
\hline $\mathrm{N}=12$ & $\mathrm{M}$ & $\mathrm{SD}$ \\
\hline $\begin{array}{c}\text { Realism of the simulated flight crew responses } \\
(l=\text { very unrealistic, 3 = moderate, } 5=\text { very realistic })\end{array}$ & 3.3 & 0.9 \\
\hline $\begin{array}{c}\text { Overall realism of the simulation } \\
(1=\text { very unrealistic, 3 = moderate, } 5=\text { very realistic })\end{array}$ & 3.0 & 0.9 \\
\hline $\begin{array}{c}\text { Adequacy of simulation training } \\
(l=\text { inadequate, 3 = moderate, } 5=\text { adequate }\end{array}$ & 3.9 & 1.1 \\
\hline
\end{tabular}

\subsection{Air-Side Results}

Due to the limited number of participants in this study $\mathrm{N}=3$ flight crews for a total of 6 pilot participants), no inferential statistics were applied to the air-side data. Simulation pilots performing scripted events operated the other aircraft in this investigation; therefore, those data will not be discussed in this section.

Descriptive statistics are provided for the various measures collected during the study. In addition, Pilot Exit Form data were analyzed using the Analytical Hierarchy Process (AHP). AHP compared ratings of measures across conditions on a mirrored 9-point scale. AHP factor loadings were compared using a standard "level of dominance" scale where $(l=$ equal, $3=$ weak, $5=$ strong, $7=$ very strong, and $\geq 9=$ an absolute preference). These ratings indicated the dominance of one condition over another in terms of pilot preference. Therefore, a factor of 1 would indicate that both of the conditions being compared were equally preferred (or no preference), and a factor of 9 would indicate an absolute (or overwhelming) preference for one of the conditions over the other.

Except where noted, statistics summarize across the three, 20-minute flight segments in each of the four conditions. Thus, there were 36 flight segments considered for most of these data summaries. These data must be interpreted with caution because of the small number of participants.

\subsubsection{Operational Issues that Affect Shared-Separation Operations}

\subsubsection{Safety Measures}

- Loss of Separation for Conflicts Involving the NASA ARC Simulator.

In the two shared-separation conditions (SS:L1 and SS:L2), the flight crews had varying levels of separation responsibility. For all conditions, the minimum separation distance was defined as either $5 \mathrm{~nm}$ horizontal or $1000 / 2000 \mathrm{ft}$ vertical (as appropriate). There were no NASA flight segments in which minimum separation standards were violated by the flight crews in the four conditions (CO, CO:CDTI, SS:L1, SS:L2). In addition, there were no flight segments in which the flight crews received any TCAS alerts. 
- AHP Flight Safety Ratings.

Pilot participants were asked which condition they preferred in terms of flight safety. Table 18 shows the AHP results of these data. Interestingly, AHP preference ratings indicated that pilots preferred the conditions in which they assumed some responsibility for separation over the more traditional roles (SS:L2 to CO by a factor of 9.5 and SS:L2 over SS:L1 by a factor of 2.9). The least favored conditions were $\mathrm{CO}$ and $\mathrm{CO}: \mathrm{CDTI}$.

Table 18. Pilot Participant AHP Preference Ratings for Flight Safety

\begin{tabular}{|l|c|c|}
\hline Flight Safety Comparison & Factor & Level of dominance \\
\hline CO:CDTI equal to CO & 1.4 & equal \\
\hline SS:L1 preferred over CO & 3.3 & weak \\
\hline SS:L2 preferred over CO & 9.5 & absolute \\
\hline SS:L1 preferred over CO:CDTI & 2.3 & weak \\
\hline SS:L2 preferred over CO:CDTI & 6.7 & very strong \\
\hline SS:L2 preferred over SS:L1 & 2.9 & weak \\
\hline
\end{tabular}

\subsubsection{Flight Crew Performance}

- Flight Crew Conflict Detection.

Flight crews could identify potential conflicting aircraft using the information provided by the CDTI-AL. For each of the 27 flight segments in which this information was available (those from CO:CDTI, SS:L1, and SS:L2), video and audio tapes from the flight deck were coded by two researchers to determine the time at which the intruder aircraft was identified as a possible conflict by the flight crews. Although the flight crews were not given separation flexibility in CO:CDTI, they did appear to monitor their CDTI-AL in that condition. Conflict detection time was defined as duration from the time the intruder aircraft appeared on the CDTI-AL (at the beginning of each flight segment) until both members of the flight crew indicated that the aircraft was a potential problem (also determined by video transcription analysis). In 26 out of the 27 flight segments with CDTI-AL information, the intruder aircraft was identified by the flight crew as a potential conflict. In the remaining sun, the flight crew made a horizontal maneuver to go direct to a subsequent fix along their route. It was unclear whether the maneuver was made because of the potential for conflict with the intruder aircraft, for fuel efficiency, or for both. This maneuver resolved the conflict, so no conflict detection time was generated. Table 19 shows mean conflict detection times and SDs. These average times were at least 4 minutes before the time that the intruder aircraft triggered an alert on the CDTI-AL. The pilot participants detected all conflicts prior to a CDTI-AL alert indication. It should be noted that the conflicts all had the 
same general angle and location, and the pilot participants did indicate that they identified this pattern during the experiment.

Table 19. Pilot Participant Mean Intruder-Detection Times

\begin{tabular}{|l|c|c|c|c|c|c|}
\hline \multirow{2}{*}{\multicolumn{1}{|c|}{ N =6 }} & \multicolumn{2}{|c|}{ CO:CDTI } & \multicolumn{2}{c|}{ SS:L1 } & \multicolumn{2}{c|}{ SS:L2 } \\
\cline { 2 - 7 } & $M$ & SD & $M$ & SD & $M$ & SD \\
\hline $\begin{array}{l}\text { Pilot Intruder Aircraft } \\
\text { Detection Times (min:sec) }\end{array}$ & $2: 17$ & $1: 30$ & $1: 48$ & $1: 41$ & $1: 34$ & $1: 28$ \\
\hline
\end{tabular}

In addition to determining how long flight crews took to identify the conflicting aircraft, following each condition they were asked to rate 1) the ease of detecting a conflict prior to an alert indication on the CDTI-AL or controller advisory, and 2) the effectiveness of the CDTI-AL for use in a shared-separation environment. The means and SDs are presented in Tables 20 and 21. Similar to the flight crew conflict detection times, inspection of the subjective ratings data suggest that flight crews found it easy to identify the intruder aircraft in all three of the conditions that included the CDTI-AL, and that the CDTI-AL was an effective tool in identifying the conflicting aircraft.

Table 20. Pilot Participant Mean Ratings of the Ease of Detecting Conflicts Prior to Alert or Controller Advisory

\begin{tabular}{|c|c|c|c|c|c|c|}
\hline \multirow{2}{*}{$\mathrm{N}=6$} & \multicolumn{2}{|c|}{ CO:CDTI } & \multicolumn{2}{c|}{ SS:L1 } & \multicolumn{2}{c|}{ SS:L2 } \\
\cline { 2 - 8 } & $\mathrm{M}$ & SD & $\mathrm{M}$ & SD & $\mathrm{M}$ & SD \\
\hline $\begin{array}{l}\text { Ease of detecting conflicts prior } \\
\text { to alert or controller advisory }\end{array}$ & 3.7 & 1.5 & 3.7 & 1.5 & 4.7 & 0.5 \\
\hline
\end{tabular}

$(I=$ not easy, $3=$ moderately easy, $5=$ very easy) 
Table 21. Pilot Participant Mean Ratings of the CDTI-AL Effectiveness for Shared-Separation

\begin{tabular}{|l|c|c|c|c|}
\hline \multirow{2}{*}{$\mathrm{N}=6$} & \multicolumn{2}{|c|}{ SS:L1 } & \multicolumn{2}{c|}{ SS:L2 } \\
\cline { 2 - 5 } & $\mathrm{M}$ & SD & $\mathrm{M}$ & SD \\
\hline $\begin{array}{l}\text { Effectiveness of display for } \\
\text { shared-separation }\end{array}$ & 4.2 & 1.0 & 4.3 & 0.8 \\
\hline
\end{tabular}

( $I=$ not effective, $3=$ moderately effective, $5=$ very effective $)$

\subsubsection{Flight Efficiency Measures}

- Fuel burn.

Because of the different routes through the sectors and due to the short flight segments, fuel burn comparisons could not be made among the conditions. Future studies need to consider longer flight segments in order to estimate potential impacts of fuel burn in various sharedseparation conditions.

- Pilot participant ratings of flight efficiency.

After flying all of the conditions, flight crews were asked to rate the flight efficiency of each procedural condition using the AHP. Pilot participants were asked which authority conditions they considered better for flight efficiency. Again, as can be seen in Table 22, the pilots overwhelmingly preferred SS:L2 over $C O$ and CO:CDTI. They preferred SS:L2 over CO by a factor of 12.2, over CO:CDTI by a factor of 10.1 and over SS:L1 by a factor of 3.2. SS:LI was preferred over CO and CO:CDTI by factors of 3.8 and 3.1, respectively.

Table 22. Pilot Participant Preference for Flight-Efficiency

\begin{tabular}{|l|c|c|}
\hline \multicolumn{1}{|c|}{ Flight Efficiency Comparison } & Factor & Level of dominance \\
\hline CO:CDTI equal to CO & 1.2 & equal \\
\hline SS:L1 preferred over CO & 3.8 & weak \\
\hline SS:L2 preferred over CO & 12.2 & absolute \\
\hline SS:L1 preferred over CO:CDTI & 3.1 & weak \\
\hline SS:L2 preferred over CO:CDTI & 10.1 & absolute \\
\hline SS:L2 preferred over SS:L1 & 3.2 & weak \\
\hline
\end{tabular}




\subsubsection{Flight Crew Communication}

- Air $\leftrightarrow$ Ground Communication.

After flying in each condition, pilot participants were asked to rate how much time they felt was available for air $\leftrightarrow$ ground communications. Table 23 depicts their mean ratings and $S D s$ for each condition. Across all conditions, pilots reported an adequate amount of time available for air $\leftrightarrow$ ground communications.

Table 23. Pilot Participant Mean Ratings for Time Available for Air $\leftrightarrow$ Ground Communication

\begin{tabular}{|c|c|c|c|c|c|c|c|c|}
\hline \multirow[b]{2}{*}{$N=6$} & \multicolumn{2}{|c|}{$\mathrm{CO}$} & \multicolumn{2}{|c|}{ CO:CDTI } & \multicolumn{2}{|c|}{ SS:LI } & \multicolumn{2}{|c|}{ SS:L2 } \\
\hline & $\mathbf{M}$ & $S D$ & $\mathbf{M}$ & $S D$ & $\mathbf{M}$ & $S D$ & $\mathbf{M}$ & $S D$ \\
\hline $\begin{array}{l}\text { Amount of time } \\
\text { available for } \\
\text { air } \leftrightarrow \text { ground } \\
\text { communication }\end{array}$ & 3.3 & 1.0 & 3.2 & 0.4 & 3.0 & .0 & 3.2 & 0.4 \\
\hline
\end{tabular}

$(I=$ too little, $3=$ adequate, $5=100$ much $)$

- Air $\leftrightarrow$ Air Communication.

In SS:L1 and SS:L2, an air $\leftrightarrow$ air frequency was available on which all pilots (pilot participants and simulation pilots) were able to communicate among themselves. Because the simulation pilots were confederates of the study, their communication duration times were not calculated. Simulation pilots did not initiate communication with the pilot participants (scripted or otherwise). Pilot participants contacted the intruder aircraft in only three (33\%) of the nine flight segments in SS:Ll and in four (44\%) of the nine flight segments in SS:L2. Pilot participants contacted one other aircraft in SS:L1 and none of the surrounding aircraft other than the intruder in SS:L2.

Following each condition, pilot participants were asked to rate several questions concerning air $\leftrightarrow$ air communication; how often they monitored the air $\leftrightarrow$ air frequency, the usefulness of air $\leftrightarrow$ air communication, and the amount of time available for monitoring air $\leftrightarrow$ air communication. Table 24 summarizes pilot participant responses for all questions. In SS:Ll, pilots reported that they frequently monitored the communications between the other pilots and they sometimes monitored it in SS:L2. Pilots reported that, on average, monitoring the air $\leftrightarrow$ air frequency was moderately useful, and they had an adequate amount of time to monitor the air $\leftrightarrow$ air frequency in both shared-separation conditions. 
Table 24. Pilot Participant Mean Ratings Related to Air $\leftrightarrow$ Air Communication

\begin{tabular}{|c|c|c|c|c|}
\hline \multirow{2}{*}{ N=6 } & \multicolumn{2}{|c|}{ SS:L1 } & \multicolumn{2}{c|}{ SS:L2 } \\
\cline { 2 - 5 } & $\mathrm{M}$ & SD & M & SD \\
\hline $\begin{array}{c}\text { How often monitored other air } \leftrightarrow \text { air communication } \\
(l=\text { never, 3 = sometimes, 5 = always })\end{array}$ & 4.0 & 0.9 & 3.3 & 0.8 \\
\hline $\begin{array}{c}\text { Usefulness of monitoring other air } \leftrightarrow \text { air communication } \\
(1=\text { not useful, 3 = moderately useful, 5 = very useful })\end{array}$ & 3.0 & 1.1 & 2.7 & 0.5 \\
\hline $\begin{array}{c}\text { Amount of time available to monitor air } \leftrightarrow \text { air frequency } \\
(I=\text { too little, 3 = adequate, 5 = too much })\end{array}$ & 2.8 & 0.4 & 3.3 & 0.5 \\
\hline
\end{tabular}

\subsubsection{Information Requirements and Procedures}

\subsubsection{Procedures}

During the study, flight crews flew three conditions in which different procedures and/or new technologies were provided as well as a fourth condition using current operational procedures. Flight crews were briefed on both the procedures for themselves as pilots and on the new procedures for the controllers. After flying in each condition, flight crews were queried about the procedures used for shared-separation in this study; means and SDs for their form responses are presented in Table 25. According to these responses, flight crews felt that the operations used in both SS:L1 and SS:L2 were helpful for performing their jobs, and they felt comfortable sharing the separation responsibility with controllers. Pilot participants also reported only slight confusion about who had separation authority during the runs. Table 26 shows the pilots mean ratings and SDs. This confusion was also noticed in a few comments by the pilots (found during videotape analyses). Finally, pilot participants indicated that the pilot right-of-way rules were useful for negotiation and initiating contact with conflicting aircraft $(\mathrm{M}=4.0, S D=1.1 ; l=$ not useful, 3 = moderately useful, $5=$ very useful). 
Table 25. Pilot Participant Mean Ratings of Shared-Separation

\begin{tabular}{|c|c|c|c|c|}
\hline \multirow{2}{*}{ N=6 } & \multicolumn{2}{|c|}{ SS:L1 } & \multicolumn{2}{c|}{ SS:L2 } \\
\cline { 2 - 6 } & M & SD & M & SD \\
\hline $\begin{array}{l}\text { Impact of shared-separation operations on performing job. } \\
(I=\text { detrimental, 3 = no impact, 5 = helpful })\end{array}$ & 4.0 & 1.6 & 4.2 & 0.8 \\
\hline $\begin{array}{l}\text { Comfort in sharing separation responsibility. } \\
(I=\text { nol comfortable, 3 = moderately comfortable, } \\
5=\text { very comfortable })\end{array}$ & 4.2 & 1.2 & 4.5 & 0.8 \\
\hline
\end{tabular}

Table 26. Pilot Participant Mean Ratings of Separation Responsibility Confusion

\begin{tabular}{|l|c|c|c|c|c|c|c|c|}
\hline \multirow{2}{*}{ N=6 } & \multicolumn{2}{|c|}{ CO } & \multicolumn{2}{c|}{ CO:CDTI } & \multicolumn{2}{c|}{ SS:L1 } & \multicolumn{2}{c|}{ SS:L2 } \\
\cline { 2 - 8 } & M & SD & M & SD & M & SD & M & SD \\
\hline $\begin{array}{l}\text { Uncertainty of who } \\
\text { had separation } \\
\text { authority. }\end{array}$ & 1.2 & 0.4 & 1.0 & 0 & 1.8 & 1.0 & 1.0 & 0 \\
\hline
\end{tabular}

$(I=$ very low, $3=$ moderate, $5=$ very high $)$

\subsubsection{Pilot Information Requirements}

Following the simulation, pilot participants were asked several questions regarding the use and the appropriateness of the tools provided. Although the pilots usually detected the conflicts prior to the alerting, the data revealed that they found the CDTI and the associated conflict alerting logic quite effective for safe operations. The pilot participants also indicated that the amount of information on the CDTI-AL was adequate to identify and resolve conflicts and that the timing of the conflict alert was adequate for strategic separation tasks. Table 27 summarizes the pilots responses. Data from the Post-Run Forms found that pilot participants felt the timeliness of the CDTI-AL conflict alert was adequate across the conditions. Mean ratings and SDs are reported in Table 28. 
Table 27. Pilot Participant Mean Ratings of Information.

\begin{tabular}{|c|c|c|}
\hline N=6 & M & SD \\
\hline $\begin{array}{c}\text { Effectiveness of CDTI and alerting for safe operations. } \\
(I=\text { not effective, 3 = moderately effective, 5 = very effective })\end{array}$ & 4.8 & 0.4 \\
\hline $\begin{array}{l}\text { Amount of information on CDTI to identify and resolve conflicts. } \\
(I=100 \text { little, 3 = adequate, 5 = } 100 \text { much })\end{array}$ & 3.5 & 0.8 \\
\hline $\begin{array}{l}\text { Time CDTI alerting provided for strategic shared-separation. } \\
(I=\text { too little, 3 = adequate, 5 = too much })\end{array}$ & 3.3 & 0.5 \\
\hline
\end{tabular}

Table 28. Pilot Participant Mean Ratings of the Timeliness of CDTI-AL Alerts

\begin{tabular}{|c|cc|c|c|c|c|}
\hline \multirow{2}{*}{ N =6 } & \multicolumn{2}{|c|}{ CO:CDTI } & \multicolumn{2}{c|}{ SS:L1 } & \multicolumn{2}{c|}{ SS:L2 } \\
\cline { 2 - 7 } & $M$ & SD & $M$ & SD & $M$ & SD \\
\hline Timeliness of CDTI-AL alert & 3.3 & 0.5 & 3.0 & 0 & 3.0 & 0 \\
\hline
\end{tabular}

$(1=$ too early, $3=$ adequate, $5=$ too late $)$

Pilot form data indicated that flight crews spent a considerable amount of time monitoring the CDTI-AL with the average percentage of time increasing as the pilot responsibility for separation increased. Table 29 displays means and $S D$ s for monitoring time. Flight crews reported spending about two-thirds of the time monitoring the CDTI-AL, therefore, it is important to note that in addition to the CDTI-AL information, the Navigation Display served as an important data source for information on their current and programmed route of flight, weather, and other FMS information. However, concerns about spending too much time monitoring the CDTI were mentioned consistently by nearly all pilots involved in this study.

Table 29. Mean Percentage of Time Pilot Participants Spent Monitoring the CDTI-AL

\begin{tabular}{|l|c|c|c|c|c|c|}
\hline \multirow{2}{*}{$\mathrm{N}=6$} & \multicolumn{2}{|c|}{ CO:CDTI } & \multicolumn{2}{c|}{ SS:L1 } & \multicolumn{2}{c|}{ SS:L2 } \\
\cline { 2 - 7 } & $\mathrm{M}$ & SD & $\mathrm{M}$ & SD & $M$ & SD \\
\hline $\begin{array}{l}\text { Time spent monitoring } \\
\text { CDTI-AL }\end{array}$ & $62.5 \%$ & $20.9 \%$ & $66.7 \%$ & $21.4 \%$ & $68.3 \%$ & $21.1 \%$ \\
\hline
\end{tabular}


In order to investigate how the flight crews may have been using the time they spent monitoring the CDTI-AL, the researchers calculated how often flight crews used the various CDTI-AL functions provided in this study, including the temporal predictors, callsign/ground speed information and selectable map range levels. Of the 27 flight segments in which the CDTI-AL was available to the pilot participants, on average they had the temporal predictors selected "ON" during $89.7 \%(S D=14.4 \%)$ of the flight segment time. In addition, call sign/ground speed information was selected "ON" for an average of $13.4 \%(S D=14.6 \%)$ of the flight segment time.

Flight crews could also use the selectable map range functionality of the Navigation Display to help de-clutter their display. There were seven hard-coded map range values that the pilots could select from on the Boeing 747-400 Navigation Display (10, 20, 40, 80, 160, 320, and $640 \mathrm{~nm}$ ). Figure 29 depicts the average amount of flight segment time spent at each available Navigation Display range. Flight crews spent the majority of the time at the $160 \mathrm{~nm}$ range (around $55 \%$ of the time across conditions), followed by the $80 \mathrm{~nm}$ (roughly $35 \%$ of the time). This is consistent with the proposed ADS-B range of $120 \mathrm{~nm}$, as $160 \mathrm{~nm}$ selection provides the full extent of that range (RTCA, 1992).

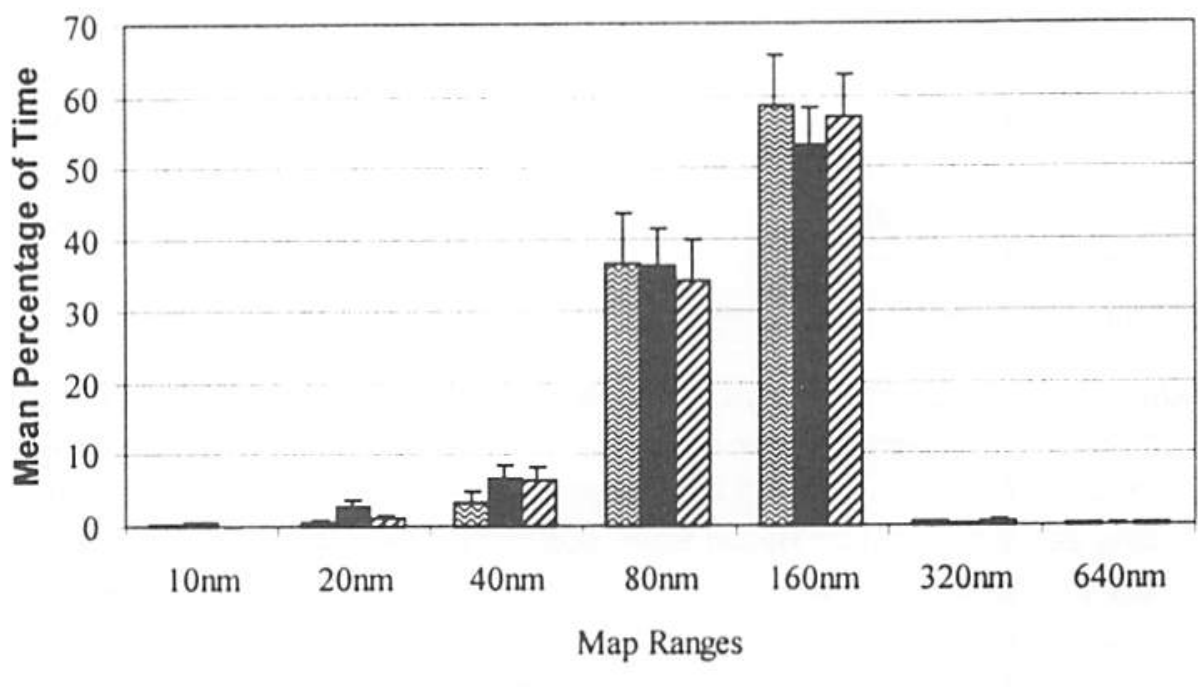

® $\mathrm{CO}: \mathrm{CDTl} \square \mathrm{SS}: \mathrm{L} 1 \square \mathrm{SS}: \mathrm{L} 2$

Figure 29. Mean percentage of time pilot participants spent at each map range level. 


\subsubsection{Pilot Workload and Situation Awareness}

\subsubsection{Pilot Workload}

- AHP Workload Ratings.

Pilot participants were asked to indicate which condition they considered better for reducing workload. Again they responded with a preference for SS:L2 over the other conditions Table 30 presents AHP factor loadings and level of dominance ratings. Pilots preferred SS:L2 over CO by a factor of 5.9, over CO:CDTI by a factor of 2.4 and over SS:L1 by a factor of 2.9. SS: $\mathrm{Ll}$ was preferred over $\mathrm{CO}$ by a factor of 2.7 but was equally preferred to CO:CDTI.

Table 30. Pilot Participant Preference for Reducing Workload

\begin{tabular}{|l|c|c|}
\hline Reducing Workload Comparison & Factor & Level of Dominance \\
\hline CO:CDTI preferred over CO & 2.5 & weak \\
\hline SS:L1 preferred over CO & 2.7 & weak \\
\hline SS:L2 preferred over CO & 5.9 & strong \\
\hline SS:L1 equal to CO:CDTI & 1.1 & equal \\
\hline SS:L2 preferred over CO:CDTI & 2.4 & weak \\
\hline SS:L2 preferred over SS:L1 & 2.9 & weak \\
\hline
\end{tabular}

- Subjective Workload Ratings

Pilot participants were asked to rate workload levels after the completion of each condition. Results are presented in Table 31. In general, pilot workload ratings were low, only ranging from very low to low. Regardless, inspection of the means indicates that the ratings are generally higher for SS:Ll when compared to the other conditions. 
Table 31. Pilot Participant Mean Workload Ratings

\begin{tabular}{|c|c|c|c|c|c|c|c|c|}
\hline \multirow{2}{*}{$N=6$} & \multicolumn{2}{|c|}{ CO } & \multicolumn{2}{c|}{ CO:CDTI } & \multicolumn{2}{c|}{ SS:L1 } & \multicolumn{2}{c|}{ SS:L2 } \\
\cline { 2 - 9 } & $M$ & $S D$ & $M$ & SD & $M$ & SD & $M$ & SD \\
\hline Physical & 1.0 & 0 & 1.0 & 0 & 1.8 & 0.8 & 1.5 & 0.6 \\
\hline Mental & 1.0 & 0 & 1.3 & 0.5 & 2.0 & 0.6 & 1.8 & 0.8 \\
\hline Overall & 1.0 & 0 & 1.3 & 0.5 & 1.8 & 0.4 & 1.5 & 0.6 \\
\hline Air $\leftrightarrow$ Ground Comm & 1.2 & 0.4 & 1.2 & 0.4 & 1.2 & 0.4 & 1.3 & 0.5 \\
\hline Crew Coordination & 1.2 & 0.4 & 1.0 & 0 & 1.5 & 0.6 & 1.2 & 0.4 \\
\hline Aircraft Separation & N/A & N/A & N/A & N/A & 2.0 & 0.6 & 1.8 & 0.8 \\
\hline Air $\leftrightarrow$ Air Comm. & N/A & N/A & N/A & N/A & 1.5 & 0.8 & 1.3 & 0.5 \\
\hline
\end{tabular}

$(I=$ very low, $3=$ moderate, $5=$ very high $)$

- Pilot Participant Situation Awareness Measures

Overall situation awareness was defined for the flight crews as "What is commonly known as the pilot's 'staying ahead of the aircraft' where the pilot has a thorough understanding of the current situation and can take appropriate action as necessary."

- AHP: Maintaining Situation Awareness.

Using the AHP, pilot participants rated which condition they considered better for maintaining situation awareness. Table 32 illustrates the flight crew's condition preferences. Pilots favored SS:L2 for maintaining situation awareness over the other conditions. They preferred SS:L2 over CO for maintaining situation awareness by a factor of 9.9 , over CO:CDTI by a factor of 3.3 and over SS:Ll by a factor of 2.1. SS:Ll was preferred over CO by a factor of 4.7 but was rated equal to CO:CDTI for this question. Predictably, pilots reported that having a CDTI-AL (CO:CDTI) may have helped with situation awareness as CO:CDTI was preferred over CO by a factor of 3.0 . 
Table 32. Pilot Participant Preference for Maintaining Situation Awareness

\begin{tabular}{|l|c|c|}
\hline \multicolumn{1}{|c|}{$\begin{array}{c}\text { Maintaining Situation Awareness } \\
\text { Comparison }\end{array}$} & Factor & Level of dominance \\
\hline CO:CDTI preferred over CO & 3.0 & weak \\
\hline SS:L1 preferred over CO & 4.7 & $\begin{array}{c}\text { between strong and weak } \\
\text { (closer to strong) }\end{array}$ \\
\hline SS:L2 preferred over CO & 9.9 & absolute \\
\hline SS:L1 equal to CO:CDTI & 1.6 & equal \\
\hline SS:L2 preferred over CO:CDTI & 3.3 & weak \\
\hline SS:L2 preferred over SS:L1 & 2.1 & weak \\
\hline
\end{tabular}

\subsection{Integrated Results}

The data in the following section discuss a subset of the total data obtained from this study. This section contains the data that were comparable between the controller and pilot participants.

\subsubsection{Operational Issues that Affect Shared-Separation Operations}

\subsubsection{Safety Measures}

- Loss of Separation for Conflicts Involving the NASA ARC Simulator.

In all conditions, the minimum separation distance was defined as either five nm horizontally or $1000 / 2000 \mathrm{ft}$ vertically (as appropriate). Pilot participants did not violate minimum separation standards or receive any TCAS alerts in any flight segment of any condition.

- Loss of Separation for Conflicts Involving WJHTC Simulation Pilots.

During the simulation there were two losses of separation involving WJHTC simulation pilots. The two separation violations occurred in SS:L2. One violation involved a planned conflict that resulted in a MSD of $4.9 \mathrm{~nm}$ while the aircraft were at the same altitude. The other was an unplanned conflict with a MSD of $4.0 \mathrm{~nm}$ while the aircraft were separated by only $900 \mathrm{ft}$. It must be noted that controllers were not allowed to cancel free flight in SS:L2. Both separation losses were determined to be due to late maneuvering (script execution error) by WJHTC simulation pilots. In conclusion there was no clear relationship between the losses of separation and shared-separation operations. 
- Subjective Ratings related to Safety.

Following each run, the pilot and controller participants were asked to rate the level of safety using that set of procedures and tools compared to current flight operations. $\mathrm{CO}$ was identical to current operations, so there are no data for this condition. See Figure 30 for mean and \pm 1 SEM bars for the various conditions. Inspection of the means suggest that the pilots felt that safety was somewhat enhanced with the addition of the new aircraft technologies and/or shared-separation procedures. The controllers, however, rated the level of safety as lower for the two shared-separation conditions (SS:L1 and SS:L2) when compared to their ratings of $\mathrm{CO}: \mathrm{CDTI}$. In addition, controller ratings were lower than pilot ratings in all conditions.

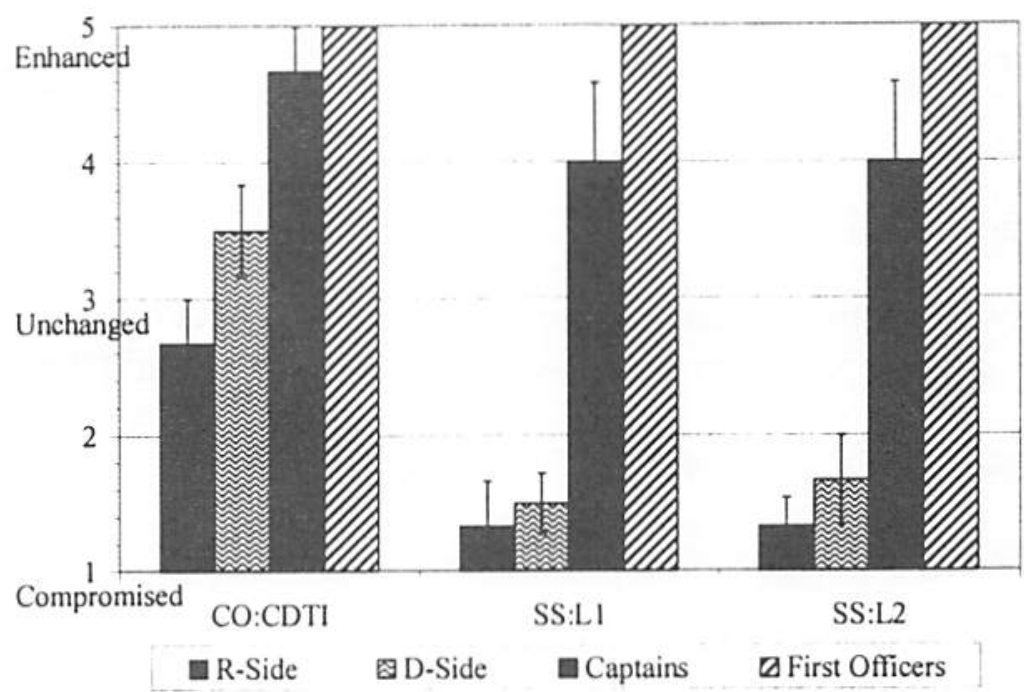

Figure 30. Controller and pilot participant mean ratings of safety.

\subsubsection{Aircraft Maneuver Strategies for Conflict Resolution}

- Aircraft Maneuver timing

For the flight crew participants, aircraft maneuver time was calculated for the two conditions in which the NASA ARC simulator had the freedom to maneuver independent of ATC (SS:L1 and SS:L2). For these two conditions, aircraft maneuver timing was measured as the duration from the beginning of the flight segment until the NASA ARC simulator initiated the first conflict avoidance maneuver. Table 33 shows means and $S D$ s for aircraft maneuvering start times. Across the shared-separation conditions, pilot participants started maneuvering to avoid conflicting aircraft on average less than 5 minutes into each flight segment, which was still roughly 2 to 3 minutes before the CDTI-AL would have indicated a pending alert. The data suggest that the different sets of pilot procedures did not impact the timing of aircraft resolution maneuvers. 
Table 33. Aircraft Maneuvering Mean Start Times During Shared-Separation

\begin{tabular}{|l|c|c|c|c|}
\hline \multirow{2}{*}{ N=6 } & \multicolumn{2}{|c|}{ SS:L1 } & \multicolumn{2}{c|}{ SS:L2 } \\
\cline { 2 - 5 } & $M$ & SD & $M$ & SD \\
\hline $\begin{array}{l}\text { Aircraft Maneuver } \\
\text { Times (min:sec) }\end{array}$ & $4: 48$ & $2: 05$ & $4: 29$ & $2: 59$ \\
\hline
\end{tabular}

Because flight crews and controllers identified conflicts quickly and initiated resolution maneuvers, CDTI-AL alerts were not triggered for most of the flight segments. Table 34 presents the frequency of CDTI-AL alerts in relation to resolution maneuvers. There were two instances when flight crews received CDTI-AL alerts prior to their first resolution maneuver (once in SS:Ll, and once in SS:L2). In comparison, the flight crews received CDTI-AL alerts after they had initiated a resolution maneuver once in SS:L2, four times in SS:Ll, and once in CO:CDTI. These alerts suggest that the maneuvers the pilots initially enacted were not sufficient to resolve the conflict.

Table 34. Frequency of CDTI-AL Alerts in Relation to Maneuver Start Times

\begin{tabular}{|l|c|c|c|}
\hline Condition & $\begin{array}{c}\text { CDTI-AL Alerts Before } \\
\text { First Maneuver }\end{array}$ & $\begin{array}{c}\text { CDTI-AL Alerts After } \\
\text { First Maneuver }\end{array}$ & No CDTI-AL Alerts \\
\hline CO:CDTI & 0 & 1 & 8 \\
\hline SS:L1 & 1 & 4 & 4 \\
\hline SS:L2 & 1 & 1 & 7 \\
\hline
\end{tabular}

By design, in SS:L1, the NASA ARC simulator could have been under direct air traffic control (if the controller had canceled free flight) or under shared-separation procedures. Within SS:L1, there were four flight segments in which the NASA ARC simulator received a CDTI-AL alert after the flight crew had recognized the conflict and initiated a resolution maneuver. In three of these instances, the controllers did not cancel free flight, and the flight crew retained separation responsibility until the conflict was resolved (as predicted by the aircraft alerting logic). Interestingly, in one of those four SS:L1 flight segments, the controller did cancel free flight but not until after the flight crew had initiated a maneuver that - according to the logic - was sufficient to resolve the conflict. In the one case in which the CDTI-AL alert triggered before maneuvering, the controller canceled free flight before the flight crew took any action. 
- Aircraft Maneuver Types.

In each flight segment, the pilot participants and the controllers could use heading, speed, altitude, or any combination thereof to resolve conflicts. Table 35 details the frequency of maneuver types issued by controllers to either the pilots and/or the intruder simulation pilot. Table 36 lists the frequency of maneuver type initiated by the pilots in the two sharedseparation conditions.

Table 35. Frequency and Type of Maneuvers Issued by Controllers to Resolve Conflicts Between the Pilot Participants and the Intruder Simulation Pilot

\begin{tabular}{|l|c|c|c|}
\hline \multirow{2}{*}{ Condition } & \multicolumn{3}{|c|}{$\begin{array}{c}\text { Controller Issued Maneuvers to Pilot Participants and/or } \\
\text { Intruder Simulation Pilot }\end{array}$} \\
\cline { 2 - 4 } & Heading & Altitude & Speed \\
\hline CO & 3 & 6 & 0 \\
\hline CO:CDTI & 11 & 1 & 0 \\
\hline SS:L1 & 4 & 1 & 1 \\
\hline SS:L2 & \multicolumn{2}{|c|}{ Controllers could not cancel shared-separation procedures in this condition } \\
\hline
\end{tabular}

Table 36. Frequency and Type of Maneuvers Initiated by Pilot Participants and/or Intruder Simulation Pilot to Resolve Conflicts

\begin{tabular}{|l|c|c|c|}
\hline \multirow{2}{*}{ Condition } & \multicolumn{3}{|c|}{$\begin{array}{c}\text { Maneuvers Initiated by the Pilot Participants and/or } \\
\text { Intruder Simulation Pilot to Resolve Conflicts }\end{array}$} \\
\cline { 2 - 4 } & Heading & Altitude & Speed \\
\hline CO & \multicolumn{3}{|c|}{ Flight crews could not initiate maneuvers in this condition } \\
\hline CO:CDTI & \multicolumn{2}{|c|}{ Flight crews could not initiale maneuvers in this condition } \\
\hline SS:L1 & 7 & 1 & 4 \\
\hline SS:L2 & 8 & 1 & 5 \\
\hline
\end{tabular}

Interestingly, in SS:L1, controllers cancelled free flight in five of the nine flight segments. They then instructed either the pilot participants or the intruder simulation pilot to implement heading and speed maneuvers larger than those that had been chosen by the flight crews. 
Participants (controllers and/or pilots) often used multiple sub-maneuvers to resolve a conflict. The multiple conflict resolution maneuvers were typically enacted sequentially to resolve a single conflict. For example, a flight crew would begin by using a heading change, and then add an altitude maneuver when it appeared that the heading change would not provide adequate aircraft separation. Table 37 represents the combination of maneuvers used by pilots and/or controllers for conflict resolutions.

Table 37. Maneuvers Used by Controllers and/or Pilot Participants to Resolve Conflicts Involving the NASA ARC Simulator

\begin{tabular}{|l|c|c|c|c|}
\hline Maneuver & CO & CO:CDTI & SS:L1 & SS:L2 \\
\hline Speed only & - & - & - & - \\
\hline Heading only & 3 & 8 & 3 & 4 \\
\hline Altitude only & 6 & 1 & - & - \\
\hline Speed + heading & - & - & 4 & 4 \\
\hline Speed + altitude & - & - & - & 1 \\
\hline Heading + altitude & - & - & 1 & - \\
\hline Speed + heading + altitude & - & - & 1 & - \\
\hline
\end{tabular}

For those flight segments that did not have an altitude change included in the resolution maneuver, the horizontal distances between the NASA ARC simulator and intruder aircraft at the MSD was calculated. Table 38 provides descriptive statistics for the MSD data. This allowed us to investigate if there may be differences between pilots and controllers in how much distance between aircraft each maintained. Inspection of the means for SS:L1 and SS:L2 suggest that the flight crews tended to achieve less horizontal separation than when the controller teams were providing separation in $\mathrm{CO}$ and $\mathrm{CO}: \mathrm{CDTI}$. It is difficult to interpret the data for the SS:Ll conflicts when free flight was canceled since these results are influenced by the fact that both the pilots and controllers worked to resolve the conflicts. 
Table 38. Descriptive Statistics for Minimum Horizontal Distance for Conflicts Involving NASA ARC Simulator (Resolved with Horizontal Separation Only)

\begin{tabular}{|c|c|c|c|}
\hline Condition & $\begin{array}{c}\text { Pilot (only) Resolved } \\
\text { Conflicts }\end{array}$ & $\begin{array}{c}\text { Free Flight Cancelled } \\
\text { Conflicts } \\
\end{array}$ & $\begin{array}{l}\text { Controller (only) } \\
\text { Resolved Conflicts }\end{array}$ \\
\hline $\mathrm{CO}$ & $\begin{array}{l}\text { Flight crews could not initiate } \\
\text { maneuvers in this condition. }\end{array}$ & Not Applicable & $\begin{aligned} \text { Mean } & =10.5 \\
S D & =3.5 \\
\mathrm{~N} & =4\end{aligned}$ \\
\hline $\mathrm{CO}: \mathrm{CDTI}$ & $\begin{array}{l}\text { Flight crews could not initiale } \\
\text { maneuvers in this condition. }\end{array}$ & Not Applicable & $\begin{array}{c}\text { Mean }=11.0 \\
S D=2.8 \\
\mathrm{~N}=8\end{array}$ \\
\hline SS:Ll & $\begin{array}{c}\text { Mean }=8.7 \\
S D=5.7 \\
\mathrm{~N}=3\end{array}$ & $\begin{array}{c}\text { Mean }=8.2 \\
S D=3.4 \\
\mathrm{~N}=4\end{array}$ & $\begin{array}{l}\text { Controller involvement in } \\
\text { confict resolutions for this } \\
\text { condition was the result of frec } \\
\text { fight cancellations. }\end{array}$ \\
\hline SS:L2 & $\begin{array}{c}\text { Mean }=6.2 \mathrm{~nm} \\
S D=1.1 \mathrm{~nm} \\
\mathrm{~N}=8\end{array}$ & $\begin{array}{l}\text { There were no free fight } \\
\text { cancellations by fight crews. } \\
\text { Conirollers could not cancel } \\
\text { free flight in this condition. }\end{array}$ & $\begin{array}{l}\text { There were no free fight } \\
\text { cancellations by fight crews. } \\
\text { Controllers could not cancel } \\
\text { free flight in this condition }\end{array}$ \\
\hline
\end{tabular}

- Air $\leftrightarrow$ Ground Communication

In each of the conditions, an air $\leftrightarrow$ ground frequency was available for all flight crews to contact controllers or to receive instructions and advisories from ATC. All flight crews conducted standard ATC communications, such as initial sector check-ins, and any free flight related communications on the same frequency. Figure 31 depicts the mean frequency of air $\leftrightarrow$ ground transactions between the pilot and controller participants. An air $\leftrightarrow$ ground transaction was defined as all communication initiated by a controller or pilot participant. For example, a transaction could be all verbalizations made by the controllers and pilots during the transfer of communication exchange or all comments made about what maneuver the flight crew was making to resolve a CDTI-AL alert. It was not necessary that a transaction have an acknowledgment; however, if there was an acknowledgment, it was counted as part of the same transaction. The frequency was a summation of all the transactions between pilot and controller participants during the three flight segments for each condition. The mean frequency for each condition was then calculated as the average of the three flight crew frequencies. The data suggest that there were more air $\leftrightarrow$ ground 


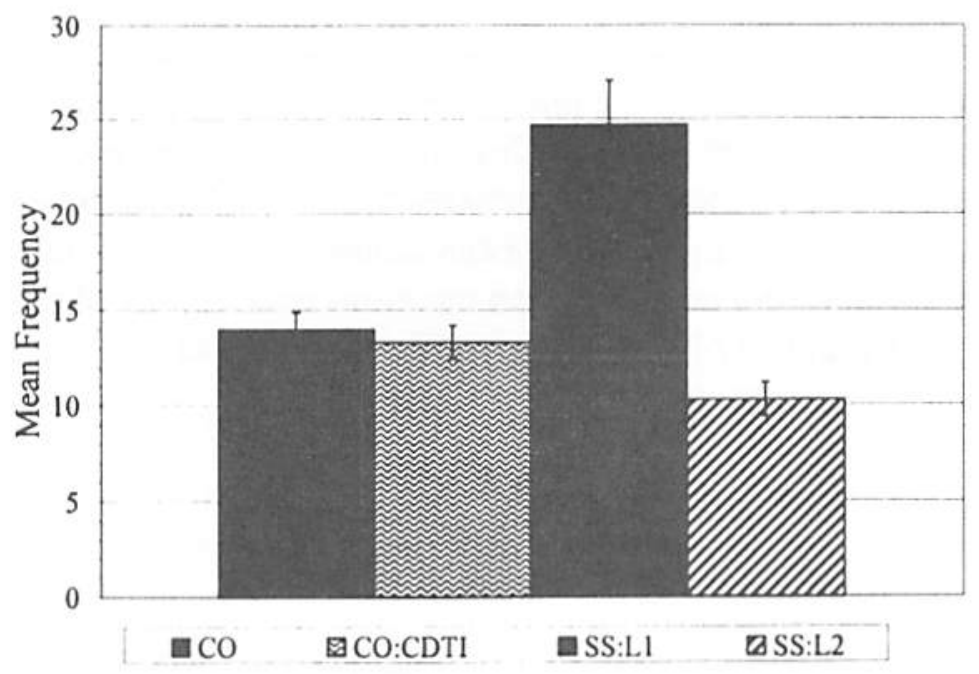

Figure 31. Mean frequency of air $\leftrightarrow$ ground transactions.

transactions in SS:L1 compared to the other three conditions. Compared to SS:L1, there were $42 \%$ fewer transactions in SS:L2, $54.8 \%$ fewer transactions in CO:CDTI, and $57 \%$ fewer transactions in CO. This finding may be explained by the fact that in SS:L1, all flight crews were instructed to inform the controllers of all conflict avoidance maneuvers they were making prior to initiating them.

All flight crews were asked to inform the controllers prior to the execution of any and all of their aircraft maneuvers in this condition. The procedural requirement to inform the controllers in SS:L1 was also examined as part of the air $\leftrightarrow$ ground communications. The data for SS:L1 indicated that within each of these nine flight segments, the pilot participants always notified the controllers of maneuver changes (e.g., heading or speed change) at least once. For example, if speed changes were made for conflict resolution more than once in a flight segment, the pilots always notified the controller at least one time that speed was being modified. In addition, the number of sub-maneuvers made by the NASA ARC simulator when avoiding the intruder aircraft was tallied. Some of these sub-maneuvers were considered minor adjustments, particularly in these fairly short flight segments, therefore only those of a relevant magnitude were analyzed (speed changes 10 knots or greater, heading changes five degrees or greater, and any altitude change). These data indicate that the condition where flight crews were required to inform controllers of their intent, in addition to informing at least once, pilot participants also informed controllers about several of their sub-maneuvers. They did so for 28 out of 39 sub-maneuvers. All of these notifications to the controllers occurred either just prior to the execution of the sub-maneuver or just as they began the sub-maneuver. In SS:L2, the pilots were instructed that informing the controller of their aircraft maneuvers was voluntary. The data revealed that for SS:L2, the pilots notified the controller of their intent in only 1 out of 37 sub-maneuvers used to avoid the intruder aircraft.

In addition to the number of air $\leftrightarrow$ ground communications, the average air $\leftrightarrow$ ground transaction duration was calculated. The total transaction time was measured from the beginning of the first instruction, question, or comment made by any of the controller or pilot 
participants to the end of the final communication on the topic. This was meant to represent the time it would take to handle a complete transaction because it is common practice to not interrupt an ongoing ATC exchange. This time is meant to represent the total time required to complete a transaction, therefore it included the brief silences between pilot and controller communications. Figure 32 shows the mean transaction duration (and \pm 1 SEM bars) for each condition. Mean air $\leftrightarrow$ ground transaction durations appear to be shorter in SS:L2 when compared to SS:L1 and CO:CDTI but not different from CO.

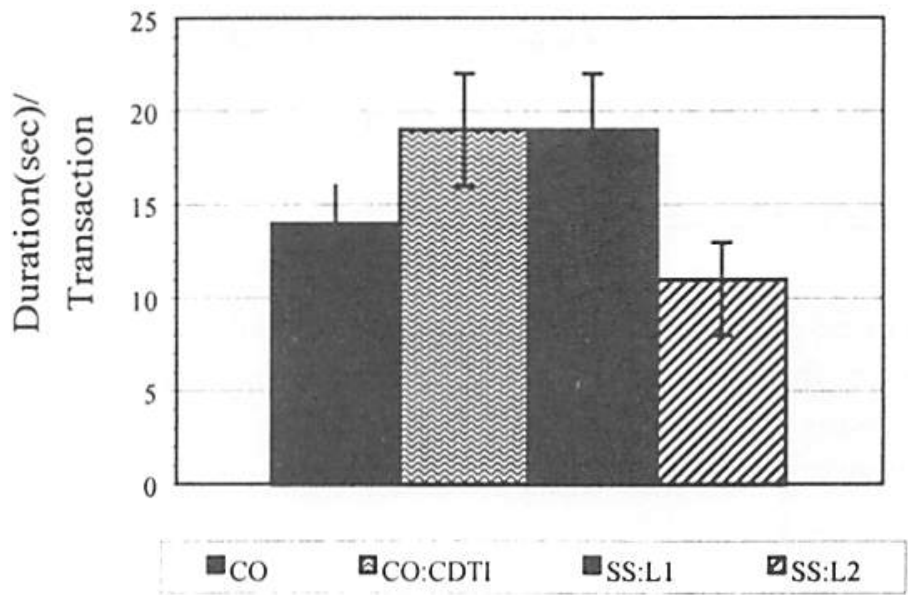

Figure 32. Mean duration of air $\leftrightarrow$ ground transactions.

The number of missed communications was also investigated. This included any communication between the pilot and the controller participants, for which there was no response, excluding events that are associated with the flight crew being on the wrong ATC frequency. In $\mathrm{CO}$, there were 39 communication events. The flight crews missed one of these communications $(2.6 \%)$, and there were no missed communications by the controllers. In CO:CDTI, the pilot participants missed one communication out of $40(2.5 \%)$ events. SS:L1 had a total of nine communications out of $74(5 \%)$, with five communications missed by the flight crews and four by the controllers. It is important to note that seven of these came from one flight crew/controller pairing. All of these missed communications were preceded by taskrelated comments (i.e., instructions, notifications, or queries), with the exception of one call in which the controller asked the pilot participants if "they had time for a question" (for which they received no response). None of the 35 communications in SS:L2 were missed.

\subsubsection{Information Requirements and Procedures}

\subsubsection{Cancellation of Free Flight Operations}

In SS:L1, controllers were instructed they could cancel free flight for one or a pair of aircraft at a time. During the nine NASA ARC simulator flight segments in this condition (three repetitions 
for each of three flight crews), the controller team canceled free flight in five (56\%) of the runs. In all five runs, the NASA ARC simulator and intruder aircraft were at the same altitude, and the flight crews were using heading and/or speed to attempt to resolve the conflict. The mean horizontal distance between conflicting aircraft at the time of free flight cancellation was 13.1 $\mathrm{nm}(S D=4.6)$. Horizontal distances between conflicting aircraft ranged from $9.0 \mathrm{~nm}$ to $19.9 \mathrm{~nm}$.

In the cases in which free flight was canceled, the data were examined to determine the maneuvering strategies for the controllers and pilot participants. In four out of five of the cancellations in SS:L1, the controllers issued an instruction for the same type of maneuver, but of a greater magnitude than the flight crew had already begun. For example, if the flight crew had started a turn to relieve the conflict, the controller cancelled free flight and instructed the flight crew to turn more sharply. In the fifth instance, the flight crew had attempted to use speed to resolve the conflict, and the controller followed the cancellation with an instruction to change altitude. In four out of the five flight segments, the controller returned the separation responsibility to the aircraft once the conflict had been resolved.

All pilots could cancel free flight operations at any time in either of the shared-separation conditions (SS:L1, SS:L2). However, neither the simulation pilots (per simulation design) nor the pilot participants requested intervention from the controllers.

\subsubsection{Subjective Ratings related to Procedures}

Figure 33 shows controller and pilot participant ratings for the time available to assure safe aircraft separation. Pilot participants did not conduct separation tasks in CO or CO:CDTI, therefore they were not asked this question for those conditions. In general, the pilots rated the time available for this task as adequate. Controller mean ratings for CO:CDTI appear to be slightly higher than their mean ratings for SS:L1 and SS:L2.

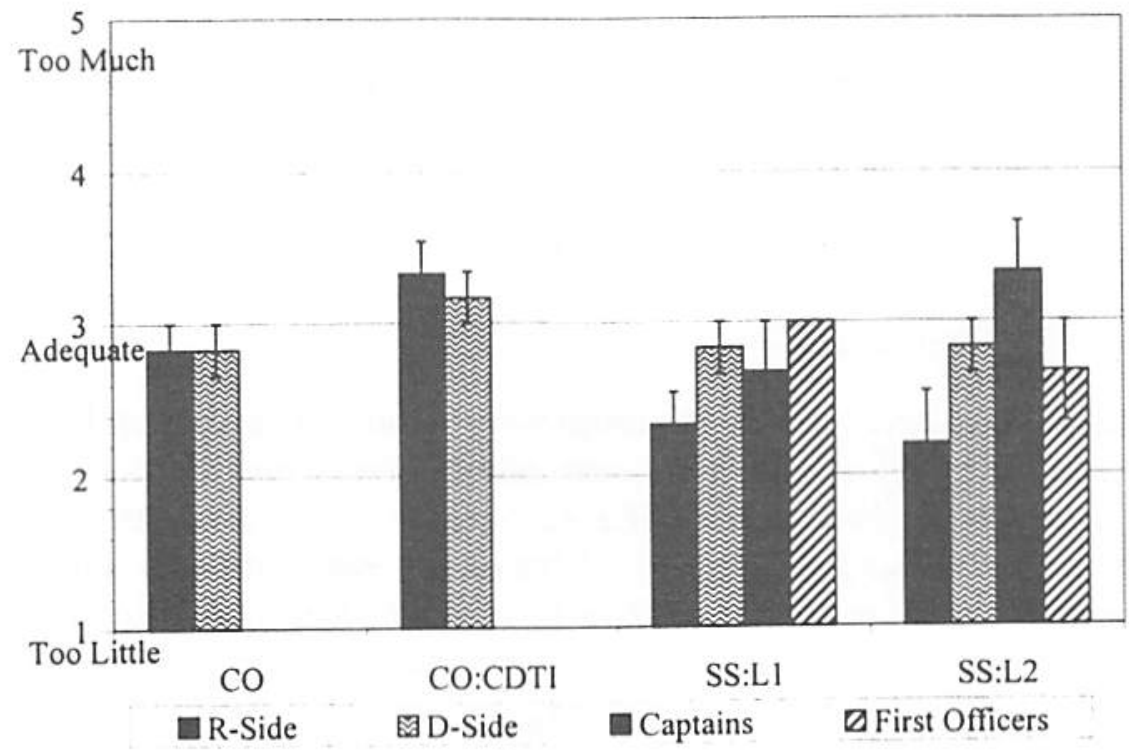

Figure 33. Controller and pilot participant mean ratings for time available to assure safe separation. 
Figure 34 shows the controller and pilot participant ratings for the amount of time available for coordination and communication tasks. These tasks are likely to be at least partially dependent on when the automation tools detect the conflict. CDTI-AL provided conflict alerts about 7 minutes prior to potential loss of separation for the pilots. URET provided conflict alerts about 13-17 minutes prior to potential loss of separation for the controllers. Therefore, one may have expected to see some differences between the pilots and controllers. However, inspection of the means reveals that there were little differences between the four conditions for the time available to complete required coordination and communication. The time allowed was typically rated as adequate by all participants with the exception that the captains' rating for CO does appear to be higher, indicating that there was more than adequate time for the communication and coordination events.

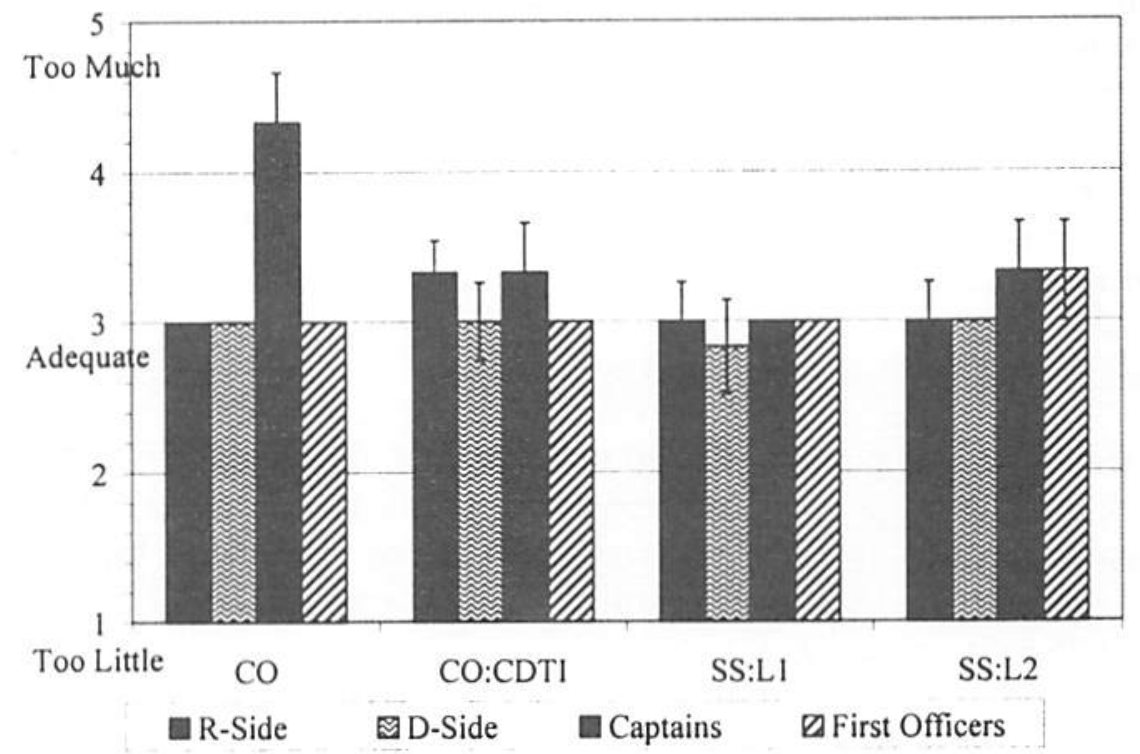

Figure 34. Controller and pilot participant mean ratings for time for coordination and communication.

\subsubsection{Controller and Pilot Workload and Situation Awareness}

\subsubsection{Controller and Pilot Workload}

Finally, in addition to the forms, workload ratings were gathered from both pilots and controllers throughout the conditions. Pilot participants were asked to rate their workload for the flight at the end of each 20-minute flight segment within each condition. Controllers were asked to rate their workload every 5 minutes during their 1.5 hour run for each condition (previously referred to as controller interval workload data). In order to compare ratings over common flight segments, controller interval workload ratings corresponding to the pilot ratings were obtained by taking the average of the 5-minute ratings during the time that the NASA ARC simulator was on the target sector's radio frequency. The mean ratings for the pilot workload responses indicated a somewhat higher workload in SS:L1 and SS:L2 compared to pilot responses in CO. The corresponding average controller ratings seemed not to differ among the various conditions. However, the controller ratings were generally higher than the pilot ratings in CO and CO:CDTI, 
and similar in the shared-separation conditions. Again, pilot and controller participant workload ratings were rather low (see Figure 35).

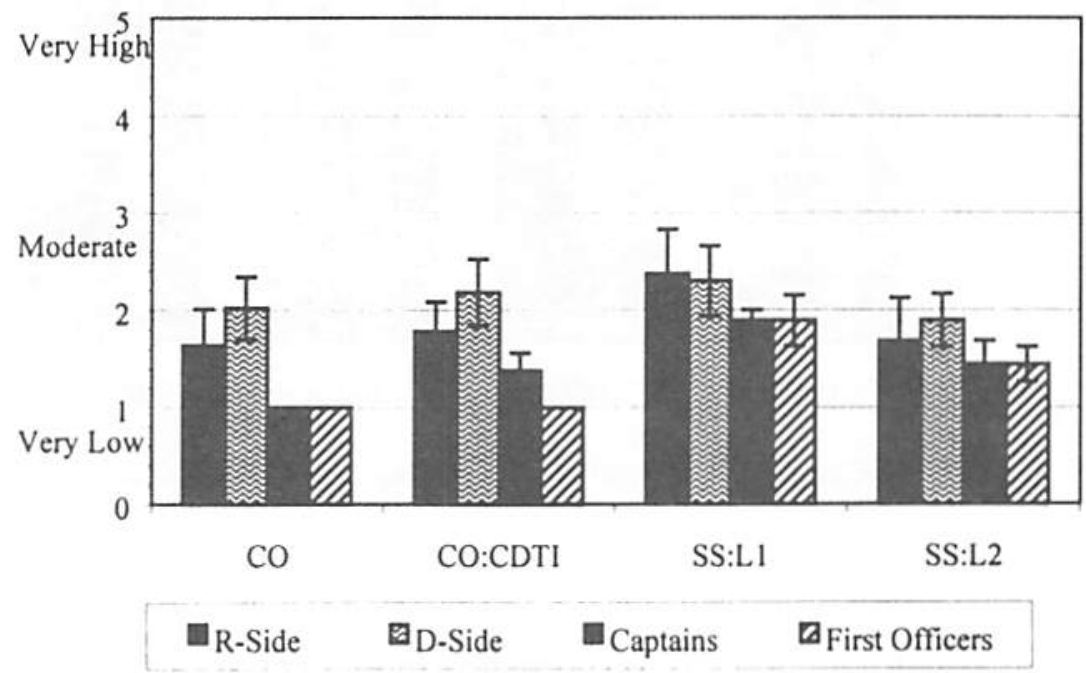

Figure 35. Controller and pilot participant mean workload ratings.

\subsubsection{Controller and Pilot Participant Situation Awareness}

Figure 36 shows controller and pilot participant ratings for the level of overall situation awareness for the four conditions. In general, controllers rated their overall situation awareness as high, and there were only small differences between the four conditions. Although there was a large amount of variance in the flight crew data, inspection of the captain and first officer means indicate that the pilots may perceive the shared-separation conditions as providing more situation awareness when compared to $\mathrm{CO}$ and $\mathrm{CO}$ :CDTI. 


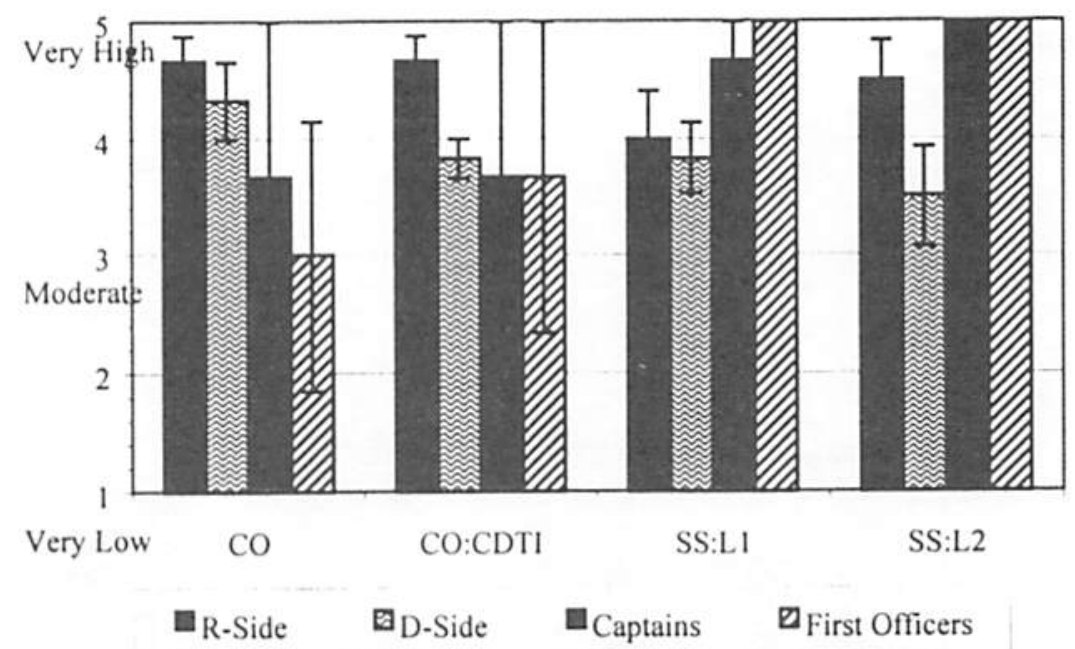

Figure 36. Controller and pilot participant mean ratings for overall situation awareness.

\section{DISCUSSION}

Within each sub-section, the results are discussed in the order corresponding to the three primary objectives of the study.

\subsection{Ground-Side Discussion}

\subsubsection{Operational Issues that Affect Shared-Separation Operations}

The results indicated that controllers rated the safety for procedures compared to current operations as compromised in SS:L1 and SS:L2. In their post-run ratings and comments, controllers expressed concern for safety while operating under shared-separation conditions. Controllers frequently commented that pilots waited too long to resolve aircraft conflicts and, from the controller perspective, pilot maneuvers were often barely adequate to ensure separation. Controllers also commented that pilots elected to fly their aircraft much closer to conflicting aircraft than controllers would normally allow.

The average duration of URET red and yellow alerts were longer for SS:L2 compared to the other conditions. These results for the URET red and yellow alerts were consistent with controller comments about pilots resolving conflicts later than controllers. In CO and CO:CDTI, controllers could clear a URET alert by resolving the conflict. In SS:L1, controllers could address a URET alert by first canceling free flight and then resolving the conflict, although they allowed many pilots to continue on free flight and solve their own conflicts. Both red and yellow alerts were longer in SS:L2 most likely because controllers were not allowed to cancel free flight and also because pilot participants as well as scripted simulation pilot maneuvers resolved conflicts later than controllers would have. Several controllers commented that not being able to resolve conflicts in a timely manner on their own terms was stressful. 
Although controllers often stated they felt safety was compromised in shared-separation operations, they rated the amount of time available to assure safe aircraft separation and complete required coordination as generally adequate in all four conditions. However, controller ratings indicated there was slightly more time available to assure safe aircraft separation for CO:CDTI compared to SS:L1 and SS:L2. In CO:CDTI (and CO), controllers tended to resolve aircraft conflicts early, whereas conflicts tended to be resolved later in SS:L1 and SS:L2. When aircraft conflicts were resolved relatively later, controllers may have felt that there was slightly less time available to assure safe aircraft separation.

The MSDs for altitude-resolved planned conflicts involving simulated aircraft flown by WJHTC simulation pilots were much greater for $\mathrm{CO}$ and $\mathrm{CO}: \mathrm{CDTl}$ compared to SS:L1 and SS:L2. The MSDs for vector-resolved conflicts, however, were not very different between the four conditions. The different results for altitude-resolved and vector-resolved conflicts were likely due to the different methods for computing the MSDs. It is important to understand that the MSD for an altitude-resolved conflict did not represent the absolute closest horizontal distance between the conflicting aircraft. The MSD represents only the horizontal distance when the aircraft pair have not achieved altitude separation. Most altitude-resolved conflicts passed very close to each other horizontally, but altitude ensured safe separation. In contrast, the MSD for a vector-resolved conflict represents the absolute closest horizontal distance between the conflicting aircraft. In the case of altitude-resolved conflicts, early actions produced greater MSDs relative to later actions. Therefore, the MSD results for altitude-resolved conflicts were consistent with the controller strategy to resolve conflicts early and allow a greater separation distance when controlling aircraft on their own terms.

Another indication that controllers felt that safety was compromised during shared-separation operations was they cancelled free flight for 12 of the $39 \mathrm{SS}: \mathrm{L} 1$ planned conflicts (31\%) involving WJHTC simulation pilots (all aircraft other than the NASA ARC simulator and intruder aircraft). In some cases, controllers cancelled free flight after one of the pilots initiated a conflict-resolving maneuver suggesting that controllers were not always certain that pilot maneuvers would be effective.

\subsubsection{Controller Information Requirements and Procedures}

Controllers rated the amount of information necessary to resolve conflicts and the look-ahead time of the URET conflict alerts as adequate in all four conditions. However, some controllers commented that URET alerts were too early in some cases and too late in others. In general, controllers indicated that URET was beneficial and provided enough information to help them identify and resolve aircraft conflicts in both current operational conditions and sharedseparation conditions. Although controllers felt that they had enough information to resolve conflicts, many controllers commented that they needed pilot intent information sooner during shared-separation operations.

Controllers rated the procedures for SS:L1 and SS:L2 as generally not helpful for performing their jobs. Controller comments focused on perceived reductions in safety. Controllers reported that they did not feel completely "in control" of the traffic situation, and this was very stressful. Controllers stated they felt that shared-separation procedures put them in a "reactive" control mode instead of allowing them to be "proactive" and use their typical planning skills. Other 
investigators had similar findings (Corker et al., 2001). Controllers were concerned that pilots would cancel free flight in a conflict situation that would be impossible for controllers to resolve in time. Controllers stated they felt that the pilot style for resolving conflicts compromised safety. As previously stated, controllers felt that pilots waited too long to resolve conflicts and maneuvered too closely to conflicting aircraft.

Controllers formed more URET trial plans in CO and CO:CDTI compared to SS:L1 and SS:L2. Controllers use the URET trial-planning feature as a tactical tool to help determine if a proposed control action would impact other aircraft. In CO and CO:CDTI, controllers were completely responsible for aircraft separation, which was likely why controllers frequently used trial planning. In SS:Ll, controllers may have used trial planning less because procedures allowed pilots maneuvering flexibility and thereby reduced time for controllers to plan and resolve conflicts (including trial planning) if/when they canceled free flight. In addition, during SS:L1 and SS:L2, controllers reported that they did not feel completely "in control" of the traffic situation. In SS:L2 especially, controllers may have felt that trial planning was not very useful because they were not able to cancel free flight and resolve aircraft conflicts. These changes in their roles and responsibilities may also explain why controllers used trial planning less during shared-separation operations.

In the initial study briefing, the researchers explained that controllers may monitor air $\leftrightarrow$ air communications in SS:L1 and SS:L2 as little or as much as they wanted. The researchers suggested that controllers should try monitoring during the training runs to determine if it was useful during shared-separation operations. Each R-side/D-side controller team decided that the D-side controller should monitor the air $\leftrightarrow$ air frequency. D-side controllers reported that they always monitored air $\leftrightarrow$ air communications. For half the teams, the R-side controller also decided to monitor air $\leftrightarrow$ air communications and reported that they always monitored as well. The R-side and D-side controllers who monitored the air $\leftrightarrow$ air frequency reported that it was useful. The R-side controllers who decided not to monitor the air $\leftrightarrow$ air frequency commented that it was distracting to listen to both air $\leftrightarrow$ air and air $\leftrightarrow$ ground communications at the same time, particularly during SS:Ll. These R-side controllers stated that it was sufficient that their $D$-side team member monitor air $\leftrightarrow$ air communications and report to them any necessary information. Additionally, controllers suggested that adding a speaker for air $\leftrightarrow$ air communications may have been helpful.

\subsubsection{Controller Workload and Situation Awareness}

Several different measures and techniques in the study assessed controller workload. The researchers employed a form to collect post-run workload ratings and the WAK tool to collect interval workload ratings as controllers worked the traffic. In addition, experienced air traffic control specialists participated as EOs in the simulation and provided ratings of controller physical taskload, a measure related to workload. Finally, controller workload was a major topic of discussion in the post-run and exit debriefings.

The results indicated that controller post-run ratings of mental and overall workload were higher in SS:Ll compared to the other conditions. Controllers commented that having to monitor aircraft conflicts that they would have resolved earlier added a great deal to their mental workload and increased their stress level. Controllers reported that under current operating 
procedures, they routinely resolve conflicts early and then they do not have to think about the conflicts any further. However, shared-separation operations seemed to change their monitoring style and forced them to keep more situations in mind than they do normally. Additionally, controllers repeatedly stated that having to develop multiple alternative plans depending upon what pilots might do increased their workload. Controller ratings indicated that most of the additional workload of shared-separation operations was mental, and there was not much more physical workload.

Although controllers reported that mental and overall workload were higher in SS:L1, their ratings indicated that their absolute workload levels were not much above moderate. The traffic scenarios developed for the simulation were not very complex compared to actual traffic in these two sectors. By design, the simulation depicted rather sterile traffic situations with very few transitioning aircraft climbing or descending into adjacent airspace. Additionally, mixed equipage was not addressed; there were no restrictions due to active SUA or in-trail requirements, and no adverse weather conditions were in effect. Overall, the traffic scenarios were moderately busy, had lower complexity, and did not cause high workload for controllers.

Controller post-run workload ratings were generally the same or lower in SS:L2 compared to SS:L1. In one regard, controller workload might be expected to increase in SS:L2 because controllers did not receive pilot intentions of their maneuvers. However, there were critical differences between SS:L1 and SS:L2 that may have reduced controller workload. In contrast to SS:L1, there were no free flight cancellations in SS:L2 because controllers were not allowed to cancel free flight and no pilots requested cancellation. Controllers were not permitted to deliver any control instructions in SS:L2, therefore there were fewer communications to pilots and controllers reported that they only sometimes evaluated alternative plans to assure aircraft separation. These artifacts may have resulted in lower controller workload for SS:L2.

The interval workload ratings collected using the WAK tool were slightly different from the controller post-run workload ratings. In general, the interval workload ratings did not indicate any significant differences between the four conditions. The differences in workload for the interval and post-run workload rating techniques may be because the techniques rely on slightly different workload information (instantaneous versus overall). EO ratings of controller physical taskload were consistent with the results of the controller post-run workload ratings and controller comments about higher workload in SS:L1.

Controllers made fewer ground $\rightarrow$ air and land line PTTs in SS:L2 compared to the other conditions. A content analysis of these communications may have been able to specify the reason for these differences but this could not be accomplished under the time constraints of this study. These results are likely due to the procedural differences in SS:L2. In both CO and CO:CDTl, controllers made ground $\rightarrow$ air and land line PTTs to resolve all aircraft conflicts. In SS:L1, controllers cancelled free flight for many aircraft and made ground $\rightarrow$ air and land line PTTs to resolve the conflicts. In SS:L2, however, controllers were not allowed to cancel free flight and no related instructions (via ground $\rightarrow$ air or land line PTTs) were made to actively maneuver aircraft. In addition, SS:L2 had no scripted pilot inquiries or intent information relays (and, therefore, no controller responses or acknowledgments). These factors likely contributed to the decrease in SS:L2 PTTs compared to the other conditions. 
Finally, controller ratings of situation awareness were high and not significantly different in the four conditions. Controller comments about changing their monitoring style, keeping more situations in mind, and developing alternative plans during shared-separation operations seem to suggest that controller situation awareness should have declined. However, during the debriefing, controllers stated that despite increasing mental workload, they had sufficient information and were able to maintain their awareness of the traffic situations. This information suggests that it was not because of reduced controller situation awareness that controllers felt safety was compromised in the shared-separation conditions. The reasons for controller concerns about safety during shared-separation operations seem directly related to pilot conflict resolution strategies and maneuvers.

In summary, controllers felt that safety was compromised in the shared-separation conditions simulated in the present study. The results from the ground-side indicated that the factors controllers perceived to reduce safety were unpredictable and barely adequate pilot conflict resolution maneuvers that raised controller workload, increased controller stress, and reduced the time available for controllers to intervene. In general, controllers felt that pilots waited too long to resolve conflicts and maneuvered too closely to conflicting aircraft during the sharedseparation conditions. Controllers discussed their concerns about shared-separation operations with the research team and offered valuable insight into their feelings and reactions to the simulation. This feedback is important to understand the issues that need to be addressed should the FAA consider implementing a shared-separation concept in the future.

\subsection{Air-Side Discussion}

The discussion of the air-side data refer only to pilot participant information. Data concerning simulation pilots (WJHTC and NASA) are not included.

\subsubsection{Operational Issues that Affect Shared-Separation Operations}

\subsubsection{Safety measures}

To determine if the conditions had an impact on pilot participant behavior regarding flight safety, the following measures were analyzed: loss of separation and flight crew ratings of safety. There were no losses of separation between the NASA ARC simulator and other aircraft, defined as less than $5 \mathrm{~nm}$ horizontal or 1000/2000 ft vertical separation (as appropriate), during any of the flight segments. Therefore, separation was maintained regardless of who (flight crew or controller) retained separation authority. Keep in mind that there were only three flight crews who flew 36 flight segments. In previous studies using a similar CDTI-AL, a small percentage of runs did result in a loss of separation when pilots attempted to maintain separation without direct involvement of the controller. This included work in both a full-motion simulator (Lozito et al., 2000) and in part-task simulation (Cashion \& Lozito, 2000).

Pilots also reported that for flight safety, SS:L2 was absolutely preferred over CO and strongly preferred over CO:CDTI. The pilots seemed to feel safety was improved when they had more of a role and/or more control over conflict management. Also not surprising was that pilots reported a preference for the CDTI-AL condition over the condition without the CDTI-AL. Perhaps they felt safety was enhanced when they could see the surrounding traffic even when the 
controller retained authority. In addition, the CDTI-AL might have provided them with context as to why they received a course/altitude deviation or enabled them to verify the controller's avoidance solution. Their preference may also reflect improved traffic awareness.

\subsubsection{Aircraft Shared-Separation Performance}

Another safety measure was the time available for the pilot participants to detect conflicting aircraft. The flight crews detected conflicts early on in the flight segments, well before the onset of CDTI-AL alerts. This is similar to findings from other studies of pilots in a shared-separation environment (Lozito et al., 2000). Flight crews' self-reported data corroborates the timing data as they rated the conflicts easy to identify across all of the CDTI-AL conditions. The pilots also rated the CDTI-AL as being effective for shared-separation. It is important to note that flight crews were flying en route flight segments, generally considered a low workload environment under normal conditions. Pilot participants also reported spending a considerable amount of time visually scanning the CDTI-AL. It should be noted that the CDTI-AL features in this study existed on the Navigation Displays for the captain and first officer, and that these displays contain a great deal of navigation data as well as traffic information.

\subsubsection{Flight Efficiency Measures}

Although fuel burn measurements could not be assessed in this study due to the short flight segments, the pilots compared each of the conditions to each other to determine which conditions were preferred for flight efficiency. When asked which conditions they felt were most efficient for their flight segments, the pilots generally rated the shared-separation conditions as more efficient than $\mathrm{CO}$ and $\mathrm{CO}: \mathrm{CDTI}$. Not surprising, these ratings were strongest when comparing SS:L2, the shared-separation condition in which the controllers could not intervene, to CO and CO:CDTI. The pilots absolutely preferred the condition that provided them with the most maneuver flexibility.

\subsubsection{Flight Crew Communication}

Pilot participants generally reported that the air $\leftrightarrow$ air communications were useful to monitor. Pilots also reported they had an adequate amount of time available for communication. However, they only contacted the intruder aircraft in 7 out of 18 conflict situations during the shared-separation conditions. They also rarely contacted other aircraft for any reason. These results are somewhat surprising, as previous research has found that flight crews in a sharedseparation environment often contacted the intruder aircraft to confirm their intentions or query them regarding maneuvering (Lozito et al., 2000). In those previous studies, controllers were not actively controlling aircraft in any of the flight segments. Perhaps the presence of a controller as a backup to provide resolutions and the frequency congestion generated by communications from the controller and other simulation pilots discouraged the use of the air $\leftrightarrow$ air frequency.

\subsubsection{Flight Crew Information Requirements and Procedures}

\subsubsection{Flight Crew Information Requirements}

Form data revealed that the pilot participants found the CDTI-AL and the associated conflict alert logic quite effective for safe operations. They reported that there was only slight confusion 
regarding who had separation authority during the flight segments. However, it should be noted that a few comments by the pilots during data collection suggested that there may have been some confusion regarding procedures and separation authority. As an example, one pilot asked the other if they were still in free flight during one of the flight segments. Participants also indicated that the amount of information on the CDTI-AL was adequate to identify and resolve conflicts and that the timing of the conflict alert was adequate for strategic separation. The flight crew spent a considerable amount of time monitoring the CDTI-AL with the percentage of time increasing as the pilot responsibility for separation increased. This percentage would probably increase with the presence of weather, which was omitted in this study. The amount of heads-down time may be a subject of concern, particularly if there were abnormal events during the flight. The reduced amount of time pilots spent looking out the window or monitoring other displays will need to be considered in the development of new display technology.

The temporal predictors on the CDTI-AL seemed to aid the pilots considerably because they were selected on and in use most of the time. The map range on the CDTI-AL was set to $160 \mathrm{~nm}$ a majority of the time, followed by the $80 \mathrm{~nm}$ range. These data are consistent with ADS-B range capability, and also with prior research regarding CDTI-AL map range settings in free flight (Lozito, McGann, Mackintosh, \& Cashion, 1997; Lozito et al., 2000). Map range settings would also probably be affected by weather because pilots would be looking for weather cells at extended ranges throughout the flight.

\subsubsection{Flight Crew Workload and Situation Awareness}

\subsubsection{Flight Crew Workload}

Two methods were employed to assess flight crew workload, namely Subjective Workload Ratings and AHP Workload Ratings. Both measures required the participants to self-assign workload ratings. The Subjective Workload Ratings were collected after each flight segment and evaluated only in that condition. The AHP Workload Ratings were gathered after completing all runs and provided a comparative assessment of conditions. Workload, as reported by the pilots, averaged from very low to low.

The highest relative workload was reported in one of the shared-separation conditions (SS:L1). In the conditions in which the flight crews flew using current operational procedures ( $\mathrm{CO}$ and $\mathrm{CO}: \mathrm{CDTI}$ ), the tasks were fairly simple for the crews. The shared-separation environment in which the crews were required to inform the controller of maneuvers required more communication. The workload ratings did reveal, however, that even with these new tasks, the flight crew workload was still perceived as low. Furthermore, the flight crews' AHP ratings indicated a strong preference for SS:L2 over CO for reducing their workload. The rest of the conditions were rated equal in value for reducing workload. Perhaps the pilots felt that when they had more control over their own operations, their workload was reduced. This finding is interesting when their physical and mental workloads should have increased with the activities associated with the highest level of shared-separation operations. Another explanation is they may just prefer to be more involved in maintaining separation and therefore always rated SS:L2 as the most favorable condition. 


\subsubsection{Flight Crew Situation Awareness}

The AHP situation awareness data were slightly more conclusive. For situation awareness, SS:L2 was absolutely preferred over $\mathrm{CO}$ (similarly, workload indicated a strong preference). The results also indicated a nearly strong preference for $\mathrm{SS}: \mathrm{Ll}$ over $\mathrm{CO}$ for maintaining situation awareness. Interestingly CO:CDTI was recorded as having only a weak level of dominance for preference over $\mathrm{CO}$.

The pilot participants apparently believed that there was a relationship between the operations of shared-separation and situation awareness. Although they were provided with the CDTI-AL in CO:CDTI, the flight crews did not think that this condition offered much more situation awareness than not having the information at all. However, when allowed the opportunity to use the CDTI-AL to separate themselves, as in SS:L1 and SS:L2, they seemed to feel that their situation awareness was enhanced. The opportunity to use the CDTI-AL information in the shared-separation conditions, along with the responsibility that those procedures entail, seemed to increase their perception of their situation awareness.

In general, the pilot participants had favorable comments regarding the air-side tool and procedures associated with the shared-separation conditions. There were no observable compromises in safety. Pilots did report higher workload for the shared-separation conditions compared to the $\mathrm{CO}$ and $\mathrm{CO}: \mathrm{CDTI}$ conditions, but the workload ratings never reached above the moderate level. However, the ability to generalize from these data is limited by the small sample size, as well as the relatively ideal operational context of the simulation itself (i.e., no weather or abnormal events).

\subsection{Integrated Discussion}

This section discusses findings about the shared-separation concept from an integrated perspective. Table 39 summarizes interesting results of the integrated data for easy referencing.

\subsubsection{Operational Issues that Affect Shared-Separation Operations}

\subsubsection{Safety Measures}

The pilot and the controller participants appeared to have different views regarding the level of safety for shared-separation operations. Pilots seemed to feel that safety was not compromised using the new tools and procedures, but the controllers appeared to have more concerns with the operational concept. However, it is interesting that the pilots essentially rated CO:CDTI as having the same level of safety as $\mathrm{CO}$. They did not appear to make a distinction in safety ratings between the current operations with or without a CDTI.

In SS:L2, pilot tasks and responsibilities increased significantly (e.g., monitoring CDTI-AL, air $\leftrightarrow$ air communications, detecting and resolving conflicts) beyond their normal activities therefore, interestingly, they still perceived SS:L2 as a safer operation. The plausible explanation is that shared-separation procedures that required pilots to look ahead for conflicts and separate themselves may have increased their situation awareness and "pro-active" involvement in their flight planning. However, controllers felt that $\mathrm{CO}$ and $\mathrm{CO}$ CDTI were the 
Table 39. Summary of Integrated Results

\begin{tabular}{|c|c|c|c|c|c|c|c|c|}
\hline & \multicolumn{4}{|c|}{ Controller Participants: Ground-Side } & \multicolumn{4}{|c|}{ Pilot Participants: Air-Side } \\
\hline & $\mathrm{CO}$ & CO:CDTI & SS:LI & SS:L2 & $\mathbf{C O}$ & CO:CDTI & SS:LI & SS:L2 \\
\hline Overall Workload & & & Higher & & \multicolumn{4}{|c|}{ AHP and post nun rating results are not consistent ${ }^{14}$} \\
\hline Mental Workload & & & Higher & & & & \multicolumn{2}{|c|}{ Higher } \\
\hline Physical Workload & & & & & & & \multicolumn{2}{|c|}{ Higher } \\
\hline $\begin{array}{l}\text { Coordination } \\
\text { workload }\end{array}$ & \multicolumn{4}{|c|}{ No difference } & & & Highest & \\
\hline $\begin{array}{l}\text { Communication } \\
\text { workload }\end{array}$ & \multicolumn{4}{|c|}{ No difference } & \multicolumn{4}{|c|}{ No difference } \\
\hline $\begin{array}{l}\text { Situation } \\
\text { Awareness }\end{array}$ & \multicolumn{4}{|c|}{ No difference } & & & \multicolumn{2}{|c|}{ Higher } \\
\hline Loss of separation & 0 & 0 & 0 & 2 & 0 & $\mathbf{0}$ & $\mathbf{0}$ & 0 \\
\hline $\begin{array}{l}\text { Cancellation of } \\
\text { participant pilot } \\
\text { free flight }\end{array}$ & N/A & N/A & $\begin{array}{l}5 \text { out of } 9 \\
\text { times by } \\
\text { controller }\end{array}$ & N/A & N/A & N/A & $\begin{array}{l}\text { None by } \\
\text { participant } \\
\text { pilot }\end{array}$ & $\begin{array}{l}\text { None by } \\
\text { participant } \\
\text { pilot }\end{array}$ \\
\hline $\begin{array}{l}\text { Average horizontal } \\
\text { separation distance } \\
\text { ( participant pilot } \\
\text { conflicts only) }\end{array}$ & $10.5 \mathrm{~nm}$ & $11.0 \mathrm{~nm}$ & $8.2 \mathrm{~nm}$ & N/A & N/A & N/A & $8.7 \mathrm{~nm}$ & $6.2 \mathrm{~nm}$ \\
\hline $\begin{array}{l}\text { Air } \leftrightarrow \text { air } \\
\text { frequency } \\
\text { monitoring }\end{array}$ & $\mathrm{N} / \mathrm{A}$ & N/A & \multicolumn{2}{|c|}{$\begin{array}{c}100 \% \text { of } \mathrm{D} \text {-sides } \\
\text { monitored, but only } \\
50 \% \text { of } \mathrm{R} \text {-sides } \\
\text { monitored. }\end{array}$} & N/A & N/A & \multicolumn{2}{|c|}{$\begin{array}{l}\text { Did not always } \\
\text { monitor. Monitoring } \\
\text { was moderately useful }\end{array}$} \\
\hline $\begin{array}{l}\text { Level of Safety } \\
\text { ratings }\end{array}$ & \multicolumn{2}{|c|}{ Unchanged } & \multicolumn{2}{|c|}{ Compromised } & & & & $\begin{array}{c}\text { Strong } \\
\text { preference }\end{array}$ \\
\hline $\begin{array}{l}\text { Time available to } \\
\text { complete all } \\
\text { communication } \\
\text { and coordination }\end{array}$ & \multicolumn{4}{|c|}{ All Adequate } & $\begin{array}{l}\text { Captains } \\
\text { indicated } \\
\text { more } \\
\text { than } \\
\text { adequate }\end{array}$ & N/A & Adequate & Adequate \\
\hline $\begin{array}{l}\text { Time available to } \\
\text { assure safe } \\
\text { separation }\end{array}$ & Adequate & $\begin{array}{l}\text { Slightly } \\
\text { more than } \\
\text { adequate }\end{array}$ & \multicolumn{2}{|c|}{$\begin{array}{l}\text { Slightly below } \\
\text { adequate }\end{array}$} & N/A & N/A & Adequate & Adequate \\
\hline
\end{tabular}

${ }^{14}$ AHP results indicate reduced workload under SSL2 as compared with CO where as subjective ratings indicate higher workload under SSL2 and SSL1 as compared with CO and CO:CDTI. 
safer operations and reported that SS:L1 and SS:L2 compromised safety. Like the pilots, the controllers did not make a distinction in their safety ratings between the current operations with the presence or absence of a CDTI. However, there do appear to be some concerns with the presence of the new procedures when coupled with the CDTI technology. The fact that controllers cancelled free flight in SS:L1 and indicated that they would have cancelled in SS:L2 reinforces their concern for safety. Thus, the controllers apparently felt that the safety compromise may reside in the new procedures and not in the new technologies and information provided to the flight crews.

\section{Conflict Resolution Strategies}

There appear to be some interesting differences between the pilot and the controller participants with respect to maneuvering strategies. Both controllers and pilots often used heading as part of their resolution strategy when responsible for separation. When responsible for their own conflict resolution (in SS:L1 and SS:L2), pilots may have found heading changes advantageous because their CDTI-AL and navigation display did not provide as much information in the vertical direction ( $\pm 4100 \mathrm{ft}$ only). This could explain why controllers were more likely to use altitude changes in $\mathrm{CO}$ because pilots did not have CDTI-AL. Perhaps controllers were likely to work with heading changes in CO:CDTI and SS:L1 because they were aware that pilots had CDTI-AL that gave them a traffic view and conflict prediction extended beyond the TCAS range. Another notable observation was that the controllers rarely used speed, whereas pilots frequently used speed changes. Because pilot participants were only responsible for their own flight, they may have had more time for conflict management (versus controllers being responsible for all aircraft in the entire sector). For this reason, they may have attempted maneuvering resolutions, which take more time to enact and monitor (e.g., speed changes) or even more complicated strategies (e.g., speed and heading changes together). This may also explain why pilots seemed to use more multiple sub-maneuvers for conflict resolution than controllers do. Corker et al. (2001) also found more prevalent use of a single maneuver resolution by controllers for sharedseparation conflict management.

Interestingly, controllers tended to resolve conflicts earlier than pilots do. It may be that the controllers were resolving conflicts quickly so they could continue to monitor other aircraft. Pilots resolved conflicts rather late (from the controllers perspective) thereby creating minimum impact on their flight plan and efficiency. It must be noted that no pilot participants violated separation minima, and they tended to maneuver before the airborne alert logic triggered display changes to notify the crew of the conflict. This is consistent with previous findings from similar research in the area of free flight (Cashion et al., 1997). Another explanation of these differences may possibly be due to controller roles and responsibilities for the entire sector versus pilot responsibility for only their individual aircraft. As stated previously, pilots may have also had a bit more time to manage their own traffic conflict, particularly in an en route flight segment in which there are no abnormal circumstances. The apparent preference for controllers to solve conflicts early was also reflected in their lower safety ratings during SS:L1 and SS:L2.

Additionally, controllers tended to cancel free flight if the pilots didn't initiate a maneuver and/or didn't inform controllers about their maneuver prior to a point where the controller would have cancelled free flight. Furthermore, when controllers cancelled free flight, they tended to only change the magnitude of the resolution (e.g., increased rate of turn) that was being executed by 
pilots. This may be because the participant pilots' strategies were perceived as correct by the controllers, but the magnitude was simply not enough.

The data indicating MSDs for conflicts involving the pilot participants were also explored. These data revealed that controllers appeared to prefer greater separation distances (than pilots) while actively controlling traffic. In the conditions in which the controllers had full separation authority, $C O$ and CO:CDTI, the MSDs on average were $10.5 \mathrm{~nm}$ and $11 \mathrm{~nm}$ respectively. The instances when the pilots had separation responsibility for themselves, in SS:L1 (unless free flight was cancelled) and always in SS:L2, the average MSDs were $8.7 \mathrm{~nm}$ and $6.2 \mathrm{~nm}$. Controllers seem to be more comfortable with a larger buffer, perhaps again indicating the need to manage their tasks to allow time for the other jobs they must perform. It should be recognized that there is much variance in these data, so it appears that across the relatively small sample size for the pilot and controller participants, there are some differences that may be attributable to individual styles or strategies for controlling and flying aircraft. The observed pilot participant strategies may have also been influenced by their primary domain experience (all oceanic pilots were used) and the fact that pilots are not trained as are controllers, to manage and resolve conflicts.

\subsubsection{Air $\leftrightarrow$ Ground Communication}

The air $\leftrightarrow$ ground communications data may reveal more distinctions between the conditions. The shared-separation condition in which the controllers were able to cancel free flight (SS:L1) seemed to have a pattern indicating a general increase in communications between the controllers and the flight crew participants. As noted, this is partly an artifact of the requirement for the flight crews to inform the controller before executing an avoidance maneuver. The pilots did typically inform the controllers of their intent. Although notification was not always prior to maneuvering, it was usually within 1 minute of the beginning of the maneuver. By contrast, in the shared-separation condition where intent notification was voluntary and controllers couldn't cancel free flight (SSL2), the pilot participants rarely notified the controller of their intended actions. This was likely why there was less total duration of air $\leftrightarrow$ ground communications in SS:L2. The lack of intent relay by the pilot participants was an interesting result because the findings of Corker et al. (2001) indicate the perceived importance of direct relay of intent to controllers in a free flight environment.

Pilot participant ratings of the air $\leftrightarrow$ ground communications revealed that although the duration of communications were different for some conditions, they still had adequate time for air $\leftrightarrow$ ground communications in all conditions.

Missed communications for the four conditions did not appear to indicate any clear finding since the percentage of missed communications relative to total communications was $5 \%$ or less. These percentages are typical of the number of missed communications in the current en route operational environment (Cardosi, 1993). Although SS:L1 has the highest percentage at 5\%, the missed communications in that condition were primarily from a single controller and flight crew pair. 


\subsubsection{Information Requirements and Procedures}

\subsubsection{Cancellation of Free Flight Operations}

By study design, pilots could cancel free flight in SS:L1 and SS:L2 conditions, and controllers could cancel free flight in SS:L1 only. Each condition had a total of 48 conflicts (3 runs of 16). Of the 48 conflicts, 39 involved WJHTC simulation pilots and 9 involved the participant pilots and NASA simulation pilot.

Pilot participants did not cancel free flight during SS:L1 or SS:L2 (nor were there any scripted cancellations by simulation pilots). Other investigations have found similar findings related to the reluctance of flight crews in shared-separation to return separation authority to the controller (Lozito et al., 2000). The pilots seemed to feel that with the new technologies, they were able to maneuver safely to resolve conflicts.

Controllers cancelled free flight in 17 of the 48 conflicts (35\%) in SS:L1. Of the 9 SS:Ll conflicts involving participant pilots, controllers cancelled free flight 5 times (56\%). Of the 39 SS:L1 planned conflicts involving aircraft flown by WHJTC simulation pilots, controllers cancelled free flight 12 times (31\%). Thus, it is clear that the controllers were in some way uncomfortable with the resolution strategy being used by the flight crew participants and the scripted resolutions of the simulation pilots. These data are not too surprising given the novelty of the free flight concept. Previous research has also found high incidences of controller cancellation in free flight scenarios (Corker et al., 2001).

A review of the circumstances of the participant pilot free flight cancellations (5 out of 9 times) indicated that the controllers seemed to cancel free flight when they did not have information of at least one of the following: pilot intent, air $\leftrightarrow$ air communications, and/or pilots first maneuver. It seems likely that a general lack of these types of information to the controller contributed to the discomfort of the controller in shared-separation operations.

\subsubsection{Shared-Separation Procedural Considerations}

Both controllers and pilot participants generally rated the time available for separation tasks as adequate or close to adequate. Based on ratings, the controllers seemed to have slightly more time available to assure aircraft separation in CO:CDTI, although this trend was not very strong. Perhaps the information provided to the flight crew through the CDTI-AL gave the controller more of a perceived time buffer for conflict resolution. Their direct control over the conflict resolution is still intact, but the flight crew is now an extra set of individuals that can closely monitor the traffic and activities around conflicts. The slightly lower ratings for SS:LL and SS:L2 are not surprising because the controllers indicated their safety concerns for these two conditions.

The pilot participants did not rate their time to resolve conflicts as different between the two shared-separation conditions (SS:L1 and SS:L2). In SS:L1, controllers cancelled free flight in five of the nine flight segments. Evidently, the possibility and actual experience of controller cancellation did not impact their sense of conflict resolution timing. 
The controllers and pilots appeared to find that the time allowed in the four conditions for coordination and communication was adequate. This suggests the shared-separation experienced did not adversely affect the time needed for coordination and communications. However, an interesting result was the captains' ratings for $\mathrm{CO}$ as having close to more than adequate time for coordination and communications. The flight crews did not have access to CDTI-AL or air $\leftrightarrow$ air communications in $\mathrm{CO}$. They were not directly responsible for separation and, therefore, they were not engaged in conflict detection and resolution. Perhaps considering their ultimate responsibilities, including fuel consumption, the captains felt that the controllers acted on the conflicts earlier than what was necessary for this condition given the few tasks required by the flight crew. Although many pilots stated in the debriefing that solving conflicts earlier was better, there was some feeling that resolutions may be premature because the situation may remedy itself by a change in flight path on the part of one or both of the conflicting aircraft.

\subsubsection{Additional Information Requirement Considerations}

Controller and pilot subjective comments indicated that URET and CDTI-AL supported their activities rather well. However, in some cases, controllers stated that the URET alerts were too soon or too late. Controller comments also strongly indicated that they would like to have pilot intent information earlier. This need was reinforced by the fact the controllers tended to resolve conflicts earlier than the pilots. If earlier intent information was available to controllers, it is possible that they would not have cancelled free flight as often. At present, CDTI-AL provides alerts about 7 minutes prior to potential loss of separation, whereas URET provides conflict alerts about 13-17 minutes prior to potential loss of separation. Perhaps having similar conflict alert look-ahead times would help pilots formulate and provide their intentions earlier for the controller's needs. (However, the earlier the CDTI-AL alert, the less certain the conflict.) A procedural solution may be to require pilots to provide intentions to controllers at a specified time ahead of the conflict to help alleviate controller anxiety about the lack of timely intentions. On the other hand, perhaps more complicated traffic situations such as the presence of multiple potential conflicts for the same aircraft that would require pilots to execute the maneuvers earlier to avoid complex situations will force the issue,. One of the controllers also suggested larger separation standard for pilots (i.e., $10 \mathrm{~nm}$ ) so that if free flight was cancelled, the controller will have "buffer" separation/time to get the conflict resolved.

Both the controllers and pilot participants indicated that the air $\leftrightarrow$ air frequency was useful in SS:L1 and SS:L2. However, it was distracting at times for R-side controllers and therefore not all R-side controllers used it. Perhaps future technologies, such as datalink, would funnel communications to the appropriate sector, and the air $\leftrightarrow$ air communications would not be as distracting for controllers.

\subsubsection{Workload and Situation Awareness}

\subsubsection{Workload}

In general, based on subjective ratings and form and debriefing comments, controllers indicated that SS:L1 was the most workload intensive and difficult condition. The reasons included increased monitoring tasks, additional tasks to ensure that pilots were resolving conflicts in a safe and timely manner, and planning multiple contingency resolutions for conflicts during 
SS:L1. Controllers tended to prefer CO and CO:CDTI over shared-separation based on their perception of safety and workload. In general, all workload ratings were low, possibly indicating that the shared-separation tasks in this study may not have presented a significant challenge to their overall workload in these lower complexity scenarios. In addition, there was no mix of free flight equipped and non-free flight equipped aircraft in the scenarios. Previous research (Corker et al., 2001; Hilburn et al., 1998) has revealed that high traffic density and mixed equipage does appear to negatively impact controller workload.

Although always rated as relatively low's , the pilot participants appeared to perceive their workload as lower in the CO and CO:CDTI conditions compared to the shared-separation condition with controller notification of intent and possible controller free flight intervention (SS:L1). Interestingly, similar to the controllers, the pilots also indicated that this condition was more workload intensive than the others. This may be because the pilots were required to perform conflict detection and resolution activities, traditionally controller activities, in addition to their normal duties. They were also required to provide intent information under SS:LL. However, the pilots tended to prefer SS:L2 for safety, situation awareness, and workload reasons possibly because it offered them complete autonomy.

\subsubsection{Situation Awareness}

In general, the controllers rated their situation awareness for the four conditions as high, whereas the pilots appeared to believe that the shared-separation conditions provided more situation awareness than the $\mathrm{CO}$ and $\mathrm{CO}: \mathrm{CDTI}$ conditions.

Pilot participants were provided with CDTI-AL in CO:CDTI, SS:L1, and SS:L2. In addition, they were provided an air $\leftrightarrow$ air frequency for communications and new procedures for separation authority in SS:L1 and SS:L2. This combination of new technologies and responsibilities may explain why the pilot situation awareness was higher in SS:L1 and SS:L2. However, although CO:CDTI offered more traffic information through the CDTI-AL, their average situation awareness was reported as the same for CO (without the CDTI-AL).

Controllers were provided with URET information for all four conditions. In addition, for SS:L1 and SS:L2, they received monitoring capabilities of the air $\leftrightarrow$ air frequency and new procedures including shared-separation responsibility. Though all ratings for situation awareness were high, it is somewhat surprising that there was not a noticeable reduction in controller situation awareness for SS:L2. In SS:L2, pilots maneuvered without controller instruction. Informing the controllers about their maneuvers was voluntary, and the pilots (both simulation and participant pilots) rarely provided this intent. Therefore, one would expect overall controller situation awareness in SS:L2 to be reduced. Earlier studies have demonstrated the importance of providing intent to controllers during shared-separation tasks (Corker et al., 2001; Hilburn, Bakker, Pekela,\& Parasuraman, 1997). In addition, one would have expected lower situation awareness in SS:L2 because the procedures seemed to result in the controllers being less directly

\footnotetext{
is One reason for the lower ratings across all conditions may be that the en route flight phase is typically considered low workload for pilots, particularly in conditions such as those simulated where there was no adverse weather and/or emergency events.
} 
engaged in conflict management tasks (especially because no pilots actually cancelled free flight, and separation tasks were never shifted back to the controllers).

\section{CONCLUSIONS}

This study was an initial attempt to examine the concept of shared-separation responsibility using high fidelity simulators for both ATC and flight deck operations. ZME airspace was simulated, and pilots and controllers served as participants. Different levels of shared-separation were examined, with each run containing several conflicting aircraft. Pilots and controllers were provided with display and conflict detection tools to assist in these tasks. Subjective and objective data were analyzed to explore operational issues, provide recommendations for information requirements and procedures, and assess controller and pilot workload and situation awareness.

The key operational issues identified were the differences between controller and pilot perception of safety and conflict resolution strategies. There were no losses of separation while controllers or pilot participants were responsible for separation ${ }^{16}$. However, controllers felt that safety was compromised in shared-separation conditions when compared to the current operational conditions. The number of cancelled free flight operations in SS:L1 and the controllers' indications that they would have cancelled free flight in several situations in SS:L2 were evidence of their concern about safety issues. In contrast, the pilot participants rated both shared-separation conditions as being relatively safer than current operations. The pilots indicated an overall preference for the shared-separation conditions, particularly when they had the most flexibility and separation authority (SS:L2). Apparently, the perceived flexibility that shared separation provided for the pilots seemed to result in safety concerns and discomfort for the controllers. There were also some interesting differences between the pilots and controllers with respect to conflict resolution strategies. These included maneuvering styles, timing of action, and separation distances. For conflict resolution, controllers preferred the use of heading and altitude, whereas pilots preferred heading and speed maneuvers. Controllers tended to resolve conflicts earlier and seemed to prefer greater separation distances than pilots.

Both pilot and controller participants generally found their tools for their relevant tasks to be useful and sufficient. However, the notable issues related to information requirements and procedures included the availability of intent knowledge, the cancellation of free flight, and the harmonization of conflict detection tools. The lack of timely pilot intent knowledge (as perceived by controllers) seemed to result in free flight cancellations by controllers. They also stated that this added to their workload and discomfort with shared-separation operations. If earlier intent information was provided to controllers, it is possible that they would not have cancelled free flight as often as they did. Perhaps having harmonized conflict alert look-ahead times would help pilots formulate and provide their intentions earlier for controller needs. In

\footnotetext{
${ }^{16}$ There were two losses of separation in SS:L2 runs (controllers could not cancel free flight) due to late maneuvering (script execution error) by the WJHTC simulation pilots.
} 
addition, both controllers and pilots found the capability of air $\leftrightarrow$ air communications useful; however, a more sophisticated technology would need to be developed.

Controller and pilot workload was generally low for all conditions, though there were some interesting observations. Both controllers and pilot participants indicated that $\mathrm{SS}: \mathrm{Ll}$ was the most workload intensive scenario. Controllers also stated that it was difficult for them and suggested that their higher workload was due to increased monitoring, continuous assessment of pilot solutions, and the need to plan contingency resolutions. Though pilots stated they preferred shared-separations operations, they may have rated workload higher in SS:Ll because, in addition to their normal duties, they had to provide intent information and perform conflict detection and resolution activities. Controller situation awareness was consistently high for all conditions, but pilots reported greater situation awareness during shared-separation conditions compared to current operations. Their higher situation awareness may be explained by the combination of new technologies and experienced responsibilities.

In this limited investigation of the shared-separation concept, pilot participants tended to prefer shared-separation conditions, whereas the controller participants tended to prefer current operational conditions. These data were based on a small sample size and simplified scenarios and events. For example, this simulation did not consider weather, climbing and descending aircraft, or other factors that add to the complexity of air traffic operations. Future research should investigate the shared-separation concepts with multiple aircraft conflicts, simultaneous conflicts, and aircraft in successive conflicts. Finally, mixed equipage operations in a sharedseparation context is another characteristic that is likely to have a strong impact on these operations and therefore needs to be investigated. Although it is premature to identify the best possible shared-separation level, the results of this study demonstrate the need to conduct further research in this area. 


\section{ACRONYMS}

$\begin{array}{ll}\text { ACID } & \text { Aircraft Identifier } \\ \text { ADO } & \text { Automatic Datalink Operator } \\ \text { ADS-B } & \text { Automatic Dependant Surveillance-Broadcast } \\ \text { AGIE } & \text { Air-Ground Integration Experiment } \\ \text { AHP } & \text { Analytical Hierarchy Process } \\ \text { ANOVA } & \text { Analysis of Variance } \\ \text { ARC } & \text { Ames Research Center } \\ \text { ARTCC } & \text { Air Route Traffic Control Center } \\ \text { ATC } & \text { Air Traffic Control } \\ \text { CDTI } & \text { Cockpit Display of Traffic Information } \\ \text { CDTI-AL } & \text { Cockpit Display of Traffic Information with Alerting Logic } \\ \text { CPC } & \text { Certified Professional Controller } \\ \text { CO } & \text { Current Operations } \\ \text { CO:CDTI } & \text { Current Operations with Cockpit Display of Traffic Information with } \\ & \text { Alerting Logic } \\ \text { CVSRF } & \text { Crew Vehicle Systems Research Facility } \\ \text { DSR } & \text { Display System Replacement } \\ \text { EO } & \text { Expert Observers } \\ \text { FAA } & \text { Federal Aviation Administration } \\ \text { FAR } & \text { Federal Aviation Regulations } \\ \text { FL } & \text { Flight Level } \\ \text { FMC } & \text { Flight Management Computer } \\ \text { FMS } & \text { Flight Management System } \\ \text { IF } & \text { Integration and Interoperability Facility } \\ \text { IP } & \text { Internet Protocol } \\ \text { LIT } & \text { Little Rock } \\ \text { M } & \text { Mean } \\ \text { MCDU } & \text { Multi-Function Control Display Unit } \\ \text { MSD } & \text { Minimum Separation Distance } \\ \text { NAS } & \text { National Airspace System } \\ \text { NASA } & \text { National Aeronautics and Space Administration } \\ \text { OS } & \text { Operations Supervisor } \\ \text { PAS } & \text { Pseudo Aircraft Systems } \\ \text { PTT } & \text { Push-to-Talk Transmissions } \\ \text { PVD } & \text { Plan View Display } \\ \text { RA } & \text { Resolution Advisory } \\ \text { SAR } & \text { System Analysis and Recording } \\ \text { SD } & \text { Standard Deviation } \\ \text { SEM } & \text { Standard Error of the Mean } \\ \text { SS:L1 } & \text { Shared-Separation Level I } \\ \text { SS:L2 } & \text { Shared-Separation Level 2 } \\ \text { SUA } & \text { Special Use Airspace } \\ \text { TA } & \text { Traffic Advisory } \\ \text { TCAS } & \text { Traffic Alert and Collision } \\ & \end{array}$




$\begin{array}{ll}\text { TGF } & \text { Target Generation Facility } \\ \text { URET } & \text { User Request Evaluation Tool } \\ \text { VNTSC } & \text { Volpe National Transportation Systems Center } \\ \text { VOR } & \text { Very High Frequency Omni Directional Radio Range } \\ \text { VORTAC } & \text { VOR Tactical Air Navigational Aid } \\ \text { WAK } & \text { Workload Assessment Keypad } \\ \text { WJHTC } & \text { William J. Hughes Technical Center } \\ \text { ZME } & \text { Memphis ARTCC }\end{array}$




\section{REFERENCES}

Cardosi, K. (1993). An analysis of en route controller-pilot voice communications (DOT-FAARD-93/11). Cambridge, MA: Volpe National Transportation Systems Center.

Cashion, P. \& Lozito, S. (2000, September). How short and long-term intent information affects pilot performance in a free flight environment. Paper presented at the $\mathrm{HCI}$ Aero Conference, Toulouse, France.

Corker, K., Fleming, K., \& Lane, J. (2001). Air-ground integration dynamics in the exchange of information for control. In L. Bianco, P. Dellílmo, \& A. Odoni (Eds.), Transportation Analysis: New Concepts and Methods in Air Traffic Management (pp. 125-142). Berlin: Springer Verlag.

Endsley, M. R. (1997). Situation awareness, automation and free flight. Cambridge, MA: Massachusetts Institute of Technology.

Endsley, M. R., Mogford, R. H., Allendoefer, K. R., Snyder, M. D., \& Stein, E. S. (1997). Effect of free flight conditions on controller performance, workload, and situation awareness (DOT/FAA/CT-TN97/12). Atlantic City International Airport, NJ: William J. Hughes Technical Center.

Endsley, M. R., Sollenberger, R. L., Nakata, A., \& Stein, E. S. (2000). Situation awareness in air traffic control: Enhanced displays for advanced operations (DOT/FAA/CT. TN00/01). Atlantic City International Airport, NJ: William J. Hughes Technical Center.

Federal Aviation Administration (2000). Air traffic control (DOT/FAA/Order 7110.65M). Washington, DC: Federal Aviation Administration.

Federal Aviation Administration (1998). ATS concept of operations for the national airspace system in 2005. Washington, DC: Federal Aviation Administration.

Federal Aviation Regulations (1997). Federal aviation regulations: Pt. 91, general operating and flight rules (DOT/FAA/O/N97-237). Washington, DC: Federal Aviation Administration.

Hilburn, B. G., Bakker, M. W. P., \& Pekela, W. D. (1998). Free flight and the air traffic controller: an exploratory analysis of human factors issues (NLR-TP-98237). Amsterdam: The Netherlands.

Hilburn, B. G., Bakker, M. W., Pekela, W. D., \& Parasuraman, R. (1997). The effect of free flight on air traffic controller mental workload, monitoring, and system performance. Proceedings of the Free Flight Tenth European Aerospace Conference. Amsterdam: The Netherlands. 
Johnson, W., Battiste, V., \& Bochow, S. H. (1999). A cockpit display designed to enable limited flight deck separation responsibility. Proceedings of the 1999 World Aviation Conference, San Francisco, CA.

Kerns, K. (1999). Effects of a strategic conflict probe capability and unstructured traffic conditions on ATC performance and flight efficiency (MTR 98W0000121). McLean, VA: MITRE Center for Advanced Aviation System Development.

Lozito, S., McGann, A., Cashion, P., Dunbar, M., Mackintosh, M., Dulchinos, V., \& Jordan, K. (2000). Free flight simulation: An initial examination of air-ground integration issues (NASA Tech Memorandum - 2000-209605). Moffett Field, CA: NASA-Ames Research Center.

Lozito, S., McGann, A., Mackintosh, M., \& Cashion, P. (1997, June). Free flight and selfseparation from the flight deck perspective. Paper presented at the lst USA/Europe Air Traffic Management Research and Development Seminar, Saclay, France.

Mackintosh, M, Dunbar, M., Lozito, S., Cashion, P., McGann, A., Dulchinos, V., Ruigrok, R., Hoekstra, J., \& Van Gent, R. (1998, December). Self-separation from the air and ground perspective. Paper presented at the Second USAVEurope Air Traffic Management Research and Development Seminar, Orlando, FL.

National Aeronautics \& Space Administration (1999). Concept definition for distributed air/ground traffic management, v1.0. Moffett Field, CA: NASA.

Pekela, W. D. \& Hilburn, B. (1998, September). Air traffic controller strategies in resolving free flight traffic conflicts: The effect of enhanced controller displays for situation awareness. Proceedings of the 1998 World Aviation Conference, Anaheim, CA.

RTCA (1995). Final report of RTCA task force 3: Free flight implementation. Washington, DC: RTCA.

RTCA (1992). Minimum operational performance standards for airborne automatic dependent surveillance (ADS) equipment (RTCADO-212). Washington, DC: RTCA.

Smith, P. J., Billings, C., McCoy, E. C., \& Orasanu, J. (1999). Alternative architectures for distributed cooperative problem solving in the national airspace system. Ohio: Ohio State University, Ohio University, and NASA Ames Research Center.

Sullivan, B. T., \& Soukup, P. A. (1996, July). The NASA 747-400 flight simulator: National resource for aviation safety research (AIAA-96-3517). Paper presented at the American Institute of Aeronautics and Astronautics, San Diego, CA.

Yang, L., \& Kuchar, J. (1997). Prototype conflict alerting logic for free flight (AIAA 97-0220). Reston, VA: American Institute of Aeronautics and Astronautics. 


\section{GLOSSARY OF TERMS}

\begin{tabular}{|c|c|}
\hline URET & $\begin{array}{l}\text { User Request Evaluation Tool. The look-ahead time for conflict } \\
\text { detection using flight plan on the URET is approximately } 20 \text { minutes. }\end{array}$ \\
\hline CDTI-AL & $\begin{array}{l}\text { Cockpit Display of Traffic Information with Alerting Logic. The } \\
\text { CDTI-AL typically alerts the flight crews approximately 6-7 minutes } \\
\text { prior to the closet point of approach between aircraft. }\end{array}$ \\
\hline Workload & $\begin{array}{l}\text { Workload is defined as combined cognitive and physical demands } \\
\text { experienced by an operator. The workload experienced by an operator } \\
\text { depends on the task, skill, knowledge, experience, abilities, and } \\
\text { training. Generally, workload is considered as an operator's response } \\
\text { to taskload. }\end{array}$ \\
\hline $\begin{array}{l}\text { Situation } \\
\text { Awareness }\end{array}$ & $\begin{array}{l}\text { Situation awareness is defined as an operator's ability to integrate } \\
\text { information related to state of a task, operation, equipment, and } \\
\text { environment; make necessary predictions; and take the necessary } \\
\text { decisions and suitable actions. Several other definitions of situation } \\
\text { awareness exist. }\end{array}$ \\
\hline Communication & $\begin{array}{l}\text { A series of two or more transmissions between a member of the flight } \\
\text { crew and a controller on a single topic; an exchange of a message and } \\
\text { a response; the duration of a transaction is measured from the onset of } \\
\text { speech for the initial message to the end of the response from the other } \\
\text { party. }\end{array}$ \\
\hline Transmission & $\begin{array}{l}\text { A verbal message from the controller to the pilot or vice versa; the } \\
\text { duration of which is measured from the onset of speech to the end of } \\
\text { the message (offset of speech). }\end{array}$ \\
\hline $\mathrm{CO}$ & $\begin{array}{l}\text { This condition emulates today's ATC environment; that is, the } \\
\text { controller is responsible for separation assurance of all aircraft. } \\
\text { URET is operational. }\end{array}$ \\
\hline CO:CDTI & $\begin{array}{l}\text { This condition emulates today's ATC environment. URET is } \\
\text { operational. In this condition, however, the flight crews have } \\
\text { CDTI-AL available to them. }\end{array}$ \\
\hline SS:L1 & $\begin{array}{l}\text { This condition emulates a subset of the RTCA definition of the free } \\
\text { flight environment. URET and CDTI-AL are operational. Controllers } \\
\text { have specific procedures for coordinating URET alerts. Flight crews } \\
\text { are free to initiate any maneuver (i.e., change heading, altitude, or } \\
\text { speed), provided they first inform ATC of their intentions. Controllers } \\
\text { and flight crews both could cancel free flight. }\end{array}$ \\
\hline SS:L2 & $\begin{array}{l}\text { This condition incorporates all the conditions of SS:L1 with some } \\
\text { modifications. Flight crews are free to maneuver, however they are } \\
\text { not required to inform ATC of their intentions. Flight crews may } \\
\text { cancel free flight, but controllers can not. }\end{array}$ \\
\hline
\end{tabular}




\section{Appendix A}

\section{Controller Daily Schedule}




\section{$\checkmark$}

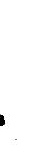


Daily Schedule for Controllers

\begin{tabular}{|c|c|c|c|c|c|}
\hline Time (EST) & Day 1 & Day 2 & Day 3 & Day 4 & Day 5 \\
\hline $\begin{array}{l}12: 00 \mathrm{pm} \\
: 15\end{array}$ & \multirow{20}{*}{$\begin{array}{l}\text { Participant } \\
\text { Travel }\end{array}$} & \multirow{2}{*}{$\begin{array}{l}\text { Controller } \\
\text { Briefing }\end{array}$} & \multirow{5}{*}{ Training } & $\begin{array}{l}\text { Review } \\
\text { Review }\end{array}$ & \multirow{20}{*}{$\begin{array}{l}\text { Participant } \\
\text { Travel }\end{array}$} \\
\hline $\begin{array}{l}: 30 \\
: 45 \\
\end{array}$ & & & & \multirow[b]{2}{*}{ Run 3} & \\
\hline $\begin{array}{c}01: 00 \mathrm{pm} \\
: 15 \\
: 30 \\
: 45 \\
\end{array}$ & & \multirow{5}{*}{$\begin{array}{c}\text { ATC } \\
\text { Laboratory } \\
\text { Familiarization }\end{array}$} & & & \\
\hline $\begin{array}{l}\text { 02:00pm } \\
: 15\end{array}$ & & & & $\begin{array}{c}\text { Forms \& } \\
\text { Discussion }\end{array}$ & \\
\hline $\begin{array}{l}: 30 \\
: 45\end{array}$ & & & & \multirow{3}{*}{$\begin{array}{c}\text { MEAL } \\
\text { BREAK }\end{array}$} & \\
\hline $03: 00 \mathrm{pm}$ & & & \multirow{2}{*}{$\begin{array}{c}\text { MEAL } \\
B R E A K\end{array}$} & & \\
\hline$: 15$ & & & & & \\
\hline $\begin{array}{l}: 30 \\
: 45 \\
\end{array}$ & & MEAL & & & \\
\hline $\begin{array}{c}\text { 04:00pm } \\
: 15\end{array}$ & & $B R E A K$ & Run 1 & Run 4 & \\
\hline $\begin{array}{l}: 30 \\
: 45 \\
\end{array}$ & & Training Run I & & & \\
\hline $05: 00 \mathrm{pm}$ & & & & Forms \& & \\
\hline$: 15$ & & Discussion & Forms \& & Discussion & \\
\hline$: 30$ & & BREAK & Discussion & $B R E A K$ & \\
\hline$: 45$ & & \multirow{2}{*}{ Training Run 2} & $B R E A K$ & \multirow{3}{*}{ Debriefing } & \\
\hline 06:00pm & & & \multirow{4}{*}{ Run 2} & & \\
\hline $\begin{array}{l}: 15 \\
: 30\end{array}$ & & Discussion & & & \\
\hline$: 45$ & & $B R E A K$ & & Buffer & \\
\hline $\begin{array}{c}\text { 07:00pm } \\
: 15\end{array}$ & & \multirow[t]{2}{*}{ Training Run 3} & & $\begin{array}{l}\text { Buffer } \\
\text { Buffer }\end{array}$ & \\
\hline$: 30$ & & & \multirow{2}{*}{$\begin{array}{c}\text { Forms \& } \\
\text { Discussion }\end{array}$} & Buffer & \\
\hline$: 45$ & & Discussion & & Buffer & \\
\hline
\end{tabular}




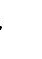

$$
=
$$




\section{Appendix B}

Controller Briefing 
.

$$
=
$$

$$
\Delta
$$




\section{Controller Briefing}

\section{$\underline{\text { Air-Ground Integration Experiment (AGIE) }}$}

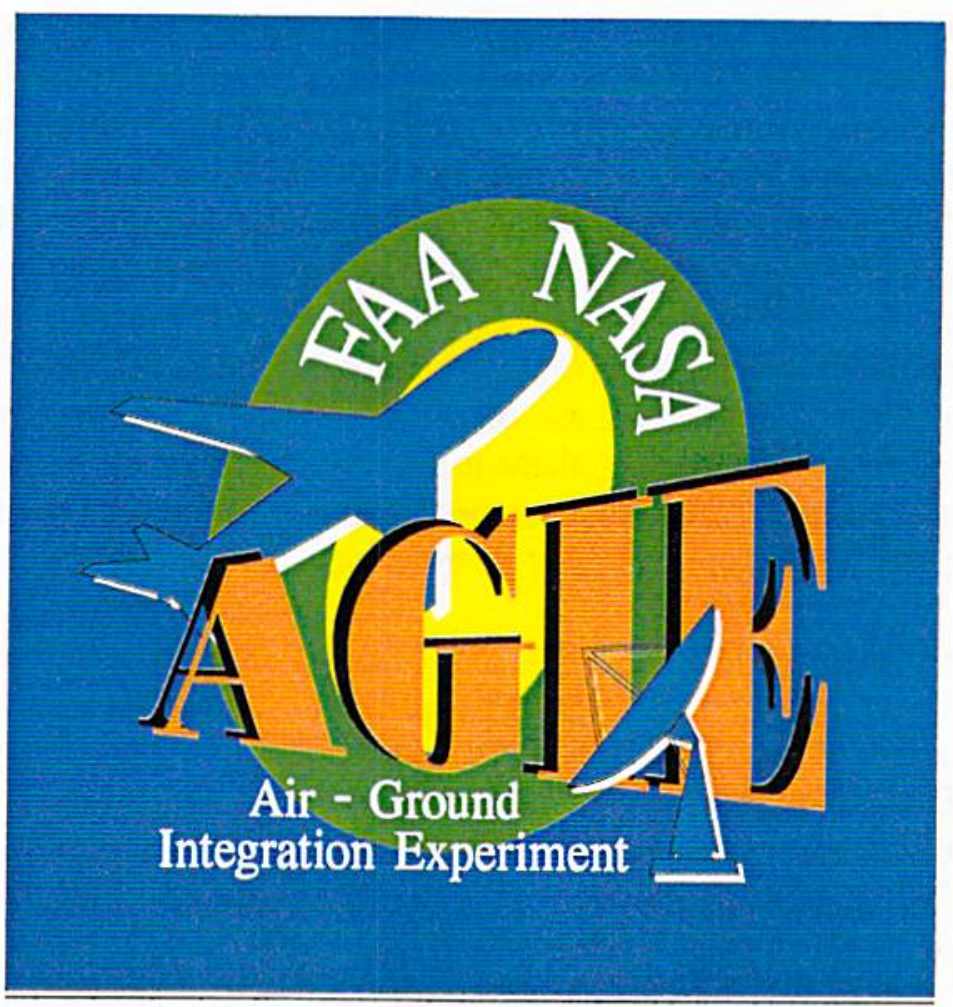

FAA William J. Hughes Technical Center Points of Contact

Karen DiMeo, ACT-540

Dr. Randy Sollenberger, ACT-530 


\section{Controller Briefing \\ Air-Ground Integration Experiment}

\section{Introduction}

Over the last decade, the recognized need for more efficient Air Traffic Control (ATC) has produced several new automation systems. The Federal Aviation Administration (FAA) has implemented programs such as the Enhanced Traffic Management System (ETMS) and the Center TRACON Automation System (CTAS) to assist air traffic controllers and managers with their duties. For the cockpit, automated Flight Management Systems have been developed to help flight crews plan and execute fuelefficient routings to destination airports. Systems such as these are intended to provide means for more efficient use of the National Airspace System (NAS). However, the fact remains that the tools in the cockpit and on the ground are not integrated thereby limiting the maximum benefit obtained from these technologies. In the spirit of progress, the FAA continues to address such problems by testing and implementing new technologies and procedures, and by investigating future concepts.

'Free Flight' as described by the RTCA Task Force 3, sets forth a concept of future air traffic management that requires a tightly integrated system to meet the freer and more collaborative nature of air traffic management in the future. While aircraft-to-aircraft separation will remain the responsibility of service providers, and in most cases, will remain solely their responsibility, today's practice of visual separation by pilots in terminal areas is expanded to allow all-weather pilot separation when feasible. The increased use of shared-separation responsibility will be possible through the use of traffic displays on the flight deck, as well as rules, procedures, and training programs that modify the roles and responsibilities of users and service providers. Human factors analyses and human-in-the-loop simulations will help determine the appropriate allocation of tasks between service providers, users, and automation systems.

In response, the National Aeronautics and Space Administration (NASA) Ames Research Center (ARC) has developed a Cockpit Display of Traffic Information (CDTI) prototype. The CDTI includes embedded conflict alerting logic (AL) that predicts the probability of an encounter with another aircraft. The CDTI-AL relies on Automated Dependant Surveillance (ADS) technology to supply the position and trajectory information of all air traffic in the vicinity. This prototype 'decision support tool' is intended to enhance flight crews' situation awareness and provide them more autonomy in the NAS. In addition, the MITRE Corporation has developed a ground-based conflict probe and trial-planning tool for use by air traffic controllers. This prototype decision support tool, entitled User Request and Evaluation Tool (URET), is currently being evaluated at the Indianapolis and Memphis Air Route Traffic Control Centers. While there have been studies done on each of these tools individually, there is a need to investigate how they might work together in a shared-separation environment. 
An Experiment Working Group (EWG) has been formed to investigate the effects of shared-separation authority on flight operations when both the air and ground have enhanced traffic and conflict alerting systems. The EWG is represented by FAA Head Quarters, the FAA William J. Hughes Technical Center (WJHTC), NASA ARC, and the Volpe National Transportation Systems Center (VNTSC). The study is co-sponsored by the FAA (AAR-100, ASD-130, ATP-400) and NASA (Advanced Air Transportation Technologies Program).

AGIE is the first high fidelity, real-time, human-in-the-loop simulation in a planned series of studies to investigate shared-separation operations. More studies will be conducted in the future prior to making any conclusive recommendations about sharedseparation responsibility.

\section{Objectives}

This experiment is intended to provide an initial examination of the effect of sharedseparation authority on flight operations when both air and ground have enhanced traffic and conflict alerting systems. There will be strong emphasis on identifying and evaluating the human factors impact. Under the conditions simulated, the specific objectives are:

- Identify operational issues (e.g., communications, procedures, etc.) that affect sharedseparation operations.

- Provide recommendations for the information requirements and procedures necessary to facilitate shared-separation operations.

- Evaluate the effect of shifting separation authority on controller and pilot workload and situation awareness.

\section{Definitions and Terms}

The following provides description of definitions and terms that are essential in this experiment.

\section{- URET-User Request Evaluation Tool.}

The look ahead time for conflict detection using flight plan on the URET is approximately 20 minutes. You will be required to coordinate RED alerts with adjacent sectors. The URET will be operational under all scenarios.

- CDTI-AL-Cockpit Display of Traffic Information with Alert Logic. The CDTI-AL typically alerts the flight crews approximately 6-7 minutes prior to the closet point of approach between aircraft. Therefore, in most cases URET will provide conflict alerts prior to the CDTI-AL. See Appendix A for detailed description.

- $\quad \underline{\mathbf{I}^{2} \mathbf{F}}-\quad$ Integration and Interoperability Facility (Building 27)

- RDHFL- Research Development and Human Factors Laboratory (Building 28) 
- WAK- Workload Assessment Keypad

\section{Scenario Descriptions}

Following training, you will be presented four scenario types in a random order. This section contains a description of each scenario, conflict resolution and coordination information, phraseology conditions, and your role while participating.

\subsection{Training scenarios}

There will be three training scenarios, one each for CO:CDTI, SS:L1, and SS:L2 operations. See below for descriptions of scenario characteristics.

\subsection{Baseline (CO) scenario characteristics}

This control condition will simulate today's ATC environment. URET will be operational. Standard air traffic procedures defined in the Controller Handbook 7110.65, Federal Aviation Regulations (FAR) Part 91, and the Aeronautical Information Manual will apply.

- Today's air traffic control operation where controllers have separation responsibility.

- Pilots do not have access to CDTI-AL in the cockpit.

- Controllers have access to URET.

- Pilots cannot initiate a maneuver without ATC clearance.

\subsection{CO:CDTI scenario characteristics}

This control condition will simulate today's ATC environment with the addition that the flight crews will have access to CDTI-AL. URET will be operational. Standard air traffic procedures defined in the Controller Handbook 7110.65, FAR Part 91, and the Aeronautical Information Manual will apply. Pilots may request alternate routes to maximize fuel efficiency or when potential conflicts are detected with the CDTI-AL, but controllers retain the authority to approve/deny all pilot requests.

- Controllers will take the initiative to resolve conflicts between aircraft.

- Pilots do have access to CDTI-AL in the cockpit.

- Controllers have access to URET.

- Pilots cannot take any action (other than emergency or TCAS RA) without clearance from ATC.

- Pilots can query controllers (e.g. about potential conflicts or traffic) and make requests based on information from their CDTI-AL displays.

\subsection{SS:L1 scenario characteristics}

This control condition will emulate a subset of the RTCA definition of the Free Flight environment. URET and CDTI-AL will be operational. The initial flight plan and altitude will be considered as optimum for the current conditions. Standard air traffic rules defined in the Controller Handbook 7110.65, FAR Part 91 and the Aeronautical 
Information Manual will still apply, with the major exception that flight crews will initially provide their own separation. However, prior to maneuvering, they must first inform ATC of their intended actions. Controllers can cancel Free Flight.

- Shared-separation operation and responsibility.

- Pilots do have access to CDTI-AL in the cockpit.

- Controllers have access to URET.

- Flight crews are free to maneuver in any direction including vertically provided they first inform ATC.

- An air-to-air frequency is available. Controllers can monitor the air-to-air frequency as desired, but it is not required.

- Standard separation rules of 5 miles laterally or $1000 / 2000 \mathrm{ft}$. vertically shall be observed by ATC and flight crews.

- To issue a control instruction to a pilot, controllers must CANCEL their Free Flight operation.

- Controllers may only CANCEL FREE FLIGHT (for one or a pair of aircraft, sectorwide cancellation is NOT allowed) if they have queried at least one of the subject aircraft as to pilot's intentions.

- Aircraft whose free flight has been canceled will remain under ATC control unless/until the controller RESUMES Free Flight.

- Controllers shall update the Host/DSR database when flight plans are changed.

- Controllers shall issue Traffic Alerts using prescribed phraseology to the aircraft involved in a RED URET alert.

- Controllers shall coordinate all RED URET alerts on aircraft not under their control with the controlling sector using the prescribed phraseology.

- Controllers receiving a coordinated Traffic Alert shall forward this to the subject aircraft unless that aircraft has already advised that a resolution is in progress.

- Controllers shall have the prerogative to wait to issue a Traffic Alert until the subject aircraft is under his/her control.

- Controllers shall coordinate any aircraft action that will affect another controller's airspace.

- Flight crews can cancel Free Flight for their aircratt and request ATC to intervene at any time.

\subsection{SS:L2 scenario characteristics}

This control condition incorporates all the conditions of SS:L1 with the following changes:

- Flight crews are not required to inform the controller before initiating any maneuver.

- Controllers are not required to issue Traffic Alerts to aircraft, but may do so.

- Controllers must still coordinate all RED URET alerts on aircraft not under their control with the controlling sectors.

- Controllers may NOT CANCEL FREE FLIGHT; however, conflict detection and resolution measures will be collected. 
- Flight crews can cancel Free Flight for their aircraft and request ATC to intervene at any time.

\subsection{Conflict resolution rules}

During SS:L1 and SS:L2 scenarios, pilots are instructed to use FAR Part 91 right-of-way rules (when possible) while resolving conflicts. The pilot right-of-way rules are as follows:

- The aircraft on the others right has the right-of-the-way.

- The aircraft being overtaken has the right-of-the-way.

- The aircraft that are head-on should each alter course to the right.

- During most conflict situations, the aircraft that does not have right-of-way will initiate the coordination with the aircraft that has right of way.

\subsection{Coordination of URET red alerts}

You will be required to coordinate RED URET alerts with other sectors under all scenario types. In other words, you will coordinate all RED alerts for those aircraft where the conflict is predicted to occur in your sector, but the aircraft is not within your sector and/or control. However you need not coordinate RED alerts with "brown" URET aircraft ID's or those with "UNK" as the sector. For simulation purposes, these are considered "nuisance" RED alerts.

\subsection{Phraseology}

Controllers will use standard phraseology as described in the FAA Order 7110.65 to minimize the possibility of misunderstandings among other controllers and pilots. In addition, the following phraseology will be used in this simulation:

\subsubsection{Simulation Phraseology}

- Cancellation of Free Flight (SS:L1 only).

Controller - "ACID (and $A C I D$ ), Free Flight canceled" and issue the appropriate control action.

- Resumption of Free Flight (SS:L1 only).

Controller - " $A C I D$ (and $A C I D$ ), resume Free Flight."

- Acknowledge Pilot intentions (SS:Ll only).

Pilot - informs controller of an intended maneuver.

Controller - "ACID, roger."

- Aircraft coordination for RED URET alerts (SS:L1 and SS:L2).

Controller - " $A C I D$, traffic alert with $A C I D$ at (altitude) at (time), advise intentions" 
Sector coordination for RED URET alerts (SS:L1 and SS:L2).

Controller Sector 1 - "Traffic alert ACID"

Controller Sector 2 - "Go ahead"

Controller Sector 1 - "ACID with $A C I D$ at altitude at time"

Controllers may continue to use their operating initials at the end of communications as usual.

Pilots will be trained on simulation phraseology for their communications on the air-toair frequency.

Controllers will be given an opportunity to comment on all phraseology at the end of this experiment.

The following table provides a summary of scenario characteristics.

Table 1. Scenario Description Summary

\begin{tabular}{|c|c|c|c|c|}
\hline Charactertatica & $\mathbf{C O}$ & CO:CDTI & SS:L1 & SS:L2 \\
\hline URET available & $\sqrt{ }$ & 4 & $\frac{1}{4}$ & $\sqrt{5}$ \\
\hline Need to coordinate URET red alerts with other sectors & $\sqrt{ }$ & $\checkmark$ & $\checkmark$ & $\mathbf{J}$ \\
\hline Controllers have full separation responsibility & $\sqrt{v}$ & 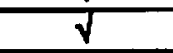 & & \\
\hline $\begin{array}{l}\text { Pilots must request a clearance from controllers prior to flight } \\
\text { plan changes }\end{array}$ & $\checkmark$ & $\sqrt{ }$ & & \\
\hline CDTl-AL available & & $\mathbf{v}$ & $\sqrt{ }$ & $\mathbf{v}$ \\
\hline Air-to-air frequency available & & & $\sqrt{ }$ & $\checkmark$ \\
\hline Pilots use right-of-way rules while resolving potential conflicts & & & $\checkmark$ & v \\
\hline $\begin{array}{l}\text { Pilots and controllers have shared-separation responsibility - } \\
\text { Pilots can cancel free flight }\end{array}$ & & & $\checkmark$ & $\checkmark$ \\
\hline New controller phraseology & & & ง & ป \\
\hline Pilots must inform controllers prior to flight plan changes & & & T & \\
\hline $\begin{array}{l}\text { Controllers acknowledge flight plan changes using the } \\
\text { phraseology "Roger" }\end{array}$ & & & 守 & \\
\hline $\begin{array}{l}\text { Pilots and controllers have shared-separation responsibility - } \\
\text { Controllers can cancel Free Flight }\end{array}$ & & & T & \\
\hline $\begin{array}{l}\text { Pilots and controllers have shared-separation responsibility - } \\
\text { Controllers will report when the would cancel Free Flight, but } \\
\text { can not actually cancel }\end{array}$ & & & & $\sqrt{ }$ \\
\hline $\begin{array}{l}\text { Pilots do not have to inform controller prior to flight plan } \\
\text { changes }\end{array}$ & & & & $\sqrt{ }$ \\
\hline
\end{tabular}

\section{Experiment Procedure}

You will be participating in this study for a period of 3 days (from 12 to 8 p.m.). On the first day, there will be an experiment briefing and training. The experiment briefing will provide an overview of the experiment, objectives, and your role. The training will provide you with laboratory familiarization and an opportunity to control air traffic under the different control conditions of the experiment. On the second day, you will be provided additional training as necessary. Following the training, data collection runs 
will begin. The data collection runs will continue through the third day. At the end of all data collection runs, there will be an unstructured, group debriefing session. The purpose of this debriefing is to provide you an opportunity to share information that is not captured in the forms.

On the first day, you will be given a consent form and a background data collection form. After completing these forms, you will participate in three training runs, each corresponding to CO:CDTI, SS:L1, and SS:L2. This will conclude the first day. At the beginning of the second day, you will be given more opportunity for training. Following the training runs, the data collection runs will begin.

At the beginning of each run, a researcher will inform you of the control condition (e.g., CO:CDTI, SS:L1, SS:L2, and CO). You will be provided with an aid at the control position describing rules of the operation. You will also be given a sector briefing as you join the run. Except for training scenarios, the duration of each scenario is $\mathbf{9 5}$ minutes. The training scenarios are $\mathbf{4 5}$ minutes long.

There will be two Expert Observers in the control room. One observer will be assigned to sector 44 and the other will be assigned to sector 21. During each scenario, the observers will watch sector operations and record interesting and critical events. The observers will be either a current controller or a current supervisor.

Participation in this study is strictly voluntary and the privacy of participants will be protected. No individual names or identities will be recorded or released in any reports. Therefore, you will be assigned a participant code (e.g., $\mathrm{Cl}, \mathrm{C} 2, \mathrm{C}$, and $\mathrm{C} 4$ ) that will remain the same throughout the experiment. Strict adherence to all federal, union, and ethical guidelines will be maintained throughout the study.

You will be assigned a specific $\mathrm{R}$ or D-side position for sector 21 or sector 44 . Your sector and position assignment will remain the same throughout the experiment.

Appendix A provides general maps and layouts of the buildings where the simulation experiment will be conducted.

\subsection{Simulation Environment}

1. The simulation pilots will emulate real world, current, and qualified pilots. Most of the simulation pilots have airline or general aviation pilot experience. You will be given an opportunity to visit the pilot workstation laboratories.

2. VSCS is not available. However, an alternate communications system will emulate similar functionality for the air-to-air, air-to-ground, and ground-to-ground communications.

3. You will be trained on the communications system.

4. The URET version that is used in this experiment is the same that is currently operational at the Memphis Center. URET capabilities are identical. 
5. The DSR version that is used in this experiment is BABO3. The Memphis Center uses $\mathrm{BABO} 4$ version. The differences between these versions are marginal and not significant for this experiment. You will be trained on the differences.

6. There are no ascending and/or descending aircraft in the traffic scenarios. The reason for their exclusion was for complexity issues related to experimental design.

7. Some aircraft targets may unnaturally and unexpectedly appear in handoff status very close to your sector boundary. This is a limitation of the simulation environment.

8. You need to disable the auto hand-off function by entering the $Q A F$ command on DSR. The expert observers will assist you as required.

\subsection{Airspace Description}

1. The airspace of ZME sectors 21 and 20 are combined for the purposes of this experiment. We recognize that they may not be combined for similar air traffic complexity in the real environment.

2. The combined sector altitudes include FL 240 and above. For the purposes of the experiment, the combined sector will be referred to as sector 21 .

3. Sector 44 altitudes include FL240 and above.

4. In addition to sectors 21 and 44 , there will be a sector 78 . Sector 78 emulates a combination of all other sectors that are adjacent to sector 21 and sector 44 . Therefore, if you need to communicate with a sector controller that is not from sector 21 and/or 44, you need to contact sector 78 . 


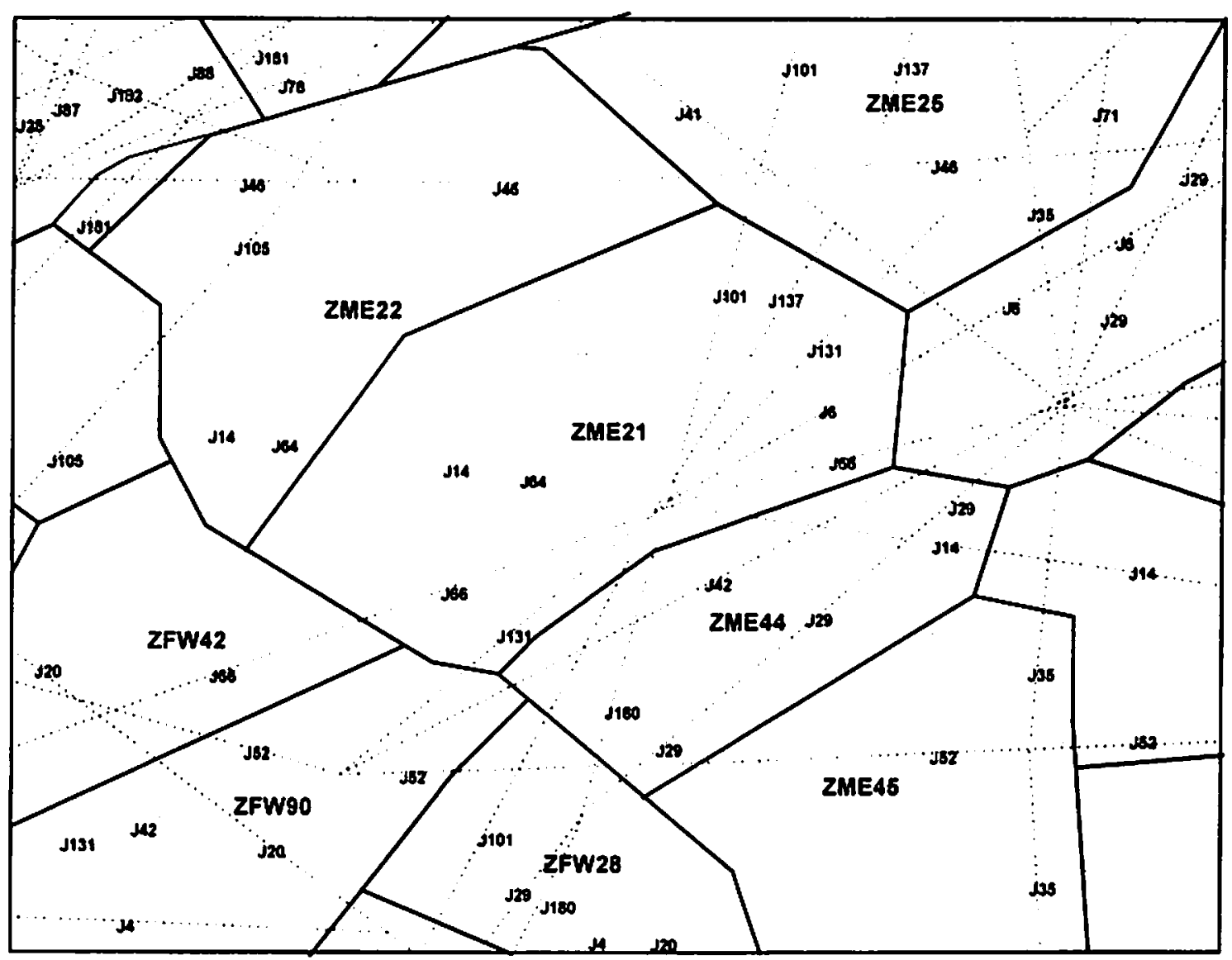

Figure 1. Memphis ARTCC sectors 21 and 44

\section{$5.3 \quad$ Frequencies}

In addition to Sector 21 and 44 frequencies, the experiment emulates an air-to-air frequency. The air-to-air frequency is only available during SS:L1 and SS:L2 conditions. On the air-to-air frequency, the pilots will be able to communicate among themselves. Controllers will be able to selectively monitor the frequency as desired but are not permitted to transmit on the frequency for ANY reason. Table 2 provides the frequencies used in this experiment. 
Table 2. Sector frequencies

\begin{tabular}{|r|l|}
\hline \multicolumn{1}{|c|}{ Type } & \multicolumn{1}{|c|}{ Frequency } \\
\hline Sector 21 & 132.42 \\
\hline Sector 44 & 124.92 \\
\hline Air-to-air & 122.75 \\
\hline Sector 78 & 123.45 \\
\hline
\end{tabular}

6 Data Collection Requirements and Methods

Participants and observer data will be kept locked and secured. No individual names or identities will be recorded or released in any reports.

\subsection{Forms and Questionnaires}

The following table provides a summary of all data collection forms and questionnaires that will be completed by expert observers and/or participant controllers.

Table 3. Forms for Expert Observers and Participant Controllers

\begin{tabular}{|l|l|l|l|}
\hline \multicolumn{1}{|c|}{ Forms } & \multicolumn{1}{|c|}{ Purpose } & \multicolumn{1}{|c|}{ Frequency } & \multicolumn{1}{c|}{ Completed by: } \\
\hline Consent Form & $\begin{array}{l}\text { Consent to voluntary participation in the } \\
\text { study. Understanding of participant rights. }\end{array}$ & $\begin{array}{l}\text { Once prior to } \\
\text { simulation }\end{array}$ & $\begin{array}{l}\text { Controllers \& } \\
\text { Observers }\end{array}$ \\
\hline $\begin{array}{l}\text { Background } \\
\text { Information Form }\end{array}$ & $\begin{array}{l}\text { Provide demographic information related } \\
\text { to professional experience }\end{array}$ & $\begin{array}{l}\text { Once prior to } \\
\text { runs }\end{array}$ & $\begin{array}{l}\text { Controllers \& } \\
\text { Observers }\end{array}$ \\
\hline $\begin{array}{l}\text { During-the-Run } \\
\text { Form }\end{array}$ & $\begin{array}{l}\text { Record critical and interesting events, } \\
\text { controller actions, and observations of the } \\
\text { impact of shared-separation operations. }\end{array}$ & Every run & Observers only \\
\hline Post-Run Form & $\begin{array}{l}\text { Record information concerning overall } \\
\text { workload, situation awareness, the impact } \\
\text { of conflicts, and shared-separation } \\
\text { operations. }\end{array}$ & $\begin{array}{l}\text { End of every } \\
\text { run }\end{array}$ & $\begin{array}{l}\text { Controllers \& } \\
\text { Observers }\end{array}$ \\
\hline $\begin{array}{l}\text { Post-Simulation } \\
\text { Form }\end{array}$ & $\begin{array}{l}\text { Respond to general questions about shared- } \\
\text { separation operations and the overall } \\
\text { experiment. }\end{array}$ & $\begin{array}{l}\text { Once at the } \\
\text { end of } \\
\text { simulation }\end{array}$ & $\begin{array}{l}\text { Controllers \& } \\
\text { Observers }\end{array}$ \\
\hline
\end{tabular}

\subsection{Workload Assessment Keypad (WAK) Ratings}

You will be asked to record a measure of your overall workload every 5 minutes on the WAK. The WAK will illuminate and emit a low-level beep every 5-minutes. At those cues, press the key that corresponds to your workload level. 
While reporting your workload rating, please consider combined mental and physical workload. Your rating should reflect an instantaneous measure. In other words, rate your overall workload at that moment, not as a cumulative measure of the last 5 minutes. The workload rating scale is shown in Table 4.

Table 4. Workload Rating Scale

\begin{tabular}{|ccccc|}
\hline 1 & 2 & 3 & 4 & 5 \\
Very Low & Moderate & & Very High \\
\hline
\end{tabular}

\subsection{Additional points/events of interest}

In addition to the 5-minute workload ratings, you will be asked to identify and report additional points during the scenarios. These points are defined as follows:

- Point A - When you detect a potential conflict between a pair of aircraft with a reasonable certainty. (When you begin to pay more attention to a pair of aircraft due to the possibility of conflict). Identify for all scenarios.

- Point B - When you would take action to resolve a potential conflict under current operating rules (as described in 7110.65). Assume that pilots do not have CDTI-AL in their cockpits. Identify for SS:L1 and SS:L2 only.

- Point C - When you would take an action to resolve a potential conflict under free flight conditions where pilots are self-separating themselves. Assume that pilots have CDTI-AL in their cockpits. Identify for SS:L2 only.

Table 5. WAK and Additional Data Collection Summary

\begin{tabular}{|c|c|c|c|c|}
\hline Scenario & $\begin{array}{l}\text { WAK (every 5 } \\
\text { minutes) }\end{array}$ & Point A & Point B & Point C \\
\hline CO & $\sqrt{ }$ & $\sqrt{ }$ & & \\
\hline CO:CDTI & $\sqrt{ }$ & $\checkmark$ & & \\
\hline SS:LL & $\sqrt{ }$ & $\checkmark$ & $\checkmark$ & \\
\hline SS:L2 & $\sqrt{ }$ & $\sqrt{ }$ & $\sqrt{ }$ & $\checkmark$ \\
\hline
\end{tabular}

The controller (either R or D) who first identifies a point of interest (Point A, B, or C) will inform the expert observer. The same controller will point out the aircraft pair to the expert observer. The expert observer will then document the aircraft pair, simulation time, and the point of interest. The observer will also record if point A was identified using URET. 
Appendix.C

Controller and Expert Observer Forms 


\section{Air-Ground Integration Experiment (AGIE) \\ Background Information Form}

This form is to be completed by all AGIE Controllerand Expert Observer participants. This form requests general background information.

Your name will not be listed or appear in any reports to ensure your anonymity and to encourage unbiased reporting. Findings will be reported generically (Controller or Observer A, B, C, etc.). 


\section{Air-Ground Integration Experiment (AGIE) \\ Background Information Form}

Controller or Observer (circle one)

Date: 1

ID\#:

Male or Female (circle one)

1. What is your age?

Years:

2. What is your total experience as an air traffic control specialist (include FAA and military experience)?

Years: Months:

3. How long have you actively controlled traffic for the FAA?

Years: Months:

4. How long have you been a Full Performance Level (FPL) controller?

Years: Months:

5. How long have you been a controller at Memphis?

Years: Months:

6. How long have you been using DSR?

Years: Months: or Not Trained

7. How long have you been using URET? Years: Months: or Not Trained

8. What is the percentage of time you use URET?

Sector 21: Sector 44:

Overall: 


\section{Air-Ground Integration Experiment (AGIE) Observer Post-Run Form}

This form is to be completed by observers participating in AGIE and requests information regarding your overall observations and judgments during the run.

Your name will not be listed or appear in any reports to ensure your anonymity and to encourage unbiased reporting. Findings will be reported generically (Observer A, B, C, etc.).

You are encouraged to write any additional comments that you feel are important.

When making your ratings, please consider all levels of the scale. You are encouraged to write any additional comments you feel are important. 


\section{Air-Ground Integration Experiment (AGIE) Observer Post-Run Form}

Observer ID\#:

Controller IDs, R-Side:

D-Side:

Scenario:

Run\#:

Date:

Sector:

Where applicable, please circle the most accurate response.

The term physical taskload refers to the physical activities associated with accomplishing tasks. For example, performing the physical actions associated with entering keyboard data, communications, manipulating the trackball are components of physical taskload.

1. Circle the response that describes the controller physical taskload during the simulation run.

\begin{tabular}{|c|ccccc|}
\hline R-Controller & $\begin{array}{c}1 \\
\text { Very Low }\end{array}$ & 2 & $\begin{array}{c}3 \\
\text { Moderate }\end{array}$ & 4 & $\begin{array}{c}5 \\
\text { Very High }\end{array}$ \\
\hline D-Controller & 1 & 2 & $\begin{array}{c}3 \\
\text { Moderate }\end{array}$ & 4 & $\begin{array}{c}5 \\
\text { Very High }\end{array}$ \\
\hline
\end{tabular}

Comments.

2. Identify factors that interfered with maintaining separation. 
3. Identify any aircraft separation issues that became apparent.

4. Identify any ground-to-ground coordination / communications issues that became apparent.

5. Identify any coordination / communications issues between R-side and D-side that became apparent.

6. Identify any ground-to-air communications issues that became apparent.

7. In your opinion, how much vertical and horizontal separation represents effective safety margin?

Vertical separation (below FL290):

Vertical separation (above FL290):

Horizontal separation:

(ft.)

(ft.)

(nm) 
8. Comment on any other issues that you observed during this run that could help the researchers understand the events as they occurred. 


\section{Air-Ground Integration Experiment (AGIE) Controller Exit Form}

This is the final form to be completed by air traffic controllers participating in AGIE and requests information regarding your overall experiences and judgments about the simulations that you just completed.

Your name will not be listed or appear in any reports to ensure your anonymity and to encourage unbiased reporting. Findings will be reported generically (Controller A, B, C, etc.).

When making your ratings, please consider all levels of the scale. You are encouraged to write any additional comments you feel are important. 


\section{Air-Ground Integration Experiment (AGIE) \\ Controller Exit Form}

Controller ID\#:

Position: R-Side or D-Side (circle one)

Scenario:

Run\#:

Date:

Sector:

Where applicable, please circle the most accurate response.

1. What additional procedures would you suggest to facilitate the implementation of sharedseparation operations?

2. For each control condition, what additional information and decision support tools would help you under the following operational conditions please rate the effectiveness of the support tool (i.e., URET) for conducting air traffic operations.

\begin{tabular}{|l|l|}
\hline $\begin{array}{l}\text { Current } \\
\text { operations (CO) } \\
\text { - controller has } \\
\text { full authority }\end{array}$ & \\
\hline $\begin{array}{l}\text { Current } \\
\text { operations with } \\
\text { CDTI } \\
\text { (CO:CDTI) - } \\
\text { controller has } \\
\text { full authority }\end{array}$ & \\
\hline $\begin{array}{l}\text { Shared- } \\
\text { separation } \\
\text { authority }\end{array}$ & \\
(SS:LI) & \\
\hline
\end{tabular}


Flight deck has

full separation

authority

(SS:L2)

3. What additional information (or tools) would be useful for shared-separation operations?

4. Rate the realism of the simulated flight crew responses compared to your field experience.
1
2
3
4
5
Very
Moderate
Very Realistic

Unrealistic

Comments.

5. Rate the overall realism of the simulation compared to your field experience.
1
Very
2
3
Moderate
4
5
Unrealistic

Comments.

6. Rate the adequacy of the simulation training.
1
2
3
Moderate
4
5
Adequate

Comments.

7. Were you anytime confused about who has the separation responsibility and what your role is? YES NO If Yes, please explain 
8. Did you find scenarios similar (except for training)? YES

NO If YES, describe how.

9. Were you able to keep up with providing three data points A, B, C under SS:L1 and SS:L2 conditions? YES NO, If NO, describe below under which locus it was difficult?

10. What can be done to improve simulation fidelity? If we were to conduct future sharedseparation related research, what improvements in scenario, traffic, phraseology, and simulation would you suggest? 


\section{Air-Ground Integration Experiment (AGIE) \\ Controller Post-Run Form (CO)}

This form is to be completed by air traffic controllers participating in AGIE and requests information regarding your overall experiences and judgments about the run.

Your name will not be listed or appear in any reports to ensure your anonymity and to encourage unbiased reporting. Findings will be reported generically (Controller $A, B, C$, etc.).

When making your ratings, please consider all levels of the scale. You are encouraged to write any additional comments you feel are important. 


\section{Air-Ground Integration Experiment (AGIE) Controller Post-Run Form (CO)}

Controller ID\#:

Position: R-Side or D-Side (circle one)

Scenario:

Run\#:

Date:

Sector:

Where applicable, please circle the most accurate response.

1. Rate your physical workload level (e.g., data entry, coordination, communications, etc.) during this run. 1

Very Low

23

Moderate

4 5

Comments.

2. Rate your mental workload level (e.g., planning, predicting, monitoring, etc.) during this run.

1

Very Low

$2 \quad 3$

Moderate

4

5

Very High

\section{Comments.}

3. Rate your overall workload level (physical and mental combined) during this run.
1
Very Low
2
3
Moderate
5
Very High

Comments.

4. Identify events that significantly affected your overall workload during the run. 
5. Rate the overall workload associated with maintaining aircraft separation. (e.g., monitoring and planning).
1
2
3
Moderate
4
5
Very High

Very Low

Comments.

6. Rate the overall workload associated with maintaining ground-to-ground (i.e., land line) coordination / communications. 1
Very Low
2
3
Moderate
4
5
Very High

Comments.

7. Rate the overall workload associated with maintaining coordination / communications between $\mathrm{R}$-side and $\mathrm{D}$-side.
1
Very Low
2
3
Moderate
4
5
Very High

Comments.

8. Rate the overall workload associated with maintaining ground-to-air communications. 1
Very Low
2
3
Moderate
4
5
Very High

\section{Comments.}

9. Rate the overall workload associated with coordinating URET alerts to adjacent sectors.

$\begin{array}{ccccc}1 & 2 & 3 & 4 & 5 \\ \text { Very Low } & & \text { Moderate } & & \text { Very High }\end{array}$

Comments. 
10. Rate the amount of time available to assure safe separation during peak traffic periods.

1

Too Little

Comments.
2

Adequate
4

5

Too Much

11. Rate the amount of time available to complete all required coordination during peak traffic periods.

1

Too Little

Comments.
2

Adequate
4

5 Too Much

12. During the peak traffic period, I felt rushed.

1

Not at All

Comments.
2

About Half of

the Time
4

5

All of the

Time

13. During the peak traffic period, I felt bored.

1

Not at All

Comments.
3

About Half of

the Time
4 5

All of the

Time

14. Compared to current operations the procedures used in this session resulted in a level of safety that was:

1

Compromised

If enhanced, why?
3

Unchanged
4

5

Enhanced

If compromised, why? 
The term overall situation awareness refers to what is commonly known as the controller's "picture" and involves processing the relevant air traffic information to develop a thorough understanding of the current situation that facilitates appropriate air traffic actions in a timely manner.

15. Rate your level of overall situation awareness during this run.

$\begin{array}{ccccc}1 & 2 & 3 & 4 & 5 \\ \text { Moderate } & \text { Mow } & & & \text { Very High }\end{array}$
Comments.

16. Rate the amount of the information available to identify and resolve conflicts.

1

Too Little

Comments.
2

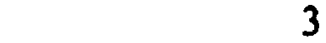

Adequate
5

Too Much

- What additional information is needed, if any?

- What information was not useful or added clutter, if any?

17. How timely were the conflict probe alerts?

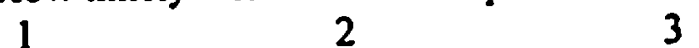

Too Early

Comments.

Adequate

4

5

Too Late

18. Identify factors that interfered with maintaining separation.

19. Identify any changes in your control strategies for this run. 
20. In your opinion, how much vertical and horizontal separation represents effective safety margin?

Vertical separation (below FL290):

Vertical separation (above FL290):

(ft)

Horizontal separation:

(f)

$(\mathrm{nm})$

21. Please provide any additional comments or concerns you may have about this run. 


\section{Air-Ground Integration Experiment (AGIE) \\ Controller Post-Run Form (CO:CDTI)}

This form is to be completed by air traffic controllers participating in AGIE and requests information regarding your overall experiences and judgments about the run.

Your name will not be listed or appear in any reports to ensure your anonymity and to encourage unbiased reporting. Findings will be reported generically (Controller A, B, C, etc.).

When making your ratings, please consider all levels of the scale. You are encouraged to write any additional comments you feel are important. 


\section{Air-Ground Integration Experiment (AGIE) Controller Post-Run Form (CO:CDT)}

Controller ID\#:

Position: R-Side or D-Side (circle one)

Scenario:

Run\#:

Sector:

Date: $\square$

Where applicable, please circle the most accurate response.

1. Rate your physical workload level (e.g., data entry, coordination, communications, etc.) during this run.

123

Very Low

Moderate

4

5

Comments.

2. Rate your mental workload level (e.g., planning, predicting, monitoring, etc.) during this run.

$\begin{array}{ccccc}1 & 2 & 3 & 4 & 5 \\ \text { Very Low } & & \text { Moderate } & & \text { Very High }\end{array}$

Comments.

3. Rate your overall workload level (physical and mental combined) during this run. $\begin{array}{llll}1 & 2 & 3 & 4\end{array}$

Very Low

Moderate

45

Very High

Comments.

4. Identify events that significantly affected your overall workload during the run. 
5. Rate the overall workload associated with maintaining aircraft separation. (e.g., monitoring and planning).
1
2
3
Moderate
4
5
Very High

Very Low

Comments.

6. Rate the overall workload associated with maintaining ground-to-ground (i.e., land line) coordination/communications.
1
Very Low
2
3
Moderate
4
5
Very High

Comments.

7. Rate the overall workload associated with maintaining coordination/communications between $\mathrm{R}$-side and D-side.
1
2
3
4
5
Very High

Very Low

Comments.

8. Rate the overall workload associated with maintaining ground-to-air communications.
1
Very Low
2
3
Moderate
4
5
Very High

Comments.

9. Rate the overall workload associated with coordinating URET alerts to adjacent sectors.

$\begin{array}{ccccc}\text { 9. Rate the overall workload associated with coordinating } & 3 & 5 \\ 1 & 2 & 3 & 4 & \text { Very High }\end{array}$

Comments. 
10. Rate the amount of time available to assure safe separation during peak traffic periods.

Too Little

2

3

Adequate
4

5

Too Much Comments.

11. Rate the amount of time available to complete all required coordination during peak traffic periods.

1

Too Little

Comments.
2

Adequate
4 5

Too Much

12. During the peak traffic period, I felt rushed:

1

Not at All

Comments.

\section{2}

About Half of

the Time
4 5

All of the

Time

13. During the peak traffic period, I felt bored:

123

Not at All

Comments.
About Half of the Time
4 5

All of the

Time

14. Compared to current operations the procedures used in this session resulted in a level of safety that was:

$1 \quad 2 \quad 3$

Compromised

If enhanced, why?

Unchanged

4 5

Enhanced

If compromised, why? 
The term overall situation awareness refers to what is commonly known as the controller's "picture" and involves processing the relevant air traffic information to develop a thorough understanding of the current situation that facilitates appropriate air traffic actions in a timely manner.

15. Rate your level of overall situation awareness during this run.
1
2
3
4
5
Moderate
Very High

Very Low

Comments.

16. Rate the amount of the information available to identify and resolve conflicts.

1

Too Little

Comments.
2

Adequate
4

5

Too Much

- What additional information is needed, if any?

- What information was not useful or added clutter, if any?

17. How timely were the conflict probe alerts?

123

Too Early

Comments.
4 5

Too Late

18. Identify factors that interfered with maintaining separation.

19. Identify any changes in your control strategies for this run. 
20. In your opinion, how much vertical and horizontal separation represents effective safety margin?

Vertical separation (below FL290):

Vertical separation (above FL290):

Horizontal separation:

(ft.)

(ft.)

$(\mathrm{nm})$

21. Please provide any additional comments or concerns you may have about this run. 


\section{Air-Ground Integration Experiment (AGIE) Controller Post-Run Form (SS:L1)}

This form is to be completed by air traffic controllers participating in AGIE and requests information regarding your overall experiences and judgments about the run.

Your name will not be listed or appear in any reports to ensure your anonymity and to encourage unbiased reporting. Findings will be reported generically (Controller $A, B, C$, etc.).

When making your ratings, please consider all levels of the scale. You are encouraged to write any additional comments you feel are important. 


\section{Air-Ground Integration Experiment (AGIE) Controller Post-Run Form (SS:L1)}

Controller ID\#:

Position:

R-Side or D-Side (circle one)

Scenario:

Run\#:

Date:

Where applicable, please circle the most accurate response.

1. What changes would you recommend for the implementation of Free Flight as simulated in this study?
Sector:

Automation.

Procedures.

Other. 
2. Rate your physical workload level (e.g., data entry, coordination, communications, etc.) during this run.

123

Very Low

Comments.

Moderate

4 5

Very High

3. Rate your mental workload level (e.g., planning, predicting, monitoring, etc.) during this run.

$\begin{array}{ccccc}1 & 2 & 3 & 4 & 5 \\ \text { Very Low } & \text { Moderate } & & \text { Very High }\end{array}$

Comments.

4. Rate your overall workload level (physical and mental combined) during this run.

$\begin{array}{ccccc}1 & 2 & 3 & 4 & 5 \\ \text { Very Low } & & \text { Moderate } & & \text { Very High }\end{array}$

Comments.

5. Identify events that significantly affected your overall workload during the nun.

6. Rate the overall workload associated with maintaining aircraft separation. (e.g., monitoring and planning).

$1 \quad 2 \quad 3$

Very Low

Comments.

Moderate

4

5

Very High 
7. Rate the overall workload associated with maintaining ground-to-ground (i.e., land line) coordination / communications.

123

Very Low

Comments.

Moderate

4 5 Very High

8. Rate the overall workload associated with maintaining coordination/communications between R-side and D-side.

1

Very Low

Comments.

\section{2}

Moderate
4 5

9. Rate the overall workload associated with maintaining ground-to-air communications.

$\begin{array}{ccccc}1 & 2 & 3 & 4 & 5 \\ \text { Very Low } & 2 & \text { Moderate } & & \text { Very High }\end{array}$
Comments.

10. Rate the overall workload associated with coordinating URET alerts to adjacent sectors.

$\begin{array}{ccccc}1 & 2 & 3 & 4 & 5 \\ \text { Very Low } & \text { Moderate } & & \text { Very High }\end{array}$
Comments.

11. Rate the amount of time available to assure safe separation during peak traffic periods.

Too Little

2 Comments.
4

Adequate
5

Too Much 
12. Rate the amount of time available to complete all required coordination during peak traffic periods.

1

Too Little

Comments.
2

3

Adequate
4

5

Too Much

13. During the peak traffic period, I felt rushed.

1

Not at All
2

3

About Half of

the Time
4

All of the

Time

Comments.

14. During the peak traffic period, I felt bored.

1

Not at All

2

2

3

About Half of

the Time
4 5

All of the

Time

\section{Comments.}

15. Compared to current operations the procedures used in this session resulted in a level of safety that was:

1

Compromised

If enhanced, why?.
2

3

Unchanged
4 5 Enhanced

If compromised, why? 
The term overall situation awareness refers to what is commonly known as the controller's "picture" and involves processing the relevant air traffic information to develop a thorough understanding of the current situation that facilitates appropriate air traffic actions in a timely manner.

16. Rate your level of overall situation awareness during this run.

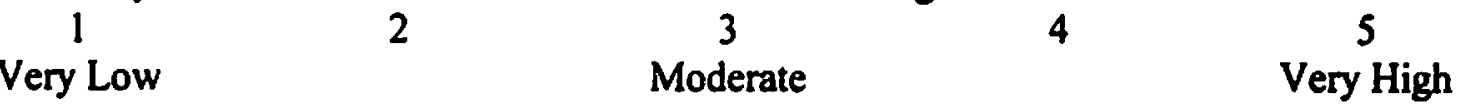
Comments.

17. Rate the amount of the information available to identify and resolve conflicts.

1

Too Little

Comments.
2

2

3

Adequate
4

5

Too Much

- What additional information is needed, if any?

-What information was not useful or added clutter, if any?

18. How timely were the conflict probe alerts?

123

Too Early

Comments.

Adequate

4 5

Too Late

19. Identify factors that interfered with maintaining separation.

20. Identify any changes in your control strategies for this run. 
21. In your opinion, how much vertical and horizontal separation represents effective safety margin?

Vertical separation (below FL290):

Vertical separation (above FL290):

Horizontal separation:

$(\mathrm{nm})$

22. Rate how often you monitored air-to-air communications.

$\begin{array}{ccccc}1 & 3 & 4 & 5 \\ \begin{array}{c}\text { Never } \\ \text { ments. }\end{array} & 2 & \text { Sometimes } & & \text { Always }\end{array}$

Comments.

23. Rate the usefulness of monitoring air-to-air communications.

$\begin{array}{cccc}1 & 2 & 3 & 5 \\ \text { Not Useful } & \text { Useful } & 4 & \text { Very Useful }\end{array}$

Comments.

24. Rate the helpfulness of the shared-separation operations concept for performing your job.

123

Not Helpful

Comments.
Moderately

Helpful
5

Very Helpful

25. If you cancelled Free Flight, identify the reasons.

- If you delayed in resuming Free Flight, identify the reasons. 
26. Please provide any additional comments or concerns you may have about this run.

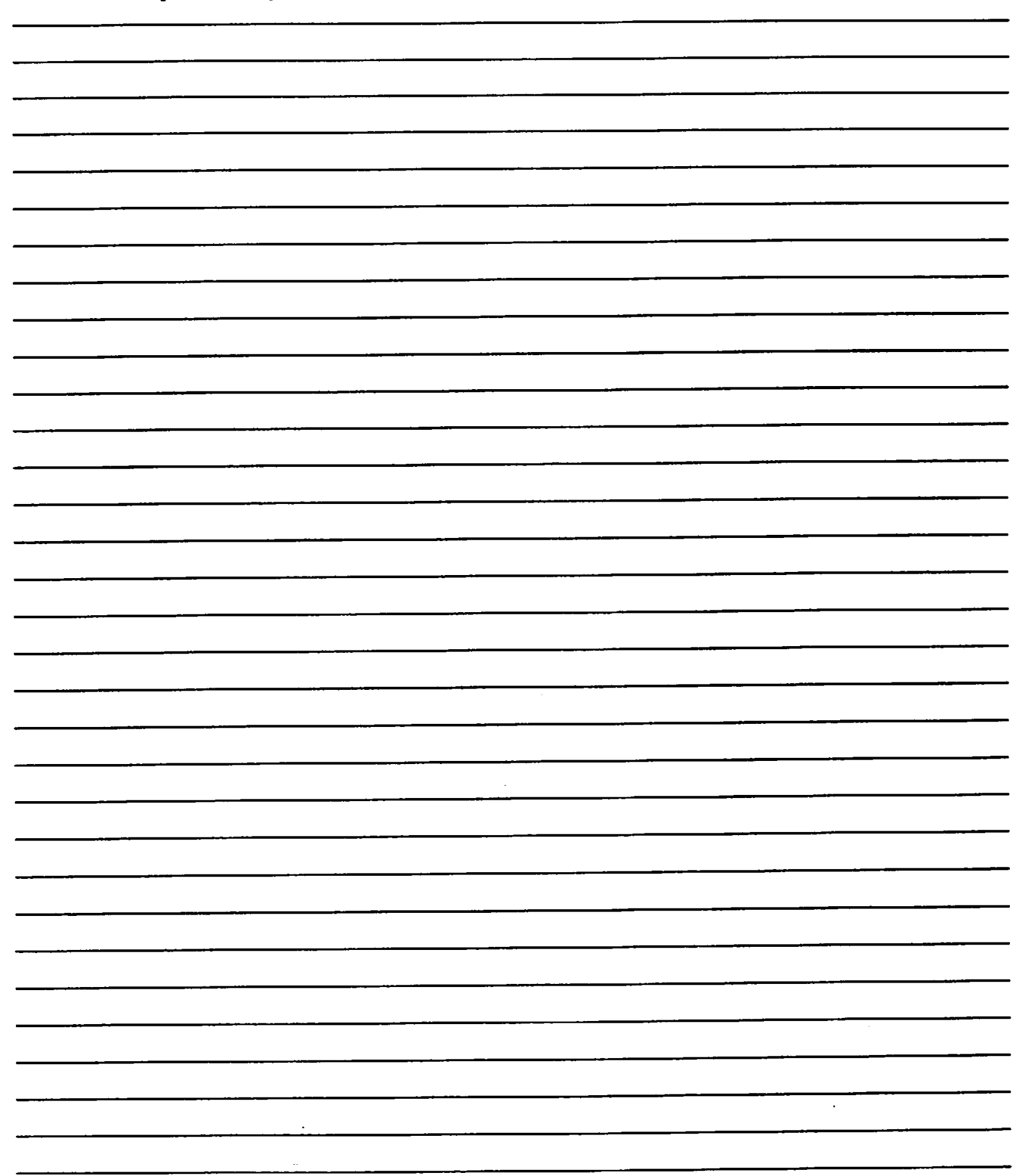




\section{Air-Ground Integration Experiment (AGIE) \\ Controller Post-Run Form (SS:L2)}

This form is to be completed by air traffic controllers participating in AGIE and requests information regarding your overall experiences and judgments about the run.

Your name will not be listed or appear in any reports to ensure your anonymity and to encourage unbiased reporting. Findings will be reported generically (Controller $A, B, C$, etc.).

When making your ratings, please consider all levels of the scale. You are encouraged to write any additional comments you feel are important. 


\section{Air-Ground Integration Experiment (AGIE) \\ Controller Post-Run Form (SS:L2)}

Controller IDH:

Position: R-Side or D-Side (circle one)

Scenario:

Run\#:

Date:

Sector:

Where applicable, please circle the most accurate response.

1. What changes would you recommend for the implementation of Free Flight as simulated in this study?

Automation.

Procedures.

Other. 
2. Rate your physical workload level (e.g., data entry, coordination, communications, etc.) during this run.
1
2
3
Moderate
4
5
Very High

Very Low

Comments.

3. Rate your mental workload level (e.g., planning, predicting, monitoring, etc.) during this run.

1

Very Low

2

3

Moderate

4

5

Comments.

4. Rate your overall workload level (physical and mental combined) during this run.

$\begin{array}{ccccc}1 & 3 & 4 & 5 \\ \text { Very Low } & 2 & \text { Moderate } & & \text { Very High }\end{array}$

\section{Comments.}

5. Identify events that significantly affected your overall workload during the nun.

6. Rate the overall workload associated with maintaining aircraft separation. (e.g., monitoring and planning).

1

Very Low

Comments.
3

Moderate
4

5

Very High 
7. Rate the overall workload associated with maintaining ground-to-ground (i.e., land line) coordination/communications.

$\begin{array}{ccccc}1 & 2 & 3 & 4 & 5 \\ \text { Very Low } & \text { Moderate } & & \text { Very High } \\ \text { Comments. } & & & \end{array}$

8. Rate the overall workload associated with maintaining coordination/communications between $\mathrm{R}$-side and D-side.
Very Low
2
3
Moderate
4
5
Very High

Comments.

9. Rate the overall workload associated with maintaining ground-to-air communications.

1

Very Low

Comments.
2

Moderate
4

5

Very High

10. Rate the overall workload associated with coordinating URET alerts to adjacent sectors.

1

Very Low

Comments.
2

3

Moderate
4

5

Very High

11. Rate the amount of time available to assure safe separation during peak traffic periods.

\section{1}

Too Little Comments.
2 3

Adequate
4

5 Too Much 
12. Rate the amount of time available to complete all required coordination during peak traffic periods.

123

Too Little Comments.

Adequate

4 5 Too Much

13. During the peak traffic period, I felt rushed.

1

Not at All
2

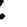

(1)

3

About Half of the Time
4 5 All of the

Time

Comments.

14. During the peak traffic period, I felt bored. 1

Not at All
2

3
$\begin{gathered}\text { About Half of } \\ \text { the Time }\end{gathered}$

4 5

All of the

Time

Comments.

15. Compared to current operations the procedures used in this session resulted in a level of safety that was:

1

2

3

Unchanged

4

5

Compromised

If enhanced, why?

If compromised, why? 
The term overall situation awareness refers to what is commonly known as the controller's "picture" and involves processing the relevant air traffic information to develop a thorough understanding of the current situation that facilitates appropriate air traffic actions in a timely manner.

16. Rate your level of overall situation awareness during this run.

$\begin{array}{ccccc}1 & 2 & 3 & 4 & 5 \\ \text { Very Low } & \text { Moderate } & & \text { Very High }\end{array}$
Comments.

17. Rate the amount of the information available to identify and resolve conflicts.

$$
1
$$

Too Little

Comments.
2

(dequ

Adequate
4

Too Much

- What additional information is needed, if any?

- What information was not useful or added clutter, if any?

18. How timely were the conflict probe alerts?

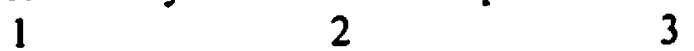

Too Early

Comments.

Adequate

4 5

Too Late

19. Identify factors that interfered with maintaining separation.

20. Identify any changes in your control strategies for this run. 
21. In your opinion, how much vertical and horizontal separation represents effective safety margin?

Vertical separation (below FL290):

Vertical separation (above FL290):

Horizontal separation:

$(\mathrm{nm})$

22. Rate how often you monitored air-to-air communications.
1
Never
2
3
Sometimes
5
Always

Comments.

23. Rate the usefulness of monitoring air-to-air communications.

1

Not Useful

2

3

Moderately

Useful
4 Very Useful

Comments.

24. Rate the helpfulness of the shared-separation operations concept for performing your job.

Not Helpful

2

Comments.

\section{Comments.}

3

Moderately

Helpful
4

Very Helpful 
25. Please provide any additional comments or concerns you may have about this run.

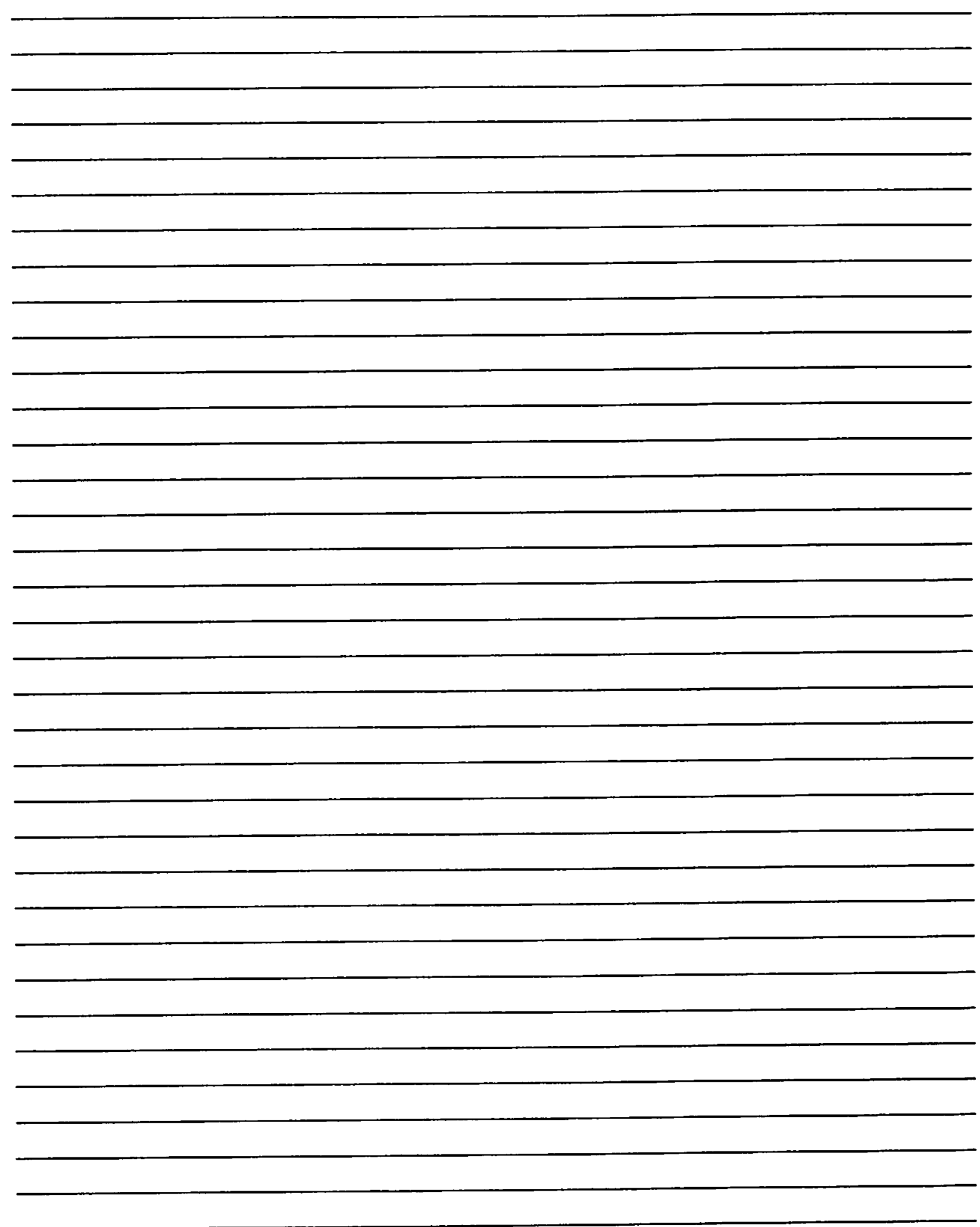




\section{Air-Ground Integration Experiment (AGIE) Observer During-the-Run Form}

This form is to be completed by observers participating in AGIE and requests information regarding your overall observations and judgments during the run.

Your name will not be listed or appear in any reports to ensure your anonymity and to encourage unbiased reporting. Findings will be reported generically (Observer A, B, C, etc.).

You are encouraged to write any additional comments that you feel are important.

Please review the items contained in the questionnaire before the simulation strts. Listen and closely observe the actions of the controller team operating in your sector(s). Based on what you hear and see, apply your expertise to provide information and comments that you feel may be valuable to this study. 


\section{AGIE -Observer During-the-Run Form}

Observer ID\#:

Scenario:

Controller IDs, R-Side:

D-Side:

Run \#:

Record of Conflict Detection and Resolution Points

\begin{tabular}{|c|c|c|c|c|c|c|}
\hline URET & Point A & Point B & Point C & Aircraft 1 ID & Aircraft 2 ID & Comments \\
\hline $\bar{U}$ & $\bar{A}$ & B & $\bar{C}$ & & & \\
\hline \multicolumn{7}{|l|}{ Sim Time } \\
\hline $\bar{U}$ & $\bar{A}$ & B & C & & & \\
\hline \multicolumn{7}{|l|}{ Sim Time } \\
\hline $\bar{U}$ & $\overline{\mathbf{A}}$ & $\bar{B}$ & C & & & \\
\hline \multicolumn{7}{|l|}{ Sim Time } \\
\hline $\bar{U}$ & $\mathbf{A}$ & B & $\bar{C}$ & & & \\
\hline \multicolumn{7}{|l|}{ Sim Time } \\
\hline $\mathbf{U}$ & $\bar{A}$ & $\bar{B}$ & C & & & \\
\hline \multicolumn{7}{|l|}{ Sim Time } \\
\hline $\bar{U}$ & $\bar{A}$ & B & $\bar{C}$ & & & \\
\hline \multicolumn{7}{|l|}{ Sim Time } \\
\hline $\bar{U}$ & $\bar{A}$ & $\bar{B}$ & c & & & \\
\hline \multicolumn{7}{|l|}{ Sim Time } \\
\hline $\bar{U}$ & $\bar{A}$ & B & c & & & \\
\hline \multicolumn{7}{|l|}{ Sim Time } \\
\hline $\bar{U}$ & $\bar{A}$ & B & C & & & \\
\hline Sim Time & & & & & & \\
\hline
\end{tabular}

Free Flight Cancellations (Only for SS:L1 and SS:L2)

\begin{tabular}{|c|c|c|c|c|c|}
\hline Aircraft IDs & $\begin{array}{l}\text { Simulation Tim } \\
\text { of Free Flight } \\
\text { Cancellation }\end{array}$ & $\begin{array}{c}\text { Simulation Time } \\
\text { that Free Flight } \\
\text { Resumed }\end{array}$ & $\begin{aligned} \text { Free } \\
\text { Cancel } \\
\text { Pilc } \\
\text { Cont }\end{aligned}$ & $\begin{array}{l}\text { light } \\
\text { ed by: } \\
\text { or } \\
\text { oller }\end{array}$ & Why was Free Flight Cancelled? \\
\hline & & & & C & \\
\hline & & & $\overline{\mathbf{P}}$ & $\bar{C}$ & \\
\hline & & & $\overline{\mathbf{P}}$ & $\bar{C}$ & \\
\hline & & & $\mathbf{P}$ & C & \\
\hline & & & $\mathrm{P}$ & $\mathrm{C}$ & \\
\hline & & & $\mathbf{P}$ & $\bar{C}$ & \\
\hline
\end{tabular}

Record of Situation Awareness, Separation Errors \& Airspace Violations Events 
Please record the time of the following events as and when you notice (record the time of the event, aircraft ID, and put a check mark in the appropriate column)

\begin{tabular}{|c|c|c|c|c|c|c|c|}
\hline $\begin{array}{c}\text { Simulation } \\
\text { Time }\end{array}$ & Aircraft ID & $\begin{array}{c}\text { Separation } \\
\text { Error }\end{array}$ & $\begin{array}{c}\text { Airspace } \\
\text { Violations }\end{array}$ & $\begin{array}{c}\text { Missed } \\
\text { Handofis }\end{array}$ & $\begin{array}{l}\text { Late } \\
\text { Recognition of } \\
\text { Conflicts }\end{array}$ & $\begin{array}{c}\text { Failure to } \\
\text { Correct } \\
\text { Readback } \\
\text { Errors }\end{array}$ & Others (Specify) \\
\hline & & & & & & & \\
\hline & & & & & & & \\
\hline & & & & & & & \\
\hline & & & & & & & \\
\hline & & & & & & & \\
\hline & & & & & & & \\
\hline & & & & & & & \\
\hline & & & & & & & \\
\hline & & & & & & & \\
\hline & & & & & & & \\
\hline & & & & & & & \\
\hline & & & & & & & \\
\hline & & & & & & & \\
\hline & & & & & & & \\
\hline & & & & & & & \\
\hline & & & & & & & \\
\hline & & & & & & & \\
\hline & & & & & & & \\
\hline & & & & & & & \\
\hline & & & & & & & \\
\hline & & & & & & & \\
\hline
\end{tabular}


As and when appropriate, please record your observations regarding the following:

1. Use of URET

2. Coordination between $\mathrm{R}$ and $\mathrm{D}$ side controllers

3. Coordination with other sectors

4. Phraseology

5. Difficulty with simulation environment, equipment, voice system, etc.

6. Air-to-air frequency usage (for SS:LL and SS:L2 only)

7. Other relevant information 


\section{Appendix D \\ Human Research Minimal Risk Consent}




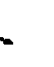

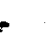$$
\text { . }
$$ 
NASA Ames Research

HUMAN RESEARCH

MINIMAL RISK CONSENT

To the Test Subject: Please read this consent form and the attached protocol and/or subject instructions carefully. Make sure all your questions have been answered to your satisfaction before signing.

A. I agree to participate as a subject in the research experiment as described in the attached protocol or subject instructions. I understand that I am employed by who can be contacted at

B. I understand that my participation could cause me minimal risk*, inconvenience, or discomfort. The purpose and procedures have been explained to me and I understand the risks and discomforts as described in the attached research protocol.

C. To my knowledge, I have no medical conditions, including pregnancy, that will prevent my participation in this study. I understand that if my medical status should change while a participant in the research experiment that there may be unforeseeable risks to me (or the embryo or fetus if applicable). I agree to notify the Principal Investigator (P.I.) or medical monitor of any known changes in my condition for safety purposes.

D. My consent to participate as a subject has been freely given. I may withdraw my consent, and thereby withdraw from the study at any time without penalty or loss of benefits to which I am entitled. I understand that the P.I. may request my withdrawal or the study may be terminated for any reason. I agree to follow procedures for orderly and safe termination.

E. I am not releasing NASA from liability for any injury arising as a result from my participation in this study.

F. I hereby agree that all records collected by NASA in the course of this study are available to the research study investigators, support staff, and any duly authorized research review committee. I grant NASA permission to reproduce and publish all records, notes, or data collected from my participation, provided there is no association of my name with the collected data and that confidentiality is maintained, unless specifically waived by me.

G. I have had an opportunity to ask questions and have received satisfactory answers to all my questions. I understand the P.I. for the study is the person responsible for this activity and that any pertinent questions regarding the research will be addressed to him/her during the course of the study. I have read the above agreement, the attached protocol and/or subject instructions prior to signature, and understand the contents. 


\begin{tabular}{|ll|}
\hline $\begin{array}{l}\text { Minimal Risk means that the probability and magnitude of harm or discomfort } \\
\text { anticipated in the research are not greater, in and of themselves, than those ordinarily } \\
\text { encountered in daily life or during the performance of routine physical or } \\
\text { psychological examinations or tests. }\end{array}$ \\
$\begin{array}{ll}\text { Signature of Test Subject } \\
\text { Date }\end{array}$ & $\begin{array}{l}\text { Signature of Principal Investigator } \\
\text { Date }\end{array}$ \\
$\begin{array}{ll}\text { Printed/Typed Name of Test } \\
\text { Subject/Evaluation Pilot }\end{array}$ & $\begin{array}{l}\text { Printed/Typed Name of Principal } \\
\text { Investigator }\end{array}$ \\
\hline Address & $\begin{array}{l}\text { Telephone Number of Principal } \\
\text { Investigator }\end{array}$ \\
& \\
\hline City, State, Zip Code & $\begin{array}{l}\text { Subject Signature: Authorization for } \\
\text { Videotaping }\end{array}$ \\
& \\
\hline Telephone Number of Test Subject & $\begin{array}{l}\text { Subject Signature: Authorization for } \\
\text { Release of Information to Non-NASA } \\
\text { Source(s) }\end{array}$ \\
\hline
\end{tabular}


Appendix E

Pilot Daily Schedule 


$$
\text { ' }
$$
. 
Daily Schedule for Pilot Participants

\begin{tabular}{|c|c|c|c|c|}
\hline Time (PST) & Day 2 & Day 3 & Day 4 & Day 5 \\
\hline $\begin{array}{c}\text { 09:00am } \\
: 15\end{array}$ & \multirow{17}{*}{$\begin{array}{l}\text { Pilot } \\
\text { Travel }\end{array}$} & \multirow{5}{*}{$\begin{array}{c}\text { Pilot } \\
\text { Briefing } \\
\text { Pilot } \\
\text { Training }\end{array}$} & $\begin{array}{l}\text { Review } \\
\text { Review } \\
\end{array}$ & \multirow{17}{*}{$\begin{array}{l}\text { Pilot } \\
\text { Travel }\end{array}$} \\
\hline $\begin{array}{l}: 30 \\
: 45 \\
\end{array}$ & & & \multirow[b]{2}{*}{ Run 3} & \\
\hline $\begin{array}{c}10: 00 \mathrm{am} \\
: 15 \\
: 30 \\
: 45\end{array}$ & & & & \\
\hline $\begin{array}{c}11: 00 \mathrm{am} \\
: 15\end{array}$ & & & $\begin{array}{c}\text { Forms \& } \\
\text { Discussion }\end{array}$ & \\
\hline$: 30$ & & & \multirow{3}{*}{$\begin{array}{c}\text { MEAL } \\
\text { BREAK }\end{array}$} & \\
\hline$: 45$ & & \multirow{3}{*}{$\begin{array}{c}\text { MEAL } \\
\text { BREAK }\end{array}$} & & \\
\hline $\begin{array}{c}12: 00 \mathrm{pm} \\
: 15\end{array}$ & & & & \\
\hline $\begin{array}{l}: 30 \\
: 45\end{array}$ & & & \multirow[b]{2}{*}{ Run 4} & \\
\hline $\begin{array}{c}: 43 \\
01: 00 \mathrm{pm} \\
: 15 \\
: 30 \\
: 45\end{array}$ & & \multirow[t]{2}{*}{ Run 1} & & \\
\hline \multirow{4}{*}{$\begin{array}{c}02: 00 \mathrm{pm} \\
: 15 \\
: 30 \\
: 45\end{array}$} & & & \multirow{3}{*}{$\begin{array}{c}\begin{array}{c}\text { Forms \& } \\
\text { Discussion }\end{array} \\
\text { BREAK } \\
\end{array}$} & \\
\hline & & \multirow{3}{*}{$\begin{array}{c}\begin{array}{c}\text { Forms \& } \\
\text { Discussion }\end{array} \\
B R E A K \\
\end{array}$} & & \\
\hline & & & & \\
\hline & & & \multirow[b]{2}{*}{ Debriefing } & \\
\hline $\begin{array}{c}03: 00 \mathrm{pm} \\
: 15 \\
: 30\end{array}$ & & \multirow[t]{3}{*}{ Run 2} & & \\
\hline$: 45$ & & & \multirow{3}{*}{$\begin{array}{l}\text { Buffer } \\
\text { Buffer } \\
\text { Buffer } \\
\text { Buffer } \\
\text { Buffer }\end{array}$} & \\
\hline $\begin{array}{c}04: 00 \mathrm{pm} \\
: 15\end{array}$ & & & & \\
\hline $\begin{array}{l}: 30 \\
: 45\end{array}$ & & $\begin{array}{c}\text { Forms \& } \\
\text { Discussion }\end{array}$ & & \\
\hline
\end{tabular}

E-1 


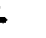

$=$ 


\section{Appendix F \\ Pilot Forms}


* 


\section{Air / Ground Integration Experiment (AGIE) Flight Crew Post-Run Form}

This form is to be completed by pilots participating in AGIE and requests information regarding your overall experiences and judgments about the simulation.

Your name will not be listed or appear in any reports to ensure your anonymity and to encourage unbiased reporting. Findings will be reported generically (Pilot A, B, C, etc.).

When making your ratings, please consider all levels of the scale. You are encouraged to write any additional comments you feel are important. 


\section{Air / Ground Integration Experiment (AGIE) Pilot Post-Run Form}

Date:

Pilot ID\#:

Position: Captain or First Officer (circle one)

Scenario:

Run\#:

Where applicable, please circle the most accurate response.

1. Rate the amount of uncertainty (if any) about who had authority (controller, pilot or both) for maintaining separation during the last set of scenarios.
Very Low
2
3
Moderate
4
5
Very High

2. Rate your physical workload level (e.g., data entry, flying the aircraft, communications, etc.) during this last set of scenarios.

$\begin{array}{ccccc}1 & 2 & 3 & 4 & 5 \\ \text { Very Low } & & \text { Moderate } & & \text { Very High }\end{array}$

Comments.

3. Rate your mental workload level (e.g., planning, predicting, and monitoring, etc.) during this last set of scenarios.

$\begin{array}{ccccc}1 & 2 & 3 & 4 & 5 \\ \text { Very Low } & \text { Moderate } & & \text { Very High }\end{array}$

Comments. 
4. Rate your overall workload level (physical and mental combined) during this last set of scenarios.

$\begin{array}{ccccc}1 & 2 & 3 & 4 & 5 \\ \text { Very Low } & & \text { Moderate } & & \text { Very High }\end{array}$

Comments.

5. Identify events that significantly affected your overall workload during this last set of scenarios.

6. Rate the overall workload associated with coordination and communication between crew members.

$\begin{array}{ccccc}1 & 2 & 3 & 4 & 5 \\ \text { Very Low } & & \text { Moderate } & & \text { Very High }\end{array}$

Comments.

7. Rate the overall workload associated with maintaining air-to-ground communications.
Very Low
2
3
Moderate
4
5

Comments. 
8. Rate the amount of time available for air to ground communications.

Too Little

2

3

Adequate

4

5

Too Much

Comments.

9. Rate the amount of time available for crew coordination and communication.

Too Little

2

3

Adequate

4

5

Too Much

Comments.

10. During the last set of scenarios, I felt rushed:

$\begin{array}{ccc}1 & 2 & 3 \\ \text { Not at All } & & \text { About Half of } \\ \text { the Time }\end{array}$

4 5

the Time

All of the

Time

Comments.

11. During the last set of scenarios, I felt bored:

$\begin{array}{ccc}1 & 2 & 3 \\ \text { Not at All } & \begin{array}{c}\text { About Half of } \\ \text { the Time }\end{array}\end{array}$

4

All of the

Time

Comments. 
The term overall situation awareness refers to what is commonly known as the pilot's

"staying ahead of the aircraft" where the pilot has a thorough understanding of the current situation and can take appropriate action as necessary.

12. Rate your level of overall situation awareness during this run.
Very Low
2
3
4
5
Moderate
Very High

Comments.

13. Please provide any additional comments or concerns you may have about this simulation. 
ID\#:

Run\#

Captain / First Officer

\section{Air / Ground Integration Experiment (AGIE) Flight Crew Post-Run Form}

This form is to be completed by pilots participating in AGIE and requests information regarding your overall experiences and judgments about the simulation.

Your name will not be listed or appear in any reports to ensure your anonymity and to encourage unbiased reporting. Findings will be reported generically (Pilot A, B, C, etc.).

When making your ratings, please consider all levels of the scale. You are encouraged to write any additional comments you feel are important. 
Date:

\section{Air / Ground Integration Experiment (AGIE)} Pilot Post-Run Form

Pilot ID\#:

Position: Captain or First Officer (circle one)

Scenario:

Run\#:

Where applicable, please circle the most accurate response.

1. Rate the amount of uncertainty (if any) about who had authority (controller, pilot or both) for maintaining separation during the last set of scenarios.

$\begin{array}{ccccc}1 & 2 & 3 & 4 & 5 \\ \text { Very Low } & & \text { Moderate } & & \text { Very High }\end{array}$

2. Rate your physical workload level (e.g., data entry, flying the aircraft, communications, etc.) during this last set of scenarios.
1
Very Low
2
3
Moderate
4
5
Very High

Comments.

3. Rate your mental workload level (e.g., planning, predicting, and monitoring, etc.) during this last set of scenarios.
1
2
Very Low
3
Moderate
4
5
Very High

Comments. 
4. Rate your overall workload level (physical and mental combined) during this last set of scenarios.

$$
\begin{aligned}
& 1 \\
& \text { Very Low }
\end{aligned}
$$

2

3

Moderate

4 5

Comments.

5. Identify events that significantly affected your overall workload during this last set of scenarios.

6. Rate the overall workload associated with coordination and communication between crew members
Very Low
2
3
Moderate
4
Very High

Comments.

7. Rate the overall workload associated with maintaining air-to-ground communications.
1
Very Low
2
3
Moderate
4
Very High

Comments. 
8. Rate the amount of time available for air to ground communications.
Too Little
2
3
4
Adequate
Too Much

Comments.

9. Rate the amount of time available for crew coordination and communication.

$\begin{array}{ccccc}1 & 2 & 3 & 4 & 5 \\ \text { Too Little } & & \text { Adequate } & & \text { Too Much }\end{array}$
Comments.

10. How timely was the conflict alert?

$\begin{array}{ccccc}1 & 3 & 4 & 5 \\ \text { Too Early } & 2 & \text { Adequate } & & \text { Too Late } \\ \text { mments. } & & & \end{array}$

\section{Comments.}

11. How easy was it to detect a potential conflict prior to a system alert or a controller traffic advisory?

$\begin{array}{ccccc}1 & 2 & 3 & 4 & 5 \\ \text { Not Easy } & & \text { Moderately Easy } & & \text { Very Easy } \\ \text { Comments. } & & & \end{array}$


12. During the last set of scenarios, I felt rushed:

1 2

Not at All

\section{Comments.}

3

About Half of the Time
4 5

All of the

Time

13. During the last set of scenarios, I felt bored:

1 .

Not at All

2

Comments.
3

About Half of

the Time
4

5

All of the

Time

14. Compared to current operations the procedures used in this set of scenarios resulted in a level of safety that was:

$\begin{array}{ccc}1 & 2 & 3 \\ \text { Compromised } & & \text { Unchanged } \\ \text { If enhanced, why? } & & \end{array}$

4

5

Enhanced

If compromised, why? 
The term overall situation awareness refers to what is commonly known as the pilot's

"staying ahead of the aircraft" where the pilot has a thorough understanding of the current situation and can take appropriate action as necessary.

15. Rate your level of overall situation awareness during this run.

$\begin{array}{ccccc}1 & 3 & 4 & 5 \\ \text { Very Low } & 2 & \text { Moderate } & & \text { Very High }\end{array}$

16. Please estimate the percentage of time you spent monitoring the CDTI: $\%$ Did monitoring the CDTI interfere with your other flight duties? Yes No

17. Please provide any additional comments or concerns you may have about this simulation. 
ID\#:

Run\#

Captain / First Officer

\section{Air / Ground Integration Experiment (AGIE) Flight Crew Post-Run Form}

This form is to be completed by pilots participating in AGIE and requests information regarding your overall experiences and judgments about the simulation.

Your name will not be listed or appear in any reports to ensure your anonymity and to encourage unbiased reporting. Findings will be reported generically (Pilot A, B, C, etc.).

When making your ratings, please consider all levels of the scale. You are encouraged to write any additional comments you feel are important. 


\section{Air / Ground Integration Experiment (AGIE)}

\section{Date:}

\section{Pilot Post-Run Form}

Pilot ID\#:

Position: Captain or First Officer (circle one)

Scenario:

Run\#:

Where applicable, please circle the most accurate response.

1. Rate the amount of uncertainty (if any) about who had authority (controller, pilot or both) for maintaining separation during the last set of scenarios.
Very Low
2
3
Moderate
4
5
Very High

2. Rate your physical workload level (e.g., data entry, flying the aircraft, communications, etc.) during this last set of scenarios.

$\begin{array}{ccccc}1 & 2 & 3 & 4 & 5 \\ \text { Very Low } & 2 & & \text { Very High } \\ \text { Comments. } & & \text { Moderate } & & \end{array}$

3. Rate your mental workload level (e.g., planning, predicting, and monitoring, etc.) during this last set of scenarios.

$\begin{array}{ccccc}1 & 2 & 3 & 4 & 5 \\ \text { Very Low } & & \text { Moderate } & & \text { Very High } \\ \text { Comments. } & & & & \end{array}$


4. Rate your overall workload level (physical and mental combined) during this last set of scenarios.

1

Very Low

Comments.
2

Moderate
4

5

Very High

5. Identify events that significantly affected your overall workload during this last set of scenarios.

6. Rate the overall workload associated with maintaining aircraft separation. (e.g., monitoring and planning).
Very Low
2
3
Moderate
4
5
Very High

Comments.

7. Rate the overall workload associated with maintaining air-to-air communications.

$\begin{array}{ccccc}1 & 2 & 3 & 4 & 5 \\ \text { Very Low } & \text { Moderate } & & \text { Very High }\end{array}$

Comments. 
8. Rate the overall workload associated with coordination and communication between crew members.

$\begin{array}{ccccc}1 & 2 & 3 & 4 & 5 \\ \text { Very Low } & & \text { Moderate } & & \text { Very High }\end{array}$

Comments.

9. Rate the overall workload associated with maintaining air-to-ground communications.

$\begin{array}{ccccc}1 & 2 & 3 & 4 & 5 \\ \text { Very Low } & \text { Moderate } & & \text { Very High }\end{array}$

Comments.

10. Rate the amount of time available to detect, monitor and resolve conflicts and maintain self-separation with other aircraft.

12

Too Little

Comments.
Adequate

4

Too Much

11. Rate the amount of time available for air to air communications.

Too Little

2 Tomments.
4

Adequate
5

Too Much 
12. Rate the amount of time available for air to ground communications.

1

Too Little

Comments.
2

Adequate
4

5

Too Much

13. Rate the amount of time available for crew coordination and communication.

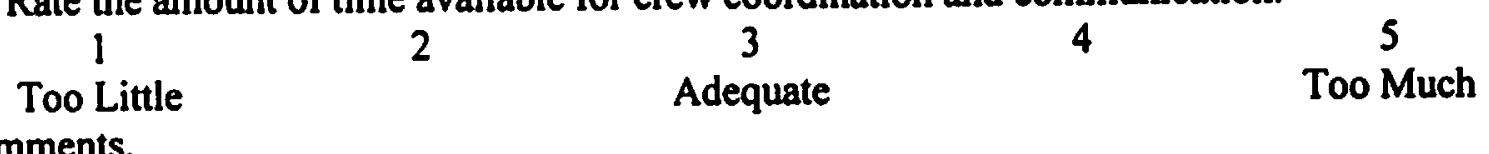

14. How timely was the conflict alert?

1

Too Early

3

Adequate

4

5

Too Late

Comments.

15. How easy was it to detect a potential conflict prior to a system alert or a controller traffic advisory?
1
Not Easy
2
3
Moderately Easy
4
Very Easy
Comments. 
16. Please rate the effectiveness of the alerting logic and display for conducting free flight self-separation operations.

$\begin{array}{ccccc}1 & 2 & 3 & 4 & 5 \\ \text { Not } & 2 & \text { Moderately } & \text { Very } \\ \text { Effective } & & \text { Effective } & & \text { Effective }\end{array}$

Comments.

17. Identify factors that interfered with maintaining separation during this last set of scenarios.

18. During the last set of scenarios, I felt rushed:

1

Not at All

Comments.
2

3

About Half of

the Time
4

All of the

Time

19. During the last set of scenarios, I felt bored:

1
Not at All $\quad \begin{gathered}3 \\ \text { About Half of } \\ \text { the Time }\end{gathered}$

4

All of the

Time

Comments. 
20. Compared to current operations the procedures used in this last set of scenarios resulted in a level of safety that was:
1
2
3
4
5
Unchanged
Enhanced

Compromised

If enhanced, why?.

If compromised, why?

The term overall situation awareness refers to what is commonly known as the pilot's "staying ahead of the aircraft" where the pilot has a thorough understanding of the current situation and can take appropriate action as necessary.

21. Rate your level of overall situation awareness during this run.
1
Very Low
2
3
Moderate
4
Very High

Comments.

22. Rate how comfortable you were with sharing separation responsibility with the controller.

$\begin{array}{ccc}1 & 2 & 3 \\ \text { Not } & & \text { Moderately } \\ \text { Comfortable } & & \text { Comfortable }\end{array}$

4

Comments. 
23. Rate how often you monitored other air-air communications.

1 Never

Comments.
2

3 Sometimes
4 5

Always

24. Rate the usefulness of monitoring other air-air communications.

$\begin{array}{ccccc}1 & 2 & 3 & 4 & 5 \\ \text { Not Useful } & \begin{array}{c}\text { Moderately } \\ \text { Useful }\end{array} & \text { Very Useful }\end{array}$

Comments.

25. Rate the impact of the shared-separation operations concept for performing your job.

$\begin{array}{ccccc}1 & 2 & 3 & 4 & 5 \\ \text { Detrimental } & - & \text { No Impact } & & \text { Helpful }\end{array}$
Comments.

26. Please estimate the percentage of time you spent monitoring the CDTI: $\%$ Did monitoring the CDTI interfere with your other flight duties? Yes No 
27. If you cancelled Free Flight, identify the reasons.

28. Rate the usefulness of traffic advisories that were issued by the controller.

$\begin{array}{ccccc}1 & 2 & 3 & 5 \\ \text { Not Useful } & 2 & \begin{array}{c}\text { Moderately } \\ \text { Useful }\end{array} & & \text { Very Useful }\end{array}$

29. Please provide any additional comments or concerns you may have about this simulation. 
ID\#:

Run\#

Captain / First Officer

\section{Air / Ground Integration Experiment (AGIE) Flight Crew Post-Run Form}

This form is to be completed by pilots participating in AGIE and requests information regarding your overall experiences and judgments about the simulation.

Your name will not be listed or appear in any reports to ensure your anonymity and to encourage unbiased reporting. Findings will be reported generically (Pilot A, B, C, etc.).

When making your ratings, please consider all levels of the scale. You are encouraged to write any additional comments you feel are important. 


\section{Air / Ground Integration Experiment (AGIE) Pilot Post-Run Form}

Date: 1

Pilot ID\#:

Position: Captain or First Officer (circle one)

Scenario:

Run\#:

Where applicable, please circle the most accurate response.

1. Rate the amount of uncertainty (if any) about who had authority (controller, pilot or both) for maintaining separation during the last set of scenarios.
Very Low
2
3
4
Very High

2. Rate your physical workload level (e.g., data entry, flying the aircraft, communications, etc.) during this last set of scenarios.
1
Very Low
2
3
4
Moderate
Very High

Comments.

3. Rate your mental workload level (e.g., planning, predicting, and monitoring, etc.) during this last set of scenarios.

$\begin{array}{ccc}1 & 2 & 3 \\ \text { Very Low } & & \text { Moderate } \\ \text { mments. } & & \end{array}$

4

Comments. 
4. Rate your overall workload level (physical and mental combined) during this last set of scenarios.

$\begin{array}{ccccc}1 & 2 & 3 & 4 & 5 \\ \text { Very Low } & \text { Moderate } & & \text { Very High } \\ \text { Comments. } & & & & \end{array}$

5. Identify events that significantly affected your overall workload during this last set of scenarios.

6. Rate the overall workload associated with maintaining aircraft separation. (e.g., monitoring and planning).

$\begin{array}{ccccc}1 & 2 & 3 & 4 & 5 \\ \text { Very Low } & \text { Moderate } & & \text { Very High } \\ \text { Comments. } & & & \end{array}$

7. Rate the overall workload associated with maintaining air-to-air communications.
1
Very Low
2
3
Moderate
4
5

Comments. 
8. Rate the overall workload associated with coordination and communication between crew members.
1
2
3
4
5
Very Low
Moderate
Very High

Comments.

9. Rate the overall workload associated with maintaining air-to-ground communications.

123

Very Low

Comments.

Moderate

4

5

Very High

10. Rate the amount of time available to detect, monitor and resolve conflicts and maintain self-separation with other aircraft.
Too Little
2
3
Adequate
4

Comments.

11. Rate the amount of time available for air to air communications.
1
Too Little
2
3
4
5
Adequate
Too Much

Comments. 
12. Rate the amount of time available for air to ground communications.
1
Too Little
2
3
4
Adequate
Too Much

Comments.

13. Rate the amount of time available for crew coordination and communication.

$\begin{array}{ccccc}1 & 2 & 3 & 4 & 5 \\ \text { Too Little } & \text { Adequate } & & \text { Too Much }\end{array}$
Comments.

14. How timely was the conflict alert?

$\begin{array}{ccccc}1 & 2 & 3 & 4 & 5 \\ \text { Too Early } & 2 & \text { Adequate } & & \text { Too Late }\end{array}$

Comments.

15. How easy was it to detect a potential conflict prior to a system alert or a controller traffic advisory?

$\begin{array}{ccccc}1 & 2 & 3 & 4 & 5 \\ \text { Not Easy } & & \text { Moderately Easy } & & \text { Very Easy } \\ \text { Comments. } & & & \end{array}$


16. Please rate the effectiveness of the alerting logic and display for conducting free flight self-separation operations.

$\begin{array}{ccccc}1 & 3 & 4 & 5 \\ \text { Not } & 2 & \begin{array}{c}\text { Moderately } \\ \text { Effective }\end{array} & & \begin{array}{c}\text { Very } \\ \text { Effective }\end{array}\end{array}$

Comments.

17. Identify factors that interfered with maintaining separation during this last set of scenarios.

18. During the last set of scenarios, I felt rushed:

$\begin{array}{cc}1 & 3 \\ \text { Not at All } & \text { About Half of } \\ \text { the Time }\end{array}$

4 5

All of the

Time

\section{Comments.}

19. During the last set of scenarios, 1 felt bored:

$\begin{array}{cc}1 & 3 \\ \text { Not at All } & 2 \\ \text { About Half of } \\ \text { the Time }\end{array}$

4

5

All of the

Time

\section{Comments.}


20. Compared to current operations the procedures used in this set of scenarios resulted in a level of safety that was:

1

Compromised

If enhanced, why?
2

3

Unchanged
4 5

Enhanced

If compromised, why?

The term overall situation awareness refers to what is commonly known as the pilot's "staying ahead of the aircraft" where the pilot has a thorough understanding of the current situation and can take appropriate action as necessary.

21. Rate your level of overall situation awareness during this run.
Very Low
2
3
4
Moderate
Very High

Comments.

22. Rate how comfortable you were with sharing separation responsibility with the controller.

1

Not

Comfortable

Comments.
2

$\stackrel{3}{\text { Moderately }}$ Comfortable

4

5 Very

Comfortable 
23. Rate how often you monitored other air-air communications.

1 Never 2 3 Sometimes

4 5

Always Comments.

24. Rate the usefulness of monitoring other air-air communications.
Not Useful
2
3
Moderately
4
Useful
$\stackrel{5}{\text { Very Useful }}$

Comments.

25. Rate the impact of the shared-separation operations concept for performing your job.
Detrimental
2
3
No Impact
4
Helpful

\section{Comments.}

26. Please estimate the percentage of time you spent monitoring the CDTI: $\%$ Did monitoring the CDTI interfere with your other flight duties? Yes No 
27. If you cancelled Free Flight, identify the reasons.

28. Rate the usefulness of traffic advisories that were issued by the controller.

$\begin{array}{ccccc}1 & 2 & 3 & 4 \\ \text { Not Useful } & 2 & \text { Useful } & \text { Very Useful }\end{array}$

29. Please provide any additional comments or concerns you may have about this simulation. 


\section{Air / Ground Integration Experiment (AGIE) \\ Pilot Exit Form}

Date:

Captain or First Officer (circle one)

ID\#:

Total Flying Hours:

Total Hours on B747-400:

Where applicable, please circle the most accurate response.

1. Rate the adequacy of the simulation briefing.

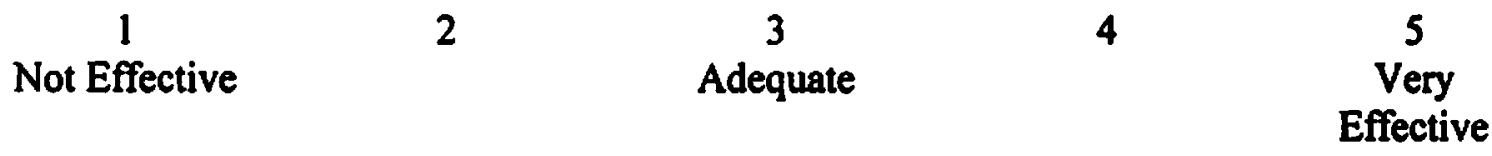

Comments.

2. Rate the adequacy of the simulation training.

$\begin{array}{ccccc}1 & 2 & 3 & 4 & 5 \\ \text { Not Effective } & 2 & \text { Adequate } & & \begin{array}{c}\text { Very } \\ \text { Effective }\end{array}\end{array}$

Comments.

3. Please rate the effectiveness of the CDTI and alerting system for safe flight operations. Not Effective 2 3 Moderately

Effective

Comments.

Effective 
4. Rate the amount of the information on the CDTI available to identify and resolve conflicts.
1
Too Little
2
3
Adequate
4
Too Much

\section{Comments.}

5. How much time did the alerting system provide for strategic self-separation?

Too Little

2

3

Adequate

4

Too Much

\section{Comments.}

6. What additional information is needed, if any?

7. What information was not useful or added clutter, if any? 
8. What strategies did you use to de-clutter the display?

9. Identify your strategies for self-separation.

10. During any of the runs were you ever unsure about which of the authority conditions (e.g. Shared authority) you were flying under? Please rate your level of uncertainty.

$\begin{array}{ccccc}1 & 2 & 3 & 4 & 5 \\ \text { Never } & & \text { Occasionally } & & \text { Frequently } \\ \text { Uncertain } & & \text { Uncertain } & & \text { Uncertain }\end{array}$

Comments. 
11. In your opinion how much vertical and lateral separation represents an effective safety margin? Please specify in terms of nautical miles and feet.

vertical (ft.) horizontal (nm)

\section{Comments.}

12. What additional pilot training or skills (if any) do you think might be necessary in a shared and flight deck authority environment compared with an ATC authority environment?

13. What problems (if any) do you anticipate in negotiating separation maneuvers with other aircraft in conflict with you?

14. What procedures would you suggest to facilitate the implementation of sharedseparation operations? 
15. Rate the usefulness of the right-of-way rules in negotiation and initiating contact with a conflicting aircraft?

$\begin{array}{ccccc}1 & 2 & 3 & 5 & 5 \\ \text { Not Useful } & & \begin{array}{c}\text { Useful } \\ \text { Usely }\end{array} & & \text { Very Useful }\end{array}$

Comments.

The term overall situation awareness refers to what is commonly known as the pilot's

"staying ahead of the aircraft" where the pilot has a thorough understanding of the current situation and can take appropriate action as necessary.

16. Rate the usefulness of the air to air frequency maintaining situation awareness (see definition above)?

$\begin{array}{cccc}1 & 2 & 3 & 5 \\ \text { Not Useful } & 2 & 4 \\ \text { Useful } & & \text { Very Useful }\end{array}$

Comments.

17. How distracting was the conflict detection button (EVENT RCD) to your overall task?

1

Not At All

Distracting
2

3
Somewhat
Distracting

4 5

Very Distracting

Comments. 
18. How representative do you think your use of the conflict detection button (EVENT $\mathrm{RCD})$ reflects the true timing of when you detected a conflict?

1

Not

Representative
2
3

Somewhat

Representative
4

5

Very

Representative

Comments.

19. What can be done to improve simulation fidelity? If we were to conduct future shared-separation related research, what improvements in scenario, traffic, phraseology, and simulation would you suggest?

20. What additional information and decision support tools do you recommend under the following flight authority conditions:

Current

Operations

Controller

Authority

Shared

Authority

Flight Deck

Authority 
21. Did any of the conflict scenarios appear similar? Yes

No

If so, in regards to which of the following parameters: conflict angle conflict timing ownship flight plan intruders flight plan general location of intruder traffic pattern other 


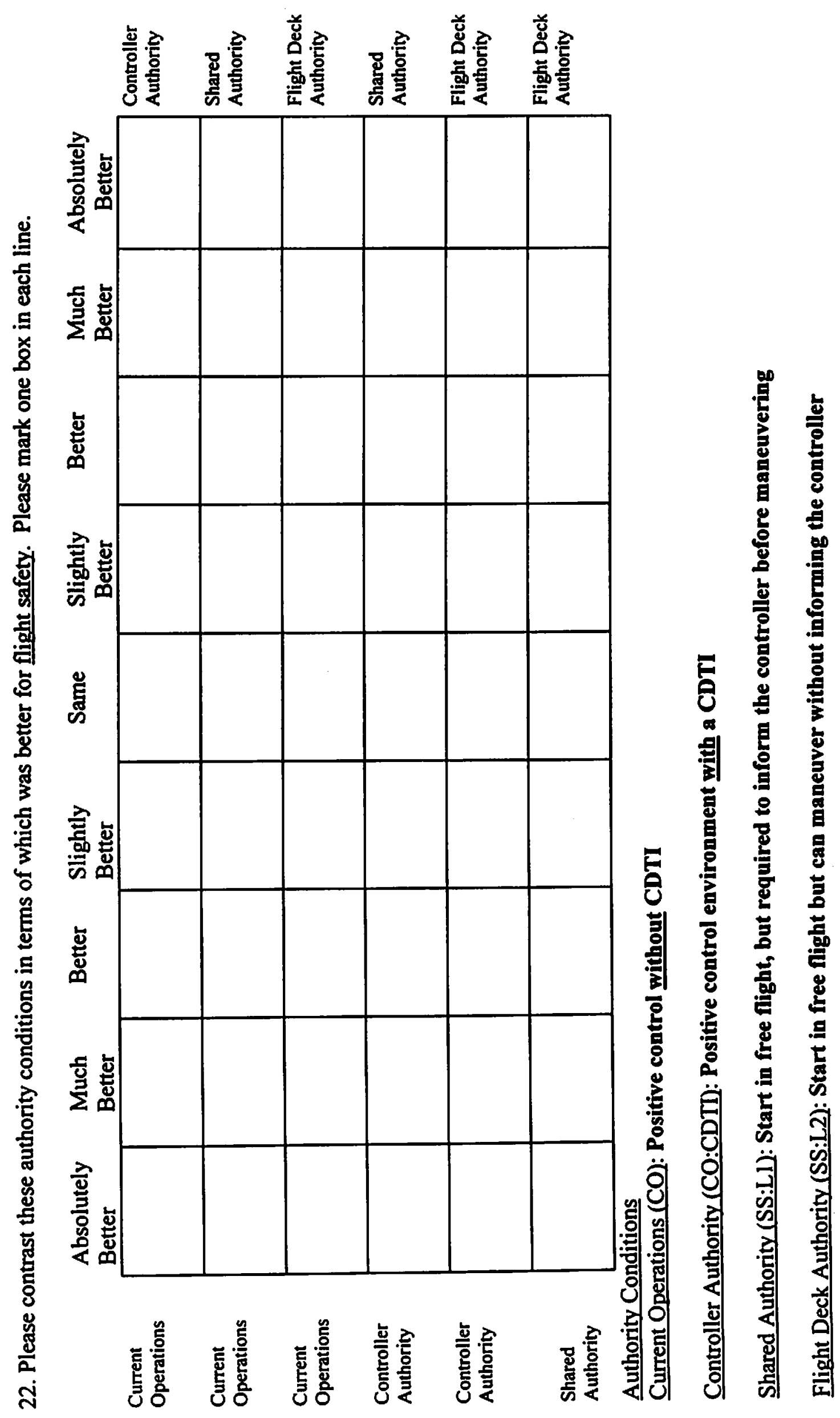




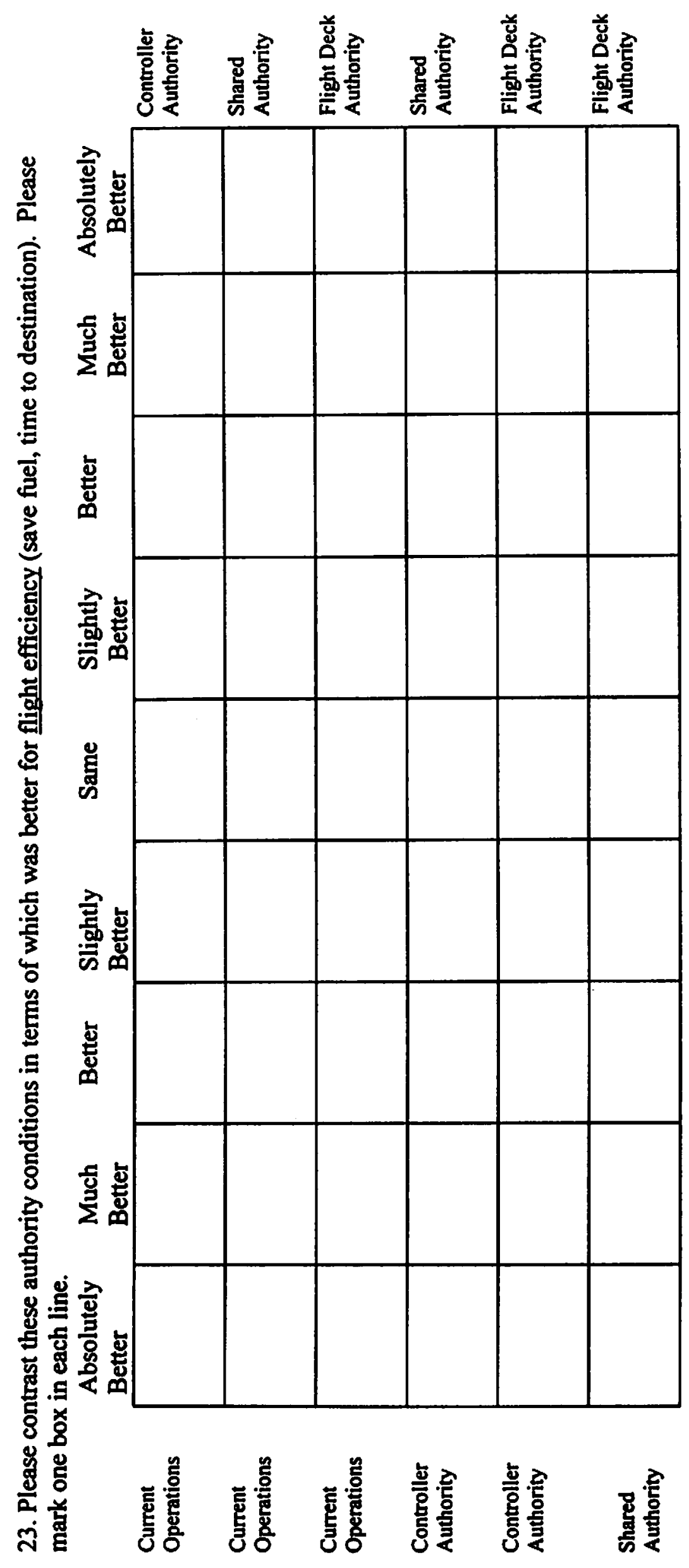

$\infty$ 


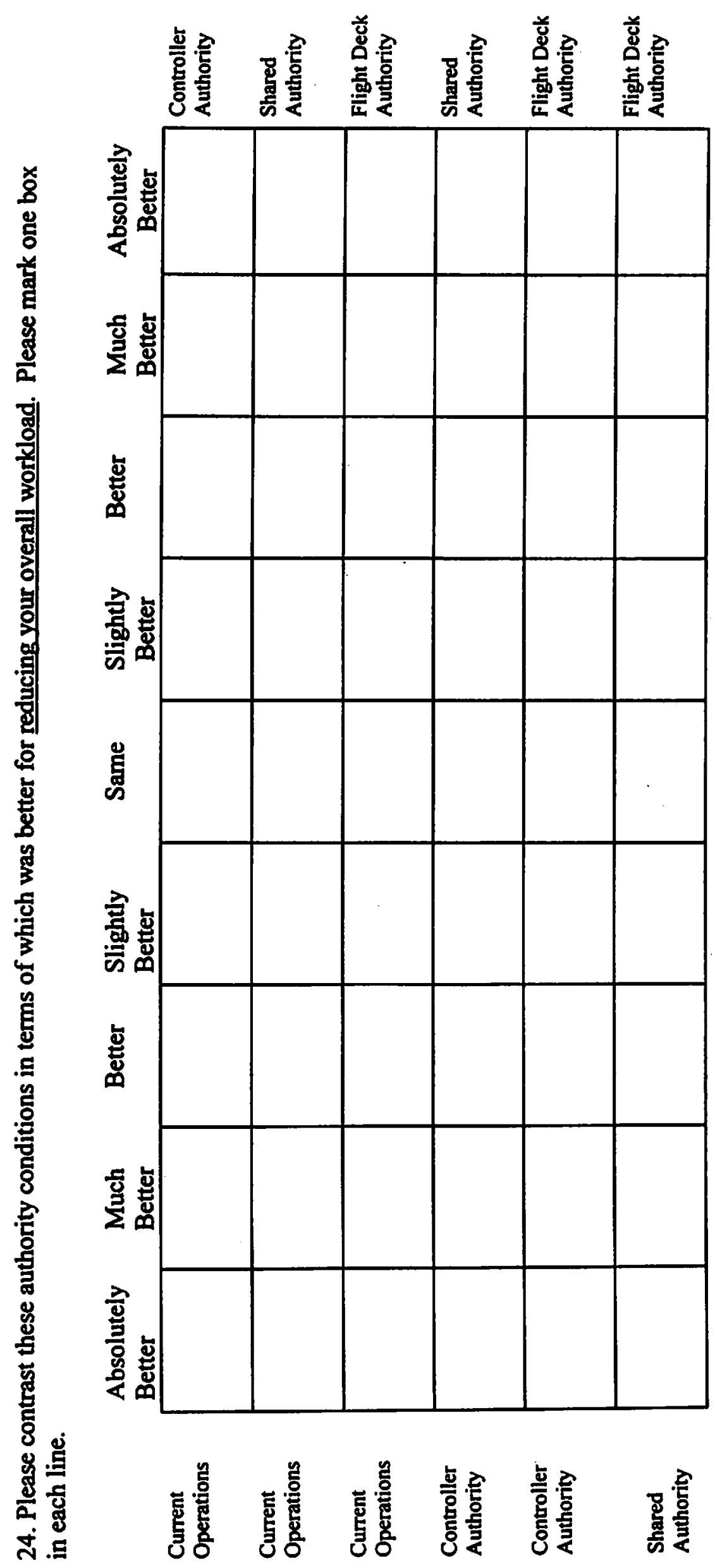

号 


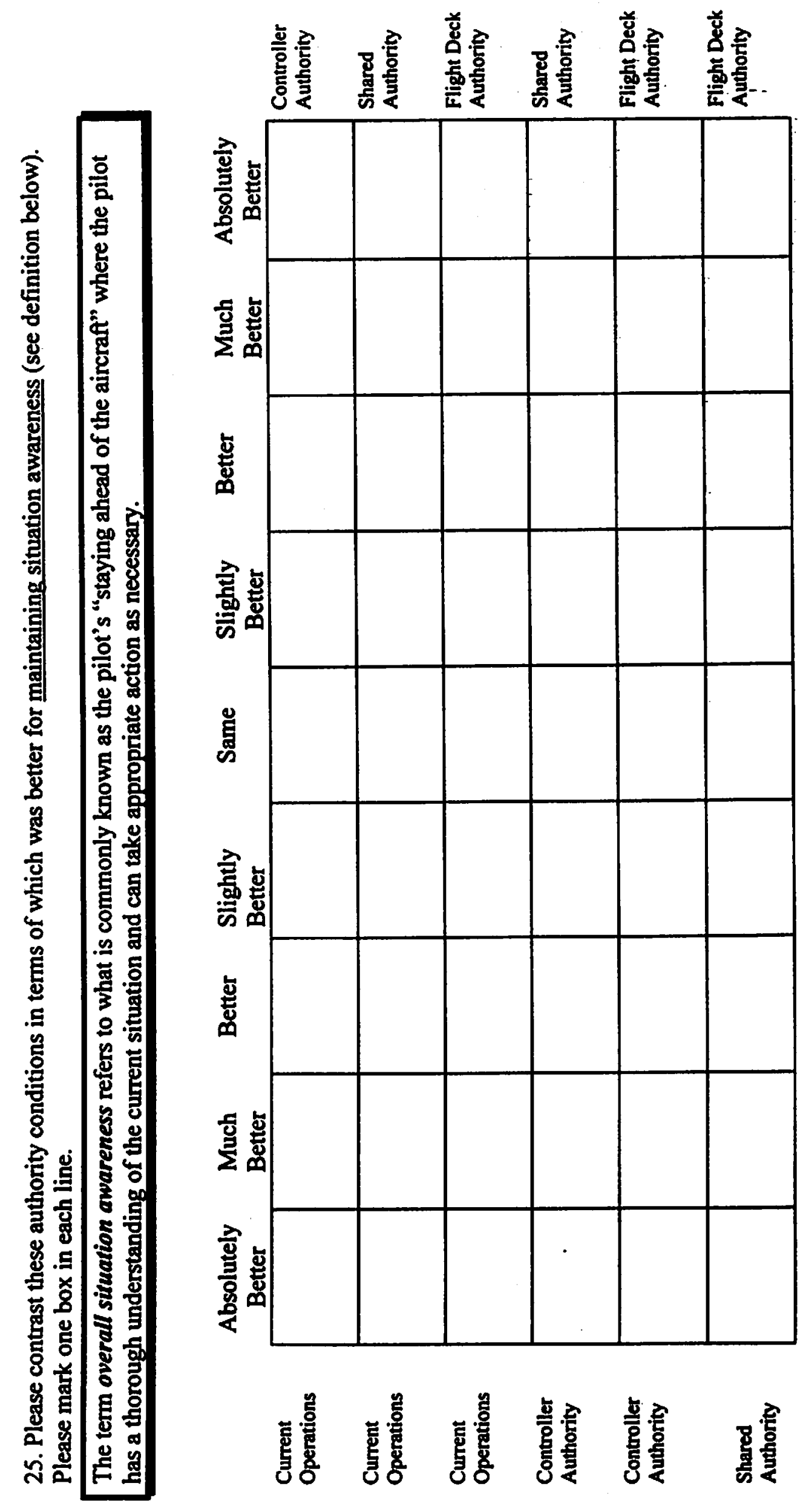

I 
Appendix G

ANOVA Results 


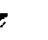


Section G-1. Controller Ratings for the Amount of Time Available to Assure Safe Aircraft Separation and Complete Required Coordination

The researchers performed two-way ANOVAs with the factors of Control Condition (CO, CO:CDTI, SS:L1, SS:L2) and Position (R-Side, D-Side) on controller ratings for the amount of time available to assure safe aircraft separation and complete required coordination. The ANOVAs indicated that the main effect of Control Condition was statistically significant for the amount of time available to assure safe aircraft separation $[F(3,27)=3.75, p<.05]$. No other main effects or interactions were significant for these two ratings. The differences between the four Control Condition means for the amount of time available to assure safe aircraft separation were examined using Tukey HSD posthoc comparisons. The results indicated that significantly more time was available for CO:CDTI compared to SS:L2, but CO:CDTI was not statistically different from CO or SS:L1. There were no reliable differences between CO, SS:L1, and SS:L2.

\section{Section G-2. Controller Ratings for the Level of Safety for Procedures Compared to}

\section{Current Operations}

The researchers performed a two-way ANOVA with the factors of Control Condition (CO, CO:CDTI, SS:L1, SS:L2) and Position (R-Side, D-Side) on controller ratings for the level of safety for procedures compared to current operations. The ANOVA indicated that the main effect of Control Condition was statistically significant $[F(3,27)=27.23$, $p<.01]$. No other main effects or interactions were significant for these ratings. The differences between the four Control Condition means were examined using Tukey HSD post-hoc comparisons. The results indicated that the level of safety was significantly higher for CO and CO:CDTI compared to SS:L1 and SS:L2. There was no reliable difference between CO and CO:CDTI and no difference between SS:L1 and SS:L2.

\section{Section G-3A. Frequency of URET Red and Yellow Alerts}

The researchers performed one-way ANOVAs with only Control Condition (CO, CO:CDTl, SS:L1, SS:L2) on the frequency of URET red and yellow alerts (for each RSide/D-Side controller team). The ANOVAs indicated that there were no statistically significant effects for the frequency of these two alerts. 
Section G-3B. Duration per Alert of URET Red and Yellow Alerts

The researchers performed one-way ANOVAs with only Control Condition (CO, CO:CDTI, SS:L1, SS:L2) on the duration per alert for URET red and yellow alerts (for each R-Side/D-Side controller team). The ANOVAs indicated that the effect of Control Condition was statistically significant for both the duration of red alerts $[F(3,15)=$ $15.73, p<.01]$, and yellow alerts $[F(3,15)=12.96, p<.01]$. The differences between the four Control Condition means were examined using Tukey HSD post-hoc comparisons. The results indicated that red alert durations were longer in SS:L2 compared to CO, CO:CDTI, and SS:L1. There were no reliable differences between $\mathrm{CO}$, CO:CDTI, and SS:L1. Yellow alert durations were longer in SS:L2 compared to CO and SS:L1, but SS:L2 and CO:CDTI were not statistically different. Also, yellow alert durations were longer in CO:CDTI compared to SS:Ll, but CO:CDTI and CO were not statistically different. Lastly, there was no reliable difference between CO and SS:L1.

\section{Section G-4. Minimum Separation Distance (MSD) and Free Fliaht Cancellations} for Planned Conflicts Involving WJHTC Simulation Pilot

The researchers performed one-way ANOVAs with only Control Condition (CO, CO:CDTI, SS:L1, SS:L2) on the MSDs for altitude-resolved and vector-resolved conflicts (for each week of two R-Side/D-Side controller teams). The ANOVAs indicated that the effect of Control Condition was statistically significant for the MSDs of altitude-resolved conflicts $[F(3,3)=27.40, p<.05]$, but not for the MSDs of vectorresolved conflicts. The differences between the four Control Condition means for the MSDs of altitude-resolved conflicts were examined using Tukey HSD post-hoc comparisons. The results indicated that the MSDs were greater in CO and CO:CDTI compared to SS:L1 and SS:L2. There was no reliable difference between $C O$ and CO:CDTI and no difference between SS:L1 and SS:L2.

Section G-5. Controller Ratings for the Amount of Information Available to Resolve Conflicts

The researchers performed a two-way ANOVA with the factors of Control Condition (CO, CO:CDTI, SS:L1, SS:L2) and Position (R-Side, D-Side) on controller ratings for the amount of information available to resolve conflicts. The ANOVA indicated that there were no statistically significant main effects or interactions for these ratings.

\section{Section G-6. Controller Ratings for the URET Conflict Alert Timeliness of the Conflict Probes}

The researchers performed a two-way ANOVA with the factors of Control Condition (CO, CO:CDTI, SS:L1, SS:L2) and Position (R-Side, D-Side) on controller ratings for the timing of the conflict probes. The ANOVA indicated that there were no statistically significant main effects or interactions for these ratings. 
Section G-7. Controller Ratings for the Usefulness of Monitoring Air-to-Air Communications

The researchers performed a two-way ANOVA with the factors of Control Condition (SS:L1, SS:L2) and Position (R-Side, D-Side) on controller ratings for the usefulness of monitoring air-to-air communications. Only the ratings from controllers who monitored air-to-air communications were considered for this analysis. The ANOVA indicated that there were no statistically significant main effects or interactions for these ratings.

\section{Section G-8. Controller Ratings for the Helpfulness of the Shared-Separation Concept}

The researchers performed a two-way ANOVA with the factors of Control Condition (SS:L1, SS:L2) and Position (R-Side, D-Side) on controller ratings for the helpfulness of the shared-separation concept. The ANOVA indicated that there were no statistically significant main effects or interactions for these ratings.

\section{Section G-9. Frequency of URET Trial Plans}

The researchers performed a one-way ANOVA with only Control Condition (CO, CO:CDTI, SS:L1, SS:L2) on the frequency of URET trial plans (for each week of two RSide/D-Side controller teams). The ANOVA indicated that the effect of Control Condition was statistically significant $[F(3,6)=10.54, p<.01]$. The differences between the four Control Condition means were examined using Tukey HSD post-hoc comparisons. The results indicated that significantly more trial plans were formed for $\mathrm{CO}$ and CO:CDTI compared to SS:L2. There were no reliable differences between CO, CO:CDTI, and SS:Ll and no differences between SS:L1 and SS:L2.

\section{Section G-10. Controller Ratings for Physical, Mental, and Overall Workload}

The researchers performed two-way ANOVAs with the factors of Control Condition (CO, CO:CDTI, SS:L1, SS:L2) and Position (R-Side, D-Side) on controller ratings for physical, mental, and overall workload. The ANOVAs indicated that the main effect of Control Condition was statistically significant for both mental workload $[F(3,30)=6.59$, $p<.01]$, and overall workload $[F(3,30)=5.27, p<.01]$. No other main effects or interactions were significant for these three workload ratings. The differences between the four Control Condition means for both mental and overall workload were examined using Tukey HSD post-hoc comparisons. The results indicated that mental workload was significantly higher for SS:L1 compared to CO and SS:L2, but SS:L1 and CO:CDTI were not statistically different. There were no reliable differences between CO, CO:CDTI, and SS:L2. The results were similar for overall workload. Overall workload was significantly higher for SS:L1 compared to CO and SS:L2, but SS:L1 and CO:CDTI were not statistically different. There were no reliable differences between CO, CO:CDTI, and SS:L2. 
Section G-11. Controller Workload Ratings for Maintaining Aircraft Separation, Land Line Coordination, R Side-to-D Side Coordination, Ground-to-Air Transmissions, and URET Coordination

The researchers performed two-way ANOVAs with the factors of Control Condition (CO, CO:CDTI, SS:L1, SS:L2) and Position (R-Side, D-Side) on workload ratings for maintaining aircraft separation, radar-to-data coordination, and URET coordination. The researchers performed one-way ANOVAs with only Control Condition (CO, CO:CDTI, SS:L1, SS:L2) on workload ratings for ground-to-ground coordination (for each D-Side controller) and ground-to-air communication (for each R-Side controller). The ANOVAs indicated that there were no statistically significant main effects or interactions for these five workload ratings.

\section{Section G-12. Controller Ratings for Feeling Rushed and Bored}

The researchers performed two-way ANOVAs with the factors of Control Condition (CO, CO:CDTI, SS:L1, SS:L2) and Position (R-Side, D-Side) on controller ratings for feeling rushed and bored during the simulation. The ANOVAs indicated that there were no statistically significant main effects or interactions for these two ratings.

\section{Section G-13. Controller Ratings for Overall Situation Awareness}

The researchers performed a two-way ANOVA with the factors of Control Condition (CO, CO:CDTI, SS:L1, SS:L2) and Position (R-Side, D-Side) on controller ratings for their level of overall situation awareness. The ANOVA indicated that there were no statistically significant main effects or interactions for these ratings.

\section{Section G-14. Controller Interval Workload Ratings}

The researchers performed a two-way ANOVA with the factors of Control Condition (CO, CO:CDTI, SS:L1, SS:L2) and Position (R-Side, D-Side) on the mean workload ratings across the intervals. The ANOVA indicated that there were no statistically significant main effects or interactions for these ratings.

\section{Section G-15. Expert Observer Ratings of Controller Physical Taskload}

The researchers performed a two-way ANOVA with the factors of Control Condition (CO, CO:CDTI, SS:L1, SS:L2) and Position (R-Side, D-Side) on observer ratings of controller physical taskload. The ANOVA indicated that the main effect of Control Condition was statistically significant $[F(3,30)=12.87, p<.01]$. No other main effects or interactions were significant for these ratings. The differences between the four Control Condition means were examined using Tukey HSD post-hoc comparisons. The results indicated that observer ratings of controller physical taskload were significantly higher for SS:L1 compared to CO, CO:CDTI, and SS:L2. There were no reliable differences between CO, CO:CDTI, and SS:L2. 


\section{Section G-16A. Controller Ground-to-Air and Land Line PTTs}

The researchers performed one-way ANOVAs with only Control Condition (CO, CO:CDTI, SS:L1, SS:L2) on the frequency of ground-to-air transmissions (for each RSide controller) and land line transmissions (for each D-Side controller). The ANOVAs indicated that the effect of Control Condition was statistically significant for both the frequency of ground-to-air transmissions $[F(3,9)=8.13, p<.01]$, and land line transmissions $[F(3,9)=6.27, p<.05]$. The differences between the four Control Condition means were examined using Tukey HSD post-hoc comparisons. The results indicated that the frequency of ground-to-air transmissions was significantly lower for SS:L2 compared to CO, CO:CDTI, and SS:L1. There were no reliable differences between CO, CO:CDTI, and SS:L1. The results were similar for land line transmissions. The frequency of land line transmissions was significantly lower for SS:L2 compared to $\mathrm{CO}$ and SS:L1, but SS:L2 and CO:CDTI were not statistically different. There were no reliable differences between $\mathrm{CO}, \mathrm{CO}: \mathrm{CDTI}$, and SS:L1.

\section{Section G-16B. Duration per Transmission of Ground-to-Air and Land Line PTTs}

The researchers performed one-way ANOVAs with only Control Condition (CO, CO:CDTI, SS:L1, SS:L2) on the duration per transmission of ground-to-air transmissions (for each R-Side controller) and land line transmissions (for each D-Side controller). The ANOVAs indicated that there were no statistically significant main effects or interactions for these two ratings. 

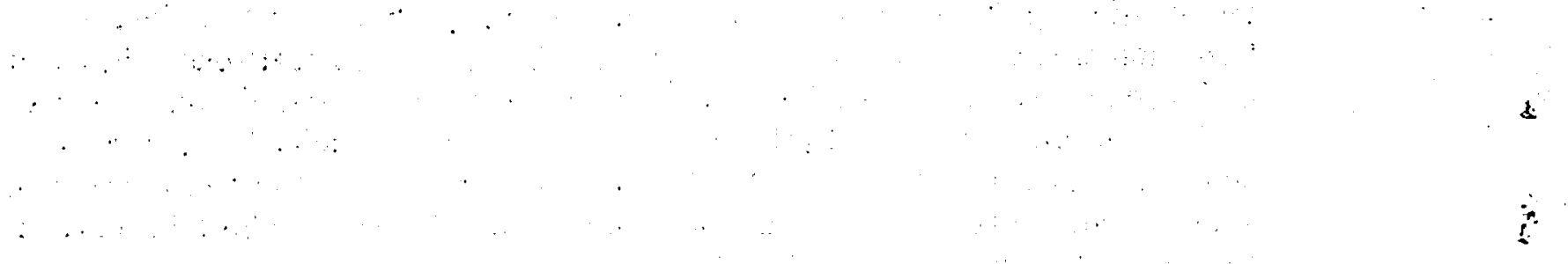

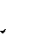


$\therefore-\therefore$

,

$\dot{-}$

$\because$

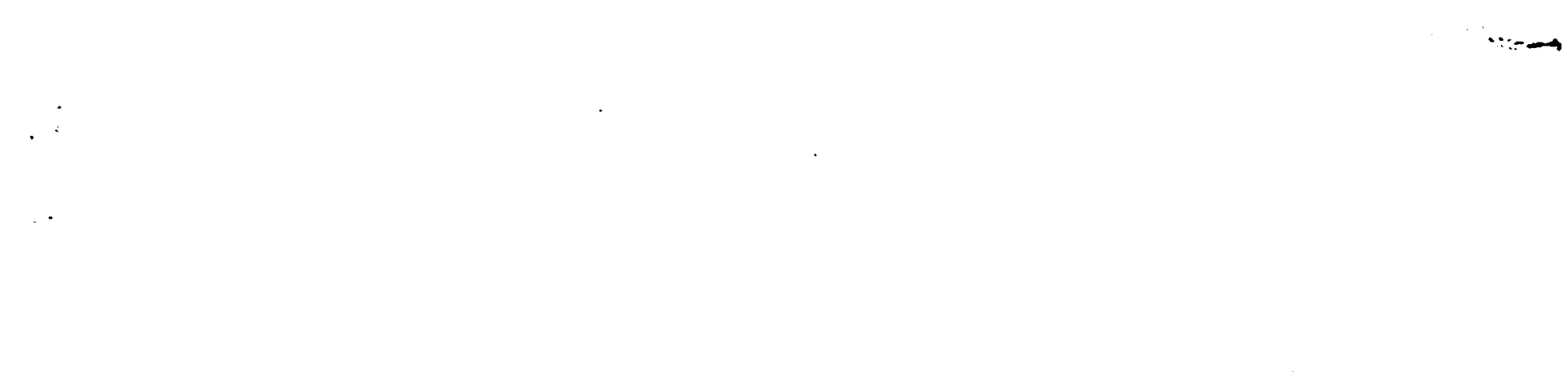

